\title{
Highly E-selective, Stereoconvergent Nickel-Catalyzed Suzuki-Miyaura Cross-Coupling of Alkenyl Ethers
}

\author{
Guo-Ming Ho, Heiko Sommer, and Ilan Marek* \\ Schulich Faculty of Chemistry, Technion-Israel Institute of Technology, Technion \\ City, Haifa, 3200009 (Israel) \\ Email: chilanm@technion.ac.il
}

\section{Supporting Information}

\section{Table of contents}

1. General information

2. Preparation of neopentyl arylboronic esters

3. Preparation of alkenyl methyl ethers

4. Optimization of nickel-catalyzed Suzuki-Miyaura cross-coupling of alkenyl ether 1a

5. Nickel-catalyzed Suzuki-Miyaura cross-coupling of alkenyl ethers with different neopentyl boronic esters

6. Scale-up experiment

7. Control experiments to identify isomerization-active species

8. References

9. NMR spectra 


\section{General information}

Unless stated otherwise, chemically based reactions were conducted in flame-dried glassware under a positive pressure of argon. Thin-layer chromatography (TLC) was conducted with E. Merck silica gel 60 F254 pre-coated plates, $(0.25 \mathrm{~mm})$ and visualized by exposure to UV light ( $254 \mathrm{~nm}$ ) or stained with phosphomolybdic acid, or potassium permanganate. Column chromatography was performed using Fluka silica gel $60 \AA$ (40-63mm, 230-400 mesh). NMR spectra were recorded on Bruker spectrometers (AVIII400) and are reported relative to residual deuterated solvent signals. Chemical shifts are reported in parts per million (ppm) with respect to the residual solvent signal $\mathrm{CDCl}_{3}\left({ }^{1} \mathrm{H}\right.$ NMR: $\delta=7.24 ;{ }^{13} \mathrm{C}$ NMR: $\left.\delta=77.00\right)$ or $\mathrm{C}_{6} \mathrm{D}_{6}\left({ }^{1} \mathrm{H}\right.$ NMR: $\delta=7.15 ;{ }^{13} \mathrm{C}$ NMR: $\delta=$ 128.00). Peak multiplicities are reported as follows: $s=$ singlet, $d=$ doublet, $t=$ triplet, $\mathrm{dd}=$ doublet of doublets, $\mathrm{td}=$ triplet of doublets, $\mathrm{m}=$ multiplet. High-resolution mass spectra (HRMS) were obtained by the mass spectrometry facility of the Technion. Reactions were monitored by gas chromatography spectrometry (GC) using an Agilent Technologies 7820A GC with an Agilent Technologies 19091J-413 (30 m $\times 0.3 \mathrm{~mm}$ ) column. $\mathrm{Ni}(\mathrm{acac})_{2}$ was recrystallized from toluene and dried under reduced pressure. THF was dried from Pure-Solv ${ }^{\oplus}$ Purification System (Innovative Technology ${ }^{\odot}$ ). Toluene was distilled over sodium/benzophenone prior to use. All other commercially obtained reagents were used as received.

\section{Preparation of neopentyl arylboronic esters}

2-(4-Methylphenyl)-5,5-dimethyl-1,3,2-dioxaborinane (2a, CAS 380481-66-3) ${ }^{1}$, 2phenyl-5,5-dimethyl-1,3,2-dioxaborinane (2b, CAS 5123-13-7) ${ }^{1}$, 2-(2-methylphenyl)5,5-dimethyl-1,3,2-dioxaborinane (2c, CAS 91994-11-5) ${ }^{1}$, 2-(4-trifluoromethylphenyl)5,5-dimethyl-1,3,2-dioxaborinane (2d, CAS 501374-30-7) ${ }^{1}$, 2-(4-biphenyl)-5,5dimethyl-1,3,2-dioxaborinane (2e, CAS 5123-05-7)2, 2-(2-naphthyl)-5,5-dimethyl1,3,2-dioxaborinane (2f, CAS 627906-96-1) ${ }^{1}$, 2-(1-naphthyl)-5,5-dimethyl-1,3,2dioxaborinane (2g, CAS 22871-77-8) ${ }^{1}$, 2-(4-methoxyphenyl)-5,5-dimethyl-1,3,2dioxaborinane (2h, CAS 213596-33-9)1, 2-(3-methoxyphenyl)-5,5-dimethyl-1,3,2dioxaborinane (2i, CAS 1003858-50-1)4, 2-(benzo[d][1,3]dioxol-5-yl)-5,5-dimethyl1,3,2-dioxaborinane (2j, CAS: 94838-83-2) 33 4-(5,5-dimethyl-1,3,2-dioxaborinan-2-yl)$\mathrm{N}, \mathrm{N}$-dimethylaniline $\quad\left(\mathbf{2 k}, \quad \mathrm{CAS} \quad\right.$ 95752-87-7) ${ }^{1}, \quad$ 1-methyl-5-(5,5-dimethyl-1,3,2dioxaborinan-2-yl)-1H-indole (2m, CAS 916518-57-5) ${ }^{5}, \quad$ 2-(4-fluorophenyl)-5,5dimethyl-1,3,2-dioxaborinane (2n, CAS: 225916-39-2)1, 2-(furan-2-yl)-5,5-dimethyl- 
1,3,2-dioxaborinane (2o, CAS: 941320-88-3) ${ }^{4}, \quad$ 2-mesityl-5,5-dimethyl-1,3,2dioxaborinane (2p, CAS 214360-78-8) , 2-(4-chlorophenyl)-5,5-dimethyl-1,3,2dioxaborinane (2q, CAS 827605-29-8) 2, 2-(3-nitrophenyl)-5,5-dimethyl-1,3,2dioxaborinane (2r, CAS 585524-79-4) ${ }^{6}$ and 2-(thiophen-3-yl)-5,5-dimethyl-1,3,2dioxaborinane (2t, CAS 905966-46-3) ${ }^{4}$ were prepared according to the literature procedure by the reaction of the corresponding boronic acids with 2,2dimethylpropane-1,3-diol. 5,5-Dimethyl-2-(3-pyridyl)-1,3,2-dioxaborinane (2s, CAS 845885-86-1) was purchased from Apollo Scientific and used as received.<smiles>CN(C)C(=O)c1cccc(B2OCC(C)(C)CO2)c1</smiles>

2I

\section{N,N-Dimethyl-3-(5,5-dimethyl-1,3,2-dioxaborinan-2-yl)benzamide (2I, CAS 2266574-} 36-9). 2,2-Dimethylpropane-1,3-diol (neopentyl glycol) (1.15 equiv., 0.35 g, 3.396 $\mathrm{mmol}$ ) was added to a stirred solution of 3-(dimethylcarbamoyl)phenylboronic acid (CAS 373384-14-6, $0.57 \mathrm{~g}, 2.953 \mathrm{mmol})$ in THF $(6 \mathrm{~mL})$, and the reaction mixture was stirred at room temperature for $16 \mathrm{~h}$. The reaction mixture was then concentrated under reduced pressure. The crude residue was purified by column chromatography on silica gel (petroleum ether / ethyl acetate $=3: 1$ to $1: 2$ ) to obtain the desired product 2l as a white soild (0.47 g, 61\%). 2l: ${ }^{1} \mathrm{H}$ NMR (400 MHz, $\left.\mathrm{CDCl}_{3}\right) \delta 7.80(\mathrm{~s}, 1 \mathrm{H} ; \mathrm{Ar}-\mathrm{H}), 7.79$ (d, J = 8.6 Hz, 1H; Ar-H), $7.44(\mathrm{~d}, J=7.6 \mathrm{~Hz}, 2 \mathrm{H} ; \mathrm{Ar}-\mathrm{H}), 7.35$ (t, J = 7.5 Hz, 1H; Ar-H), 3.73 (s, 4H), $3.07\left(\mathrm{~s}, 3 \mathrm{H} ; \mathrm{NCH}_{3}\right), 2.94\left(\mathrm{~s}, 3 \mathrm{H} ; \mathrm{NCH}_{3}\right), 0.98\left(\mathrm{~s}, 3 \mathrm{H} ; \mathrm{CH}_{3}\right) ;{ }^{13} \mathrm{C} \mathrm{NMR}(100 \mathrm{MHz}$, $\left.\mathrm{CDCl}_{3}\right) \delta 172.2(\mathrm{C}), 135.5(\mathrm{C}), 134.8(\mathrm{CH}), 132.2(\mathrm{CH}), 129.9(\mathrm{CH}), 127.5(\mathrm{CH}), 72.3\left(\mathrm{CH}_{2}\right.$ $\times 2)$, $39.6\left(\mathrm{NCH}_{3}\right), 31.8\left(\mathrm{NCH}_{3}\right), 31.8(\mathrm{C}), 21.8\left(\mathrm{CH}_{3} \times 2\right)$ (carbon atom directly attached to the boron atom could not be observed); $\mathrm{HRMS}(\mathrm{APCl}): \mathrm{m} / z$ calcd for $\mathrm{C}_{14} \mathrm{H}_{21} \mathrm{BNO}_{3}$ ([M $\left.+\mathrm{H}]^{+}\right): 262.1609$, found 262.1601. 


\section{Preparation of alkenyl methyl ethers}

1-Methoxyundec-1-ene $\quad(1 \mathrm{a})^{7}, \quad$ 2-cyclohexyl-1-methoxyethylene $\quad(\mathbf{1 c})^{8}, \quad$ 1methoxydodeca-1,11-diene (1d) ${ }^{9}$, 1-methoxy-4,8-dimethylnona-1,7-diene $(\mathbf{1 e})^{10},(4-$ methoxybut-3-en-1-yl)benzene $\quad(\mathbf{1 f})^{10}, \quad$ (2-methoxyethenyl)benzene $\quad(\mathbf{1 i})^{7}, \quad 4-(2-$ methoxyethenyl)- $N, N$-dimethylaniline $(\mathbf{1} \mathbf{j})^{7}$, methyl 4-(2-methoxyvinyl)benzoate $(\mathbf{1 k})^{11}$, 2-methoxy-6-(2-methoxyvinyl)naphthalene $\quad(\mathbf{1 1})^{12}, \quad$ 1-(1-methoxyprop-1-en-2yl)benzene $(\mathbf{1} \mathbf{m})^{13}$ and 1,2,3-trimethoxy-5-(2-methoxyvinyl)benzene $(\mathbf{1 n})^{14}$ were prepared according to the literature procedure by Wittig olefination. 2,3Dihydrofuran (1h, CAS 1191-99-7) was purchased from Acros Organics and used as received.<smiles>CCCCC=COC</smiles>

$1 b$

1-Methoxyhex-1-ene (1b). ${ }^{15}$ To a suspension of potassium tert-butoxide (1.35 g, 12.0 $\mathrm{mmol}$ ) in $\mathrm{Et}_{2} \mathrm{O}(20 \mathrm{~mL})$, (methoxymethyl)triphenylphosphonium chloride (3.94 g, 11.5 mmol) was added at room temperature, and the mixture was stirred for $45 \mathrm{~min}$. Valeraldehyde $(1.06 \mathrm{~mL}, 10.0 \mathrm{mmol})$ was then added slowly and the mixture was stirred for $12 \mathrm{~h}$ at room temperature. The precipitate was removed by filtration and the filtrate was concentrated under reduced pressure (Note: 1-methoxyhex-1-ene is volatile, bp $110-118{ }^{\circ} \mathrm{C}$ (760 torr $)^{15}$. The crude product was purified by column chromatography on silica gel (pentane/ether $=100: 0$ to $95: 5$ ) to give product $\mathbf{1 b}$ as a 51:49 (E:Z) mixture isomers as a clear oil $(0.88 \mathrm{~g}, 7.7 \mathrm{mmol} 77 \%) .(E)-\mathbf{1 b}:{ }^{1} \mathrm{H}$ NMR (400 $\left.\mathrm{MHz}, \mathrm{C}_{6} \mathrm{D}_{6}\right) \delta 6.31(\mathrm{~d}, J=12.6 \mathrm{~Hz}, 1 \mathrm{H}$; olefin-H), $4.64(\mathrm{dt}, J=12.6,7.3 \mathrm{~Hz}, 1 \mathrm{H}$; olefin-H), $3.18(\mathrm{~s}, 3 \mathrm{H} ; \mathrm{OMe}), 1.86-1.84(\mathrm{~m}, 2 \mathrm{H}), 1.38-1.34(\mathrm{~m}, 2 \mathrm{H}), 1.28-1.23(\mathrm{~m}, 2 \mathrm{H}), 0.88(\mathrm{t}, \mathrm{J}$ $=7.1 \mathrm{~Hz}, 3 \mathrm{H}) ;{ }^{13} \mathrm{CNMR}\left(100 \mathrm{MHz}, \mathrm{C}_{6} \mathrm{D}_{6}\right) \delta 147.7(\mathrm{CH}), 102.6(\mathrm{CH}), 55.3\left(\mathrm{CH}_{3}\right), 33.4\left(\mathrm{CH}_{2}\right)$, 
$27.8\left(\mathrm{CH}_{2}\right), 22.4\left(\mathrm{CH}_{2}\right), 14.1\left(\mathrm{CH}_{3}\right) .(\mathrm{Z})-1 \mathrm{~b}:{ }^{1} \mathrm{H} \mathrm{NMR}\left(400 \mathrm{MHz}, \mathrm{C}_{6} \mathrm{D}_{6}\right) \delta 5.70(\mathrm{dt}, J=6.2$, $1.4 \mathrm{~Hz}, 1 \mathrm{H}$; olefin-H), 4.37 (td, J = 7.3, $6.2 \mathrm{~Hz}, 1 \mathrm{H}$; olefin-H), 3.13 (s, 3H; OMe), 2.27 (qd, $J=7.3,1.4 \mathrm{~Hz}, 2 \mathrm{H}), 1.37-1.32(\mathrm{~m}, 2 \mathrm{H}), 1.27-1.23(\mathrm{~m}, 2 \mathrm{H}), 0.86(\mathrm{t}, J=7.0 \mathrm{~Hz}, 3 \mathrm{H}) ;{ }^{13} \mathrm{C}$ NMR (100 MHz, $\left.\mathrm{C}_{6} \mathrm{D}_{6}\right) \delta 146.5(\mathrm{CH}), 106.9(\mathrm{CH}), 58.9\left(\mathrm{CH}_{3}\right), 32.4\left(\mathrm{CH}_{2}\right), 24.1\left(\mathrm{CH}_{2}\right), 22.7$ $\left(\mathrm{CH}_{2}\right), 14.2\left(\mathrm{CH}_{3}\right)$.<smiles>COC=CC(C)c1ccccc1</smiles>

$1 \mathrm{~g}$

1-(4-Methoxybut-3-enyl)benzene (1g). The above general procedure was followed using (methoxymethyl)triphenylphosphonium chloride (3.94 g, $11.5 \mathrm{mmol})$, potassium tert-butoxide $(1.35 \mathrm{~g}, 12.0 \mathrm{mmol})$ and 2-phenylpropionaldehyde $(1.34 \mathrm{~mL}$, $10.0 \mathrm{mmol}$ ). The crude product was purified by column chromatography on silica gel (petroleum ether/ether $=10: 1)$ to give product $1 \mathrm{~g}$ as a $60: 40(E: Z)$ mixture isomers as a clear oil (1.17 g, $7.2 \mathrm{mmol} 72 \%)$. As (E)- and (Z)-1g are inseparable, NMR and MS data are given for the mixture of the two isomers. $(E)-1 \mathrm{~g}:{ }^{1} \mathrm{H}$ NMR $\left(400 \mathrm{MHz}, \mathrm{C}_{6} \mathrm{D}_{6}\right) \delta 7.16-$ $7.13(\mathrm{~m}, 4 \mathrm{H} ; \mathrm{Ar}-\mathrm{H}), 7.08-7.04(\mathrm{~m}, 1 \mathrm{H} ; \mathrm{Ar}-\mathrm{H}), 6.33(\mathrm{~d}, \mathrm{~J}=12.7 \mathrm{~Hz}, 1 \mathrm{H}$; olefin-H), 4.82 (dd, $J=12.7,7.8 \mathrm{~Hz}, 1 \mathrm{H}$; olefin-H), 3.26-3.19 (m, 2H), $3.10(\mathrm{~s}, 3 \mathrm{H} ; \mathrm{OMe}), 1.24(\mathrm{~d}, J=$ $7.0 \mathrm{~Hz}, 3 \mathrm{H}) ;{ }^{13} \mathrm{C} \mathrm{NMR}\left(100 \mathrm{MHz}, \mathrm{C}_{6} \mathrm{D}_{6}\right) \delta 147.3(\mathrm{C}$ and $\mathrm{CH}), 128.6(\mathrm{CH} \times 2), 127.2(\mathrm{CH} \times$ 2), $126.2(\mathrm{CH}), 108.4(\mathrm{CH}), 55.3\left(\mathrm{CH}_{3}\right), 38.8(\mathrm{CH}), 22.8\left(\mathrm{CH}_{3}\right)$; HRMS (APCI): $\mathrm{m} / z$ calcd for $\mathrm{C}_{11} \mathrm{H}_{15} \mathrm{O}\left([\mathrm{M}+\mathrm{H}]^{+}\right)$: 163.1117 , found 163.1112. (Z)-1b: ${ }^{1} \mathrm{H} N M R\left(400 \mathrm{MHz}, \mathrm{C}_{6} \mathrm{D}_{6}\right) \delta$ 7.29 (d, J = 7.5 Hz, 2H; Ar-H), 7.20-7.14 (m, 2H; Ar-H), 7.07-7.04 (m, 1H; Ar-H), 5.59 (d, $J=6.2 \mathrm{~Hz}, 1 \mathrm{H}$; olefin-H), $4.53(\mathrm{dd}, J=9.3,6.2 \mathrm{~Hz}, 1 \mathrm{H}$; olefin- $\mathrm{H}), 4.22-4.14(\mathrm{~m}, 2 \mathrm{H})$, 3.06 (s, 3H; OMe), 1.36 (d, J = 7.1 Hz, 3H); ${ }^{13} \mathrm{C}$ NMR (100 MHz, $\left.\mathrm{C}_{6} \mathrm{D}_{6}\right) \delta 147.5(\mathrm{C}), 145.4$ (CH), $128.6(\mathrm{CH} \times 2), 127.3(\mathrm{CH} \times 2), 126.0(\mathrm{CH}), 112.4(\mathrm{CH}), 59.1\left(\mathrm{CH}_{3}\right), 34.9(\mathrm{CH}), 22.6$ $\left(\mathrm{CH}_{3}\right) ; \mathrm{HRMS}(\mathrm{APCl}): \mathrm{m} / z$ calcd for $\mathrm{C}_{11} \mathrm{H}_{15} \mathrm{O}\left([\mathrm{M}+\mathrm{H}]^{+}\right): 163.1117$, found 163.1112 . 


\section{Optimization of nickel-catalyzed Suzuki-Miyaura cross-}

\section{coupling of alkenyl ether $1 \mathrm{a}$}

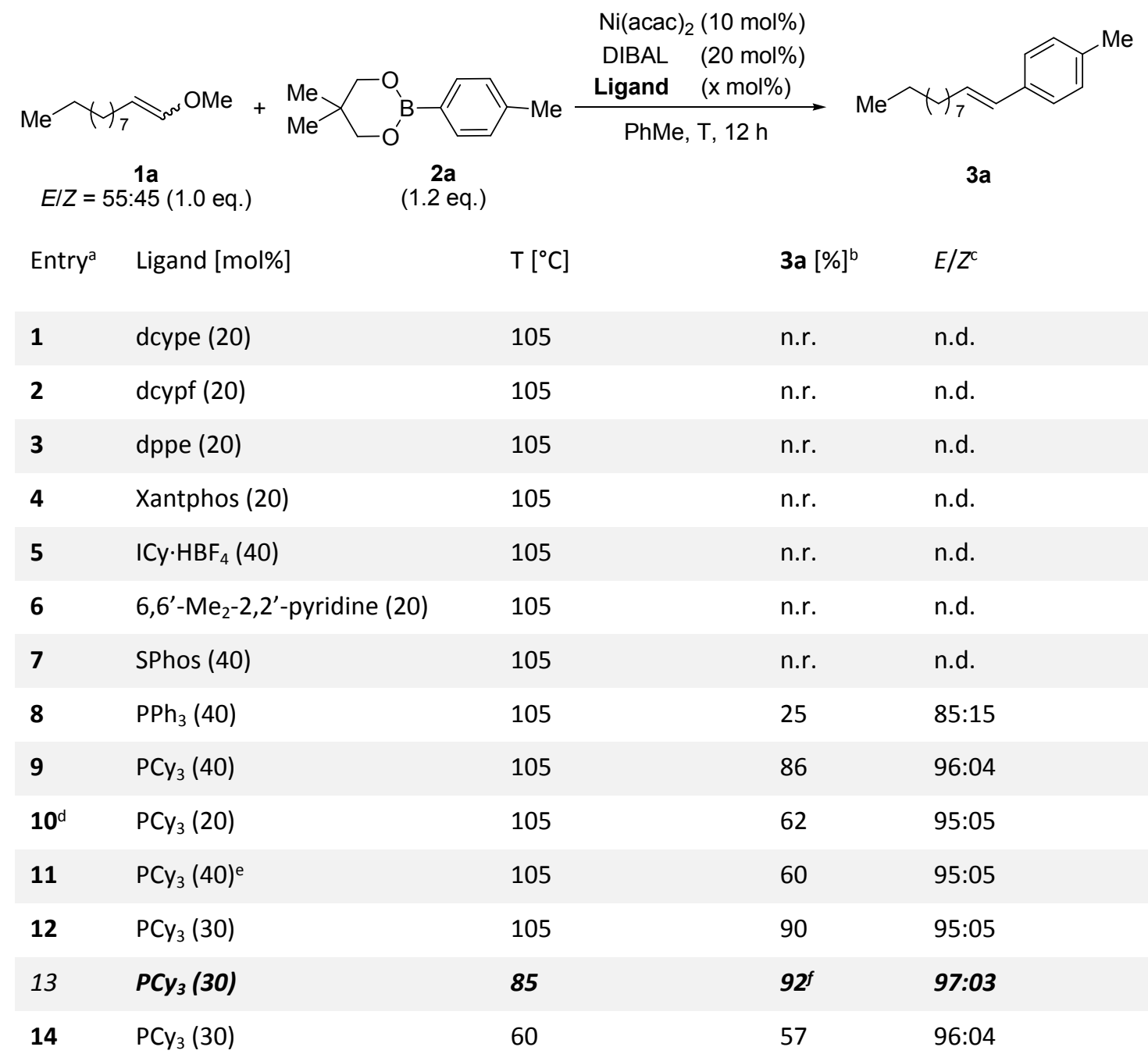

${ }^{a}$ All reactions were carried out using $1 \mathrm{a}(0.27 \mathrm{mmol})$ and $2 \mathrm{a}(0.32 \mathrm{mmol}, 120 \mathrm{~mol} \%)$ in $1.4 \mathrm{~mL}$ of toluene.

${ }^{b}$ Yields determined by analysis of the unpurified mixture of products by ${ }^{1} \mathrm{H}$ NMR with an internal standard in chloroform-D. ' Ratio determined by analysis of the unpurified mixture of products by ${ }^{1} \mathrm{H}$ NMR. ${ }^{d} \mathrm{Ni}(\mathrm{acac})_{2}$ (5.0 mol\%), DIBAL (10 mol\%). e CsF (120 mol\%). ${ }^{f}$ Yields of isolated products after purification by column chromatography. 


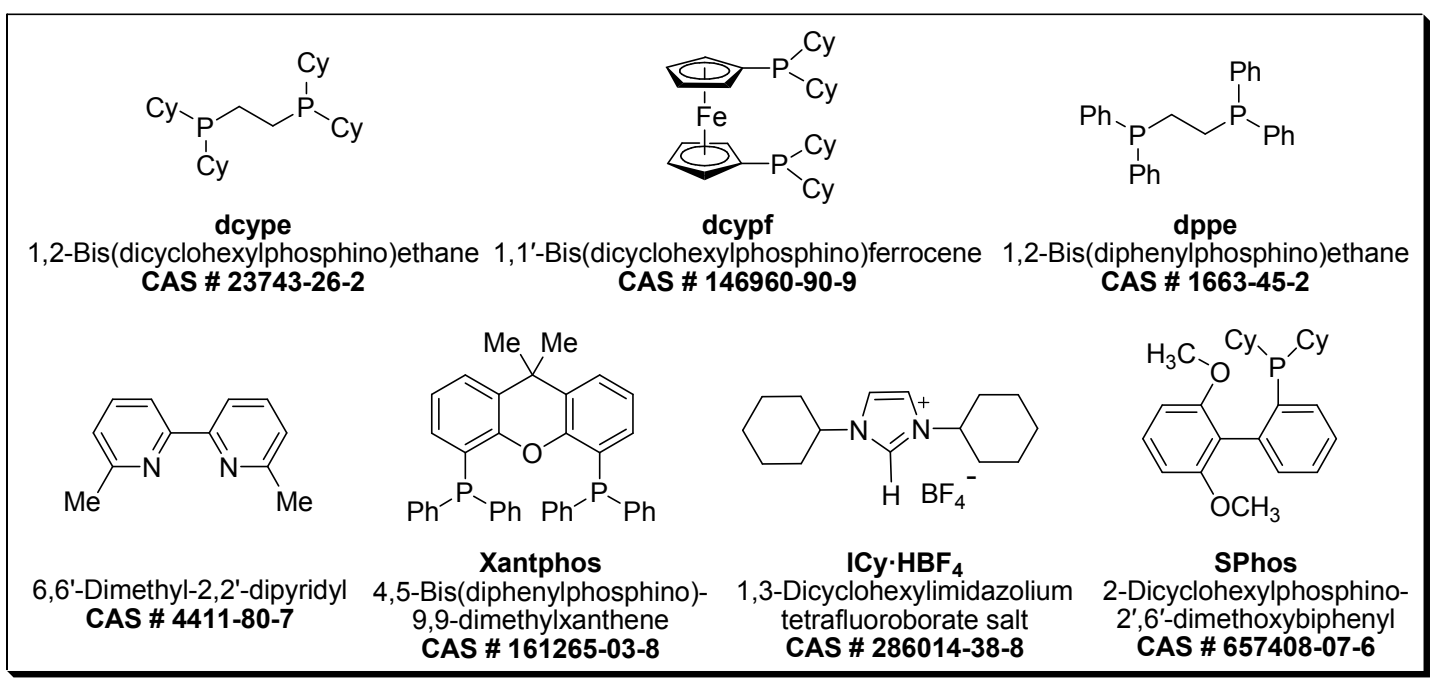

General procedure for the optimization of nickel-catalyzed Suzuki-Miyaura crosscoupling of alkenyl ethers. To a flame-dried Schlenk flask equipped with a rubber septum and magnetic stir bar was purged with argon, then $\mathrm{Ni}(\mathrm{acac})_{2}(10 \mathrm{~mol} \%, 0.027$ mmol, $7 \mathrm{mg}$ ) and the specified ligand (dcype, dcypf, dppe, Xantphos, ICy.HBF 4 , 6,6'dimethyl-2,2'-dipyridyl, SPhos, $\mathrm{PPh}_{3}$ or $\mathrm{PCy}_{3}, 20-40$ mol\%) were added. The flask was evacuated and backfilled with argon (3 times), and toluene $(1.0 \mathrm{~mL})$ was added by a syringe. Diisobutylaluminum hydride (DIBAL, $20 \mathrm{~mol} \%, 1.0 \mathrm{M}$ in hexane, $0.054 \mathrm{mmol}$ ) was then added dropwise with a syringe at ambient temperature over 2 min. After stirring at ambient temperature for $15 \mathrm{~min}$, a solution of 5,5-dimethyl-2-p-tolyl-1,3,2dioxaborinane 2a (1.2 equiv., $0.326 \mathrm{mmol}, 67 \mathrm{mg}$ ) in toluene $(0.4 \mathrm{~mL}$ ) and alkenyl methyl ether 1a (1.0 equiv., $0.272 \mathrm{mmol}, 50 \mathrm{mg}$ ) were added dropwise to the reaction mixture via syringe under argon. The resulting mixture was heated at the indicated temperature for $12 \mathrm{~h}$. After being cooled to ambient temperature, the reaction mixture was quenched with an aqueous saturated solution of $\mathrm{NH}_{4} \mathrm{Cl}$ and diluted with $\mathrm{Et}_{2} \mathrm{O}$. The combined organic phase was filtered through a pad of silica gel with copious washings by $\mathrm{Et}_{2} \mathrm{O}$ and concentrated. 2-Naphthaldehyde was then added as an internal standard and a small aliquot chloroform- $d_{1}$ was used for ${ }^{1} \mathrm{H}$ NMR analysis to evaluate the yield and $E / Z$ ration of styrene $3 a$. The crude mixture with internal standard was 
allowed to be further purified by column chromatography on silica gel (eluent, petroleum ether/toluene $=20: 1)$ to afford isolated yield of $\mathbf{3 a}$.

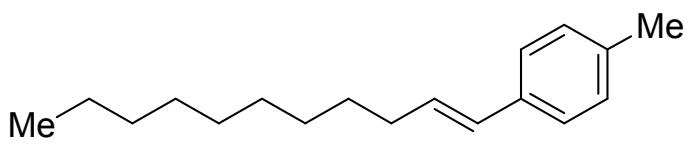

$(E)-3 a$

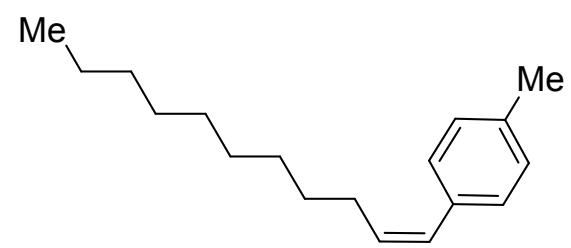

(Z)-3a

(E)- and (Z)-1-Methyl-4-(undec-1-enyl)benzene (3a). ${ }^{7}$ The representative example for the synthesis of styrene 3a was prepared according to the general procedure described in entry 13 of table 1 . Standard work-up afforded crude $3 a$ as a mixture of $(E)$ - and $(Z)-3 a$ in a ratio $E / Z=97: 3$, and then purification by column chromatography on silica gel (eluent, petroleum ether/toluene $=20: 1$ ) yielded the title compound $\mathbf{3 a}$ as a clear oil (61 mg, $0.250 \mathrm{mmol}, 92 \%$ yield). The $(E)$ - and (Z)-isomers were partially separated by column chromatography. (E)-3a: ${ }^{1} \mathrm{H} N M R\left(400 \mathrm{MHz}, \mathrm{CDCl}_{3}\right) \delta 7.23(\mathrm{~d}, J=$ $8.1 \mathrm{~Hz}, 2 \mathrm{H} ; \mathrm{Ar}-\mathrm{H}), 7.09$ (d, J = 8.0 Hz, 2H; Ar-H), 6.34 (d, J = $15.8 \mathrm{~Hz}, 1 \mathrm{H}$; olefin-H), 6.18 $(\mathrm{dt}, J=15.8,6.9 \mathrm{~Hz}, 1 \mathrm{H}$; olefin-H), $2.32(\mathrm{~s}, 3 \mathrm{H}), 2.17(\mathrm{qd}, J=7.2,1.0 \mathrm{~Hz}, 2 \mathrm{H}), 1.46-1.44$ (m, 2H), 1.31-1.28 (m, 12H), $0.89(\mathrm{t}, J=7.2 \mathrm{~Hz}, 3 \mathrm{H}) ;{ }^{13} \mathrm{C} \mathrm{NMR}\left(100 \mathrm{MHz}, \mathrm{CDCl}_{3}\right) \delta 136.4$ (C), $135.2(\mathrm{C}), 130.2(\mathrm{CH}), 129.5(\mathrm{CH}), 129.1(\mathrm{CH} \times 2), 125.8(\mathrm{CH} \times 2), 33.0\left(\mathrm{CH}_{2}\right), 31.9$ $\left(\mathrm{CH}_{2}\right), 29.6\left(\mathrm{CH}_{2} \times 2\right), 29.5\left(\mathrm{CH}_{2}\right), 29.3\left(\mathrm{CH}_{2}\right), 29.2\left(\mathrm{CH}_{2}\right), 22.7\left(\mathrm{CH}_{2}\right), 22.1\left(\mathrm{CH}_{3}\right), 14.0$ $\left(\mathrm{CH}_{3}\right) \cdot(\mathrm{Z})-3 \mathrm{a}:{ }^{1} \mathrm{H}$ NMR $\left(400 \mathrm{MHz}, \mathrm{CDCl}_{3}\right) \delta 7.16(\mathrm{~d}, J=8.1 \mathrm{~Hz}, 2 \mathrm{H} ; \mathrm{Ar}-\mathrm{H}), 7.11(\mathrm{~d}, J=8.0$ $\mathrm{Hz}, 2 \mathrm{H} ; \mathrm{Ar}-\mathrm{H}), 6.35(\mathrm{~d}, J=11.6 \mathrm{~Hz}, 1 \mathrm{H}$; olefin-H), $5.60(\mathrm{dt}, J=11.6,7.2 \mathrm{~Hz}, 1 \mathrm{H}$; olefinH), $2.33(\mathrm{~s}, 3 \mathrm{H}), 2.32(\mathrm{q}, J=7.2,1.0 \mathrm{~Hz}, 2 \mathrm{H}), 1.44-1.38(\mathrm{~m}, 2 \mathrm{H}), 1.31-1.24(\mathrm{~m}, 12 \mathrm{H})$, $0.86(t, J=6.8 \mathrm{~Hz}, 3 \mathrm{H}) ;{ }^{13} \mathrm{C} \mathrm{NMR}\left(100 \mathrm{MHz}, \mathrm{CDCl}_{3}\right) \delta 136.0(\mathrm{C}), 135.0(\mathrm{C}), 132.6(\mathrm{CH})$, $128.8(\mathrm{CH} \times 2), 128.7(\mathrm{CH} \times 2), 128.5(\mathrm{CH}), 31.9\left(\mathrm{CH}_{2}\right), 30.0\left(\mathrm{CH}_{2}\right), 29.6\left(\mathrm{CH}_{2}\right), 29.5\left(\mathrm{CH}_{2}\right)$, $29.4\left(\mathrm{CH}_{2}\right), 29.3\left(\mathrm{CH}_{2}\right), 28.7\left(\mathrm{CH}_{2}\right), 22.7\left(\mathrm{CH}_{2}\right), 22.1\left(\mathrm{CH}_{3}\right), 14.1\left(\mathrm{CH}_{3}\right)$. Spectral data of $(E)$ - and (Z)-3a matched reported literature values. ${ }^{7}$ 


\section{Nickel-catalyzed Suzuki-Miyaura cross-coupling of alkenyl ethers with different neopentyl boronic esters}

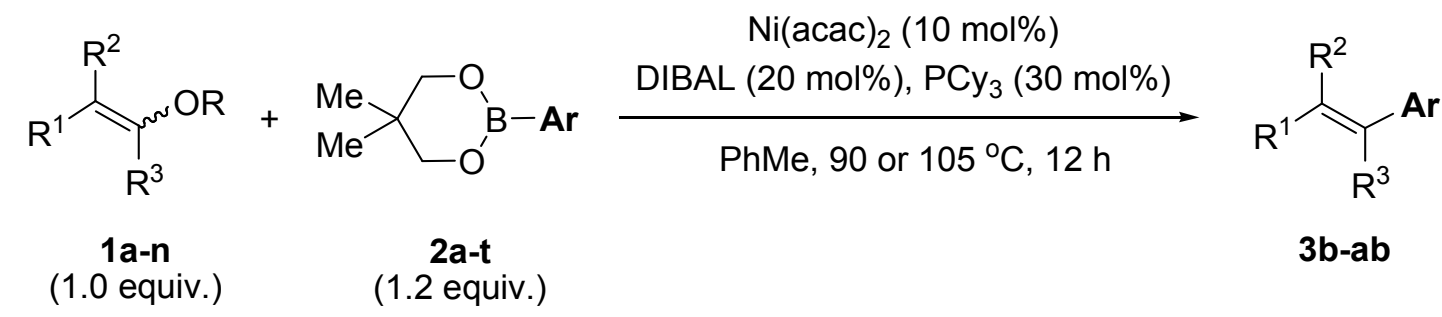

General procedure for nickel-catalyzed Suzuki-Miyaura cross-coupling of alkenyl ethers with different neopentyl boronic esters. To a flame-dried Schlenk flask equipped with a rubber septum and stirrer bar was purged with argon, then $\mathrm{Ni}(\mathrm{acac})_{2}$ (10 mol\%, $0.027 \mathrm{mmol}, 7 \mathrm{mg}$ ) and $\mathrm{PC}_{3}(30 \mathrm{~mol} \%, 0.082 \mathrm{mmol}, 23 \mathrm{mg}$ ) were added. The flask evacuated and backfilled with argon ( 3 times), and then toluene $(1.0 \mathrm{~mL})$ was added by a syringe. Diisobutylaluminum hydride (DIBAL, $20 \mathrm{~mol} \%, 1.0 \mathrm{M}$ in hexane, $0.054 \mathrm{mmol}$ ) was then added dropwise with a syringe at ambient temperature over 2 min. After stirring at ambient temperature for $15 \mathrm{~min}$, a solution of the corresponding neopentyl boronic ester (1.2 equiv., $0.326 \mathrm{mmol}$ ) in toluene $(0.4 \mathrm{~mL})$ and alkenyl methyl ether (1.0 equiv., $0.272 \mathrm{mmol}$ ) were added to the reaction mixture via syringe under argon. The resulting mixture was heated at the indicated temperature for $12 \mathrm{~h}$. After being cooled to ambient temperature, the reaction mixture was quenched with an aqueous saturated solution of $\mathrm{NH}_{4} \mathrm{Cl}$ and diluted with $\mathrm{Et}_{2} \mathrm{O}$. The combined organic phase was filtered through a pad of silica gel with copious washings by $\mathrm{Et}_{2} \mathrm{O}$ and concentrated. The crude product was purified by column chromatography on silica gel to afford isolated yield of the desired styrene derivative. 


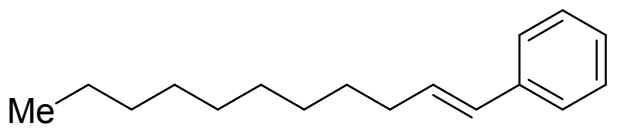

$(E)-3 \mathbf{b}$

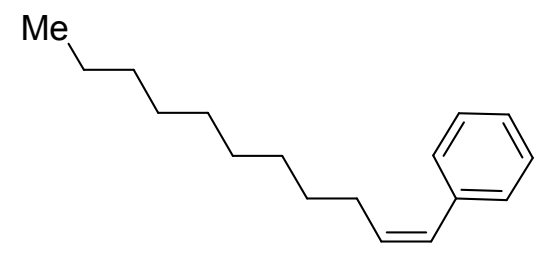

(Z)-3b

$(E)$ - and (Z)-1-(Dec-1-enyl)benzene (3b). ${ }^{16}$ Compounds $(E)$ - and (Z)-3b were synthesized from 1-methoxyundec-1-ene $1 \mathrm{a}(E / Z=55: 45,0.272 \mathrm{mmol}, 50 \mathrm{mg})$ and 5,5dimethyl-2-phenyl-1,3,2-dioxaborinane $\mathbf{2 b}(0.326 \mathrm{mmol}, 62 \mathrm{mg})$ according to general procedure. Standard work-up afforded crude $\mathbf{3 b}$ as a mixture of $(E)$ - and (Z)-3b in a ratio $E / Z=96: 4$, and then purification by column chromatography on silica gel (eluent, petroleum ether/toluene $=20: 1$ ) yielded the title compound $\mathbf{3 b}$ as a clear oil $(51 \mathrm{mg}$, $0.221 \mathrm{mmol}, 82 \%$ yield). The (E)- and (Z)-isomers were partially separated by column chromatography. (E)-3b: ${ }^{1} \mathrm{H}$ NMR $\left(400 \mathrm{MHz}, \mathrm{CDCl}_{3}\right) \delta 7.34-7.25(\mathrm{~m}, 4 \mathrm{H}, \mathrm{Ph}-\mathrm{H}), 7.19-$ $7.15(\mathrm{~m}, 1 \mathrm{H}, \mathrm{Ph}-\mathrm{H}), 6.36(\mathrm{~d}, J=15.8 \mathrm{~Hz}, 1 \mathrm{H}$; olefin-H), $6.24(\mathrm{dt}, J=15.8,6.8 \mathrm{~Hz}, 1 \mathrm{H})$, $2.19(\mathrm{qd}, J=7.9,1.2 \mathrm{~Hz}, 2 \mathrm{H}), 1.47-1.42(\mathrm{~m}, 2 \mathrm{H}), 1.30-1.27(\mathrm{~m}, 12 \mathrm{H}), 0.87(\mathrm{t}, J=6.9 \mathrm{~Hz}$, $3 \mathrm{H}) ;{ }^{13} \mathrm{C}$ NMR $\left(100 \mathrm{MHz}, \mathrm{CDCl}_{3}\right) \delta 138.0(\mathrm{C}), 131.3(\mathrm{CH}), 129.6(\mathrm{CH}), 128.4(\mathrm{CH} \times 2)$, 126.7 (CH), $125.9(\mathrm{CH} \times 2), 33.1\left(\mathrm{CH}_{2}\right), 31.9\left(\mathrm{CH}_{2}\right), 29.5\left(\mathrm{CH}_{2}\right), 29.4\left(\mathrm{CH}_{2}\right), 29.3\left(\mathrm{CH}_{2} \times 2\right)$, $29.2\left(\mathrm{CH}_{2}\right), 22.7\left(\mathrm{CH}_{2}\right), 14.2\left(\mathrm{CH}_{3}\right)$; HRMS (APCl): $\mathrm{m} / \mathrm{z}$ calcd for $\mathrm{C}_{17} \mathrm{H}_{27}\left([\mathrm{M}+\mathrm{H}]^{+}\right)$: 231.2107, found 231.2093. (Z)-3b: ${ }^{1} \mathrm{H}$ NMR (400 MHz, $\left.\mathrm{CDCl}_{3}\right) \delta 7.33-7.24(\mathrm{~m}, 4 \mathrm{H}, \mathrm{Ph}-$ H), 7.21-7.17 (m, 1H, Ph-H), $6.38(\mathrm{~d}, J=11.5 \mathrm{~Hz}, 1 \mathrm{H}$; olefin-H), $5.65(\mathrm{dt}, J=11.6,7.2$ $\mathrm{Hz}, 1 \mathrm{H}), 2.31(\mathrm{qd}, J=7.4,1.6 \mathrm{~Hz}, 2 \mathrm{H}), 1.46-1.39(\mathrm{~m}, 2 \mathrm{H}), 1.29-1.23(\mathrm{~m}, 12 \mathrm{H}), 0.86(\mathrm{t}, J$ $=7.0 \mathrm{~Hz}, 3 \mathrm{H}) ;{ }^{13} \mathrm{C} \mathrm{NMR}\left(100 \mathrm{MHz}, \mathrm{CDCl}_{3}\right) \delta 137.8(\mathrm{C}), 133.3(\mathrm{CH}), 128.7(\mathrm{CH} \times 2), 128.6$ (CH), $128.1(\mathrm{CH} \times 2), 126.4(\mathrm{CH}), 31.9\left(\mathrm{CH}_{2}\right), 30.0\left(\mathrm{CH}_{2}\right), 29.7\left(\mathrm{CH}_{2}\right), 29.6\left(\mathrm{CH}_{2}\right), 29.5$ $\left(\mathrm{CH}_{2}\right), 29.3\left(\mathrm{CH}_{2}\right), 28.6\left(\mathrm{CH}_{2}\right), 22.7\left(\mathrm{CH}_{2}\right), 14.1\left(\mathrm{CH}_{3}\right)$; HRMS (APCl): $\mathrm{m} / z$ calcd for $\mathrm{C}_{17} \mathrm{H}_{27}$ $\left([\mathrm{M}+\mathrm{H}]^{+}\right): 231.2107$, found 231.2093. Spectral data of $(E)-$ and $(Z)-3 \mathbf{b}$ matched reported literature values. ${ }^{16}$ 


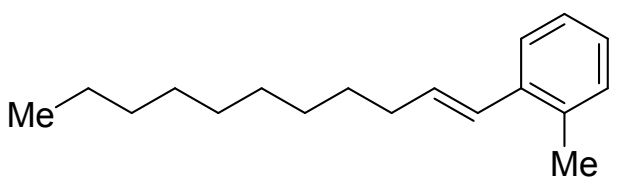

$(E)-3 c$

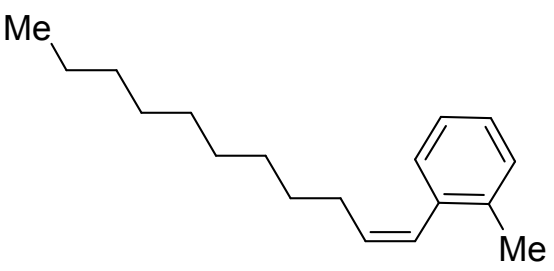

$(Z)-3 c$

$(E)$ - and (Z)-1-Methyl-2-(undec-1-enyl)benzene (3c). Compounds (E)- and (Z)-3c were synthesized from 1-methoxyundec-1-ene $1 \mathrm{a}(E / Z=55: 45,0.272 \mathrm{mmol}, 50 \mathrm{mg})$ and 5,5dimethyl-2-o-tolyl-1,3,2-dioxaborinane $2 \mathrm{c}(0.326 \mathrm{mmol}, 67 \mathrm{mg})$ according to general procedure at $105^{\circ} \mathrm{C}$. Standard work-up afforded crude $3 \mathrm{c}$ as a mixture of $(E)$ - and $(Z)$ $3 c$ in a ratio $E / Z=92: 8$, and then purification by column chromatography on silica gel (eluent, petroleum ether/toluene $=20: 1$ ) yielded the title compound $3 \mathrm{c}$ as a clear oil (59 $\mathrm{mg}, 0.242 \mathrm{mmol}, 89 \%$ yield). The (E)- and (Z)-isomers were partially separated by column chromatography. (E)-3c: ${ }^{1} \mathrm{H} N M R\left(400 \mathrm{MHz}, \mathrm{CDCl}_{3}\right) \delta 7.39(\mathrm{~d}, \mathrm{~J}=6.9 \mathrm{~Hz}, 1 \mathrm{H}$; $\mathrm{Ar}-$ H), 7.14-7.11 (m, 3H; Ar-H), $6.55(\mathrm{~d}, J=15.7 \mathrm{~Hz}, 1 \mathrm{H}$; olefin-H), 6.09 (dt, $J=15.6,7.0$ $\mathrm{Hz}, 1 \mathrm{H}$; olefin-H), $2.32(\mathrm{~s}, 3 \mathrm{H}), 2.21(\mathrm{qd}, J=7.2,1.4 \mathrm{~Hz}, 2 \mathrm{H}), 1.48-1.43(\mathrm{~m}, 2 \mathrm{H}), 1.33-$ $1.27(\mathrm{~m}, 12 \mathrm{H}), 0.88(\mathrm{t}, J=7.2 \mathrm{~Hz}, 3 \mathrm{H}) ;{ }^{13} \mathrm{C} \mathrm{NMR}\left(100 \mathrm{MHz}, \mathrm{CDCl}_{3}\right) \delta 137.1(\mathrm{C}), 134.8(\mathrm{C})$, $132.6(\mathrm{CH}), 130.1(\mathrm{CH}), 127.5(\mathrm{CH}), 126.7(\mathrm{CH}), 126.0(\mathrm{CH}), 125.4(\mathrm{CH}), 33.3\left(\mathrm{CH}_{2}\right), 31.9$ $\left(\mathrm{CH}_{2}\right), 29.6\left(\mathrm{CH}_{2}\right), 29.5\left(\mathrm{CH}_{2} \times 2\right), 29.3\left(\mathrm{CH}_{2}\right), 29.2\left(\mathrm{CH}_{2}\right), 22.7\left(\mathrm{CH}_{2}\right), 19.8\left(\mathrm{CH}_{3}\right), 14.1$ $\left(\mathrm{CH}_{3}\right) ; \operatorname{HRMS}(\mathrm{APCl}): \operatorname{HRMS}(\mathrm{APCl}): \mathrm{m} / z$ calcd for $\mathrm{C}_{18} \mathrm{H}_{27}\left(\left[\left[\mathrm{M}-\mathrm{H}_{2}+\mathrm{H}\right]^{+}\right): 243.2067\right.$, found 243.2091. (Z)-3c: ${ }^{1} \mathrm{H} N M R\left(400 \mathrm{MHz}, \mathrm{CDCl}_{3}\right) \delta 7.16-7.11(\mathrm{~m}, 4 \mathrm{H} ; \mathrm{Ar}-\mathrm{H}), 6.40(\mathrm{~d}, \mathrm{~J}=11.5$ $\mathrm{Hz}, 1 \mathrm{H}$; olefin-H), 5.69 (dt, J=11.5, 7.4 Hz, 1H; olefin-H), $2.23(\mathrm{~s}, 3 \mathrm{H}), 2.13$ (qd, J = 7.4, 1.6 Hz, 2H), 1.47-1.43 (m, 2H), $1.32-1.23(\mathrm{~m}, 12 \mathrm{H}), 0.88(\mathrm{t}, J=7.2 \mathrm{~Hz}, 3 \mathrm{H}) ;{ }^{13} \mathrm{C} N M R$ $\left(100 \mathrm{MHz}, \mathrm{CDCl}_{3}\right) \delta 136.9(\mathrm{C}), 136.2(\mathrm{C}), 133.0(\mathrm{CH}), 129.7(\mathrm{CH}), 129.0(\mathrm{CH}), 127.7(\mathrm{CH})$, 126.6 (CH), $125.2(\mathrm{CH}), 31.9\left(\mathrm{CH}_{2}\right), 29.8\left(\mathrm{CH}_{2}\right), 29.6\left(\mathrm{CH}_{2}\right), 29.5\left(\mathrm{CH}_{2}\right), 29.3\left(\mathrm{CH}_{2}\right), 29.2$ $\left(\mathrm{CH}_{2}\right), 28.3\left(\mathrm{CH}_{2}\right), 22.7\left(\mathrm{CH}_{2}\right), 19.9\left(\mathrm{CH}_{3}\right), 14.1\left(\mathrm{CH}_{3}\right)$; HRMS (APCI): $\mathrm{m} / z$ calcd for $\mathrm{C}_{18} \mathrm{H}_{27}$ $\left(\left[\mathrm{M}-\mathrm{H}_{2}+\mathrm{H}\right]^{+}\right):$243.2067, found 243.2091. 


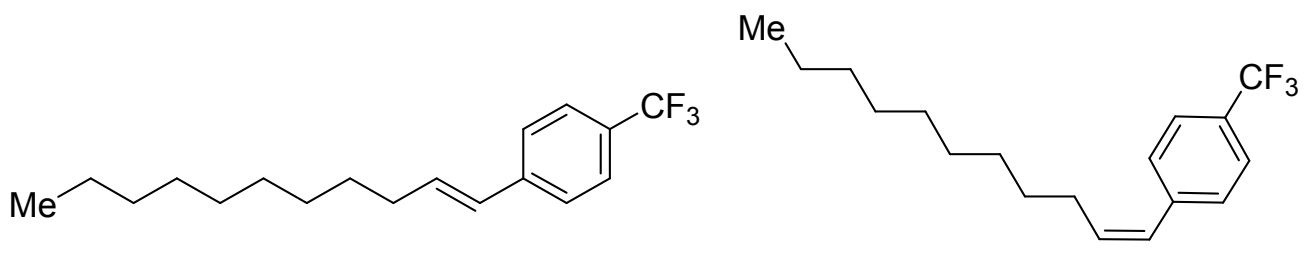

$(E)-3 \mathbf{d}$

(Z)-3d

(E)- and (Z)-1-(Trifluoromethyl)-4-(undec-1-enyl)benzene (3d). Compounds (E)- and (Z)-3d were synthesized from 1-methoxyundec-1-ene $1 \mathrm{a}(E / Z=55: 45,0.272 \mathrm{mmol}, 50$ mg) and 2-(4-(trifluoromethyl)phenyl)-5,5-dimethyl-1,3,2-dioxaborinane 2d (0.326 mmol, $84 \mathrm{mg}$ ) according to general procedure. Standard work-up afforded crude $\mathbf{3 d}$ as a mixture of $(E)$ - and $(Z)-3 \mathbf{d}$ in a ratio $E / Z=97: 3$, and then purification by column chromatography on silica gel (eluent, petroleum ether/toluene $=20: 1$ ) yielded the title compound $\mathbf{3 d}$ as a clear oil (69 $\mathrm{mg}, 0.231 \mathrm{mmol}, 85 \%$ yield). The (E)-isomer was partially separated by column chromatography. $(E)-3 \mathrm{~d}:{ }^{1} \mathrm{H}$ NMR $\left(400 \mathrm{MHz}, \mathrm{CDCl}_{3}\right) \delta$ $7.52(\mathrm{~d}, J=8.2 \mathrm{~Hz}, 2 \mathrm{H} ; \mathrm{Ar}-\mathrm{H}), 7.40(\mathrm{~d}, J=8.2 \mathrm{~Hz}, 2 \mathrm{H} ; \mathrm{Ar}-\mathrm{H}), 6.34(\mathrm{~d}, J=15.8 \mathrm{~Hz}, 1 \mathrm{H}$; olefin-H), $6.18(\mathrm{dt}, J=15.8,6.3 \mathrm{~Hz}, 1 \mathrm{H}$; olefin-H), $2.21(\mathrm{q}, J=7.0 \mathrm{~Hz}, 2 \mathrm{H}), 1.50-1.42(\mathrm{~m}$, $2 \mathrm{H}), 1.30-1.24(\mathrm{~m}, 12 \mathrm{H}), 0.87(\mathrm{t}, J=6.8 \mathrm{~Hz}, 3 \mathrm{H}) ;{ }^{13} \mathrm{C} \mathrm{NMR}\left(100 \mathrm{MHz}, \mathrm{CDCl}_{3}\right) \delta 141.4(\mathrm{C})$, $134.1(\mathrm{CH}), 128.7\left(\mathrm{C}, \mathrm{q},{ }^{2} \mathrm{~J}_{\mathrm{C}-\mathrm{F}}=32.3 \mathrm{~Hz}, \mathrm{C}^{\prime} 4^{\prime}\right), 128.5(\mathrm{CH}), 126.0(\mathrm{CH} \times 2), 125.4(\mathrm{CH} \times 2$, $\left.\mathrm{q}^{3}{ }^{3} \mathrm{C}-F_{\mathrm{F}}=3.9 \mathrm{~Hz}, \mathrm{C}-3^{\prime}, \mathrm{C}-5^{\prime}\right), 124.4\left(\mathrm{C}, \mathrm{q}^{1}{ }^{1} \mathrm{~J}_{\mathrm{C}-F}=272.2 \mathrm{~Hz}, \mathrm{CF}_{3}\right), 33.1\left(\mathrm{CH}_{2}\right), 31.9\left(\mathrm{CH}_{2}\right), 29.6$ $\left(\mathrm{CH}_{2}\right), 29.5\left(\mathrm{CH}_{2}\right), 29.3\left(\mathrm{CH}_{2}\right), 29.2\left(\mathrm{CH}_{2}\right), 29.1\left(\mathrm{CH}_{2}\right), 22.7\left(\mathrm{CH}_{2}\right), 14.1\left(\mathrm{CH}_{3}\right) ; \mathrm{HRMS}(\mathrm{APCl})$ : $m / z$ calcd for $\left.\mathrm{C}_{18} \mathrm{H}_{24} \mathrm{~F}_{3}\left(\left[\mathrm{M}-\mathrm{H}_{2}+\mathrm{H}\right]\right]^{+}\right): 297.1825$, found 297.1809. (Z)-3b: ${ }^{1} \mathrm{H}$ NMR (400 $\left.\mathrm{MHz}^{\mathrm{CDCl}} \mathrm{CD}_{3}\right) \delta 7.56(\mathrm{~d}, J=8.2 \mathrm{~Hz}, 2 \mathrm{H} ; \mathrm{Ar}-\mathrm{H}), 7.34(\mathrm{~d}, J=8.2 \mathrm{~Hz}, 2 \mathrm{H} ; \mathrm{Ar}-\mathrm{H}), 6.40(\mathrm{~d}, J=$ 11.6 Hz, $1 \mathrm{H}$; olefin-H), $5.76(\mathrm{dt}, J=11.6,7.4 \mathrm{~Hz}, 1 \mathrm{H}$; olefin-H), 2.28 (qd, $J=7.6,1.5 \mathrm{~Hz}$, $2 \mathrm{H}), 1.48-1.40(\mathrm{~m}, 2 \mathrm{H}), 1.30-1.25(\mathrm{~m}, 12 \mathrm{H}), 0.85(\mathrm{t}, \mathrm{J}=6.8 \mathrm{~Hz}, 3 \mathrm{H}) ;{ }^{13} \mathrm{C} N M R(100 \mathrm{MHz}$, $\left.\mathrm{CDCl}_{3}\right) \delta 141.4(\mathrm{C}), 135.4(\mathrm{CH}), 128.9(\mathrm{CH} \times 2), 128.7\left(\mathrm{C}, \mathrm{q},{ }^{2} \mathrm{~J}_{\mathrm{C}-\mathrm{F}}=32.3 \mathrm{~Hz}, \mathrm{C}-4^{\prime}\right), 127.5$ (CH), $125.0\left(\mathrm{CH} \times 2, \mathrm{q},{ }^{3} J_{\mathrm{C}-F}=3.9 \mathrm{~Hz}, \mathrm{C}-3^{\prime}, \mathrm{C}^{-} 5^{\prime}\right), 124.2\left(\mathrm{C}, \mathrm{q},{ }^{1} \mathrm{~J}_{\mathrm{C}-\mathrm{F}}=272.2 \mathrm{~Hz}, \mathrm{CF}_{3}\right), 33.1$ $\left(\mathrm{CH}_{2}\right), 31.9\left(\mathrm{CH}_{2}\right), 29.8\left(\mathrm{CH}_{2}\right), 29.6\left(\mathrm{CH}_{2}\right), 29.5\left(\mathrm{CH}_{2}\right), 29.3\left(\mathrm{CH}_{2}\right), 28.6\left(\mathrm{CH}_{2}\right), 22.7\left(\mathrm{CH}_{2}\right)$, 14.1 $\left(\mathrm{CH}_{3}\right)$; HRMS (APCI): $\mathrm{m} / z$ calcd for $\mathrm{C}_{18} \mathrm{H}_{24} \mathrm{~F}_{3}\left(\left[\mathrm{M}-\mathrm{H}_{2}+\mathrm{H}\right]^{+}\right): 297.1825$, found 


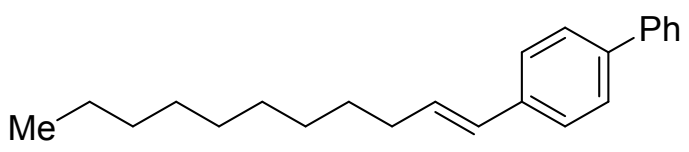

$(E)-3 e$

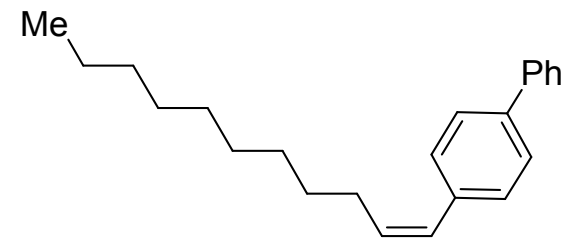

$(Z)-3 e$

$(E)$ - and (Z)-1-Phenyl-4-(undec-1-enyl)benzene (3e). Compounds $(E)$ - and (Z)-3e were synthesized from 1-methoxyundec-1-ene $1 \mathrm{a}(E / Z=55: 45,0.272 \mathrm{mmol}, 50 \mathrm{mg})$ and 2(biphenyl-4-yl)-5,5-dimethyl-1,3,2-dioxaborinane 2 e $(0.326 \mathrm{mmol}, 87 \mathrm{mg}$ ) according to general procedure. Standard work-up afforded crude $3 \mathbf{e}$ as a mixture of $(E)$ - and (Z)3e in a ratio $E / Z=97: 3$, and then purification by column chromatography on silica gel (eluent, petroleum ether/toluene $=20: 1$ ) yielded the title compound $3 \mathbf{e}$ as a white solid (72 $\mathrm{mg}, 0.235 \mathrm{mmol}, 86 \%$ yield). Spectral data are only given for the major stereoisomer $(E)-\mathbf{3 e}$, which was isolated as a pure compound. $(E)-3 \mathbf{e}:{ }^{1} \mathrm{H}$ NMR (400 $\left.\mathrm{MHz}, \mathrm{CDCl}_{3}\right) \delta 7.58(\mathrm{~d}, J=7.2 \mathrm{~Hz}, 2 \mathrm{H} ; \mathrm{Ar}-\mathrm{H}), 7.52(\mathrm{~d}, J=8.3 \mathrm{~Hz}, 2 \mathrm{H} ; \mathrm{Ar}-\mathrm{H}), 7.43-7.39(\mathrm{~m}$, 4H; Ar-H), $7.31(\mathrm{t}, J=7.4 \mathrm{~Hz}, 1 \mathrm{H} ; \mathrm{Ar}-\mathrm{H}), 6.40(\mathrm{~d}, J=15.9 \mathrm{~Hz}, 1 \mathrm{H}$; olefin-H), $6.26(\mathrm{dt}, J=$ 15.9, $6.8 \mathrm{~Hz}, 1 \mathrm{H}$; olefin-H), $2.22(\mathrm{q}, J=7.0 \mathrm{~Hz}, 2 \mathrm{H}), 1.48-1.42(\mathrm{~m}, 2 \mathrm{H}), 1.35-1.26(\mathrm{~m}$, 12H), $0.87(\mathrm{t}, J=7.2 \mathrm{~Hz}, 3 \mathrm{H}) ;{ }^{13} \mathrm{C}$ NMR $\left(100 \mathrm{MHz}, \mathrm{CDCl}_{3}\right) \delta 140.9(\mathrm{C}), 139.5(\mathrm{C}), 137.0$ (C), $131.5(\mathrm{CH}), 129.2(\mathrm{CH}), 128.7(\mathrm{CH} \times 2), 127.2(\mathrm{CH}), 127.1(\mathrm{CH} \times 2), 126.9(\mathrm{CH} \times 2)$, 126.3 $(\mathrm{CH} \times 2), 33.1\left(\mathrm{CH}_{2}\right), 31.9\left(\mathrm{CH}_{2}\right), 29.6\left(\mathrm{CH}_{2}\right), 29.5\left(\mathrm{CH}_{2}\right), 29.4\left(\mathrm{CH}_{2}\right) 29.3\left(\mathrm{CH}_{2}\right), 29.2$ $\left(\mathrm{CH}_{2}\right), 22.7\left(\mathrm{CH}_{2}\right), 14.1\left(\mathrm{CH}_{3}\right)$; HRMS $(\mathrm{APCl}): \mathrm{m} / z$ calcd for $\mathrm{C}_{23} \mathrm{H}_{31}\left([\mathrm{M}+\mathrm{H}]^{+}\right)$: 307.2420, found 307.2417 . 


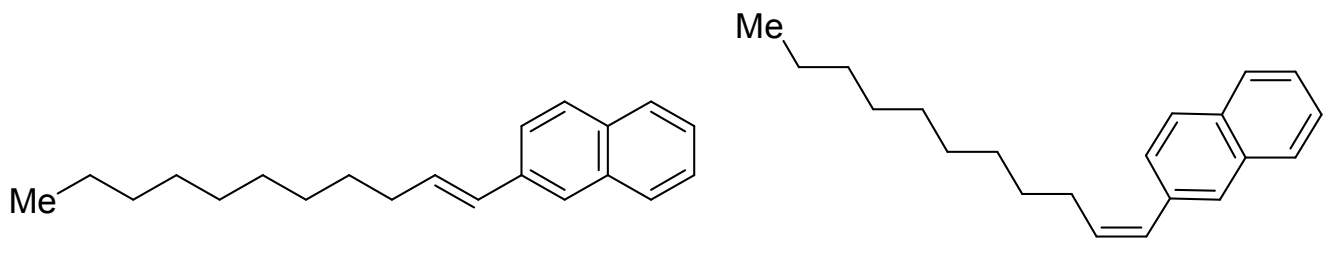

$(E)-3 \mathbf{f}$

(Z)-3f

$(E)$ - and (Z)-2-(Undec-1-enyl)naphthalene (3f). Compounds $(E)$ - and (Z)-3f were synthesized from 1-methoxyundec-1-ene $1 \mathrm{a}(E / Z=55: 45,0.272 \mathrm{mmol}, 50 \mathrm{mg})$ and 5,5dimethyl-2-(naphthalen-2-yl)-1,3,2-dioxaborinane 2 f (0.326 mmol, $78 \mathrm{mg}$ ) according to general procedure. Standard work-up afforded crude $\mathbf{3 f}$ as a mixture of $(E)$ - and $(Z)$ 3f in a ratio $E / Z=97: 3$, and then purification by column chromatography on silica gel (eluent, petroleum ether/toluene $=20: 1$ ) yielded the title compound $\mathbf{3 f}$ as a white solid (63 mg, $0.225 \mathrm{mmol}, 83 \%$ yield). Spectral data are only given for the major stereoisomer (E)-3f, which was isolated as a pure compound. (E)-3f: ${ }^{1} \mathrm{H} N \mathrm{NMR}(400 \mathrm{MHz}$, $\left.\mathrm{CDCl}_{3}\right) \delta 7.76(\mathrm{t}, J=8.6 \mathrm{~Hz}, 3 \mathrm{H} ; \mathrm{Ar}-\mathrm{H}), 7.66(\mathrm{~s}, 1 \mathrm{H} ; \mathrm{Ar}-\mathrm{H}), 7.57(\mathrm{dd}, J=8.5,1.6 \mathrm{~Hz}, 1 \mathrm{H}$; $\operatorname{Ar}-\mathrm{H}), 7.45-7.37(\mathrm{~m}, 2 \mathrm{H} ; \mathrm{Ar}-\mathrm{H}), 6.52(\mathrm{~d}, J=15.8 \mathrm{~Hz}, 1 \mathrm{H}$; olefin-H), $6.35(\mathrm{dt}, J=15.8,6.9$ $\mathrm{Hz}, 1 \mathrm{H}$; olefin-H), $2.23(\mathrm{q}, J=7.8 \mathrm{~Hz}, 2 \mathrm{H}), 1.49-1.45(\mathrm{~m}, 2 \mathrm{H}), 1.37-1.32(\mathrm{~m}, 12 \mathrm{H}), 0.87$ $(\mathrm{t}, J=7.2 \mathrm{~Hz}, 3 \mathrm{H}) ;{ }^{13} \mathrm{C} \mathrm{NMR}\left(100 \mathrm{MHz}, \mathrm{CDCl}_{3}\right) \delta 135.4(\mathrm{C}), 133.7$ (C), $132.6(\mathrm{C}), 131.8$ (CH), $129.8(\mathrm{CH}), 128.0(\mathrm{CH}), 127.8(\mathrm{CH}), 127.6(\mathrm{CH}), 126.1(\mathrm{CH}), 125.4(\mathrm{CH}), 125.2(\mathrm{CH})$, $123.6(\mathrm{CH}), 33.2\left(\mathrm{CH}_{2}\right), 31.9\left(\mathrm{CH}_{2}\right), 29.6\left(\mathrm{CH}_{2} \times 2\right), 29.4\left(\mathrm{CH}_{2}\right) 29.3\left(\mathrm{CH}_{2} \times 2\right), 22.7\left(\mathrm{CH}_{2}\right)$, 14.1 $\left(\mathrm{CH}_{3}\right)$; HRMS (APCI): $m / z$ calcd for $\mathrm{C}_{21} \mathrm{H}_{29}\left([\mathrm{M}+\mathrm{H}]^{+}\right): 281.2224$, found 281.2245.<smiles>CCCCCCCCC/C=C/c1cccc2ccccc12</smiles>

$(E)-3 g$

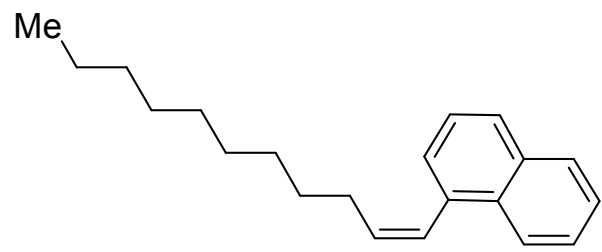

(Z)-3g

$(E)$ - and (Z)-1-(Undec-1-enyl)naphthalene (3g). Compounds $(E)$ - and (Z)-3g were synthesized from 1-methoxyundec-1-ene $1 \mathrm{a}(E / Z=55: 45,0.272 \mathrm{mmol}, 50 \mathrm{mg})$ and 5,5- 
dimethyl-2-(naphthalen-4-yl)-1,3,2-dioxaborinane $\mathbf{2 g}(0.326 \mathrm{mmol}, 78 \mathrm{mg})$ according to general procedure. Standard work-up afforded crude $\mathbf{3 g}$ as a mixture of $(E)$ - and (Z)$3 \mathrm{~g}$ in a ratio $E / Z=89: 11$, and then purification by column chromatography on silica gel (eluent, petroleum ether/toluene $=20: 1$ ) yielded the title compound $\mathbf{3 g}$ as a paleyellow oil (61 mg, $0.218 \mathrm{mmol}, 80 \%$ yield). The $(E)$ - and (Z)-isomers were partially separated by column chromatography. $(E)-3 \mathrm{~g}:{ }^{1} \mathrm{H} \mathrm{NMR}\left(400 \mathrm{MHz}, \mathrm{CDCl}_{3}\right) \delta 8.11$ (dd, $J$ $=7.7,3.3 \mathrm{~Hz}, 1 \mathrm{H} ; \mathrm{Ar}-\mathrm{H}), 7.82(\mathrm{dd}, J=6.9,2.4 \mathrm{~Hz}, 1 \mathrm{H} ; \mathrm{Ar}-\mathrm{H}), 7.72(\mathrm{~d}, J=8.4 \mathrm{~Hz}, 1 \mathrm{H} ; \operatorname{Ar}-$ H), $7.54(\mathrm{~d}, J=7.1 \mathrm{~Hz}, 1 \mathrm{H} ; \operatorname{Ar}-\mathrm{H}), 7.52-7.43(\mathrm{~m}, 2 \mathrm{H} ; \operatorname{Ar}-\mathrm{H}), 7.42$ (d, J = 7.9 Hz, 1H; Ar-H), $7.09(\mathrm{~d}, J=15.5 \mathrm{~Hz}, 1 \mathrm{H}$; olefin-H), $6.22(\mathrm{dt}, J=15.5,6.9 \mathrm{~Hz}, 1 \mathrm{H}$; olefin-H), $2.32(\mathrm{qd}, J=$ 7.2, $1.5 \mathrm{~Hz}, 2 \mathrm{H}), 1.56-1.49(\mathrm{~m}, 2 \mathrm{H}), 1.40-1.23(\mathrm{~m}, 12 \mathrm{H}), 0.88(\mathrm{t}, \mathrm{J}=7.2 \mathrm{~Hz}, 3 \mathrm{H}) ;{ }^{13} \mathrm{C}$ NMR $\left(100 \mathrm{MHz}, \mathrm{CDCl}_{3}\right) \delta 135.8(\mathrm{C}), 134.6(\mathrm{C}), 133.6(\mathrm{CH}), 131.2(\mathrm{C}), 128.5(\mathrm{CH}), 127.2(\mathrm{CH})$, $126.9(\mathrm{CH}), 125.8(\mathrm{CH}), 125.7(\mathrm{CH}), 125.6(\mathrm{CH}), 124.0(\mathrm{CH}), 123.5(\mathrm{CH}), 33.5\left(\mathrm{CH}_{2}\right), 32.0$ $\left(\mathrm{CH}_{2}\right), 29.7\left(\mathrm{CH}_{2}\right), 29.6\left(\mathrm{CH}_{2}\right), 29.4\left(\mathrm{CH}_{2}\right) 29.3\left(\mathrm{CH}_{2} \times 2\right), 22.7\left(\mathrm{CH}_{2}\right), 14.2\left(\mathrm{CH}_{3}\right)$; HRMS (APCI): $m / z$ calcd for $\mathrm{C}_{21} \mathrm{H}_{29}\left([\mathrm{M}+\mathrm{H}]^{+}\right): 281.2264$, found 281.2269. (Z)-3g: ${ }^{1} \mathrm{H}$ NMR (400 $\left.\mathrm{MHz}, \mathrm{CDCl}_{3}\right) \delta 8.00-7.97(\mathrm{~m}, 1 \mathrm{H} ; \mathrm{Ar}-\mathrm{H}), 7.84-7.82(\mathrm{~m}, 1 \mathrm{H} ; \mathrm{Ar}-\mathrm{H}), 7.74(\mathrm{~d}, J=8.1 \mathrm{~Hz}, 1 \mathrm{H}$; Ar-H), 7.48-7.45 (m, 2H; Ar-H), $7.43(\mathrm{~d}, J=8.1 \mathrm{~Hz}, 1 \mathrm{H} ; \operatorname{Ar}-\mathrm{H}), 7.31(\mathrm{~d}, J=7.1 \mathrm{~Hz}, 1 \mathrm{H} ; \mathrm{Ar}-$ H), $6.85(\mathrm{~d}, J=11.4 \mathrm{~Hz}, 1 \mathrm{H}$; olefin-H), $5.91(\mathrm{dt}, J=11.4,7.4 \mathrm{~Hz}, 1 \mathrm{H}$; olefin-H), 2.11 (qd, $J=7.4,1.6 \mathrm{~Hz}, 2 \mathrm{H}), 1.41-1.33(\mathrm{~m}, 2 \mathrm{H}), 1.26-1.17(\mathrm{~m}, 12 \mathrm{H}), 0.86(\mathrm{t}, J=7.3 \mathrm{~Hz}, 3 \mathrm{H}) ;{ }^{13} \mathrm{C}$ NMR (100 MHz, $\left.\mathrm{CDCl}_{3}\right) \delta 135.0(\mathrm{C}), 134.7(\mathrm{CH}), 133.5(\mathrm{C}), 132.0(\mathrm{C}), 128.3(\mathrm{CH}), 127.1$ (CH), $126.8(\mathrm{CH}), 126.4(\mathrm{CH}), 125.7(\mathrm{CH}), 125.6(\mathrm{CH}), 125.3(\mathrm{CH}), 125.1(\mathrm{CH}), 31.9\left(\mathrm{CH}_{2}\right)$, $29.8\left(\mathrm{CH}_{2}\right), 29.7\left(\mathrm{CH}_{2}\right), 29.6\left(\mathrm{CH}_{2}\right) 29.5\left(\mathrm{CH}_{2}\right), 29.3\left(\mathrm{CH}_{2}\right), 28.7\left(\mathrm{CH}_{2}\right), 22.7\left(\mathrm{CH}_{2}\right), 14.1$ $\left(\mathrm{CH}_{3}\right) ; \operatorname{HRMS}(\mathrm{APCl}): \mathrm{m} / \mathrm{z}$ calcd for $\mathrm{C}_{21} \mathrm{H}_{29}\left([\mathrm{M}+\mathrm{H}]^{+}\right):$: 281.2264, found 281.2269. 


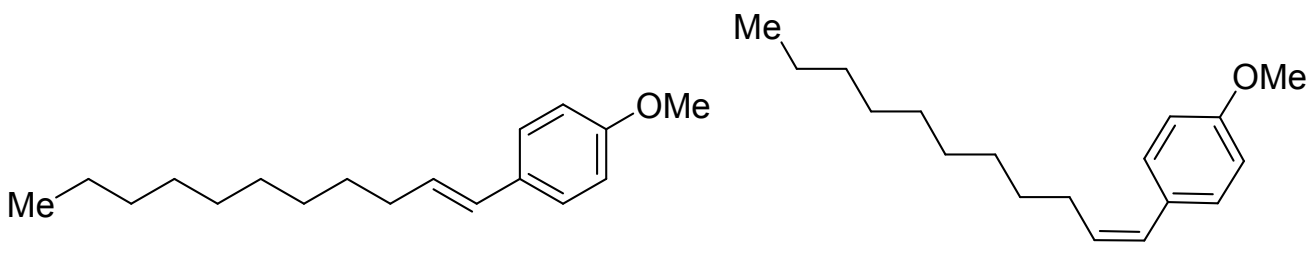

$(E)-3 h$

$(Z)-3 h$

(E)- and (Z)-1-Methoxy-4-(undec-1-enyl)benzene (3h). Compounds (E)- and (Z)-3h were synthesized from 1-methoxyundec-1-ene 1a $(E / Z=55: 45,0.272 \mathrm{mmol}, 50 \mathrm{mg})$ and 2-(4-methoxyphenyl)-5,5-dimethyl-1,3,2-dioxaborinane $\mathbf{2 h}(0.326 \mathrm{mmol}, 72 \mathrm{mg})$ according to general procedure. Standard work-up afforded crude $3 \mathrm{~h}$ as a mixture of $(E)-$ and $(Z)-3 \mathrm{~h}$ in a ratio $E / Z=96: 4$, and then purification by column chromatography on silica gel (eluent, petroleum ether/toluene $=10: 1$ ) yielded the title compound $3 \mathrm{~h}$ as a clear oil (66 mg, $0.254 \mathrm{mmol}, 93 \%$ yield). The $(E)$ - and (Z)-isomers were partially separated by column chromatography. (E)-3h: ${ }^{1} \mathrm{H} \mathrm{NMR}\left(400 \mathrm{MHz}, \mathrm{CDCl}_{3}\right) \delta 7.26(\mathrm{~d}, J=$ $9.1 \mathrm{~Hz}, 2 \mathrm{H}, \mathrm{Ar}-\mathrm{H}), 6.81(\mathrm{~d}, J=8.7 \mathrm{~Hz}, 2 \mathrm{H}, \mathrm{Ar}-\mathrm{H}), 6.30(\mathrm{~d}, J=15.8 \mathrm{~Hz}, 1 \mathrm{H}$; olefin-H), 6.06 $(\mathrm{dt}, J=15.8,6.9 \mathrm{~Hz}, 1 \mathrm{H}), 3.78(\mathrm{~s}, 3 \mathrm{H} ; \mathrm{OMe}), 2.16(\mathrm{qd}, J=6.9,1.2 \mathrm{~Hz}, 2 \mathrm{H}), 1.46-1.39(\mathrm{~m}$, $2 \mathrm{H}), 1.30-1.25(\mathrm{~m}, 12 \mathrm{H}), 0.86(\mathrm{t}, J=7.2 \mathrm{~Hz}, 3 \mathrm{H}) ;{ }^{13} \mathrm{C} \mathrm{NMR}\left(100 \mathrm{MHz}, \mathrm{CDCl}_{3}\right) \delta 158.6(\mathrm{C})$, $130.8(\mathrm{C}), 129.1(\mathrm{CH}), 129.0(\mathrm{CH}), 126.9(\mathrm{CH} \times 2), 113.9(\mathrm{CH} \times 2), 55.3\left(\mathrm{CH}_{3}\right), 33.0\left(\mathrm{CH}_{2}\right)$, $31.9\left(\mathrm{CH}_{2}\right), 29.6\left(\mathrm{CH}_{2}\right), 29.5\left(\mathrm{CH}_{2} \times 2\right), 29.3\left(\mathrm{CH}_{2}\right) 29.2\left(\mathrm{CH}_{2}\right), 22.7\left(\mathrm{CH}_{2}\right), 14.1\left(\mathrm{CH}_{3}\right)$; HRMS (APCI): $m / z$ calcd for $\mathrm{C}_{18} \mathrm{H}_{29} \mathrm{O}\left([\mathrm{M}+\mathrm{H}]^{+}\right): 261.2213$, found 261.2216. (Z)-3h: ${ }^{1} \mathrm{H}$ $\operatorname{NMR}\left(400 \mathrm{MHz}, \mathrm{CDCl}_{3}\right) \delta 7.20(\mathrm{~d}, J=8.7 \mathrm{~Hz}, 2 \mathrm{H}, \mathrm{Ar}-\mathrm{H}), 6.85(\mathrm{~d}, J=8.8 \mathrm{~Hz}, 2 \mathrm{H}, \mathrm{Ar}-\mathrm{H})$, $6.31(\mathrm{~d}, J=11.6 \mathrm{~Hz}, 1 \mathrm{H}$; olefin-H), $5.55(\mathrm{dt}, J=11.6,7.2 \mathrm{~Hz}, 1 \mathrm{H}), 3.79(\mathrm{~s}, 3 \mathrm{H} ; \mathrm{OMe}), 2.30$ ( $q d, J=7.4,1.8 \mathrm{~Hz}, 2 \mathrm{H}), 1.45-1.38(\mathrm{~m}, 2 \mathrm{H}), 1.29-1.23(\mathrm{~m}, 12 \mathrm{H}), 0.86(\mathrm{t}, J=7.2 \mathrm{~Hz}, 3 \mathrm{H})$; ${ }^{13} \mathrm{C} \mathrm{NMR}(100 \mathrm{MHz}, \mathrm{CDCl})$ ) $\delta 158.1(\mathrm{C}), 131.7(\mathrm{CH}), 130.5(\mathrm{C}), 129.9(\mathrm{CH} \times 2), 128.0(\mathrm{CH})$, $113.5(\mathrm{CH} \times 2), 55.2\left(\mathrm{CH}_{3}\right), 31.9\left(\mathrm{CH}_{2}\right), 30.0\left(\mathrm{CH}_{2}\right), 29.6\left(\mathrm{CH}_{2}\right), 29.5\left(\mathrm{CH}_{2}\right), 29.4\left(\mathrm{CH}_{2}\right)$, $29.3\left(\mathrm{CH}_{2}\right), 28.6\left(\mathrm{CH}_{2}\right), 22.7\left(\mathrm{CH}_{2}\right), 14.1\left(\mathrm{CH}_{3}\right)$; HRMS (APCI): $\mathrm{m} / \mathrm{z}$ calcd for $\mathrm{C}_{18} \mathrm{H}_{29} \mathrm{O}([\mathrm{M}$ $\left.+\mathrm{H}^{+}\right):$:261.2213, found 261.2216. 


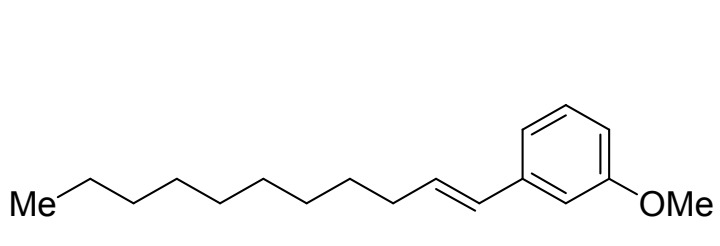

$(E)-\mathbf{3 i}$

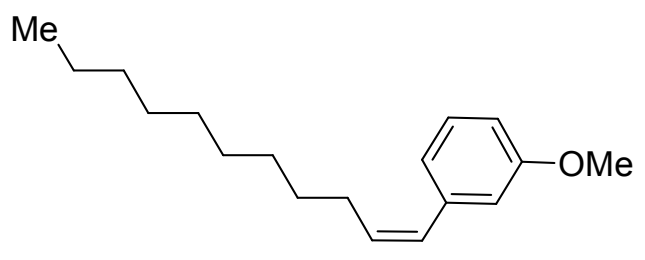

$(Z)-\mathbf{3 i}$

$(E)$ - and (Z)-1-Methoxy-3-(undec-1-enyl)benzene (3i). Compounds $(E)$ - and (Z)-3i were synthesized from 1-methoxyundec-1-ene $1 \mathrm{a}(E / Z=55: 45,0.272 \mathrm{mmol}, 50 \mathrm{mg})$ and 2(3-methoxyphenyl)-5,5-dimethyl-1,3,2-dioxaborinane $\mathbf{2 i}(0.326 \mathrm{mmol}, 72 \mathrm{mg}$ ) according to general procedure. Standard work-up afforded crude $\mathbf{3 i}$ as a mixture of $(E)$ - and $(Z)-3 \mathbf{i}$ in a ratio $E / Z=97: 3$, and then purification by column chromatography on silica gel (eluent, petroleum ether/toluene $=10: 1$ ) yielded the title compound $\mathbf{3 i}$ as a clear oil (64 mg, $0.246 \mathrm{mmol}, 90 \%$ yield). Spectral data are only given for the major stereoisomer (E)-3i, which was isolated as a pure compound. $(E)-3 \mathbf{i}:{ }^{1} \mathrm{H} N M R(400 \mathrm{MHz}$, $\left.\mathrm{CDCl}_{3}\right) \delta 7.19(\mathrm{t}, J=7.9 \mathrm{~Hz}, 1 \mathrm{H}, \mathrm{Ar}-\mathrm{H}), 6.92(\mathrm{dd}, J=7.7 \mathrm{~Hz}, 1 \mathrm{H}, \mathrm{Ar}-\mathrm{H}), 7.87(\mathrm{~s}, 1 \mathrm{H}, \mathrm{Ar}-\mathrm{H})$, $6.73(\mathrm{dd}, J=8.3,2.4 \mathrm{~Hz}, 1 \mathrm{H}, \mathrm{Ar}-\mathrm{H}), 6.33(\mathrm{~d}, J=15.8 \mathrm{~Hz}, 1 \mathrm{H}$, olefin- $\mathrm{H}), 6.21(\mathrm{dt}, J=15.8$, $6.7 \mathrm{~Hz}, 1 \mathrm{H}$, olefin-H), $3.79(\mathrm{~s}, 3 \mathrm{H}, \mathrm{OMe}), 2.18(\mathrm{q}, J=7.1 \mathrm{~Hz}, 2 \mathrm{H}), 1.46-1.41(\mathrm{~m}, 2 \mathrm{H})$, 1.29-1.26 (m, 12H), $0.87(\mathrm{t}, J=7.2 \mathrm{~Hz}, 3 \mathrm{H}) ;{ }^{13} \mathrm{C} \mathrm{NMR}\left(100 \mathrm{MHz}, \mathrm{CDCl}_{3}\right) \delta 159.8(\mathrm{C})$, $139.4(\mathrm{C}), 131.6(\mathrm{CH}), 129.5(\mathrm{C}), 129.4(\mathrm{CH}), 118.6(\mathrm{CH}), 112.4(\mathrm{CH}), 111.2(\mathrm{CH}), 55.2$ $\left(\mathrm{CH}_{3}\right), 33.0\left(\mathrm{CH}_{2}\right), 31.9\left(\mathrm{CH}_{2}\right), 29.6\left(\mathrm{CH}_{2}\right), 29.5\left(\mathrm{CH}_{2}\right), 29.3\left(\mathrm{CH}_{2} \times 2\right), 29.2\left(\mathrm{CH}_{2}\right), 22.7$ $\left(\mathrm{CH}_{2}\right), 14.1\left(\mathrm{CH}_{3}\right)$; HRMS (APCI): $m / z$ calcd for $\mathrm{C}_{18} \mathrm{H}_{29} \mathrm{O}\left([\mathrm{M}+\mathrm{H}]^{+}\right): 261.2213$, found 261.2207.

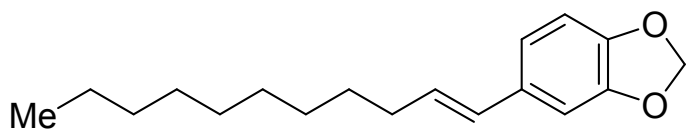

$(E)-\mathbf{3}$

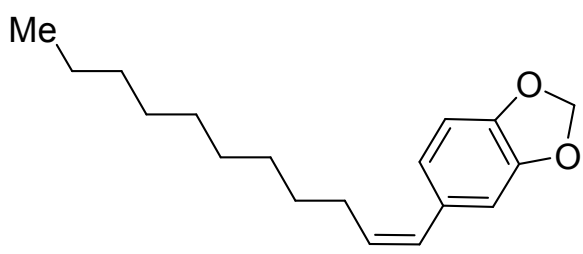

$(Z)-3 \mathbf{j}$

$(E)$ - and (Z)-5-(Undec-1-enyl)benzo[d][1,3]dioxole (3j). Compounds $(E)$ - and $(Z)-3 \mathbf{j}$ 
were synthesized from 1-methoxyundec-1-ene $1 \mathrm{a}(E / Z=55: 45,0.272 \mathrm{mmol}, 50 \mathrm{mg})$ and 2-(benzo[d][1,3]dioxol-5-yl)-5,5-dimethyl-1,3,2-dioxaborinane $2 \mathbf{j}(0.326 \mathrm{mmol}, 76$ mg) according to general procedure. Standard work-up afforded crude $\mathbf{3} \mathbf{j}$ as a mixture of $(E)$ - and $(Z)-3 \mathbf{j}$ in a ratio $E / Z=97: 3$, and then purification by column chromatography on silica gel (eluent, petroleum ether/toluene $=10: 1$ ) yielded the title compound $\mathbf{3} \mathbf{j}$ as a clear oil $(60 \mathrm{mg}, 0.219 \mathrm{mmol}, 81 \%$ yield). Spectral data are only given for the major stereoisomer $(E)-\mathbf{3} \mathbf{j}$, which was isolated as a pure compound. $(E)-\mathbf{3} \mathbf{j}:{ }^{1} \mathrm{H} N \mathrm{NMR}(400 \mathrm{MHz}$, $\left.\mathrm{CDCl}_{3}\right) \delta 6.88(\mathrm{~d}, J=1.3 \mathrm{~Hz}, 1 \mathrm{H} ; \mathrm{Ar}-\mathrm{H}), 6.75-6.70(\mathrm{~m}, 2 \mathrm{H} ; \mathrm{Ar}-\mathrm{H}), 6.26(\mathrm{~d}, J=15.8 \mathrm{~Hz}, 1 \mathrm{H}$; olefin-H), $6.03(\mathrm{dt}, J=15.7,6.9 \mathrm{~Hz}, 1 \mathrm{H}$; olefin-H), $5.91(\mathrm{~s}, 2 \mathrm{H}), 2.16(\mathrm{qd}, J=7.2,1.3 \mathrm{~Hz}$, $2 \mathrm{H}), 1.44-1.39(\mathrm{~m}, 2 \mathrm{H}), 1.28-1.25(\mathrm{~m}, 12 \mathrm{H}), 0.87(\mathrm{t}, J=7.2 \mathrm{~Hz}, 3 \mathrm{H}) ;{ }^{13} \mathrm{C}$ NMR $(100 \mathrm{MHz}$, $\left.\mathrm{CDCl}_{3}\right) \delta 147.9(\mathrm{C}), 146.5(\mathrm{C}), 132.5(\mathrm{CH}), 129.5(\mathrm{CH}), 129.2(\mathrm{CH}), 120.1(\mathrm{CH}), 108.2(\mathrm{CH})$, $105.4(\mathrm{CH}), 100.9\left(\mathrm{CH}_{2}\right), 32.9\left(\mathrm{CH}_{2}\right), 31.9\left(\mathrm{CH}_{2}\right), 29.6\left(\mathrm{CH}_{2}\right), 29.5\left(\mathrm{CH}_{2} \times 2\right), 29.3\left(\mathrm{CH}_{2}\right)$, $29.2\left(\mathrm{CH}_{2}\right), 22.7\left(\mathrm{CH}_{2}\right), 14.1\left(\mathrm{CH}_{3}\right)$; HRMS (APCl): $\mathrm{m} / z$ calcd for $\mathrm{C}_{18} \mathrm{H}_{27} \mathrm{O}_{2}\left([\mathrm{M}+\mathrm{H}]^{+}\right)$: 275.2006, found 275.1990 .

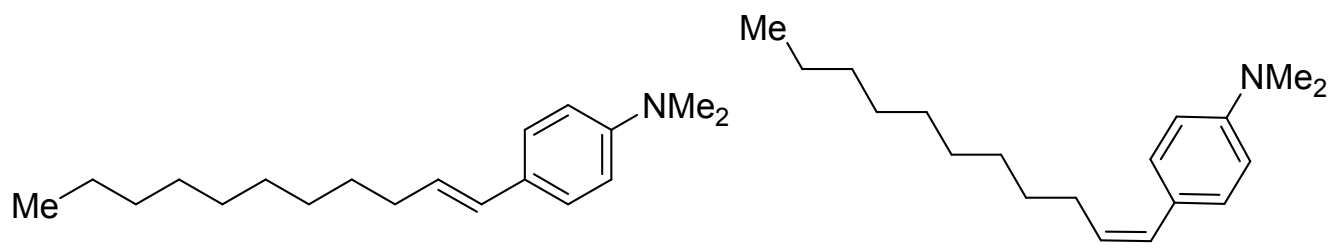

$(E)-3 \mathbf{k}$

$(Z)-3 k$

$(E)$ - and (Z)-N,N-Dimethyl-4-(undec-1-enyl)benzenamine (3k). Compounds (E)- and (Z)-3k were synthesized from 1-methoxyundec-1-ene 1a $(E / Z=55: 45,0.272 \mathrm{mmol}, 50$ $\mathrm{mg}$ ) and $\mathrm{N}, \mathrm{N}$-dimethyl-4-(5,5-dimethyl-1,3,2-dioxaborinan-2-yl)benzenamine $\mathbf{2 k}$ (0.326 mmol, $76 \mathrm{mg}$ ) according to general procedure. Standard work-up afforded crude $\mathbf{3 k}$ as a mixture of $(E)$ - and $(Z)-\mathbf{3 k}$ in a ratio $E / Z=97: 3$, and then purification by column chromatography on silica gel (eluent, petroleum ether/ ethyl acetate $=7: 1$ ) yielded the title compound $\mathbf{3 k}$ as a pale-yellow oil (66 mg, $0.242 \mathrm{mmol}, 89 \%$ yield). 
Spectral data are only given for the major stereoisomer $(E)-\mathbf{3 k}$, which was isolated as a pure compound. (E)-3k: ${ }^{1} \mathrm{H}$ NMR $\left(400 \mathrm{MHz}, \mathrm{CDCl}_{3}\right) \delta 7.22(\mathrm{~d}, J=8.7 \mathrm{~Hz}, 2 \mathrm{H} ; \mathrm{Ar}-\mathrm{H})$, $6.67(\mathrm{~d}, J=8.7 \mathrm{~Hz}, 2 \mathrm{H} ; \mathrm{Ar}-\mathrm{H}), 6.27(\mathrm{~d}, J=15.8 \mathrm{~Hz}, 1 \mathrm{H}$; olefin-H), 6.00 (dt, $J=15.8,6.9$ $\mathrm{Hz}, 1 \mathrm{H}$; olefin-H), $2.92(\mathrm{~s}, 6 \mathrm{H}), 2.15(\mathrm{q}, J=6.9 \mathrm{~Hz}, 2 \mathrm{H}), 1.44-1.39(\mathrm{~m}, 2 \mathrm{H}), 1.30-1.25(\mathrm{~m}$, $12 \mathrm{H}), 0.87(\mathrm{t}, J=6.8 \mathrm{~Hz}, 3 \mathrm{H}) ;{ }^{13} \mathrm{C}$ NMR $\left(100 \mathrm{MHz}, \mathrm{CDCl}_{3}\right) \delta 149.6(\mathrm{C}), 129.3(\mathrm{CH}), 127.2$ (C), $126.7(\mathrm{CH} \times 4), 112.8(\mathrm{CH}), 40.7\left(\mathrm{CH}_{3} \times 2\right), 33.1\left(\mathrm{CH}_{2}\right), 31.9\left(\mathrm{CH}_{2}\right), 29.7\left(\mathrm{CH}_{2}\right), 29.6$ $\left(\mathrm{CH}_{2} \times 2\right), 29.3\left(\mathrm{CH}_{2}\right), 29.2\left(\mathrm{CH}_{2}\right), 22.7\left(\mathrm{CH}_{2}\right), 14.1\left(\mathrm{CH}_{3}\right) ; \mathrm{HRMS}(\mathrm{APCl}): \mathrm{m} / \mathrm{z}$ calcd for $\mathrm{C}_{19} \mathrm{H}_{32} \mathrm{~N}\left([\mathrm{M}+\mathrm{H}]^{+}\right): 274.2529$, found 274.2531.

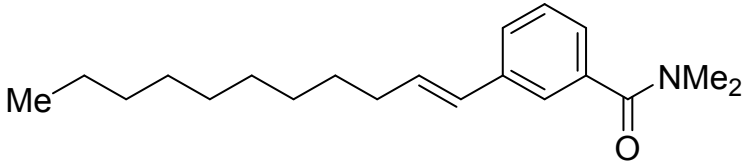

$(E)-3 \mid$

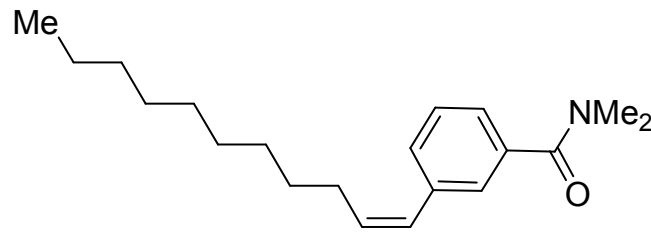

(Z)-31

$(E)$ - and (Z)-N,N-Dimethyl-3-(undec-1-enyl)benzamide (3I). Compounds (E)- and (Z)3I were synthesized from 1-methoxyundec-1-ene $1 \mathrm{a}(E / Z=55: 45,0.272 \mathrm{mmol}, 50 \mathrm{mg})$ and N,N-dimethyl-3-(5,5-dimethyl-1,3,2-dioxaborinan-2-yl)benzamide $2 \mathbf{l}(0.326 \mathrm{mmol}$, $85 \mathrm{mg}$ ) according to general procedure at $105^{\circ} \mathrm{C}$. Standard work-up afforded crude $\mathbf{3 l}$ as a mixture of $(E)$ - and $(Z)-3 \mid$ in a ratio $E / Z=95: 5$, and then purification by column chromatography on silica gel (eluent, petroleum ether/ethyl acetate $=3: 1$ to $1: 1$ ) yielded the title compound $3 \mathbf{l}$ as a pale-yellow oil (69 $\mathrm{mg}, 0.229 \mathrm{mmol}, 84 \%$ yield). Spectral data are only given for the major stereoisomer $(E)-\mathbf{3} \mathbf{I}$, which was isolated as a pure compound. $(E)-3 \mathrm{l}:{ }^{1} \mathrm{H}$ NMR $\left(400 \mathrm{MHz}, \mathrm{CDCl}_{3}\right) \delta 7.36(\mathrm{~s}, 1 \mathrm{H} ; \mathrm{Ar}-\mathrm{H}), 7.33(\mathrm{~d}, J=7.5$ $\mathrm{Hz}, 1 \mathrm{H} ; \operatorname{Ar}-\mathrm{H}), 7.28(\mathrm{t}, J=7.5 \mathrm{~Hz}, 1 \mathrm{H} ; \operatorname{Ar}-\mathrm{H}), 7.18(\mathrm{~d}, J=7.5 \mathrm{~Hz}, 1 \mathrm{H} ; \operatorname{Ar}-\mathrm{H}), 6.34(\mathrm{~d}, J=$ $15.9 \mathrm{~Hz}, 1 \mathrm{H}$; olefin-H), $6.23\left(\mathrm{dt}, \mathrm{J}=15.9,6.7 \mathrm{~Hz}, 1 \mathrm{H}\right.$; olefin-H), $3.08\left(\mathrm{~s}, 3 \mathrm{H} ; \mathrm{NCH}_{3}\right), 2.95$ $\left(\mathrm{s}, 3 \mathrm{H} ; \mathrm{NCH}_{3}\right), 2.18(\mathrm{q}, J=7.0 \mathrm{~Hz}, 2 \mathrm{H}), 1.47-1.40(\mathrm{~m}, 2 \mathrm{H}), 1.28-1.25(\mathrm{~m}, 12 \mathrm{H}), 0.86(\mathrm{t}, J$ $=7.2 \mathrm{~Hz}, 3 \mathrm{H}) ;{ }^{13} \mathrm{C}$ NMR $\left(100 \mathrm{MHz}, \mathrm{CDCl}_{3}\right) \delta 171.7(\mathrm{C}), 138.2(\mathrm{C}), 136.5(\mathrm{C}), 132.4(\mathrm{CH})$, 
$128.9(\mathrm{CH}), 128.4(\mathrm{CH}), 126.9(\mathrm{CH}), 125.1(\mathrm{CH}), 124.5(\mathrm{CH}), 39.5\left(\mathrm{CH}_{3}\right), 35.3\left(\mathrm{CH}_{3}\right), 33.0$ $\left(\mathrm{CH}_{2}\right), 31.9\left(\mathrm{CH}_{2}\right), 29.5\left(\mathrm{CH}_{2} \times 2\right), 29.3\left(\mathrm{CH}_{2} \times 2\right), 29.2\left(\mathrm{CH}_{2}\right), 22.6\left(\mathrm{CH}_{2}\right), 14.1\left(\mathrm{CH}_{3}\right) ; \mathrm{HRMS}$ (APCI): $m / z$ calcd for $\mathrm{C}_{20} \mathrm{H}_{32} \mathrm{NO}\left([\mathrm{M}+\mathrm{H}]^{+}\right): 302.2478$, found 302.2474 .

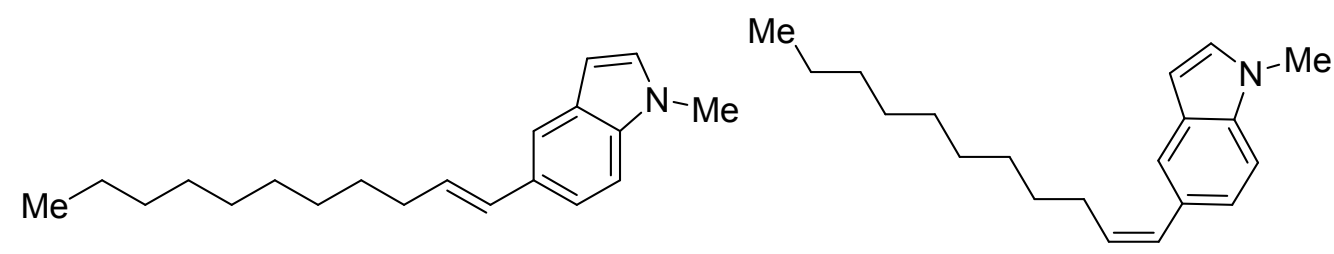

$(E)-3 m$

$(Z)-3 m$

(E)- and (Z)-1-Methyl-5-(undec-1-enyl)-1H-indole (3m). Compounds (E)- and (Z)-3m were synthesized from 1-methoxyundec-1-ene 1a $(E / Z=55: 45,0.272 \mathrm{mmol}, 50 \mathrm{mg})$ and 1-methyl-5-(5,5-dimethyl-1,3,2-dioxaborinan-2-yl)-1H-indole $2 \mathrm{~m}$ (0.326 mmol, 79 $\mathrm{mg}$ ) according to general procedure at $105^{\circ} \mathrm{C}$. Standard work-up afforded crude $\mathbf{3 m}$ as a mixture of $(E)$ - and $(Z)-3 \mathrm{~m}$ in a ratio $E / Z=95: 5$, and then purification by column chromatography on silica gel (eluent, petroleum ether/ether $=100: 1$ ) yielded the title compound $3 \mathbf{l}$ as a pale-yellow oil $(62 \mathrm{mg}, 0.219 \mathrm{mmol}, 80 \%$ yield). Spectral data are only given for the major stereoisomer $(E)-3 m$, which was isolated as a pure compound. (E)-3m: ${ }^{1} \mathrm{H}$ NMR (400 MHz, $\left.\mathrm{CDCl}_{3}\right) \delta 7.54(\mathrm{~s}, 1 \mathrm{H}, \mathrm{Ar}-\mathrm{H}), 7.28(\mathrm{dd}, J=8.5,1.0 \mathrm{~Hz}, 1 \mathrm{H}$, $\operatorname{Ar}-\mathrm{H}$ ), 7.22 (d, J= $8.5 \mathrm{~Hz}, 1 \mathrm{H}, \operatorname{Ar}-\mathrm{H}), 6.99(\mathrm{~d}, J=3.0 \mathrm{~Hz}, 1 \mathrm{H}, \operatorname{Ar}-\mathrm{H}), 6.47$ (d, J=15.8 Hz, $1 \mathrm{H}$; olefin-H), $6.42(\mathrm{~d}, J=3.0 \mathrm{~Hz}, 1 \mathrm{H}, \mathrm{Ar}-\mathrm{H}), 6.15(\mathrm{dt}, J=15.8,6.7 \mathrm{~Hz}, 1 \mathrm{H}$, olefin-H), 3.75 $\left(\mathrm{s}, 3 \mathrm{H} ; \mathrm{N}-\mathrm{CH}_{3}\right), 2.20(\mathrm{q}, \mathrm{J}=7.0 \mathrm{~Hz}, 2 \mathrm{H}), 1.50-1.43(\mathrm{~m}, 2 \mathrm{H}), 1.34-1.27(\mathrm{~m}, 12 \mathrm{H}), 0.87(\mathrm{t}$, $J=6.7 \mathrm{~Hz}, 3 \mathrm{H}) ;{ }^{13} \mathrm{C} \mathrm{NMR}\left(100 \mathrm{MHz}, \mathrm{CDCl}_{3}\right) \delta 136.1(\mathrm{C}), 130.6(\mathrm{CH}), 129.6(\mathrm{C}), 129.1(\mathrm{CH})$, $128.7(\mathrm{C}), 128.3(\mathrm{CH}), 119.8(\mathrm{CH}), 118.5(\mathrm{CH}), 109.1(\mathrm{CH}), 101.0\left(\mathrm{CH}_{2}\right), 33.1\left(\mathrm{CH}_{2}\right), 32.9$ $\left(\mathrm{CH}_{3}\right), 31.9\left(\mathrm{CH}_{2}\right), 29.7\left(\mathrm{CH}_{2}\right), 29.6\left(\mathrm{CH}_{2} \times 2\right), 29.3\left(\mathrm{CH}_{2} \times 2\right), 22.7\left(\mathrm{CH}_{2}\right), 14.1\left(\mathrm{CH}_{3}\right) ; \mathrm{HRMS}$ (APCI): $m / z$ calcd for $\mathrm{C}_{20} \mathrm{H}_{30} \mathrm{~N}\left([\mathrm{M}+\mathrm{H}]^{+}\right)$: 284.2373, found 284.2371 . 


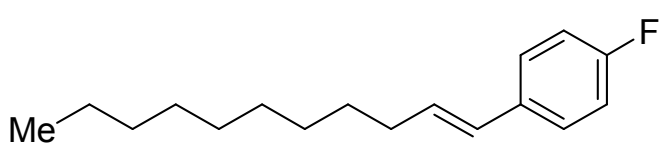

$(E)-3 n$

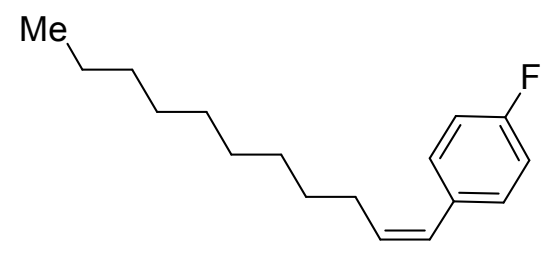

$(Z)-3 n$

(E)- and (Z)-1-Fluoro-4-(undec-1-enyl)benzene (3n). Compounds (E)- and (Z)-3n were synthesized from 1-methoxyundec-1-ene $1 \mathrm{a}(E / Z=55: 45,0.272 \mathrm{mmol}, 50 \mathrm{mg})$ and 2(4-fluorophenyl)-5,5-dimethyl-1,3,2-dioxaborinane 2 n (0.326 mmol, $68 \mathrm{mg}$ ) according to general procedure.Standard work-up afforded crude $3 \mathrm{n}$ as a mixture of $(E)$ - and $(Z)$ $3 n$ in a ratio $E / Z=95: 5$, and then purification by column chromatography on silica gel (eluent, petroleum ether/toluene $=20: 1$ ) yielded the title compound $3 \mathrm{n}$ as a clear oil (29 $\mathrm{mg}, 0.117 \mathrm{mmol}, 43 \%$ yield). Spectral data are only given for the major stereoisomer $(E)-\mathbf{3 n}$, which was isolated as a pure compound. $(E)-\mathbf{3 n}:{ }^{1} \mathrm{H}$ NMR (400 $\left.\mathrm{MHz}^{\mathrm{CDCl}} \mathrm{CD}_{3}\right) \delta 7.30-7.25(\mathrm{~m}, 2 \mathrm{H} ; \mathrm{Ar}-\mathrm{H}), 6.99-6.93(\mathrm{~m}, 2 \mathrm{H} ; \mathrm{Ar}-\mathrm{H}), 6.32(\mathrm{~d}, J=15.8 \mathrm{~Hz}$, $1 \mathrm{H}$; olefin-H), $6.12(\mathrm{dt}, J=15.8,6.9 \mathrm{~Hz}, 1 \mathrm{H}$; olefin-H), 2.17 (qd, J= 7.2, $1.2 \mathrm{~Hz}, 2 \mathrm{H}), 1.47-$ $1.40(\mathrm{~m}, 2 \mathrm{H}), 1.33-1.26(\mathrm{~m}, 12 \mathrm{H}), 0.87(\mathrm{t}, J=6.9 \mathrm{~Hz}, 3 \mathrm{H}) ;{ }^{13} \mathrm{C} \mathrm{NMR}\left(100 \mathrm{MHz}, \mathrm{CDCl}_{3}\right) \delta$ $161.8\left(C, d,{ }^{1} J_{C-F}=245.4 \mathrm{~Hz}, C-F\right), 134.1\left(C, d,{ }^{4} J_{C-F}=3.0 \mathrm{~Hz}, C-1^{\prime}\right), 131.0\left(C H, d,{ }^{5} C_{C-F}=\right.$ 2.2 Hz, olefin-CH), $128.5(\mathrm{CH}$, olefin- $\mathrm{CH}), 127.3\left(\mathrm{CH} \times 2, \mathrm{~d}^{3}{ }^{3} \mathrm{C}_{\mathrm{C}-\mathrm{F}}=7.7 \mathrm{~Hz}, \mathrm{C}-2^{\prime}\right.$ and $\left.\mathrm{C}-6^{\prime}\right)$, $115.3\left(\mathrm{CH} \times 2, \mathrm{~d},{ }^{2} J_{\mathrm{C}-\mathrm{F}}=22.0 \mathrm{~Hz}, \mathrm{C}-3^{\prime}\right.$ and $\left.\mathrm{C}-5^{\prime}\right), 33.0\left(\mathrm{CH}_{2}\right), 31.9\left(\mathrm{CH}_{2}\right), 29.6\left(\mathrm{CH}_{2}\right), 29.5$ $\left(\mathrm{CH}_{2}\right), 29.4\left(\mathrm{CH}_{2}\right), 29.3\left(\mathrm{CH}_{2}\right), 29.2\left(\mathrm{CH}_{2}\right), 22.7\left(\mathrm{CH}_{2}\right), 14.1\left(\mathrm{CH}_{3}\right) ; \mathrm{HRMS}(\mathrm{APCl}): \mathrm{m} / \mathrm{z}$ calcd for $\mathrm{C}_{17} \mathrm{H}_{24} \mathrm{~F}\left(\left[\mathrm{M}-\mathrm{H}_{2}+\mathrm{H}\right]^{+}\right):$:247.1857, found 247.1878.

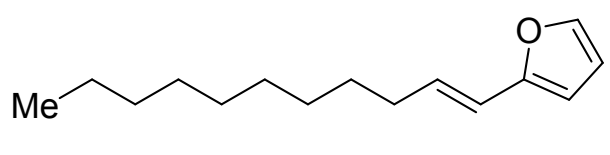

$(E)-30$

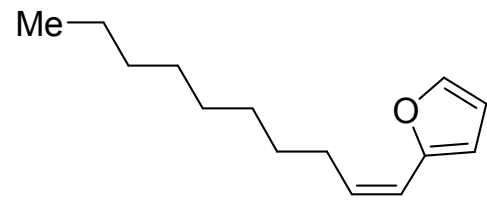

(Z)-30

(E)- and (Z)-2-(Undec-1-enyl)furan (30). Compounds (E)- and (Z)-30 were synthesized 
from 1-methoxyundec-1-ene $1 \mathrm{a}(E / Z=55: 45,0.272 \mathrm{mmol}, 50 \mathrm{mg})$ and 2-(furan-2-yl)5,5-dimethyl-1,3,2-dioxaborinane $20(0.326 \mathrm{mmol}, 59 \mathrm{mg})$ according to general procedure.Standard work-up afforded crude $\mathbf{3 0}$ as a mixture of $(E)$ - and (Z)-30 in a ratio $E / Z=82: 18$, and then purification by column chromatography on silica gel (eluent, petroleum ether/toluene $=20: 1$ ) yielded the title compound 30 as a pale-yellow oil. (25 mg, $0.114 \mathrm{mmol}, 41 \%$ yield). As (E)- and (Z)-3o are inseparable, NMR and MS data are given for the mixture of the two isomers. $(E)-30:{ }^{1} \mathrm{H} \mathrm{NMR}\left(400 \mathrm{MHz}, \mathrm{CDCl}_{3}\right) \delta 7.28$ (d, $J=2.0 \mathrm{~Hz}, 1 \mathrm{H} ; \mathrm{Ar}-\mathrm{H}), 6.32(\mathrm{dd}, J=3.0,2.0 \mathrm{~Hz}, 1 \mathrm{H} ; \mathrm{Ar}-\mathrm{H}), 6.23-6.20(\mathrm{~m}, 2 \mathrm{H}$; olefin$H), 6.10(\mathrm{~d}, J=3.0 \mathrm{~Hz}, 1 \mathrm{H} ; \operatorname{Ar}-\mathrm{H}), 2.14(\mathrm{dd}, J=12.8,7.1 \mathrm{~Hz}, 2 \mathrm{H}), 1.44-1.39(\mathrm{~m}, 2 \mathrm{H})$, $1.30-1.25(\mathrm{~m}, 12 \mathrm{H}), 0.86(\mathrm{t}, J=6.8 \mathrm{~Hz}, 3 \mathrm{H}) ;{ }^{13} \mathrm{C} \mathrm{NMR}\left(100 \mathrm{MHz}, \mathrm{CDCl}_{3}\right) \delta 153.4(\mathrm{C})$, 141.2 (CH), $130.4(\mathrm{CH}), 118.5(\mathrm{CH}), 111.1(\mathrm{CH}), 105.8(\mathrm{CH}), 32.8\left(\mathrm{CH}_{2}\right), 31.9\left(\mathrm{CH}_{2}\right), 29.6$ $\left(\mathrm{CH}_{2} \times 2\right), 29.4\left(\mathrm{CH}_{2}\right), 29.3\left(\mathrm{CH}_{2}\right), 29.2\left(\mathrm{CH}_{2}\right), 22.7\left(\mathrm{CH}_{2}\right), 14.2\left(\mathrm{CH}_{3}\right) ; \operatorname{HRMS}(\mathrm{APCl}): \mathrm{m} / \mathrm{z}$ calcd for $\mathrm{C}_{15} \mathrm{H}_{23} \mathrm{O}\left(\left[\mathrm{M}-\mathrm{H}_{2}+\mathrm{H}\right]^{+}\right)$: 219.1743, found 219.1730. (Z)-30: ${ }^{1} \mathrm{H}$ NMR (400 MHz, $\left.\mathrm{CDCl}_{3}\right) \delta 7.35(\mathrm{~d}, \mathrm{~J}=2.0 \mathrm{~Hz}, 1 \mathrm{H}), 6.37-6.36(\mathrm{~m}, 1 \mathrm{H}), 6.23(\mathrm{~d}, J=3.0 \mathrm{~Hz}, 1 \mathrm{H}), 6.20-6.18$ $(\mathrm{m}, 1 \mathrm{H}), 5.57--5.50(\mathrm{~m}, 1 \mathrm{H}), 2.43-2.38(\mathrm{~m}, 2 \mathrm{H}), 1.42-1.38(\mathrm{~m}, 2 \mathrm{H}), 1.29-1.26(\mathrm{~m}, 12 \mathrm{H})$, $0.85(\mathrm{t}, J=6.8 \mathrm{~Hz}, 3 \mathrm{H}) ;{ }^{13} \mathrm{C} \mathrm{NMR}\left(100 \mathrm{MHz}, \mathrm{CDCl}_{3}\right) \delta 152.3(\mathrm{C}), 141.2(\mathrm{CH}), 130.4(\mathrm{CH})$, 117.3 (CH), $111.1(\mathrm{CH}), 108.0(\mathrm{CH}), 32.8\left(\mathrm{CH}_{2}\right), 31.7\left(\mathrm{CH}_{2}\right), 29.6\left(\mathrm{CH}_{2} \times 2\right), 29.4\left(\mathrm{CH}_{2}\right)$, $29.3\left(\mathrm{CH}_{2}\right), 29.1\left(\mathrm{CH}_{2}\right), 22.6\left(\mathrm{CH}_{2}\right), 14.1\left(\mathrm{CH}_{3}\right) ; \mathrm{HRMS}(\mathrm{APCl}): \mathrm{m} / z$ calcd for $\mathrm{C}_{15} \mathrm{H}_{23} \mathrm{O}([\mathrm{M}$ $\left.\left.-\mathrm{H}_{2}+\mathrm{H}\right]^{+}\right):$219.1743, found 219.1730 .

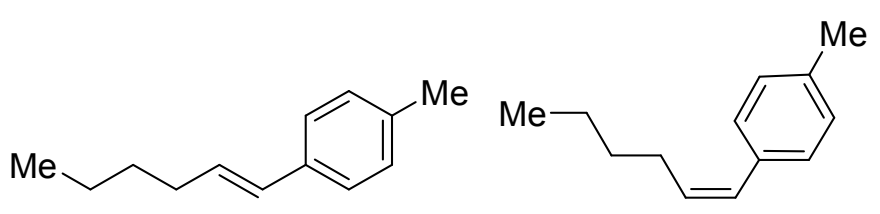

$(E)-3 p$

$(Z)-3 p$

$(E)$ - and (Z)-1-(Hex-1-enyl)-4-methylbenzene (3p). ${ }^{17}$ Compounds $(E)$ - and (Z)-3p were synthesized from 1-methoxyhex-1-ene $1 \mathbf{b}(E / Z=51: 49,0.439 \mathrm{mmol}, 50 \mathrm{mg})$ and 5,5- 
dimethyl-2-p-tolyl-1,3,2-dioxaborinane $2 \mathrm{a}(0.526 \mathrm{mmol}, 107 \mathrm{mg})$ according to general procedure. Standard work-up afforded crude $3 p$ as a mixture of $(E)$ - and (Z)-3p in a ratio $E / Z=95: 5$, and then purification by column chromatography on silica gel (eluent, petroleum ether/toluene $=20: 1$ ) yielded the title compound $\mathbf{3 p}$ as a clear oil $(63 \mathrm{mg}$, $0.362 \mathrm{mmol}, 82 \%$ yield). The (E)- and (Z)-isomers were partially separated by column chromatography. (E)-3p: ${ }^{1} \mathrm{H}$ NMR $\left(400 \mathrm{MHz}, \mathrm{CDCl}_{3}\right) \delta 7.21(\mathrm{~d}, J=8.1 \mathrm{~Hz}, 2 \mathrm{H} ; \mathrm{Ar}-\mathrm{H}), 7.07$ $(\mathrm{d}, J=8.0 \mathrm{~Hz}, 2 \mathrm{H} ; \mathrm{Ar}-\mathrm{H}), 6.32(\mathrm{~d}, J=15.8 \mathrm{~Hz}, 1 \mathrm{H}$; olefin-H), $6.15(\mathrm{dt}, J=15.8,6.9 \mathrm{~Hz}, 1 \mathrm{H}$; olefin-H), $2.30(\mathrm{~s}, 3 \mathrm{H}), 2.17(\mathrm{qd}, J=7.2,1.0 \mathrm{~Hz}, 2 \mathrm{H}), 1.46-1.31(\mathrm{~m}, 4 \mathrm{H}), 0.92(\mathrm{t}, J=7.2$ $\mathrm{Hz}, 3 \mathrm{H}) ;{ }^{13} \mathrm{C} \mathrm{NMR}\left(100 \mathrm{MHz}, \mathrm{CDCl}_{3}\right) \delta 136.4(\mathrm{C}), 135.2(\mathrm{C}), 130.2(\mathrm{CH}), 129.5(\mathrm{CH}), 129.1$ $(\mathrm{CH} \times 2), 125.8(\mathrm{CH} \times 2), 32.7\left(\mathrm{CH}_{2}\right), 31.6\left(\mathrm{CH}_{2}\right), 22.3\left(\mathrm{CH}_{2}\right), 22.1\left(\mathrm{CH}_{3}\right), 14.0\left(\mathrm{CH}_{3}\right) ; \mathrm{HRMS}$ (APCI): $m / z$ calcd for $\mathrm{C}_{13} \mathrm{H}_{19}\left([\mathrm{M}+\mathrm{H}]^{+}\right): 175.1481$, found 175.1489. (Z)-3p: ${ }^{1} \mathrm{H}$ NMR (400 $\left.\mathrm{MHz}^{\mathrm{CDCl}} \mathrm{CD}_{3}\right) \delta .17(\mathrm{~d}, J=8.1 \mathrm{~Hz}, 2 \mathrm{H} ; \mathrm{Ar}-\mathrm{H}), 7.12(\mathrm{~d}, J=8.0 \mathrm{~Hz}, 2 \mathrm{H} ; \mathrm{Ar}-\mathrm{H}), 6.36(\mathrm{~d}, J=$ $11.6 \mathrm{~Hz}, 1 \mathrm{H}$; olefin-H), 5.61 (dt, $J=11.6,7.2 \mathrm{~Hz}, 1 \mathrm{H}$; olefin-H), $2.33(\mathrm{~s}, 3 \mathrm{H}), 2.32(\mathrm{q}, J=$ 7.2, $1.0 \mathrm{~Hz}, 2 \mathrm{H}), 1.46-1.29(\mathrm{~m}, 4 \mathrm{H}), 0.87(\mathrm{t}, J=7.2 \mathrm{~Hz}, 3 \mathrm{H}) ;{ }^{13} \mathrm{C} \mathrm{NMR}\left(100 \mathrm{MHz}, \mathrm{CDCl}_{3}\right)$ $\delta 136.0(\mathrm{C}), 134.9(\mathrm{C}), 132.5(\mathrm{CH}), 128.8(\mathrm{CH} \times 2), 128.7(\mathrm{CH} \times 2), 128.5(\mathrm{CH}), 32.2\left(\mathrm{CH}_{2}\right)$, $28.4\left(\mathrm{CH}_{2}\right), 22.4\left(\mathrm{CH}_{2}\right), 22.1\left(\mathrm{CH}_{3}\right), 14.0\left(\mathrm{CH}_{3}\right) ; \mathrm{HRMS}(\mathrm{APCl}): \mathrm{m} / \mathrm{z}$ calcd for $\mathrm{C}_{13} \mathrm{H}_{19}([\mathrm{M}+$ $\left.H]^{+}\right)$: 175.1481, found 175.1489. Spectral data of $(E)$ - and $(Z)-3 p$ matched reported literature values. ${ }^{17}$

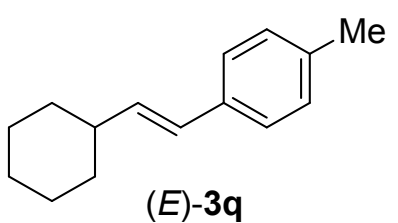

$(E)-3 q$

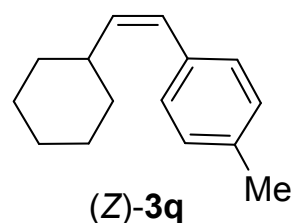

$(Z)-3 q$

$(E)$ - and (Z)-1-(2-Cyclohexylvinyl)-4-methylbenzene (3q). ${ }^{18}$ Compounds (E)- and (Z)3q were synthesized from (2-methoxyvinyl)cyclohexane $1 c(E / Z=67: 33,0.272 \mathrm{mmol}$, $38 \mathrm{mg}$ ) and 5,5-dimethyl-2-p-tolyl-1,3,2-dioxaborinane 2a $(0.326 \mathrm{mmol}, 67 \mathrm{mg})$ according to general procedure at $105^{\circ} \mathrm{C}$. Standard work-up crude $\mathbf{3 q}$ as a mixture of 
$(E)$ - and (Z)-3q in a ratio $E / Z=95: 5$, and then purification by column chromatography on silica gel (eluent, petroleum ether/toluene $=10: 1$ ) yielded the title compound $\mathbf{3 q}$ as a pale-yellow oil (39 mg, $0.195 \mathrm{mmol}, 72 \%$ yield). As (E)- and (Z)-3q are inseparable, NMR and MS data are given for the mixture of the two isomers. $(E)-3 q:{ }^{1} \mathrm{H}$ NMR (400 $\left.\left.\mathrm{MHz}^{\mathrm{CDCl}}\right)_{3}\right) \delta 7.25(\mathrm{~d}, J=8.0 \mathrm{~Hz}, 1 \mathrm{H} ; \mathrm{Ar}-\mathrm{H}), 7.10(\mathrm{~d}, J=8.0 \mathrm{~Hz}, 1 \mathrm{H} ; \mathrm{Ar}-\mathrm{H}), 6.32(\mathrm{~d}, J=$ $16.0 \mathrm{~Hz}, 1 \mathrm{H}$; olefin-H), 6.13 (dd, $J=16.0,6.9 \mathrm{~Hz}, 1 \mathrm{H}$; olefin-H), $2.33\left(\mathrm{~s}, 3 \mathrm{H} ; \mathrm{CH}_{3}\right), 2.16-$ $2.08(\mathrm{~m}, 1 \mathrm{H}), 1.82-1.76(\mathrm{~m}, 4 \mathrm{H}), 1.70-1.67(\mathrm{~m}, 1 \mathrm{H}), 1.38-1.14(\mathrm{~m}, 5 \mathrm{H}) ;{ }^{13} \mathrm{C}$ NMR $(100$ $\left.\mathrm{MHz}, \mathrm{CDCl}_{3}\right) \delta 136.4(\mathrm{C}), 135.8(\mathrm{CH}), 135.2(\mathrm{C}), 129.1(\mathrm{CH} \times 2), 127.0(\mathrm{CH}), 125.8(\mathrm{CH} \times$ 2), $41.1(\mathrm{CH}), 33.0\left(\mathrm{CH}_{2} \times 2\right), 26.2\left(\mathrm{CH}_{2}\right), 26.1\left(\mathrm{CH}_{2} \times 2\right), 21.1\left(\mathrm{CH}_{3}\right) ; \mathrm{HRMS}(\mathrm{APCl}): \mathrm{m} / \mathrm{z}$ calcd for $\mathrm{C}_{15} \mathrm{H}_{20}\left([\mathrm{M}+\mathrm{H}]^{+}\right): 201.1638$, found 201.1637. (Z)-3q: ${ }^{1} \mathrm{H}$ NMR $\left(400 \mathrm{MHz}, \mathrm{CDCl}_{3}\right)$ $\delta$ 7.18-7.16 (m, 5H; Ar-H), $6.28(\mathrm{~d}, J=11.9 \mathrm{~Hz}, 1 \mathrm{H}$; olefin-H), 5.45 (dd, $J=11.9,8.1 \mathrm{~Hz}$, $1 \mathrm{H}$; olefin-H), 2.60-2.56 (m, $1 \mathrm{H}), 2.35\left(\mathrm{~s}, 3 \mathrm{H} ; \mathrm{CH}_{3}\right), 1.74-1.71(\mathrm{~m}, 4 \mathrm{H}), 1.29-1.13(\mathrm{~m}$, $6 \mathrm{H}) ;{ }^{13} \mathrm{C} \mathrm{NMR}\left(100 \mathrm{MHz}, \mathrm{CDCl}_{3}\right) \delta 138.3(\mathrm{C}), 135.8(\mathrm{CH}), 135.2(\mathrm{C}), 128.9(\mathrm{CH} \times 2), 128.5$ $(\mathrm{CH}), 126.6(\mathrm{CH} \times 2), 39.5(\mathrm{CH}), 33.3\left(\mathrm{CH}_{2} \times 2\right), 26.3\left(\mathrm{CH}_{2} \times 2\right), 25.7\left(\mathrm{CH}_{2}\right), 21.1\left(\mathrm{CH}_{3}\right)$; HRMS (APCI): $m / z$ calcd for $\mathrm{C}_{15} \mathrm{H}_{20}\left([\mathrm{M}+\mathrm{H}]^{+}\right): 201.1638$, found 201.1637. Spectral data of $(E)$ - and (Z)-3q matched reported literature values. ${ }^{18}$
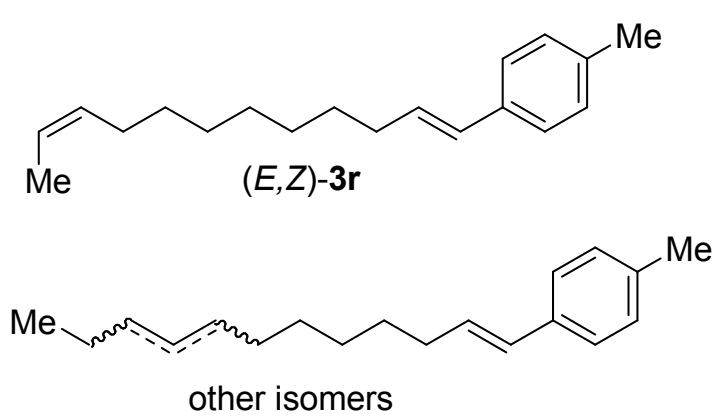

$(E, Z)-3 r /(E, E)-3 r /$ other isomers $=47: 37: 16$ olefin isomerization products (inseparable)<smiles>CCCCCCCCCC/C=C/c1ccc(C)cc1</smiles>

$(E)-3 r^{\prime}$

reduction product (separable)

Styrenes $(E, Z)-3 r,(E, E)-3 r$, other isomers and reduction product $(E)-3 r^{\prime}$. Compounds $(E, Z)-3 r,(E, E)-3 r$, other isomers and reduction product $(E)-3 r^{\prime}$ were synthesized from 
1-methoxydodeca-1,11-diene $1 \mathbf{d}(E / Z=54: 46,0.272 \mathrm{mmol}, 53 \mathrm{mg})$ and 5,5-dimethyl2-p-tolyl-1,3,2-dioxaborinane $2 \mathrm{a}(0.326 \mathrm{mmol}, 67 \mathrm{mg})$ according to general procedure. Standard work-up afforded crude $\mathbf{3 r}$ as a mixture of $(E, Z)-3 \mathbf{r},(E, E)-\mathbf{3 r}$, and other isomers in a ratio 47:37:16, and along with partial reduction product $(E)-\mathbf{3 r}$ '. Purification by column chromatography on silica gel (eluent, petroleum ether/toluene $=20: 1)$ yielded a mixture of $(E, Z)-3 r,(E, E)-3 r$ and other isomers as a clear oil $(55 \mathrm{mg}$, $0.215 \mathrm{mmol}, 79 \%$ yield), as well as the reduction product $(E)-3 r^{\prime}$ as a clear oil $(9 \mathrm{mg}$, $0.034 \mathrm{mmol}, 12 \%$ yield, $E / Z>95: 5)$. As the six sets of signals for $(E, Z)-3 \mathrm{r},(E, E)-3 \mathrm{r}$ and other isomers in ${ }^{1} \mathrm{H}$ and ${ }^{13} \mathrm{C}$ NMR are not well differentiated and could not be unequivocally attributed, NMR and MS data are given for the mixture of the six isomers. For reduction product $(E)-3 r^{\prime}$ which was isolated as a pure compound as a pale-yellow oil, and spectra data matched reported literature values. ${ }^{19}$ For a mixture of $(E, Z)-3 r,(E, E)-3 r$ and other isomers: ${ }^{1} \mathrm{H}$ NMR $\left(400 \mathrm{MHz}, \mathrm{CDCl}_{3}\right) \delta 7.24(\mathrm{~d}, J=8.0 \mathrm{~Hz}$, $2 \mathrm{H} ; \operatorname{Ar}-\mathrm{H}), 7.09(\mathrm{~d}, J=8.0 \mathrm{~Hz}, 2 \mathrm{H} ; \mathrm{Ar}-\mathrm{H}), 6.34(\mathrm{~d}, J=15.8 \mathrm{~Hz}, 1 \mathrm{H}$; olefin-H), $6.16(\mathrm{dt}, J=$ 15.8, 6.9 Hz, 1H; olefin-H), 5.45-5.34 (m, 2H; olefin-H), $2.32(\mathrm{~s}, 3 \mathrm{H}), 2.18(\mathrm{q}, J=7.2 \mathrm{~Hz}$, 2H), 2.04-1.94 (m, 2.4H), $1.64\left(\mathrm{dd}, J=3.5,1.1 \mathrm{~Hz}, 1.4 \mathrm{H}\right.$; vinyl- $\mathrm{CH}_{3}$ for $\left.(E, Z)-3 \mathrm{r}\right), 1.61$ (d, $J=5.7 \mathrm{~Hz}, 1.1 \mathrm{H}$; vinyl- $\mathrm{CH}_{3}$ for $\left.(E, E)-3 \mathrm{r}\right), 1.47-1.42(\mathrm{~m}, 2 \mathrm{H}), 1.35-1.26(\mathrm{~m}, 8 \mathrm{H}), 0.97-$ $0.94\left(\mathrm{~m}, 0.5 \mathrm{H}\right.$; terminal- $\mathrm{CH}_{3}$ for other isomers); As other isomers in ${ }^{13} \mathrm{C}$ NMR could not be unequivocally attributed, ${ }^{13} C$ NMR assignment is only given for the mixture of $(E, Z)$ and $(E, E)-3 r .{ }^{13} \mathrm{C} \mathrm{NMR}\left(100 \mathrm{MHz}, \mathrm{CDCl}_{3}\right) \delta 136.4(\mathrm{C}), 135.2(\mathrm{C}), 131.6(\mathrm{CH}), 130.9(\mathrm{CH})$, $130.2(\mathrm{CH}), 129.5(\mathrm{CH}), 129.1(\mathrm{CH} \times 2), 125.8(\mathrm{CH} \times 2), 124.5(\mathrm{CH}), 123.6(\mathrm{CH}), 33.0$ $\left(\mathrm{CH}_{2}\right), 32.6\left(\mathrm{CH}_{2}\right), 32.5\left(\mathrm{CH}_{2}\right), 29.7\left(\mathrm{CH}_{2}\right), 29.6\left(\mathrm{CH}_{2}\right), 29.5\left(\mathrm{CH}_{2}\right), 29.4\left(\mathrm{CH}_{2} \times 2\right), 29.2\left(\mathrm{CH}_{2}\right.$ $\times 3), 29.1\left(\mathrm{CH}_{2}\right), 29.0\left(\mathrm{CH}_{2}\right), 26.8\left(\mathrm{CH}_{2}\right), 22.1\left(\mathrm{Ph}-\mathrm{CH}_{3}\right), 14.0$ (vinyl- $\mathrm{CH}_{3}$ for $\left.(E, \mathrm{Z})-3 \mathrm{r}\right), 14.0$ (vinyl- $\mathrm{CH}_{3}$ for $(E, E)-3 r$ ); HRMS (APCl): $m / z$ calcd for $\mathrm{C}_{18} \mathrm{H}_{29}\left(\left[[\mathrm{M}+\mathrm{H}]^{+}\right): 257.2264\right.$, found 257.2257. Reduction product (E)-3r': ${ }^{1} \mathrm{H}$ NMR $\left(400 \mathrm{MHz}, \mathrm{CDCl}_{3}\right) \delta 7.22(\mathrm{~d}, J=8.1 \mathrm{~Hz}$, $2 \mathrm{H} ; \mathrm{Ar}-\mathrm{H}), 7.07$ (d, J = 7.2 Hz, 2H; Ar-H), 6.32 (d, J = $15.8 \mathrm{~Hz}, 1 \mathrm{H}$; olefin-H), 6.15 (dt, $J=$ 
$15.8,6.9 \mathrm{~Hz}, 1 \mathrm{H}$; olefin-H), $2.30(\mathrm{~s}, 3 \mathrm{H}), 2.16(\mathrm{qd}, J=7.2,1.0 \mathrm{~Hz}, 2 \mathrm{H}), 1.47-1.42(\mathrm{~m}, 2 \mathrm{H})$, 1.28-1.25 (m, 14H), $0.86(\mathrm{t}, J=7.2 \mathrm{~Hz}, 3 \mathrm{H}) ;{ }^{13} \mathrm{C} \mathrm{NMR}\left(100 \mathrm{MHz}, \mathrm{CDCl}_{3}\right) \delta 136.4(\mathrm{C})$, $135.2(\mathrm{C}), 130.2(\mathrm{CH}), 129.5(\mathrm{CH}), 129.1(\mathrm{CH} \times 2), 125.8(\mathrm{CH} \times 2), 33.0\left(\mathrm{CH}_{2}\right), 31.9\left(\mathrm{CH}_{2}\right)$, $29.6\left(\mathrm{CH}_{2} \times 2\right), 29.5\left(\mathrm{CH}_{2}\right), 29.4\left(\mathrm{CH}_{2}\right), 29.3\left(\mathrm{CH}_{2}\right), 29.2\left(\mathrm{CH}_{2}\right), 22.7\left(\mathrm{CH}_{2}\right), 22.1\left(\mathrm{CH}_{3}\right), 14.1$ $\left(\mathrm{CH}_{3}\right)$.

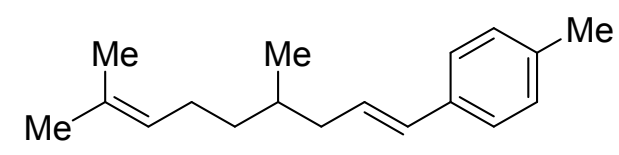

$(E)-3 s$<smiles>CC(C)=CCCC(C)C/C=C\c1ccc(C)cc1</smiles>

(E)- and (Z)-1-Methyl-4-(4,8-dimethylnona-1,7-dienyl)benzene (3s). Compounds (E)and (Z)-3s were synthesized from 1-methoxy-4,8-dimethylnona-1,7-diene 1e $(E / Z=$ $55: 45,0.275 \mathrm{mmol}, 50 \mathrm{mg}$ ) and 5,5-dimethyl-2-p-tolyl-1,3,2-dioxaborinane 2a (0.329 $\mathrm{mmol}, 67 \mathrm{mg}$ ) according to general procedure at $105^{\circ} \mathrm{C}$. Standard work-up afforded crude $3 \mathrm{~s}$ as a mixture of $(E)$ - and $(Z)-3 \mathrm{~s}$ in a ratio $E / Z=96: 4$, and then purification by column chromatography on silica gel (eluent, petroleum ether/toluene $=20: 1$ ) yielded the title compound $3 \mathrm{~s}$ as a clear oil (51 $\mathrm{mg}, 0.212 \mathrm{mmol}, 77 \%$ yield). Spectral data are only given for the major stereoisomer $(E)$-3s, which was isolated as a pure compound. (E)-3s: ${ }^{1} \mathrm{H}$ NMR $\left(400 \mathrm{MHz}, \mathrm{CDCl}_{3}\right) \delta 7.23(\mathrm{~d}, J=7.9 \mathrm{~Hz}, 2 \mathrm{H} ; \mathrm{Ar}-\mathrm{H}), 7.09$ (d, J = 7.9 Hz, 2H; Ar-H), $6.32(\mathrm{~d}, J=15.8 \mathrm{~Hz}, 1 \mathrm{H}$; olefin-H), $6.20-6.12(\mathrm{~m}, 1 \mathrm{H}$; olefin-H), $5.10(\mathrm{t}, J=7.1 \mathrm{~Hz}$, $1 \mathrm{H}$; olefin-H), $2.35(\mathrm{~s}, 3 \mathrm{H}), 2.33-2.10(\mathrm{~m}, 1 \mathrm{H}), 2.08-1.97(\mathrm{~m}, 3 \mathrm{H}), 1.69(\mathrm{~s}, 3 \mathrm{H}), 1.62(\mathrm{~s}$, $3 \mathrm{H}), 1.59-1.55(\mathrm{~m}, 1 \mathrm{H}), 1.45-1.39(\mathrm{~m}, 1 \mathrm{H}), 1.22-1.13(\mathrm{~m}, 1 \mathrm{H}), 0.91(\mathrm{~d}, J=7.6 \mathrm{~Hz}, 3 \mathrm{H})$; ${ }^{13} \mathrm{C} \mathrm{NMR}\left(100 \mathrm{MHz}, \mathrm{CDCl}_{3}\right) \delta 136.4(\mathrm{C}), 135.1(\mathrm{C}), 131.1(\mathrm{C}), 130.7(\mathrm{CH}), 129.1(\mathrm{CH} \times 2)$, $128.6(\mathrm{CH}), 125.8(\mathrm{CH} \times 2), 124.8(\mathrm{CH}), 40.5\left(\mathrm{CH}_{2}\right), 36.7\left(\mathrm{CH}_{2}\right), 32.9(\mathrm{CH}), 25.7\left(\mathrm{CH}_{3}\right)$, $25.6\left(\mathrm{CH}_{3}\right), 22.1\left(\mathrm{CH}_{3}\right), 19.5\left(\mathrm{CH}_{2}\right), 17.6\left(\mathrm{CH}_{3}\right) ; \mathrm{HRMS}(\mathrm{APCl}): \mathrm{m} / \mathrm{z}$ calcd for $\mathrm{C}_{18} \mathrm{H}_{27}([\mathrm{M}+$ $\left.H]^{+}\right): 243.2107$, found 243.2115 . 
<smiles>Cc1ccc(/C=C/CCc2ccccc2)cc1</smiles>

$(E)-3 t$<smiles>Cc1ccc(/C=C\CCc2ccccc2)cc1</smiles>

$(Z)-3 t$

(E)- and (Z)-1-Methyl-4-(4-phenylbut-1-enyl)benzene (3t). ${ }^{20}$ Compounds $(E)$ - and (Z)3t were synthesized from 1-(4-methoxybut-3-enyl)benzene 1f $(E / Z=58: 42,0.272$ $\mathrm{mmol}, 44 \mathrm{mg}$ ) and 5,5-dimethyl-2-p-tolyl-1,3,2-dioxaborinane $2 \mathrm{a}$ (0.326 mmol, $66 \mathrm{mg}$ ) according to general procedure. Standard work-up afforded crude 3t as a mixture of $(E)$ - and $(Z)-3 t$ in a ratio $E / Z=96: 4$, and then purification by column chromatography on silica gel (eluent, petroleum ether/toluene $=15: 1$ ) yielded the title compound $\mathbf{3 t}$ as a pale-yellow oil (54 mg, $0.243 \mathrm{mmol}, 90 \%$ yield). Spectral data are only given for the major stereoisomer $(E)-\mathbf{3 t}$, which was isolated as a pure compound. $(E)-\mathbf{3 t}$ : ${ }^{1} \mathrm{H}$ NMR $\left(400 \mathrm{MHz}, \mathrm{CDCl}_{3}\right) \delta 7.38-7.26(\mathrm{~m}, 7 \mathrm{H} ; \mathrm{Ar}-\mathrm{H}), 7.16(\mathrm{~d}, J=8.0 \mathrm{~Hz}, 2 \mathrm{H} ; \mathrm{Ar}-\mathrm{H}), 6.45(\mathrm{~d}, J=$ $15.8 \mathrm{~Hz}, 1 \mathrm{H}$; olefin-H), 6.27 (dt, $J=15.8,6.8 \mathrm{~Hz}, 1 \mathrm{H}$; olefin-H), 2.87-2.83 (m, 2H), 2.58 $(\mathrm{dd}, J=15.0,7.1 \mathrm{~Hz}, 2 \mathrm{H}), 2.39\left(\mathrm{~s}, 3 \mathrm{H} ; \mathrm{CH}_{3}\right) ;{ }^{13} \mathrm{CNMR}\left(100 \mathrm{MHz}, \mathrm{CDCl}_{3}\right) \delta 141.8(\mathrm{C}), 136.6$ (C), $134.9(\mathrm{C}), 130.2(\mathrm{CH}), 129.2(\mathrm{CH} \times 2), 128.9(\mathrm{CH}), 128.5(\mathrm{CH} \times 2), 128.3(\mathrm{CH} \times 3)$, $125.9(\mathrm{CH}), 125.8(\mathrm{CH}), 35.9\left(\mathrm{CH}_{2}\right), 34.9\left(\mathrm{CH}_{2}\right), 21.1\left(\mathrm{CH}_{3}\right)$. Spectral data of $(E)-3 \mathrm{t}$ matched reported literature values..$^{20}$<smiles>Cc1ccc(/C=C/C(c2ccccc2)c2ccccc2)cc1</smiles>

$(E)-3 \mathbf{u}$<smiles>C/C(=C\Cc1ccc(C)cc1)c1ccccc1</smiles>

$(E)-3 \mathbf{u}^{\prime}$

(E)-1-Methyl-4-(3-phenylbut-1-enyl)benzene $\quad(E-3 u)^{21} \quad$ and $\quad(2 E)-1-m e t h y l-4-(3-$ phenyl-2-buten-1-yl)benzene ( $\left.E-3 u^{\prime}\right) .{ }^{22}$ Compounds $E-3 u$ and $E-3 u^{\prime}$ were synthesized from 1-(4-methoxybut-3-en-2-yl)benzene $1 \mathrm{~g}(E / Z=60: 40,0.272 \mathrm{mmol}, 44 \mathrm{mg})$ and 
5,5-dimethyl-2-p-tolyl-1,3,2-dioxaborinane $2 \mathrm{a}(0.326 \mathrm{mmol}, 66 \mathrm{mg})$ according to general procedure at $105^{\circ} \mathrm{C}$. Standard work-up afforded crude product as a mixture of $(E)-3 \mathbf{u}$ and $(E)-3 \mathbf{u}^{\prime}$ in a ratio $86: 14$, and then purification by column chromatography on silica gel (eluent, petroleum ether/toluene $=15: 1)$ yielded a mixture of $(E)-3 \mathbf{u}(E / Z$ $>95: 5)$ and an inseparable $(E)-3 u^{\prime}(E / Z>95: 5)$ as a clear oil (43 mg, $0.194 \mathrm{mmol}, 71 \%$ yield). As (E)-3u and (E)-3 ' ' are inseparable, NMR data are given for the mixture of the two isomers. (E)-3u: ${ }^{1} \mathrm{H}$ NMR $\left(400 \mathrm{MHz}, \mathrm{CDCl}_{3}\right) \delta 7.35-7.20(\mathrm{~m}, 7 \mathrm{H} ; \mathrm{Ar}-\mathrm{H}), 7.13(\mathrm{~d}, J=$ $7.9 \mathrm{~Hz}, 2 \mathrm{H} ; \mathrm{Ar}-\mathrm{H}), 6.42-6.31(\mathrm{~m}, 2 \mathrm{H}$; olefin-H), 3.67-3.53 (m, $1 \mathrm{H}), 2.33\left(\mathrm{~s}, 3 \mathrm{H} ; \mathrm{CH}_{3}\right)$, $1.47\left(\mathrm{~d}, J=7.1 \mathrm{~Hz}, 3 \mathrm{H} ; \mathrm{CH}_{3}\right) ;{ }^{13} \mathrm{C} \mathrm{NMR}\left(100 \mathrm{MHz}, \mathrm{CDCl}_{3}\right) \delta 145.8(\mathrm{C}), 136.7(\mathrm{C}), 134.8(\mathrm{C})$, 134.2 (CH), $129.2(\mathrm{CH} \times 2), 128.5(\mathrm{CH} \times 2), 128.4(\mathrm{CH}), 127.3(\mathrm{CH} \times 2), 126.2(\mathrm{CH}), 126.1$ $(\mathrm{CH} \times 2), 42.6(\mathrm{CH}), 21.3\left(\mathrm{CH}_{3}\right), 21.2\left(\mathrm{CH}_{3}\right)$. Spectral data of $(E)-3 \mathrm{u}$ matched reported literature values. ${ }^{21}(E)-3 u^{\prime}:{ }^{1} \mathrm{H}$ NMR $\left(400 \mathrm{MHz}, \mathrm{CDCl}_{3}\right) \delta 7.49(\mathrm{~d}, J=7.4 \mathrm{~Hz}, 2 \mathrm{H} ; \mathrm{Ar}-\mathrm{H})$, 7.35-7.20 (m, 5H; Ar-H), 7.14-7.09 (m, 2H; Ar-H), 6.05 (t, J = 7.3 Hz, 1H; olefin-H), 3.62 (d, J = 7.3 Hz, 1H), $2.36\left(\mathrm{~s}, 3 \mathrm{H} ; \mathrm{CH}_{3}\right), 2.15\left(\mathrm{~s}, 3 \mathrm{H} ; \mathrm{CH}_{3}\right) ;{ }^{13} \mathrm{C} \mathrm{NMR}\left(100 \mathrm{MHz}, \mathrm{CDCl}_{3}\right) \delta 143.7$ (C), $137.9(\mathrm{C}), 135.4(\mathrm{C} \times 2), 128.3(\mathrm{CH} \times 2), 128.1(\mathrm{CH} \times 2), 128.04(\mathrm{CH}), 127.0(\mathrm{CH})$, $126.7(\mathrm{CH} \times 2), 125.7(\mathrm{CH} \times 2), 34.5\left(\mathrm{CH}_{2}\right), 21.1\left(\mathrm{CH}_{3}\right), 15.9\left(\mathrm{CH}_{3}\right)$.

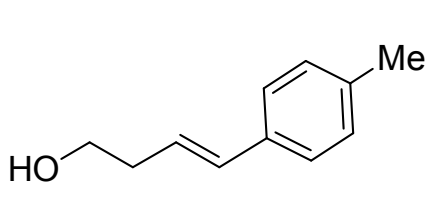

$(E)-3 v$

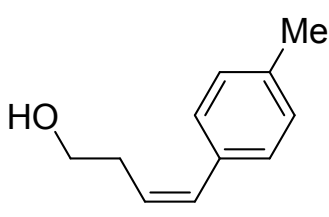

(Z)-3v

(E)- and (Z)-4-(4-Methylphenyl)-3-buten-1-ol (3v). ${ }^{23,24}$ Compounds (E)- and (Z)-3v were synthesized from 2,3-dihydrofuran $1 \mathrm{~h}(0.271 \mathrm{mmol}, 19 \mathrm{mg})$ and 5,5-dimethyl-2p-tolyl-1,3,2-dioxaborinane 2a $(0.326 \mathrm{mmol}, 66 \mathrm{mg})$ according to general procedure. Standard work-up afforded crude product as a mixture of $(E)$ - and (Z)-3v in a ratio $E / Z$ $=89: 11$, and then purification by column chromatography on silica gel (eluent, 
petroleum ether/ethyl acetate $=9: 1$ to $6: 1)$ yielded $(E)$ - and $(Z)-3 \mathbf{v}$ as a white solid $(27$ mg, 0.169 mmol, $62 \%$ yield). (E)-3v: ${ }^{1} \mathrm{H} \mathrm{NMR}\left(400 \mathrm{MHz}, \mathrm{CDCl}_{3}\right) \delta 7.24(\mathrm{~d}, J=8.0 \mathrm{~Hz}, 2 \mathrm{H}$; Ar-H), $7.10(\mathrm{~d}, J=8.0 \mathrm{~Hz}, 2 \mathrm{H} ; \mathrm{Ar}-\mathrm{H}), 6.45(\mathrm{~d}, J=15.9 \mathrm{~Hz}, 1 \mathrm{H}$; olefin-H), 6.13 (dt, J = 15.9, $7.2 \mathrm{~Hz}, 1 \mathrm{H}$; olefin-H), $3.73(\mathrm{t}, J=6.3 \mathrm{~Hz}, 2 \mathrm{H}), 2.46(\mathrm{q}, J=6.6 \mathrm{~Hz}, 2 \mathrm{H}), 2.31(\mathrm{~s}, 3 \mathrm{H}), 1.65$ (brs, $1 \mathrm{H}) ;{ }^{13} \mathrm{C} \mathrm{NMR}\left(100 \mathrm{MHz}, \mathrm{CDCl}_{3}\right) \delta 137.0$ (C), $134.4(\mathrm{C}), 132.7(\mathrm{CH}), 129.2(\mathrm{CH} \times 2)$, $125.9(\mathrm{CH} \times 2), 125.2(\mathrm{CH}), 62.0\left(\mathrm{CH}_{2}\right), 36.4\left(\mathrm{CH}_{2}\right), 21.1\left(\mathrm{CH}_{3}\right) ; \mathrm{HRMS}(\mathrm{APCl}): \mathrm{m} / z$ calcd for $\mathrm{C}_{11} \mathrm{H}_{15} \mathrm{O}\left([\mathrm{M}+\mathrm{H}]^{+}\right):$163.1117, found 163.1121. Spectral data of $(E)-3 \mathbf{v}$ matched reported literature values. ${ }^{23}(\mathrm{Z})-3 \mathrm{v}:{ }^{1} \mathrm{H}$ NMR $\left(400 \mathrm{MHz}, \mathrm{CDCl}_{3}\right) \delta 7.19(\mathrm{~d}, J=8.0 \mathrm{~Hz}, 2 \mathrm{H}$; Ar-H), $7.12(\mathrm{~d}, J=8.0 \mathrm{~Hz}, 2 \mathrm{H} ; \mathrm{Ar}-\mathrm{H}), 6.53(\mathrm{~d}, J=11.7 \mathrm{~Hz}, 1 \mathrm{H}$; olefin-H), 5.62 (dt, $J=11.6$, $7.4 \mathrm{~Hz}, 1 \mathrm{H}$; olefin-H), $3.73(\mathrm{t}, J=6.5 \mathrm{~Hz}, 2 \mathrm{H}), 2.60(\mathrm{qd}, J=7.0,1.5 \mathrm{~Hz}, 2 \mathrm{H}), 2.32(\mathrm{~s}, 3 \mathrm{H})$; ${ }^{13} \mathrm{C} \mathrm{NMR}\left(100 \mathrm{MHz}, \mathrm{CDCl}_{3}\right) \delta 136.6(\mathrm{C}), 134.3(\mathrm{C}), 131.5(\mathrm{CH}), 128.9(\mathrm{CH} \times 2), 128.6(\mathrm{CH}$ $\times 2), 127.4(\mathrm{CH}), 62.6\left(\mathrm{CH}_{2}\right), 32.0\left(\mathrm{CH}_{2}\right), 21.2\left(\mathrm{CH}_{3}\right)$. Spectral data of $(\mathrm{Z})-3 \mathrm{v}$ matched reported literature values. ${ }^{24}$<smiles>Cc1ccc(/C=C/c2ccccc2)cc1</smiles>

$(E)-3 \mathbf{w}$

(E)-1-Methyl-4-styrylbenzene $(3 w) .^{7}$ Compound $3 w$ was synthesized from 1-(2methoxyvinyl)benzene $\mathbf{1 i}(E / Z=55: 45,0.373 \mathrm{mmol}, 50 \mathrm{mg})$ and 5,5-dimethyl-2-p-tolyl1,3,2-dioxaborinane $2 \mathrm{a}(0.448 \mathrm{mmol}, 91 \mathrm{mg})$ according to general procedure. Standard work-up afforded crude product containing the $(E)-3 w$ as a single diastereomer (as determined by ${ }^{1} \mathrm{H} N \mathrm{NMR}$ ), and then purification by column chromatography on silica gel (eluent, petroleum ether/toluene $=10: 1$ ) yielded $(E)-3 \mathbf{w}$ as a white solid (59 mg, $0.304 \mathrm{mmol}, 81 \%$ yield). (E)-3w: ${ }^{1} \mathrm{H} \mathrm{NMR}\left(400 \mathrm{MHz}, \mathrm{CDCl}_{3}\right) \delta$ 7.50-7.48 (m, 2H), 7.41-7.39 (m, 2H), 7.36-7.30 (m, 2H), 7.23-7.21 (m, 1H), $7.15(\mathrm{~d}, J$ $=7.9 \mathrm{~Hz}, 2 \mathrm{H}), 7.09\left(\mathrm{~d}, J_{A B}=16.4 \mathrm{~Hz}, 1 \mathrm{H}\right), 7.04\left(\mathrm{~d}, J_{A B}=16.4 \mathrm{~Hz}, 1 \mathrm{H}\right), 2.35(\mathrm{~s}, 3 \mathrm{H}) ;{ }^{13} \mathrm{C}$ 
NMR $\left(100 \mathrm{MHz}, \mathrm{CDCl}_{3}\right) \delta 137.5(\mathrm{C} \times 2), 134.5(\mathrm{C}), 129.4(\mathrm{CH} \times 2), 128.6(\mathrm{CH} \times 3), 127.7$ $(\mathrm{CH}), 127.4(\mathrm{CH}), 126.6(\mathrm{CH} \times 4), 21.2\left(\mathrm{CH}_{3}\right)$. Spectral data of $(E)-3 \mathbf{w}$ matched reported literature values. ${ }^{7}$<smiles>Cc1ccc(/C=C/c2ccc(N(C)C)cc2)cc1</smiles>

$(E)-\mathbf{3 x}$

(E)-N,N-Dimethyl-4-(4-methylstyryl)aniline $(3 x) .{ }^{7}$ Compound $3 x$ was synthesized from 4-(2-methoxyvinyl)-N,N-dimethylbenzenamine $1 \mathrm{j}(E / Z=50: 50,0.274 \mathrm{mmol}, 48$ $\mathrm{mg}$ ) and 5,5-dimethyl-2-p-tolyl-1,3,2-dioxaborinane $\mathbf{2 a}(0.326 \mathrm{mmol}, 67 \mathrm{mg})$ according to general procedure. Standard work-up afforded crude product containing the $(E)$-3x as a single diastereomer (as determined by ${ }^{1} \mathrm{H} N \mathrm{NM}$ ), and then purification by column chromatography on silica gel (eluent, petroleum ether/ethyl acetate $=6: 1$ ) yielded $(E)$ $3 \mathbf{x}$ as a white solid (57 mg, $0.241 \mathrm{mmol}, 88 \%$ yield). (E)-3x: ${ }^{1} \mathrm{H} \mathrm{NMR}\left(400 \mathrm{MHz}, \mathrm{C}_{6} \mathrm{D}_{6}\right) \delta$ $7.44(\mathrm{~d}, J=8.7 \mathrm{~Hz}, 2 \mathrm{H}), 7.37(\mathrm{~d}, J=8.0 \mathrm{~Hz}, 2 \mathrm{H}), 7.18-7.15(\mathrm{~m}, 1 \mathrm{H}), 7.08-7.02(\mathrm{~m}, 3 \mathrm{H})$, $6.59(\mathrm{~d}, \mathrm{~J}=8.7 \mathrm{~Hz}, 2 \mathrm{H}), 2.51\left(\mathrm{~s}, 6 \mathrm{H} ; N\left(\mathrm{CH}_{3}\right)_{2}\right), 2.15\left(\mathrm{~s}, 3 \mathrm{H} ; \mathrm{CH}_{3}\right) ;{ }^{13} \mathrm{C} \mathrm{NMR}\left(100 \mathrm{MHz}, \mathrm{C}_{6} \mathrm{D}_{6}\right)$ $\delta 150.5(\mathrm{C}), 136.4(\mathrm{C}), 136.1(\mathrm{C}), 129.6(\mathrm{CH} \times 2), 128.6(\mathrm{CH}), 127.9(\mathrm{CH} \times 2), 126.7(\mathrm{C})$, $126.5(\mathrm{CH} \times 2), 124.9(\mathrm{CH}), 112.9(\mathrm{CH} \times 2), 40.0\left(\mathrm{CH}_{3} \times 2\right), 21.2\left(\mathrm{CH}_{3}\right)$. Spectral data of (E)-3x matched reported literature values. ${ }^{7}$<smiles>COC(=O)c1ccc(/C=C/c2ccc(C)cc2)cc1</smiles>

(E)-Methyl 4-(4-methylstyryl)benzoate (3y). ${ }^{12}$ Compound 3y was synthesized from methyl 4-(2-methoxyvinyl)benzoate $1 \mathbf{k}(E / Z=30: 70,0.274 \mathrm{mmol}, 53 \mathrm{mg})$ and 5,5- 
dimethyl-2-p-tolyl-1,3,2-dioxaborinane $2 \mathrm{a}(0.326 \mathrm{mmol}, 67 \mathrm{mg})$ according to general procedure at $105^{\circ} \mathrm{C}$. Standard work-up afforded crude product containing the (E)-3y as a single diastereomer (as determined by ${ }^{1} \mathrm{H} N \mathrm{NMR}$ ), and then purification by column chromatography on silica gel (eluent, petroleum ether/ethyl acetate $=6: 1$ ) yielded $(E)$ 3y as a white solid (31 mg, $0.125 \mathrm{mmol}, 45 \%$ yield). (E)-3y: ${ }^{1} \mathrm{H} \mathrm{NMR} \mathrm{(400} \mathrm{MHz,} \mathrm{CDCl}_{3}$ ) $\delta 8.07(\mathrm{~d}, J=8.1 \mathrm{~Hz}, 2 \mathrm{H}), 7.60(\mathrm{~d}, J=8.1 \mathrm{~Hz}, 2 \mathrm{H}), 7.49(\mathrm{~d}, J=8.0 \mathrm{~Hz}, 2 \mathrm{H}), 7.31-7.11(\mathrm{~m}$, 4H), 3.97 (s, 3H), $2.42(\mathrm{~s}, 3 \mathrm{H}) ;{ }^{13} \mathrm{C}$ NMR (100 MHz, $\left.\mathrm{CDCl}_{3}\right) \delta 166.9$ (C), 142.0 (C), 138.3 (C), $134.0(\mathrm{C}), 131.2(\mathrm{CH}), 130.0(\mathrm{CH} \times 2), 129.5(\mathrm{CH} \times 2), 128.7(\mathrm{C}), 126.7(\mathrm{CH} \times 2)$, $126.5(\mathrm{CH}), 126.2(\mathrm{CH} \times 2), 52.0\left(\mathrm{CH}_{3}\right), 21.3\left(\mathrm{CH}_{3}\right)$. Spectral data of $(E)$-3y matched reported literature values. ${ }^{12}$<smiles>COc1ccc2cc(/C=C/c3ccc(C)cc3)ccc2c1</smiles>

(E)-2-Methoxy-6-(4-methylstyryl)naphthalene (3z). ${ }^{12}$ Compound $3 z$ was synthesized from 2-methoxy-6-(2-methoxyvinyl)naphthalene 1 I ( $E / Z=65: 35,0.276 \mathrm{mmol}, 59 \mathrm{mg})$ and 5,5-dimethyl-2-p-tolyl-1,3,2-dioxaborinane $2 \mathrm{a}(0.331 \mathrm{mmol}, 67 \mathrm{mg})$ according to general procedure. Standard work-up afforded crude product containing the $(E)-\mathbf{3 z}$ as a single diastereomer (as determined by ${ }^{1} \mathrm{H} N \mathrm{NMR}$ ), and then purification by column chromatography on silica gel (eluent, petroleum ether/ $\mathrm{CH}_{2} \mathrm{Cl}_{2}=6: 1$ to $2: 1$ ) yielded $(E)$ $3 z$ as a white solid (59 mg, $0.215 \mathrm{mmol}, 78 \%$ yield). (E)-3z: ${ }^{1} \mathrm{H} \mathrm{NMR}\left(400 \mathrm{MHz}, \mathrm{CDCl}_{3}\right) \delta$ 7.76-7.69 (m, 4H), $7.43(\mathrm{~d}, J=8.0 \mathrm{~Hz}, 2 \mathrm{H}), 7.21-7.09(\mathrm{~m}, 6 \mathrm{H}), 3.91(\mathrm{~s}, 3 \mathrm{H} ; \mathrm{OMe}), 2.36$ (s, 3H; $\left.\mathrm{CH}_{3}\right) ;{ }^{13} \mathrm{CNMR}\left(100 \mathrm{MHz}, \mathrm{CDCl}_{3}\right) \delta 157.7$ (C), 137.4 (C), 134.7 (C), 134.1 (C), 132.9 (C), $129.5(\mathrm{CH}), 129.4\left(\mathrm{CH}_{2} \times 2\right), 129.1(\mathrm{C}), 127.9(\mathrm{CH} \times 2), 127.1(\mathrm{CH}), 126.3(\mathrm{CH} \times 2)$, 126.2 (CH), $124.1(\mathrm{CH}), 119.0(\mathrm{CH}), 105.9(\mathrm{CH}), 55.3\left(\mathrm{CH}_{3}\right), 21.3\left(\mathrm{CH}_{3}\right)$. Spectral data of 
(E)-3z matched reported literature values. ${ }^{12}$

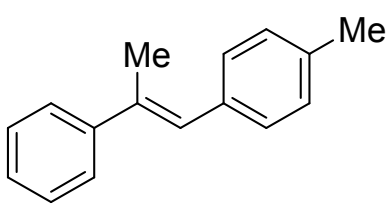

(E)-3aa

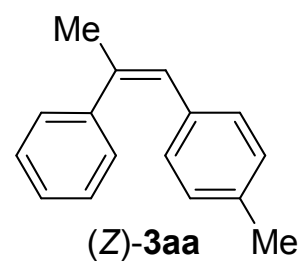

(E)- and (Z)-1-Methyl-4-(2-phenylprop-1-enyl)benzene (3aa). ${ }^{25,19}$ Compounds $(E)$ and (Z)-3aa were synthesized from 1-(1-methoxyprop-1-en-2-yl)benzene 1m $(E / Z=$ $55: 45,0.338 \mathrm{mmol}, 50 \mathrm{mg}$ ) and 5,5-dimethyl-2-p-tolyl-1,3,2-dioxaborinane 2a (0.405 $\mathrm{mmol}, 83 \mathrm{mg}$ ) according to general procedure with $\mathrm{Ni}(\mathrm{acac})_{2}(20 \mathrm{~mol} \%, 0.067 \mathrm{mmol}$, $17 \mathrm{mg}$ ), $\mathrm{PCy}_{3}(60 \mathrm{~mol} \%, 0.203 \mathrm{mmol}, 57 \mathrm{mg}$ ) and diisobutylaluminum hydride (DIBAL, $40 \mathrm{~mol} \%, 1.0 \mathrm{M}$ in hexane, $0.135 \mathrm{mmol}$ ) at $105^{\circ} \mathrm{C}$. Standard work-up afforded crude 3aa as a mixture of $(E)$ - and $(Z)-3 a a$ in a ratio $E / Z=68: 32$, and then purification by column chromatography on silica gel (eluent, petroleum ether/ toluene $=10: 1$ ) yielded the title compound 3aa as a white solid (51 mg, $0.245 \mathrm{mmol}, 72 \%$ yield). As $(E)$ - and (Z)-3aa are inseparable, NMR data are given for the mixture of the two isomers. (E)-3aa: ${ }^{1} \mathrm{H}$ NMR $\left(400 \mathrm{MHz}, \mathrm{CDCl}_{3}\right) \delta 7.52-7.50(\mathrm{~m}, 2 \mathrm{H} ; \mathrm{Ar}-\mathrm{H}), 7.38-7.34(\mathrm{~m}$, 2H; Ar-H), 7.29-7.24 (m, 3H; Ar-H), 7.18 (d, J = 8.0 Hz, 2H; Ar-H), 6.80 (s, 1H; olefin-H), $2.36\left(\mathrm{~s}, 3 \mathrm{H} ; \mathrm{CH}_{3}\right), 2.27\left(\mathrm{~d}, J=1.3 \mathrm{~Hz}, 3 \mathrm{H} ; \mathrm{CH}_{3}\right) ;{ }^{13} \mathrm{C} \mathrm{NMR}\left(100 \mathrm{MHz}, \mathrm{CDCl}_{3}\right) \delta 144.1(\mathrm{C})$, 136.7 (C), 136.2 (C), 135.4 (C), $129.1(\mathrm{CH} \times 2), 128.9(\mathrm{CH} \times 2), 128.3(\mathrm{CH} \times 2), 127.6$ $(\mathrm{CH}), 127.0(\mathrm{CH}), 126.0(\mathrm{CH} \times 2), 21.2\left(\mathrm{CH}_{3}\right), 17.5\left(\mathrm{CH}_{3}\right)$. Spectral data of $(E)$-3aa matched reported literature values. ${ }^{25}(Z)-3 a a:{ }^{1} \mathrm{H}$ NMR $\left(400 \mathrm{MHz}, \mathrm{CDCl}_{3}\right) \delta 7.33-7.15$ (m, 5H; Ar-H), 6.89 (d, J = 8.3 Hz, 2H; Ar-H), 6.82 (d, J = $8.3 \mathrm{~Hz}, 2 \mathrm{H} ; \operatorname{Ar}-\mathrm{H}), 6.43$ (s, $1 \mathrm{H}$; olefin- $\mathrm{H}), 2.23\left(\mathrm{~s}, 3 \mathrm{H} ; \mathrm{CH}_{3}\right), 2.18\left(\mathrm{~d}, J=1.5 \mathrm{~Hz}, 3 \mathrm{H} ; \mathrm{CH}_{3}\right) ;{ }^{13} \mathrm{C} \mathrm{NMR}\left(100 \mathrm{MHz}, \mathrm{CDCl}_{3}\right) \delta$ $142.3(\mathrm{C}), 137.8(\mathrm{C}), 135.7$ (C), $134.7(\mathrm{C}), 128.8(\mathrm{CH} \times 2), 128.6(\mathrm{CH} \times 2), 128.4(\mathrm{CH} \times 2)$, 128.2 $(\mathrm{CH} \times 2), 126.8(\mathrm{CH}), 126.4(\mathrm{CH}), 27.1\left(\mathrm{CH}_{3}\right), 21.1\left(\mathrm{CH}_{3}\right)$. Spectral data of $(\mathrm{Z})-3 a a$ 
matched reported literature values. ${ }^{19}$<smiles>COc1ccc(/C=C/c2cc(OC)c(OC)c(OC)c2)cc1</smiles>

DMU-212 (E-3ab). ${ }^{7}$ Compound (E)-3ab was synthesized from 1,2,3-trimethoxy-5-(2methoxyvinyl)benzene 1n ( $E / Z=55: 45,0.272 \mathrm{mmol}, 61 \mathrm{mg}$ ) and 2-(4-methoxyphenyl)5,5-dimethyl-1,3,2-dioxaborinane $2 \mathrm{~h}(0.326 \mathrm{mmol}, 72 \mathrm{mg})$ according to general procedure at $105^{\circ} \mathrm{C}$. Standard work-up afforded crude product containing the $(E)-3 a b$ as a single diastereomer (as determined by ${ }^{1} \mathrm{H} N \mathrm{NM}$ ), and then purification by column chromatography on silica gel (eluent, petroleum ether/ ethyl acetata $=5: 1$ ) yielded (E)-3ab as a a beige solid (66 mg, $0.220 \mathrm{mmol}, 81 \%$ yield). DMU-212: ${ }^{1} \mathrm{H}$ NMR (400 $\left.\mathrm{MHz}, \mathrm{CDCl}_{3}\right) \delta 7.44-7.41(\mathrm{~m}, 2 \mathrm{H}), 6.95(\mathrm{~d}, J=16.2 \mathrm{~Hz}, 1 \mathrm{H}), 6.90-6.89(\mathrm{~m}, 2 \mathrm{H}), 6.87(\mathrm{~d}$, $J=16.2 \mathrm{~Hz}, 1 \mathrm{H}), 6.70(\mathrm{~s}, 2 \mathrm{H}), 3.90(\mathrm{~s}, 6 \mathrm{H}), 3.85(\mathrm{~s}, 3 \mathrm{H}), 3.81(\mathrm{~s}, 3 \mathrm{H}) ;{ }^{13} \mathrm{C}$ NMR $(100 \mathrm{MHz}$, $\left.\mathrm{CDCl}_{3}\right) \delta 159.4(\mathrm{C}), 153.5(\mathrm{C} \times 2), 137.7(\mathrm{C}), 133.6(\mathrm{C}), 130.1(\mathrm{C}), 127.9(\mathrm{CH}), 127.7(\mathrm{CH}$ $\times 2), 126.7(\mathrm{CH}), 114.3(\mathrm{CH} \times 2), 103.4(\mathrm{CH} \times 2), 61.1\left(\mathrm{CH}_{3}\right), 56.2\left(\mathrm{CH}_{3} \times 2\right), 55.4\left(\mathrm{CH}_{3}\right)$. Spectral data of DMU-212 matched reported literature values. ${ }^{7}$

\section{Unsuccessful Substrates}

Boronic ester derivatives<smiles>Cc1cc(C)c(B2OCC(C)(C)CO2)c(C)c1</smiles><smiles>CC1(C)COB(c2cccc([N+](=O)[O-])c2)OC1</smiles>

Alkenyl ether derivatives<smiles>CC1(C)COB(c2cccnc2)OC1</smiles><smiles>C1=COCCC1</smiles><smiles>c1ccc2occc2c1</smiles><smiles>CC1(C)COB(c2ccsc2)OC1</smiles><smiles>CC1(C)COB(c2ccc(Cl)cc2)OC1</smiles><smiles>COC=CCCCCC(=O)OC</smiles> 


\section{Scale-up experiment}

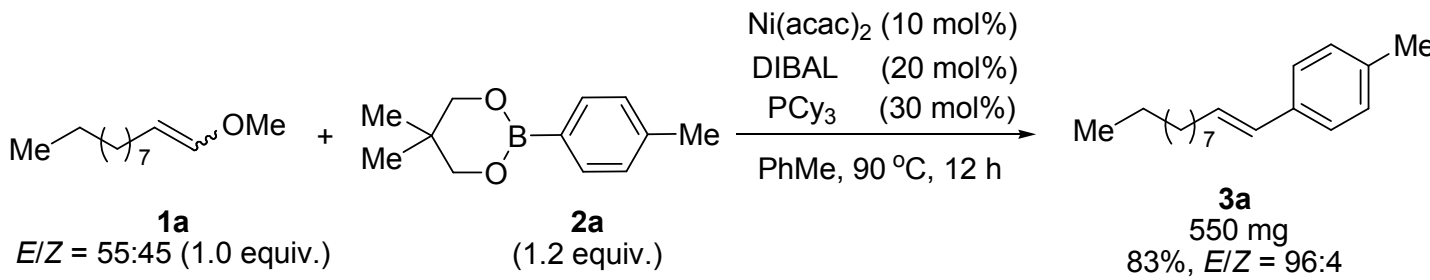

To a flame-dried Schlenk flask equipped with a rubber septum and magnetic stir bar was purged with argon, then $\mathrm{Ni}(\mathrm{acac})_{2}(10 \mathrm{~mol} \%, 0.272 \mathrm{mmol}, 70 \mathrm{mg})$ and $\mathrm{PC}_{3}(30$ mol\%, $0.815 \mathrm{mmol}, 229 \mathrm{mg}$ ) were added. The flask was evacuated and backfilled with argon ( 3 times), and then toluene $(10 \mathrm{~mL})$ was added by a syringe. Diisobutylaluminum hydride (DIBAL, $1.0 \mathrm{M}$ in hexane, $20 \mathrm{~mol} \%, 0.543 \mathrm{mmol}$ ) was then added dropwise with a syringe at ambient temperature over $5 \mathrm{~min}$. After stirring at ambient temperature for $15 \mathrm{~min}$, a solution of 5,5-dimethyl-2-p-tolyl-1,3,2-dioxaborinane $\mathbf{2 a}$ (1.2 equiv., $3.261 \mathrm{mmol}, 666 \mathrm{mg}$ ) in toluene $(4 \mathrm{~mL}$ ) and alkenyl methyl ether 1a (1.0 equiv., $2.717 \mathrm{mmol}, 500 \mathrm{mg}$ ) were added dropwise to the reaction mixture via syringe under argon. The resulting mixture was heated at the $90^{\circ} \mathrm{C}$ for $12 \mathrm{~h}$. After being cooled to ambient temperature, the reaction mixture was quenched with an aqueous saturated solution of $\mathrm{NH}_{4} \mathrm{Cl}$ and diluted with $\mathrm{Et}_{2} \mathrm{O}$. The combined organic phase was filtered through a pad of silica gel with copious washings by $\mathrm{Et}_{2} \mathrm{O}$ and concentrated. The crude mixture was purified by column chromatography on silica gel (eluent, petroleum ether/toluene $=20: 1$ ) to afford isolated yield of the desired styrene $3 a$ as a clear oil (550 mg, $2.254 \mathrm{mmol}, 83 \%$ yield, $E / Z=96: 4)$. 


\section{Control experiments to identify isomerization-active species}

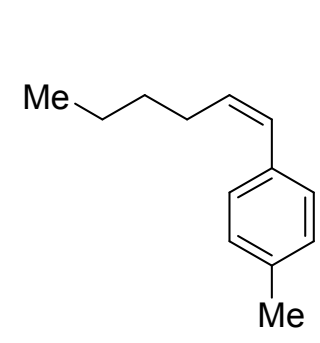

(Z)-3p

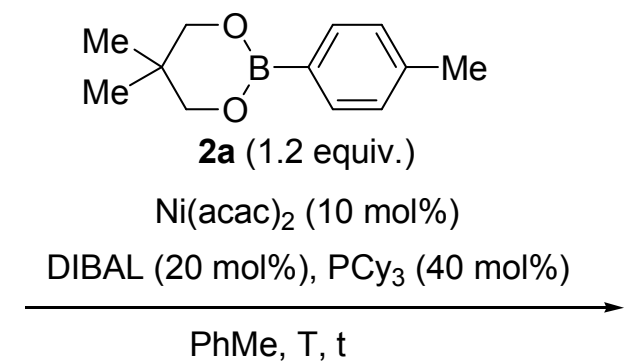

PhMe, T, t

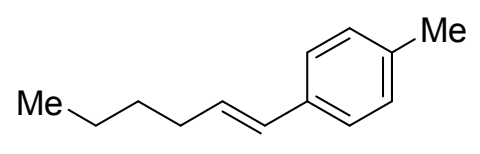

(E)-3p

\begin{tabular}{lllllllll}
\hline Entry $^{\mathrm{a}}$ & $\mathrm{Ni}(\mathrm{acac})_{2}$ & $\mathrm{DIBAL}$ & $\mathrm{PCY}_{3}$ & $\mathbf{2 a}$ & $\mathrm{T}\left({ }^{\circ} \mathrm{C}\right)$ & $\mathrm{t}(\mathrm{h})$ & ${\text { Yield }(\%)^{\mathrm{b}}}$ & $E / Z^{\mathrm{c}}$ \\
\hline 1 & $\checkmark$ & $\checkmark$ & $\checkmark$ & $\checkmark$ & 105 & 16 & 66 & $98: 2$ \\
2 & $\checkmark$ & $\checkmark$ & $\checkmark$ & $\mathbf{x}$ & 105 & 16 & 74 & $96: 4$ \\
3 & $\checkmark$ & $\checkmark$ & $\mathbf{x}$ & $\mathbf{x}$ & 105 & 16 & 63 & $95: 5$ \\
4 & $\checkmark$ & $\mathbf{x}$ & $\mathbf{x}$ & $\mathbf{x}$ & 105 & 16 & n.r. & n.r. \\
5 & $\checkmark$ & $\checkmark$ & $\mathbf{x}$ & $\mathbf{x}$ & 60 & 16 & 74 & $>95: 1$ \\
6 & $\checkmark$ & $\checkmark$ & $\mathbf{x}$ & $\mathbf{x}$ & 25 & 48 & 81 & $>95: 1$ \\
\hline
\end{tabular}

${ }^{a}$ All reactions were carried out using (Z)-3p (0.287 mmol) and, if applicable, 2a (0.345 mmol, $\left.120 \mathrm{~mol} \%\right)$ in $1.4 \mathrm{~mL}$ toluene. ${ }^{b}$ Yields determined by analysis of the unpurified mixture of products by ${ }^{1} \mathrm{H}$ NMR with an internal standard in chloroform-D. ${ }^{C}$ The $E / Z$ ratio was determined by ${ }^{1} \mathrm{H}-\mathrm{NMR}$ spectroscopic analysis of the crude mixture.

General procedure for the control experiments to identify isomerization-active species. To a flame-dried Schlenk flask equipped with a rubber septum and magnetic stir bar was purged with argon, then $\mathrm{Ni}(\mathrm{acac})_{2}(10 \mathrm{~mol} \%, 0.029 \mathrm{mmol}, 7 \mathrm{mg})$ was added, and for entries 1 and 2, $\mathrm{PCY}_{3}$ (40 mol\%, $0.115 \mathrm{mmol}, 32 \mathrm{mg}$ ) was added as well. The flask was put under vacuum and backfilled with argon (3 times), and then toluene (1.4 $\mathrm{mL}$ ) was added by a syringe. For entries 1-3, 5 and 6, Diisobutylaluminum hydride (DIBAL, $20 \mathrm{~mol} \%, 1.0 \mathrm{M}$ in hexane, $0.057 \mathrm{mmol}$ ) was then added dropwise with a syringe at ambient temperature over 2 min. After stirring at ambient temperature for 
$15 \mathrm{~min}$, styrene (Z)-3p (1.0 equiv., $0.287 \mathrm{mmol}, 50 \mathrm{mg}$ ) was added dropwise to the reaction mixture via syringe under argon. For entry 1 , a solution of 5,5-dimethyl-2-ptolyl-1,3,2-dioxaborinane 2 a (1.2 equiv., $0.345 \mathrm{mmol}, 70 \mathrm{mg}$ ) in toluene $(0.4 \mathrm{~mL}$ ) was added to the reaction mixture via syringe under argon. The reaction mixture was then stirred at the indicated temperature for the specified time. At that point, the resulting mixture for entries 1-5 were cooled to ambient temperature. The reaction mixture was then quenched with an aqueous saturated solution of $\mathrm{NH}_{4} \mathrm{Cl}$ and diluted with $\mathrm{Et}_{2} \mathrm{O}$. The combined organic phase was filtered through a pad of silica gel with copious washings by $\mathrm{Et}_{2} \mathrm{O}$ and concentrated. 2-Naphthaldehyde was then added as an internal standard and a small aliquot in chloroform- $d_{1}$ was used for ${ }^{1} \mathrm{H}$ NMR analysis to evaluate the yield and $E / Z$ ration of styrene $3 p .{ }^{1} \mathrm{H}$ and ${ }^{13} \mathrm{C}$ NMR spectra for both $(Z)-$ and $(E)-3 p$ have been shown in the description above. The crude ${ }^{1} \mathrm{H}$ NMR spectra for entries 1-6 are shown below.

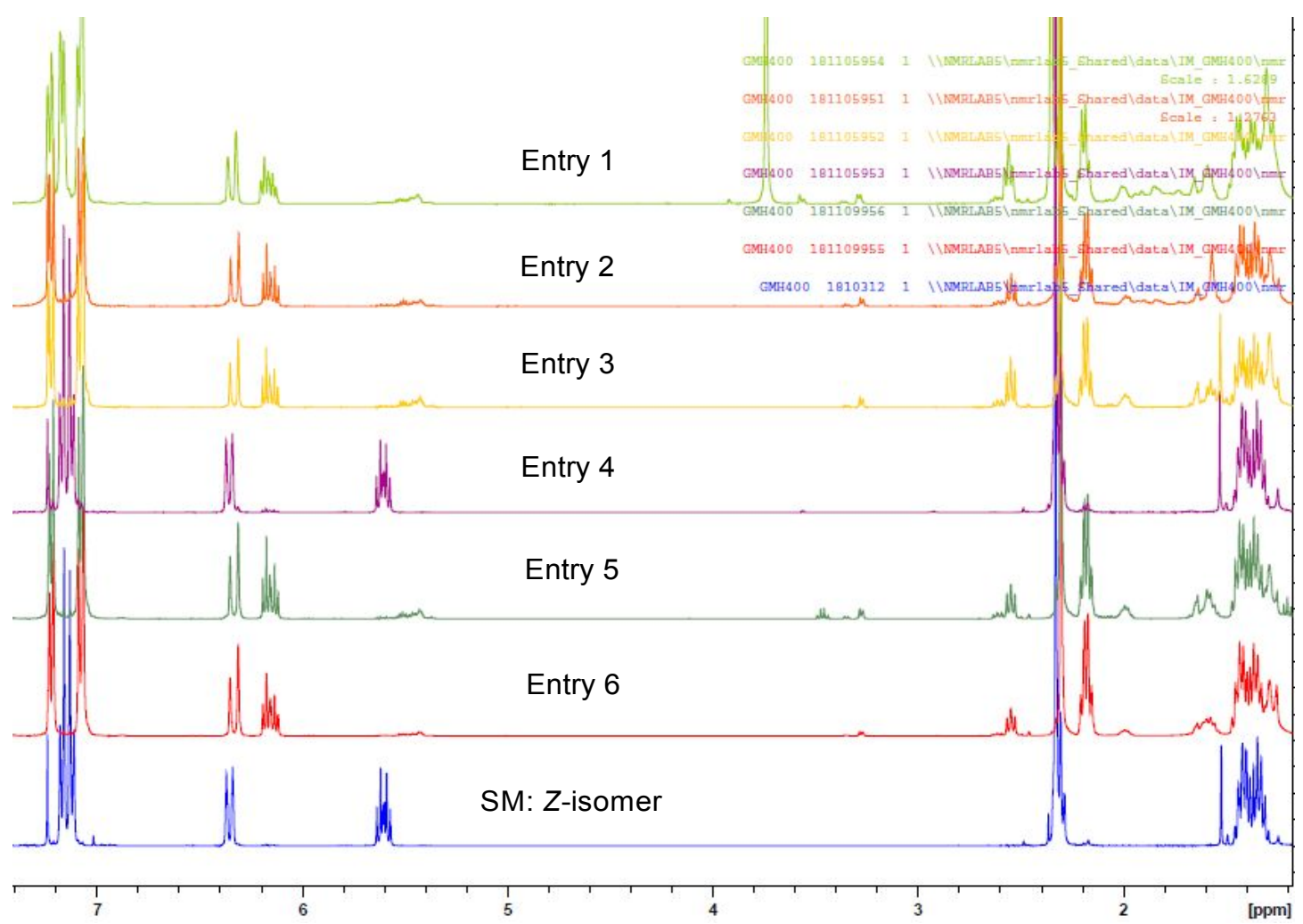




\section{References}

1. Y. Koseki, K. Kitazawa, M. Miyake, T. Kochi, F. Kakiuchi, J. Org. Chem. 2017, 82, 6503-6510.

2. T. L. Andersen, M. W. Frederiksen, K. Domino, T. Skrydstrup, Angew. Chem. Int. Ed. 2016, 55, $10396-10400$.

3. X.-Y. Lu, C.-T. Yang, J.-H. Liu, Z.-Q. Zhang, X. Lu, X. Lou, B. Xiao, Y. Fu, Chem. Commun., 2015, 51, 2388-2391.

4. Y. Zhao, V. Snieckus, J. Am. Chem. Soc. 2014, 136, 11224-11227.

5. M. Takeda, K. Takatsu, R. Shintani, T. Hayashi, J. Org. Chem. 2014, 79, 2354-2367.

6. Y.-Y. Sun, J. Yi, X. Lu, Z.-Q. Zhang, B. Xiao, Y. Fu, Chem. Commun., 2014, 50, 11060-11062.

7. T. Hostier, Z. Neouchy, V. Ferey, D. G. Pardo, J. Cossy, Org. Lett., 2018, 20, 1815-1818.

8. K. A. Ogawa, A. E. Goetz, A. J. Boydston, J. Am. Chem. Soc. 2015, 137, 1400-1403.

9. T. Yoshimitsu, R. Nakatani, A. Kobayashi, T. Tanaka, Org. Lett., 2011, 13, 908-901.

10. M. G. Lauer, W. H. Henderson, A. Awad, J. P. Stambuli, Org. Lett., 2012, 14, 6000-6003.

11. Z. Hou, I. Nakanishi, T. Kinoshita, Y. Takei, M. Yasue, R. Misu, Y. Suzuki, S. Nakamura, T. Kure, H. Ohno, K. Murata, K. Kitaura, A. Hirasawa, G. Tsujimoto, S. Oishi, N. Fujii, J. Med. Chem. 2012, 55, 2899-2903.

12. T. Shimasaki, Y. Konno, M. Tobisu, N. Chatani, Org. Lett., 2009, 11, 4890-4892.

13. Z.-Q. Rong, Y. Zhang, R. H. B. Chua, H.-J. Pan, Y. Zhao, J. Am. Chem. Soc. 2015, 137, 4944-4947.

14. A. Ruiz-Olalla, M. A. Würdemann, M. J. Wanner, S. Ingemann, J. H. van Maarseveen, H. Hiemstra, J. Org. Chem. 2015, 80, 5125-5132.

15. H. J. Callot, A. Louati, M. Gross, Bull. Soc. Chim. Fr. 1983, 317-320.

16. G.-M. Ho, L. Judkele, J. Bruffaerts, I. Marek. Angew. Chem. Int. Ed. 2018, 57, 8012-8016.

17. M. Peña-López, M. Ayán-Varela, L. A. Sarandeses, J. P. Sestelo, Chem. Eur. J., 2010, 16, 9905-9909.

18. X.-H. Ouyang, R.-J. Song, B. Liu, J.-H. Li, Adv. Synth. Catal. 2016, 358, 1903-1909.

19. K. Ishizuka, H. Seike, T. Hatakeyama, M. Nakamura, J. Am. Chem. Soc. 2010, 132, 38, 13117-13119.

20. H. Huang, K. Jia, Y. Chen, Angew. Chem. Int. Ed. 2015, 54, 1881-1884.

21. C. Li, Y. Zhang, Q. Sun, T. Gu, H. Peng, W. Tang, J. Am. Chem. Soc. 2016, 138, 10774-10777. 
22. S. Guduguntla, J.-B. Gualtierotti, S. S. Goh, B. L. Feringa, ACS Catal. 2016, 6, 6591-6595.

23. X. Zeng, C. Miao, S. Wang, C. Xia, W. Sun, Chem. Commun. 2013, 49, 2418-2420.

24. Y. Wang, M Jiang, J.-T. Liu, Adv. Synth. Catal. 2016, 358, 1322-1327.

25. J. F. Guastavino, M. E. Buden, R. A. Rossi, J. Org. Chem. 2014, 79, 9104-9111. 


\section{NMR spectra}

${ }^{1} \mathrm{H}$ NMR Spectrum of $2 \mathrm{I}, 400 \mathrm{MHz} \mathrm{CDCl}_{3}$

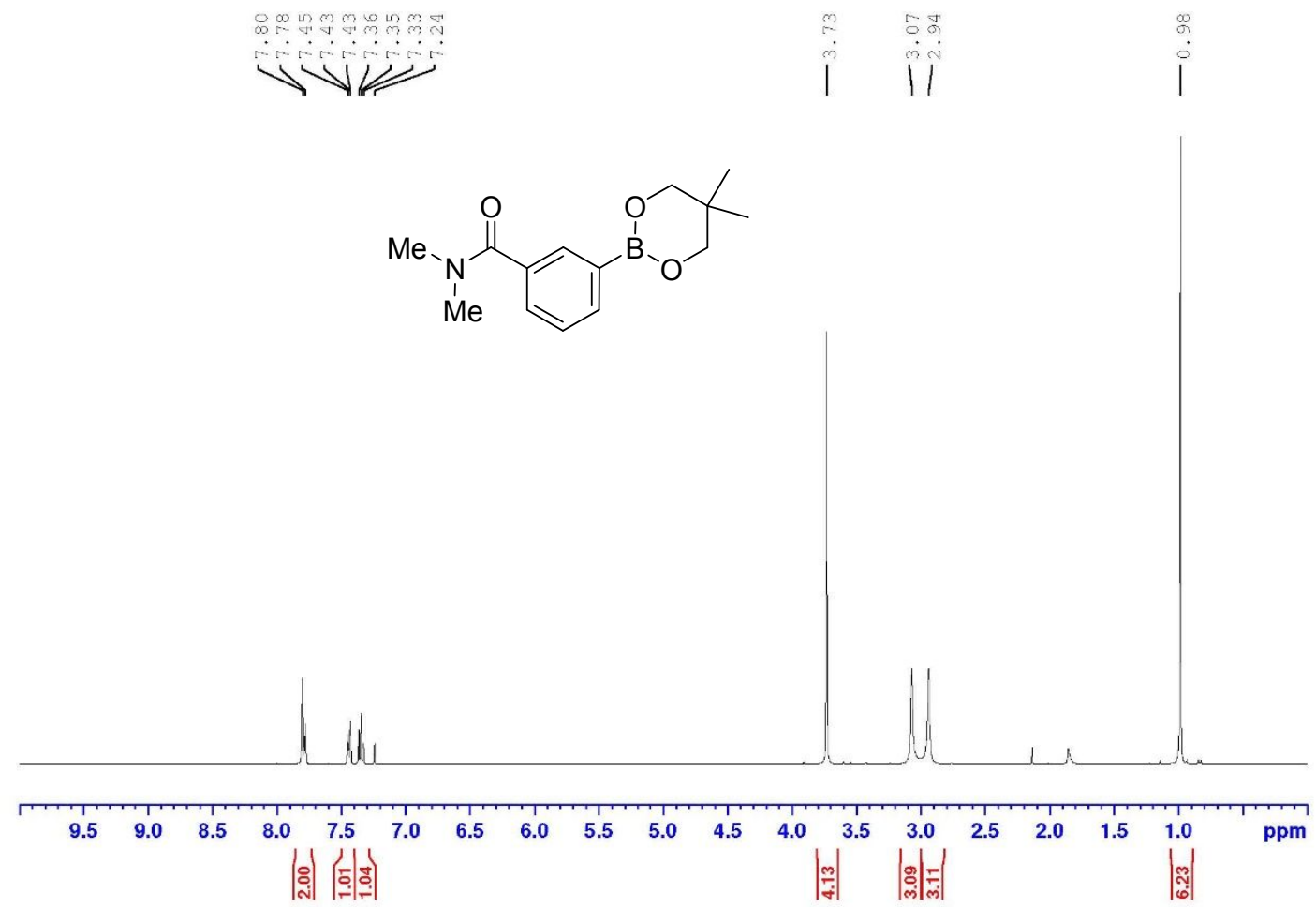

${ }^{13} \mathrm{C}$ NMR Spectrum of $2 \mathrm{l}, 100 \mathrm{MHz}, \mathrm{CDCl}_{3}$ 


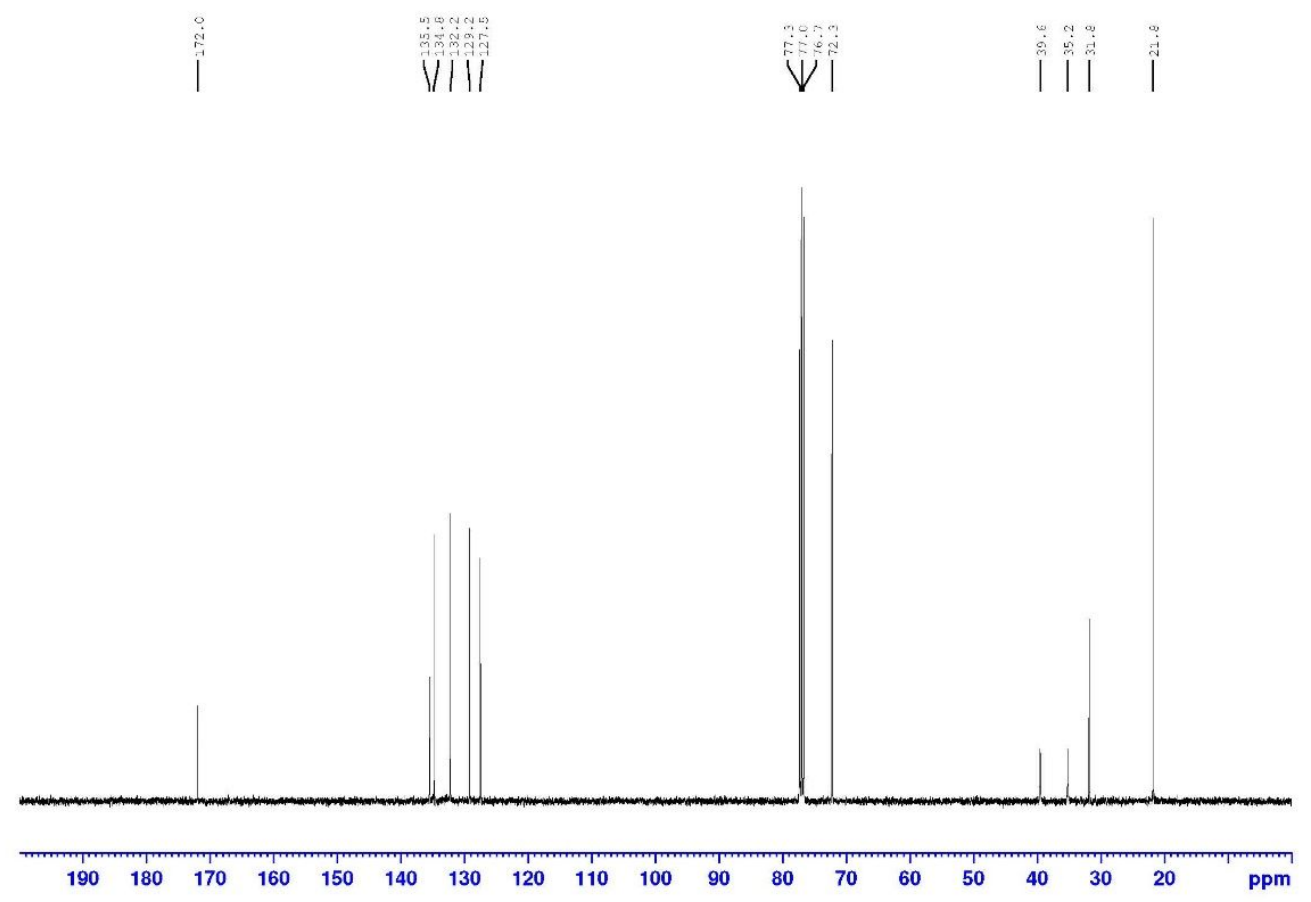

${ }^{1} \mathrm{H}$ NMR Spectrum of $\mathbf{1 b}, 400 \mathrm{MHz}, \mathrm{C}_{6} \mathrm{D}_{6}$

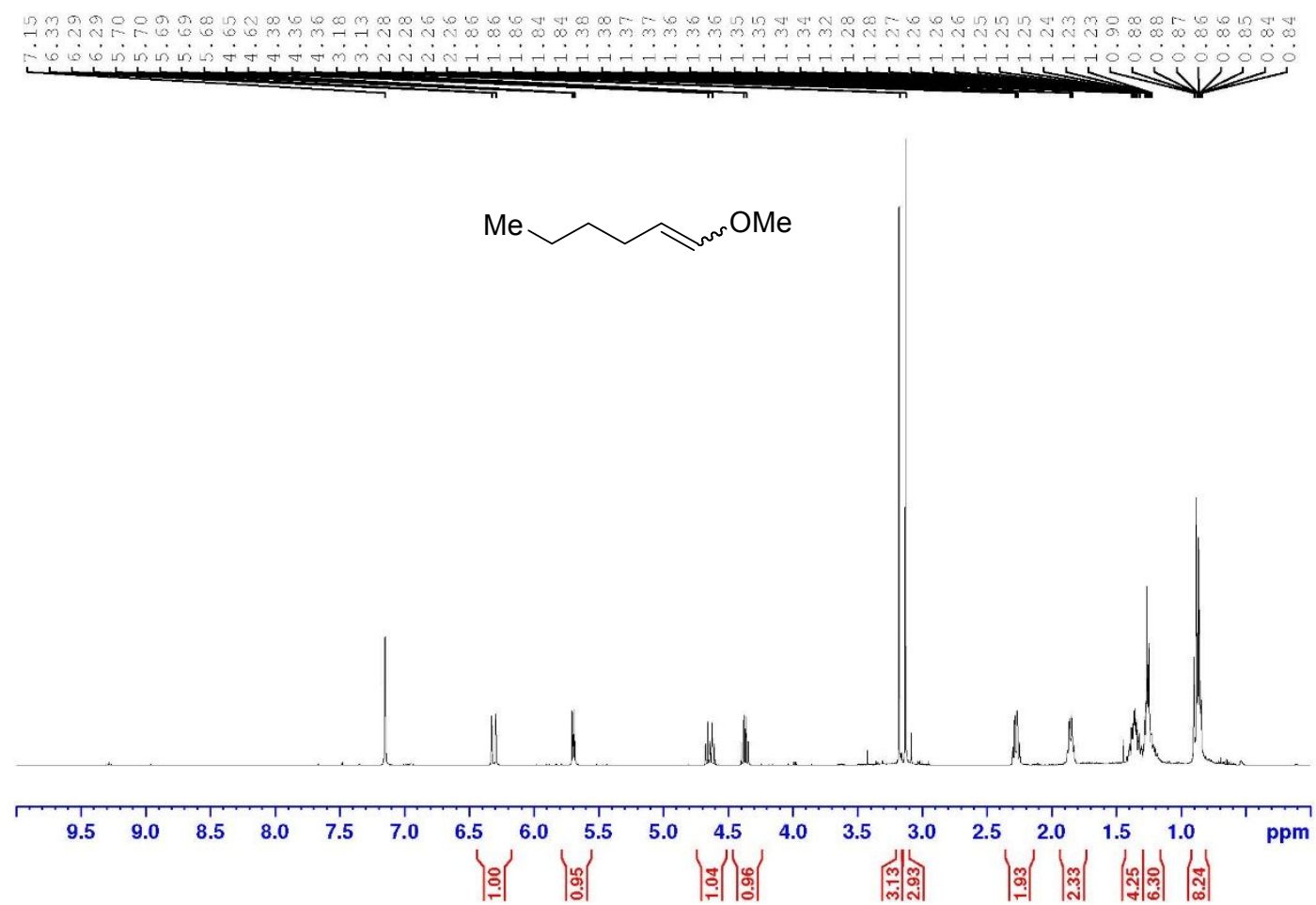

${ }^{13} \mathrm{C}$ NMR Spectrum of $\mathbf{1 b}, 100 \mathrm{MHz}, \mathrm{C}_{6} \mathrm{D}_{6}$ 


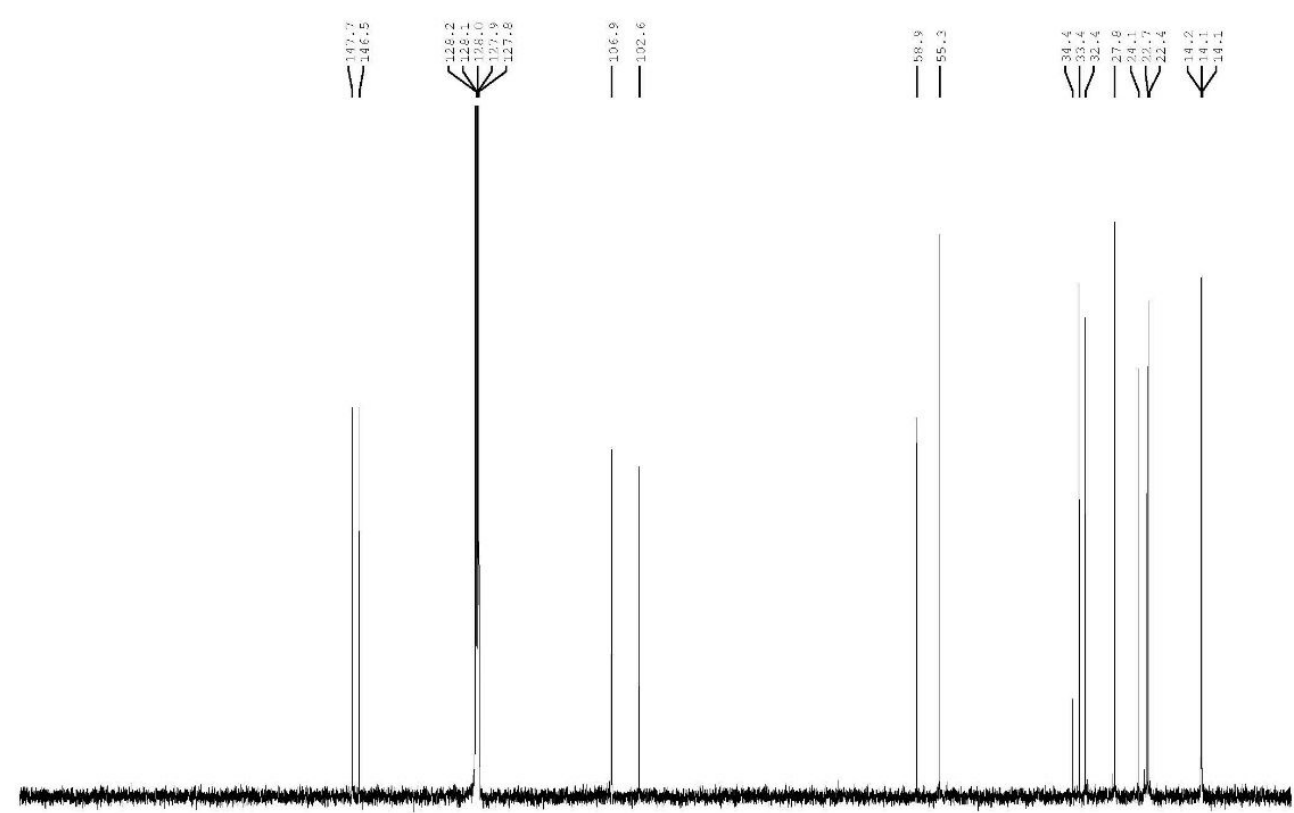

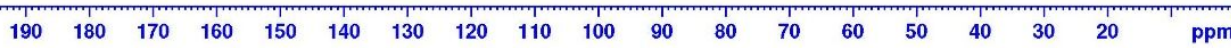

${ }^{1} \mathrm{H}$ NMR Spectrum of $\mathbf{1 g}, 400 \mathrm{MHz}, \mathrm{C}_{6} \mathrm{D}_{6}$

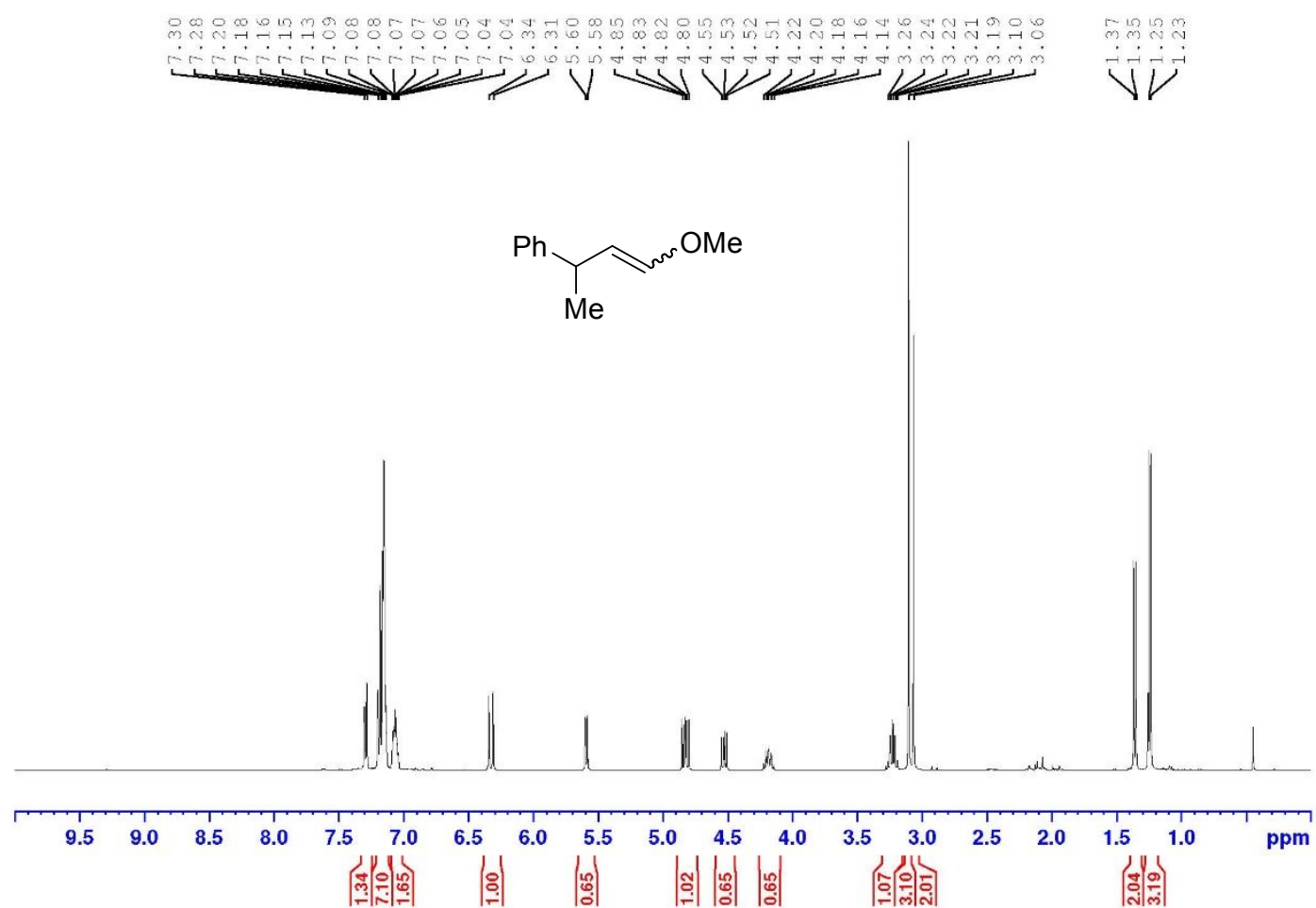

${ }^{13} \mathrm{C}$ NMR Spectrum of $\mathbf{1 g}, 100 \mathrm{MHz}, \mathrm{C}_{6} \mathrm{D}_{6}$ 


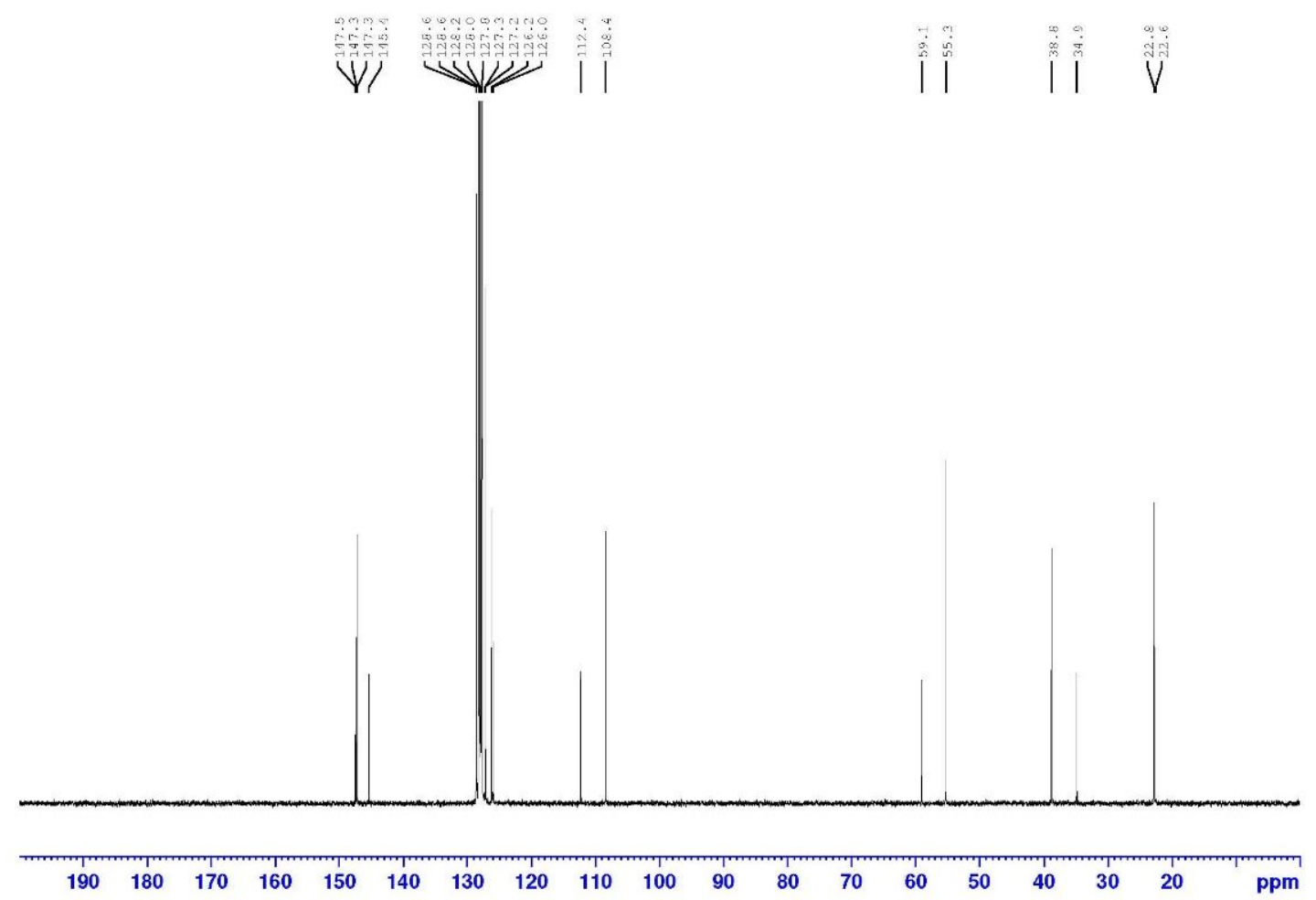

${ }^{1} \mathrm{H}$ NMR Spectrum of $(E)-3 a, 400 \mathrm{MHz}, \mathrm{CDCl}_{3}$

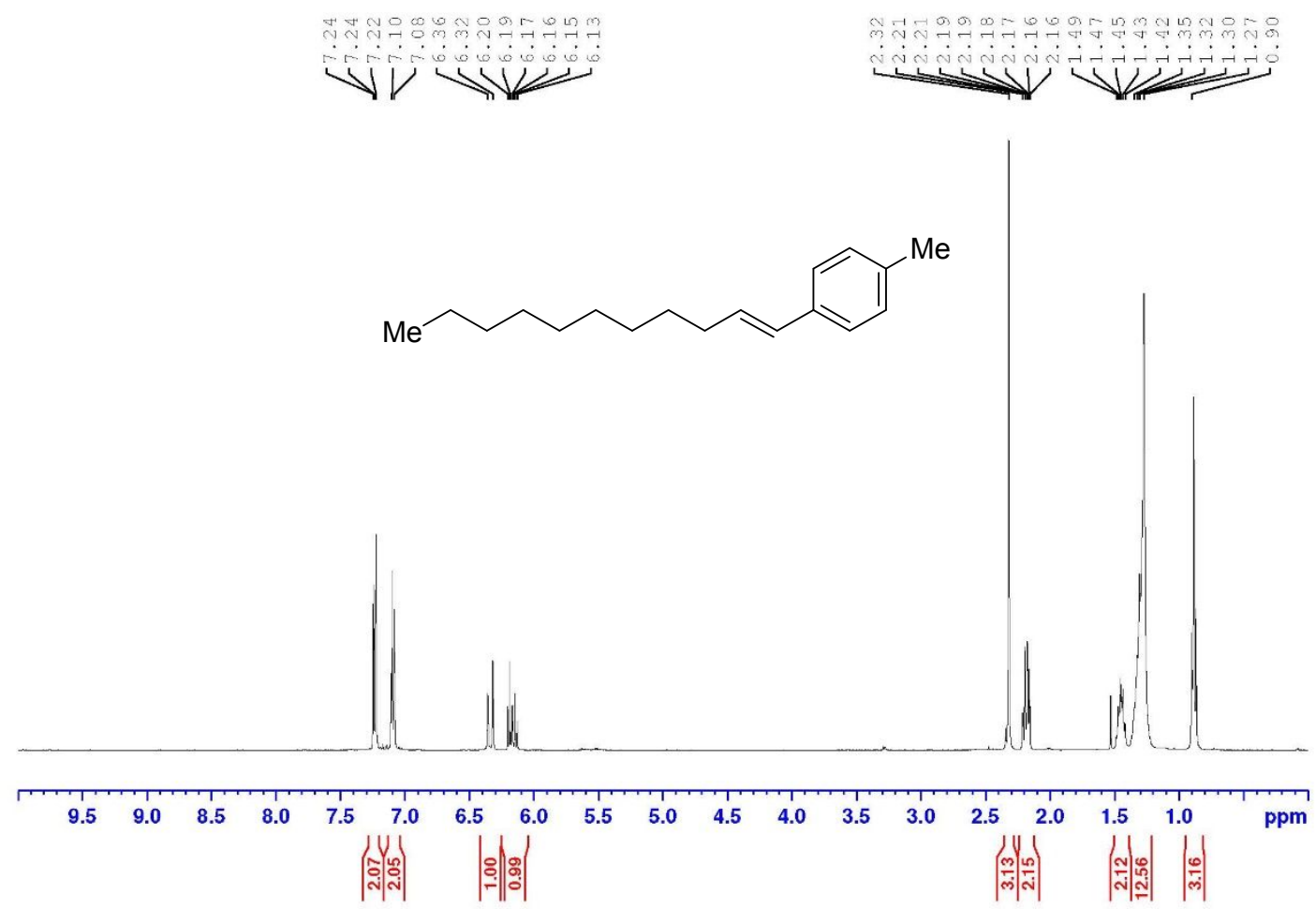

${ }^{13} \mathrm{C}$ NMR Spectrum of (E)-3a, $100 \mathrm{MHz}, \mathrm{CDCl}_{3}$ 


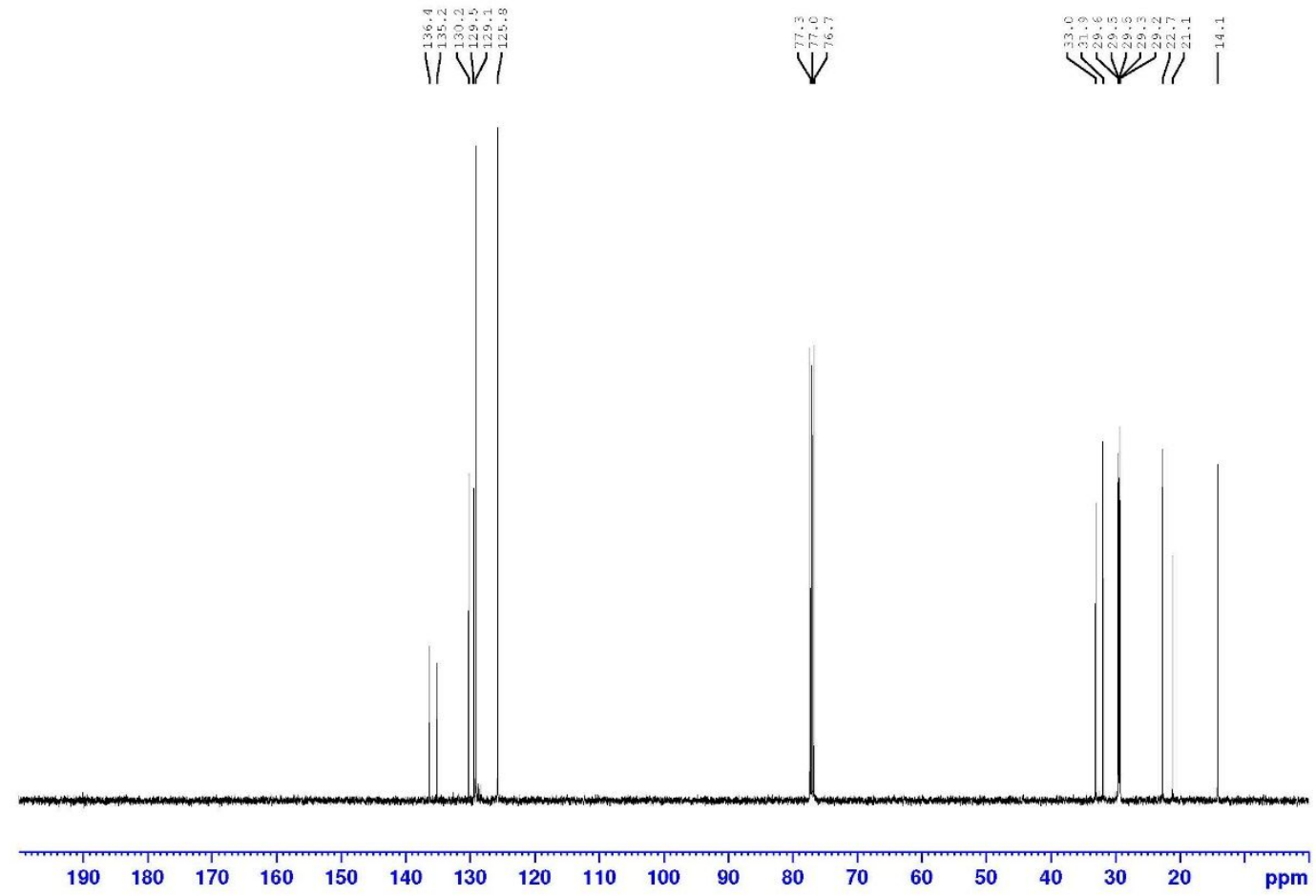

${ }^{1} \mathrm{H}$ NMR Spectrum of (Z)-3a, $400 \mathrm{MHz}, \mathrm{CDCl}_{3}$

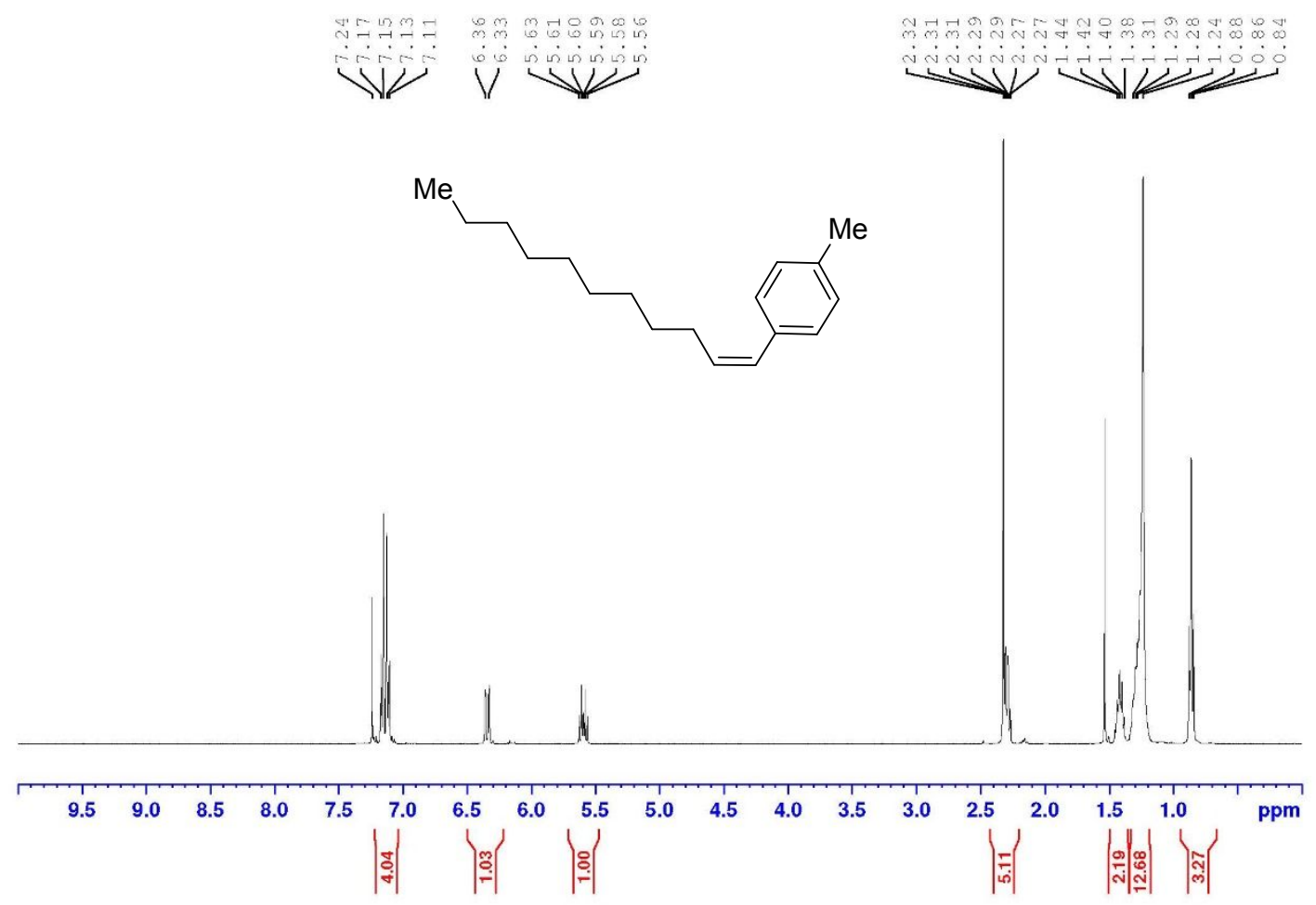

${ }^{13} \mathrm{C}$ NMR Spectrum of (Z)-3a, $100 \mathrm{MHz}, \mathrm{CDCl}_{3}$ 


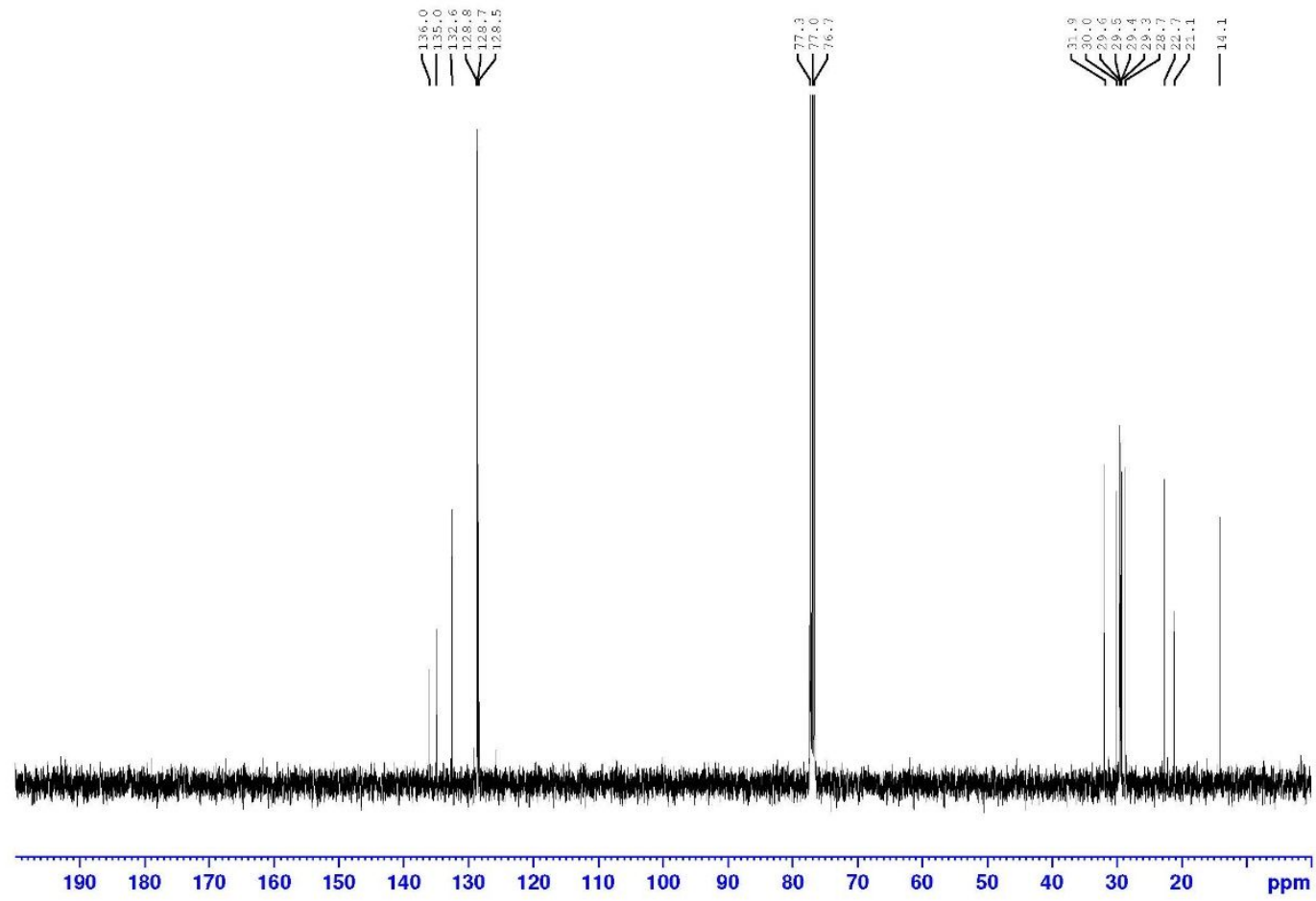

${ }^{1} \mathrm{H}$ NMR Spectrum of $(E)-3 \mathbf{b}, 400 \mathrm{MHz}, \mathrm{CDCl}_{3}$

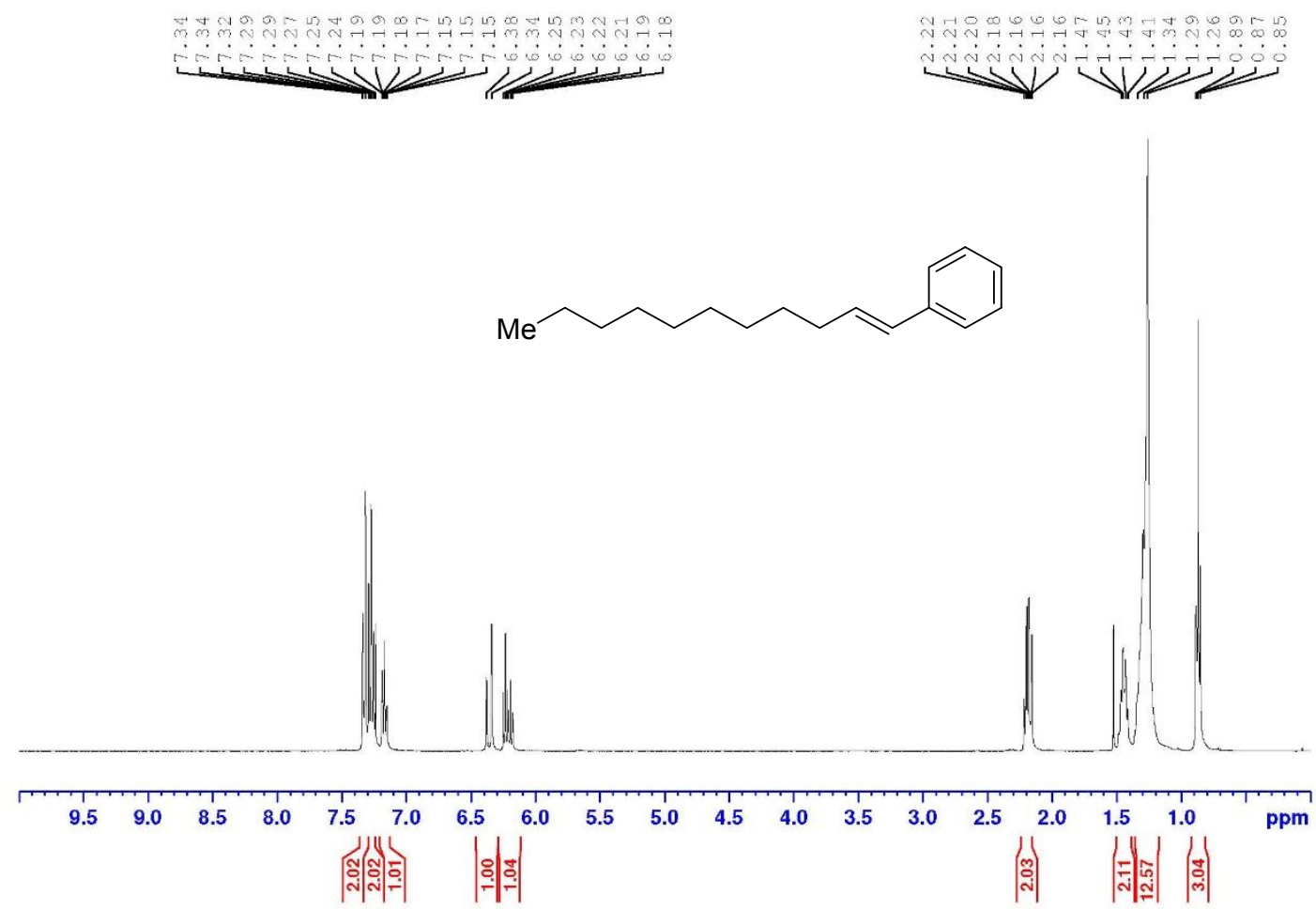

${ }^{13} \mathrm{C}$ NMR Spectrum of (E)-3b, $100 \mathrm{MHz}, \mathrm{CDCl}_{3}$ 


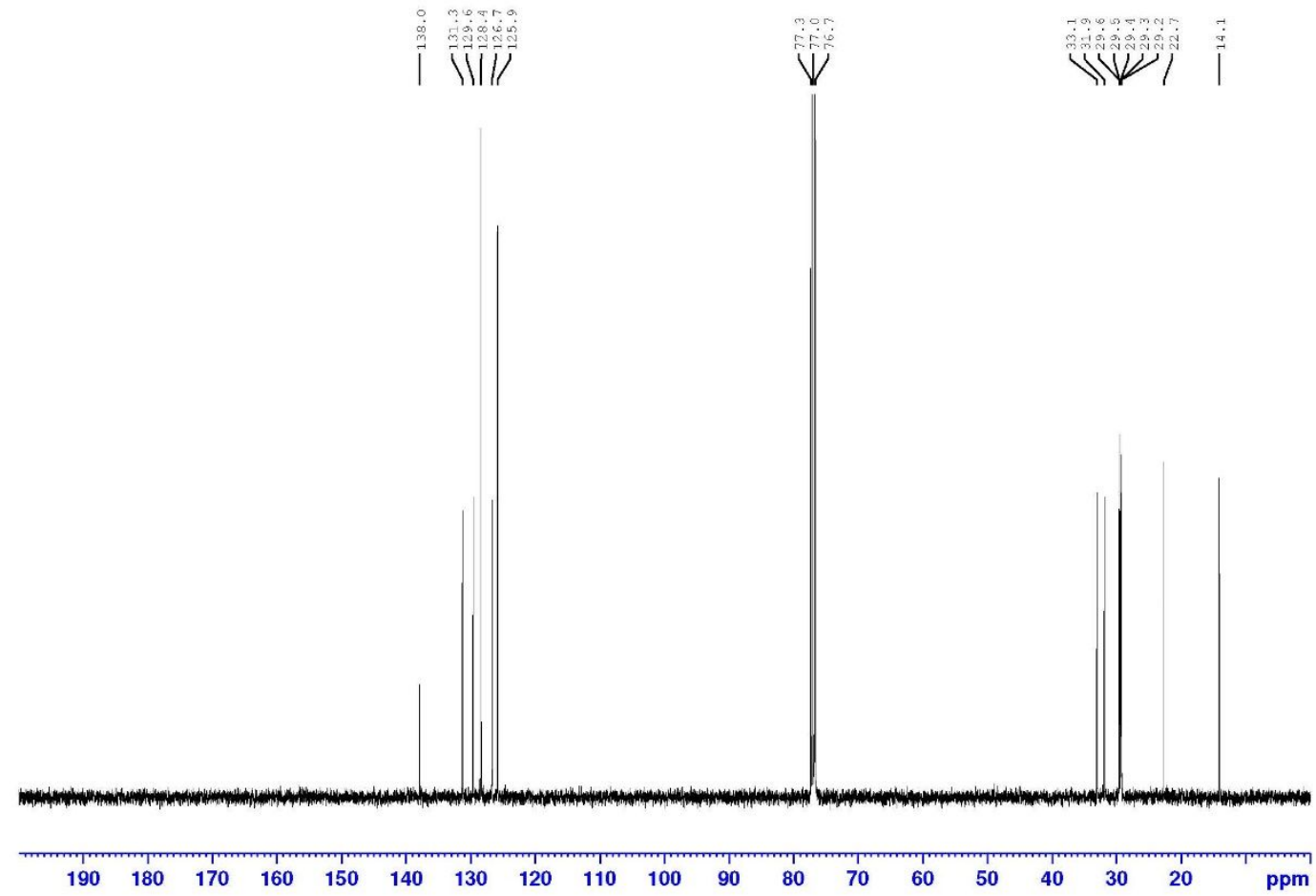

${ }^{1} \mathrm{H}$ NMR Spectrum of (Z)-3b, $400 \mathrm{MHz}, \mathrm{CDCl}_{3}$

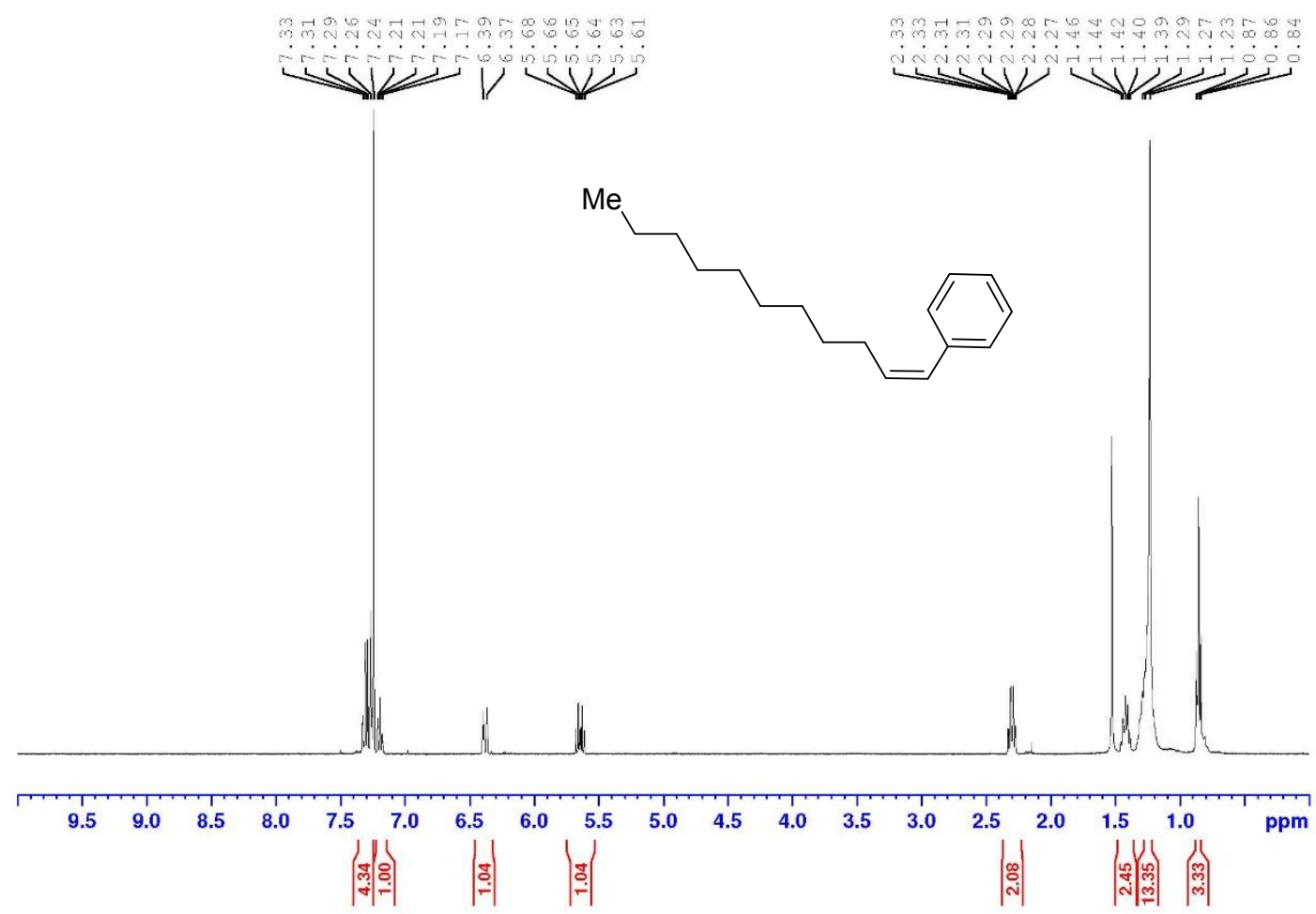

${ }^{13} \mathrm{C}$ NMR Spectrum of (Z)-3b, $100 \mathrm{MHz}, \mathrm{CDCl}_{3}$ 

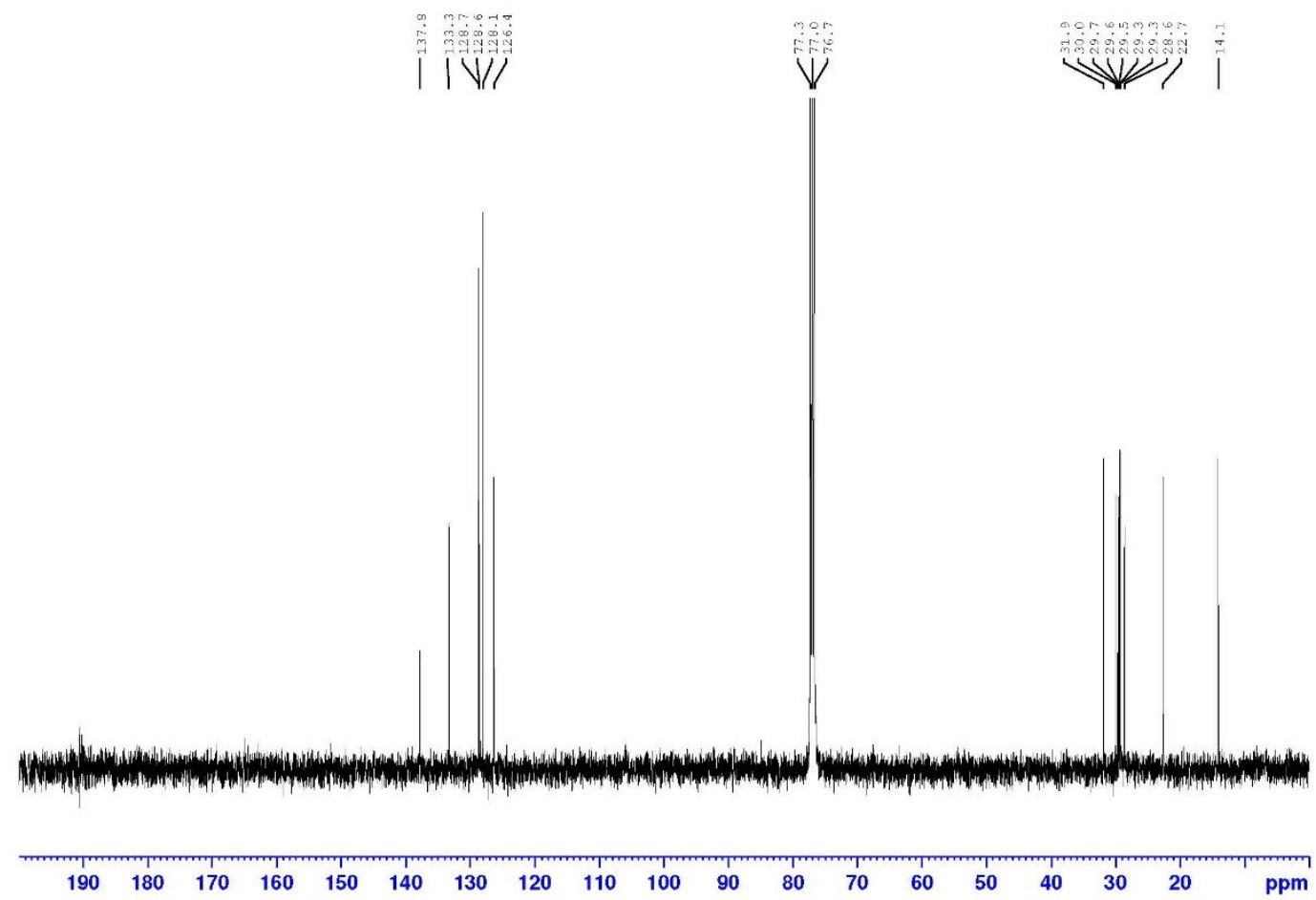

${ }^{1} \mathrm{H}$ NMR Spectrum of $(E)-3 \mathrm{c}, 400 \mathrm{MHz}, \mathrm{CDCl}_{3}$

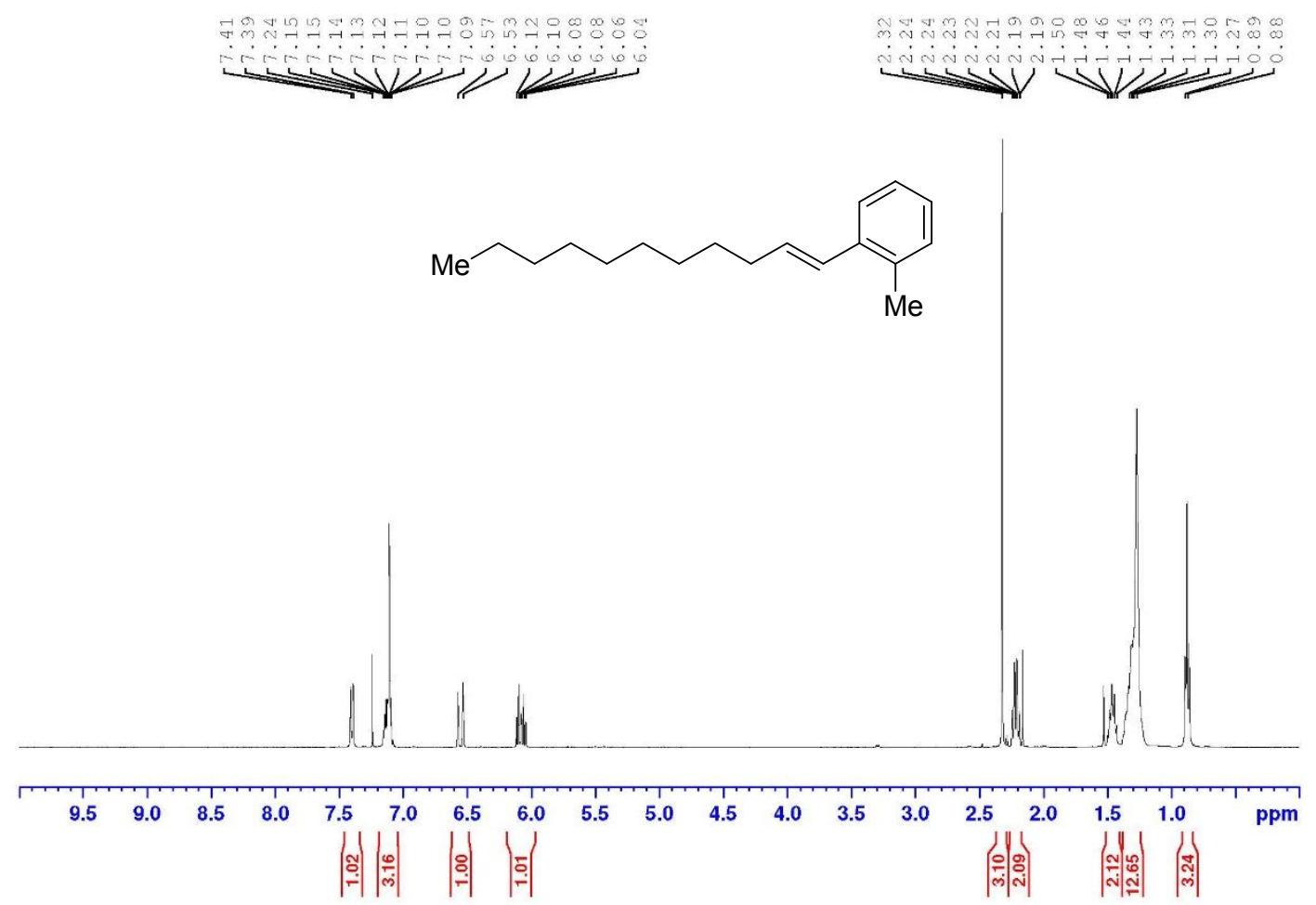

${ }^{13} \mathrm{C}$ NMR Spectrum of (E)-3c, $100 \mathrm{MHz}, \mathrm{CDCl}_{3}$ 


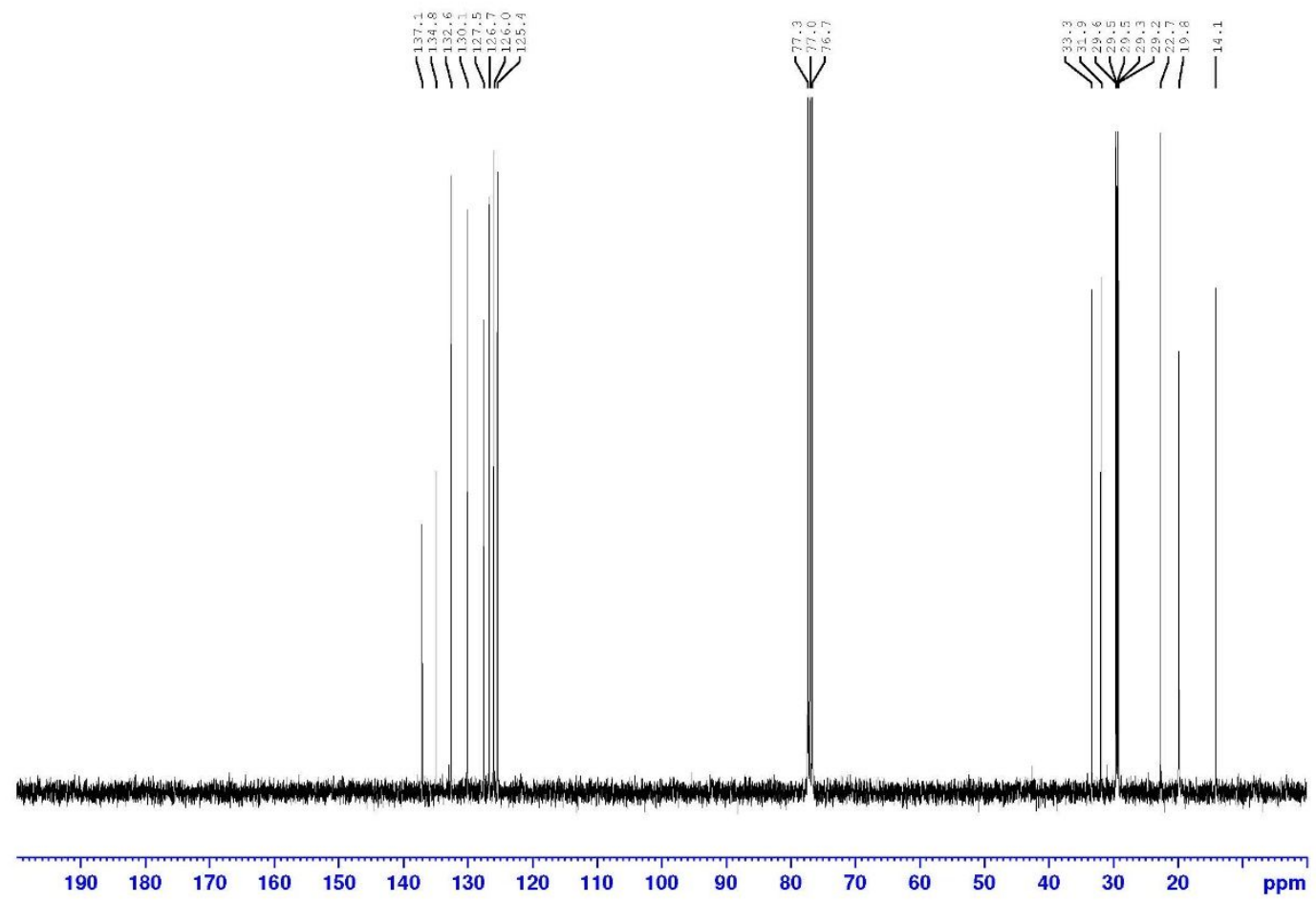

${ }^{1} \mathrm{H}$ NMR Spectrum of (Z)-3c, $400 \mathrm{MHz}, \mathrm{CDCl}_{3}$

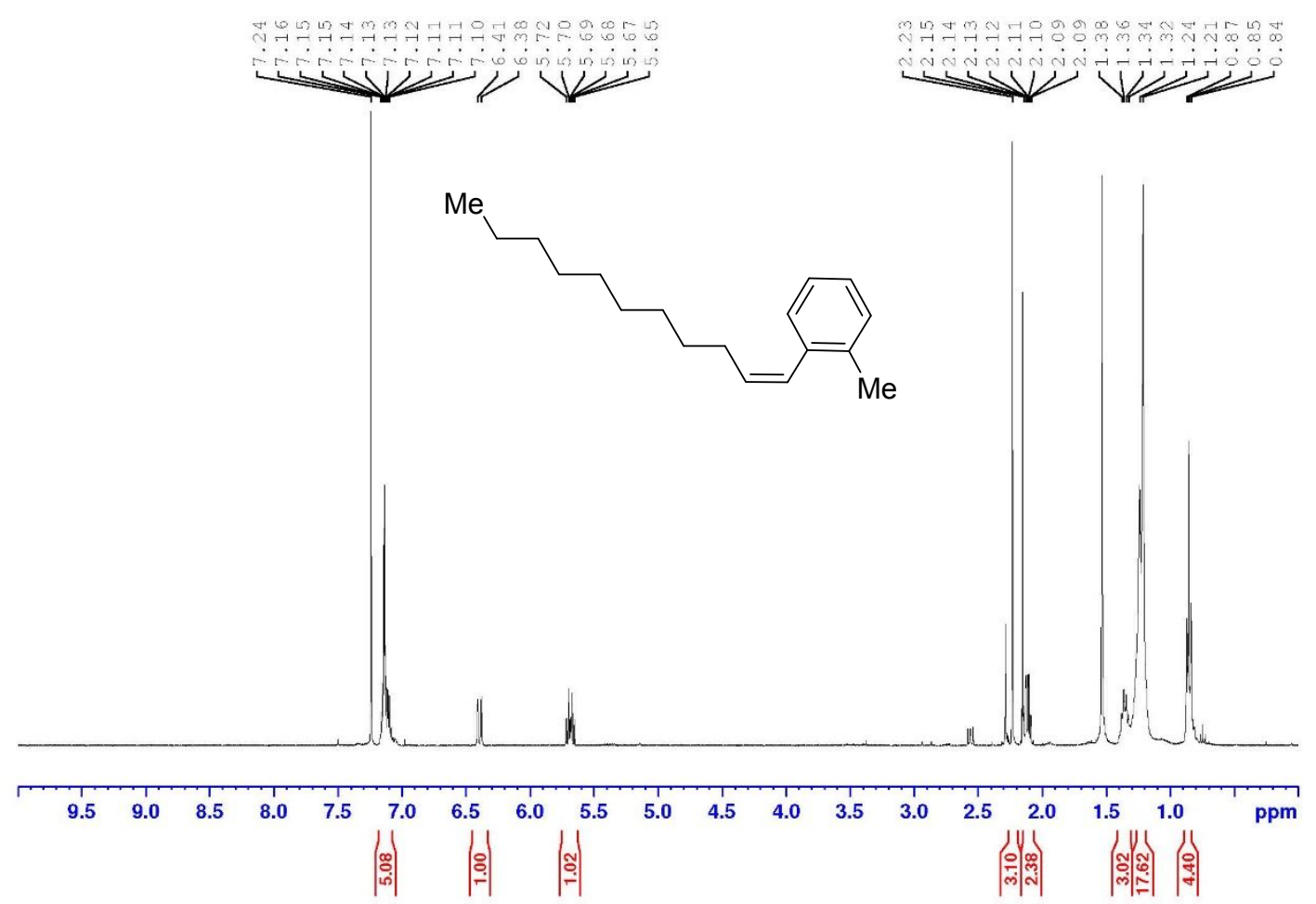

${ }^{13} \mathrm{C}$ NMR Spectrum of (Z)-3c, $100 \mathrm{MHz}, \mathrm{CDCl}_{3}$ 

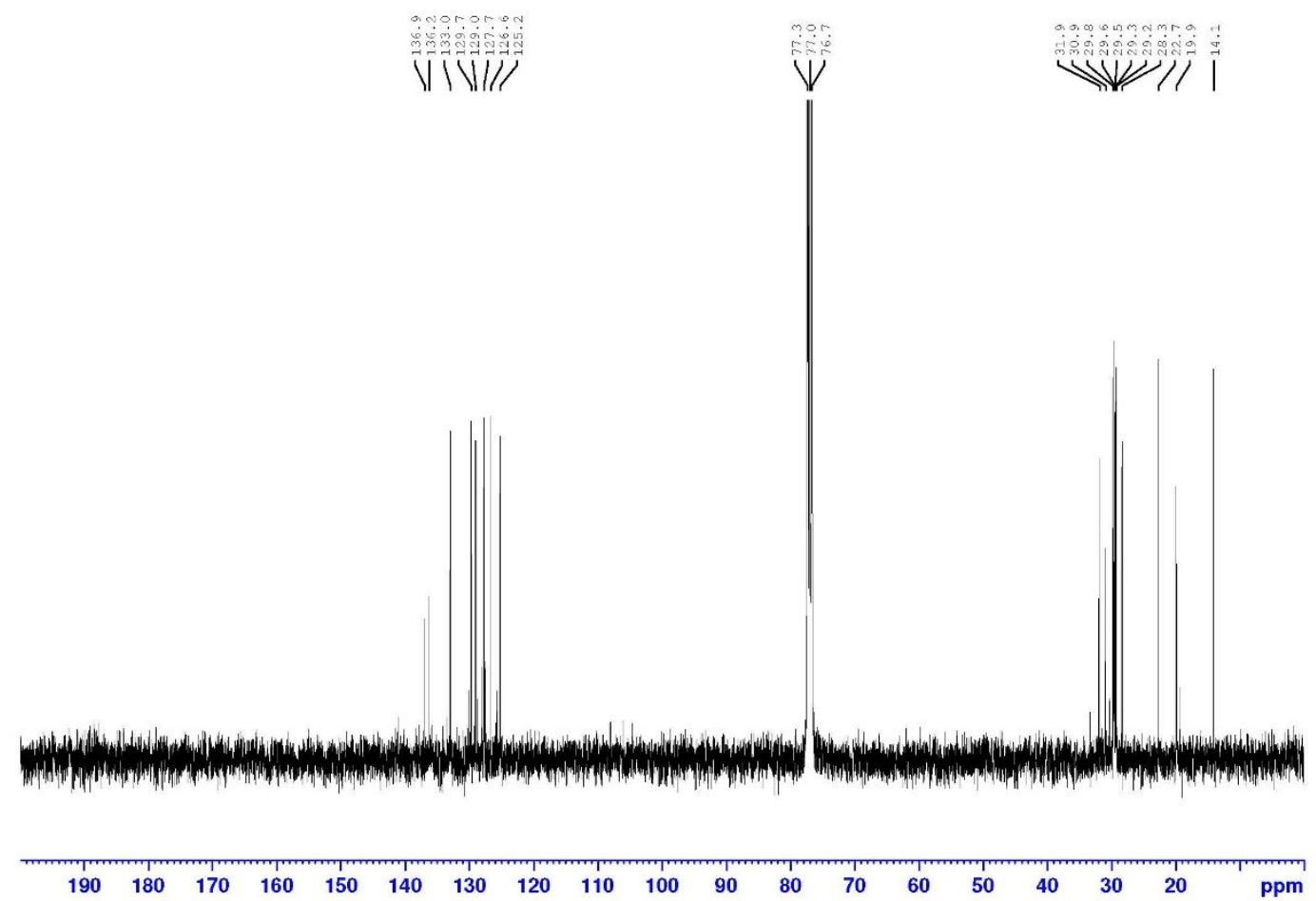

${ }^{1} \mathrm{H}$ NMR Spectrum of $(E)-3 \mathrm{~d}, 400 \mathrm{MHz}, \mathrm{CDCl}_{3}$

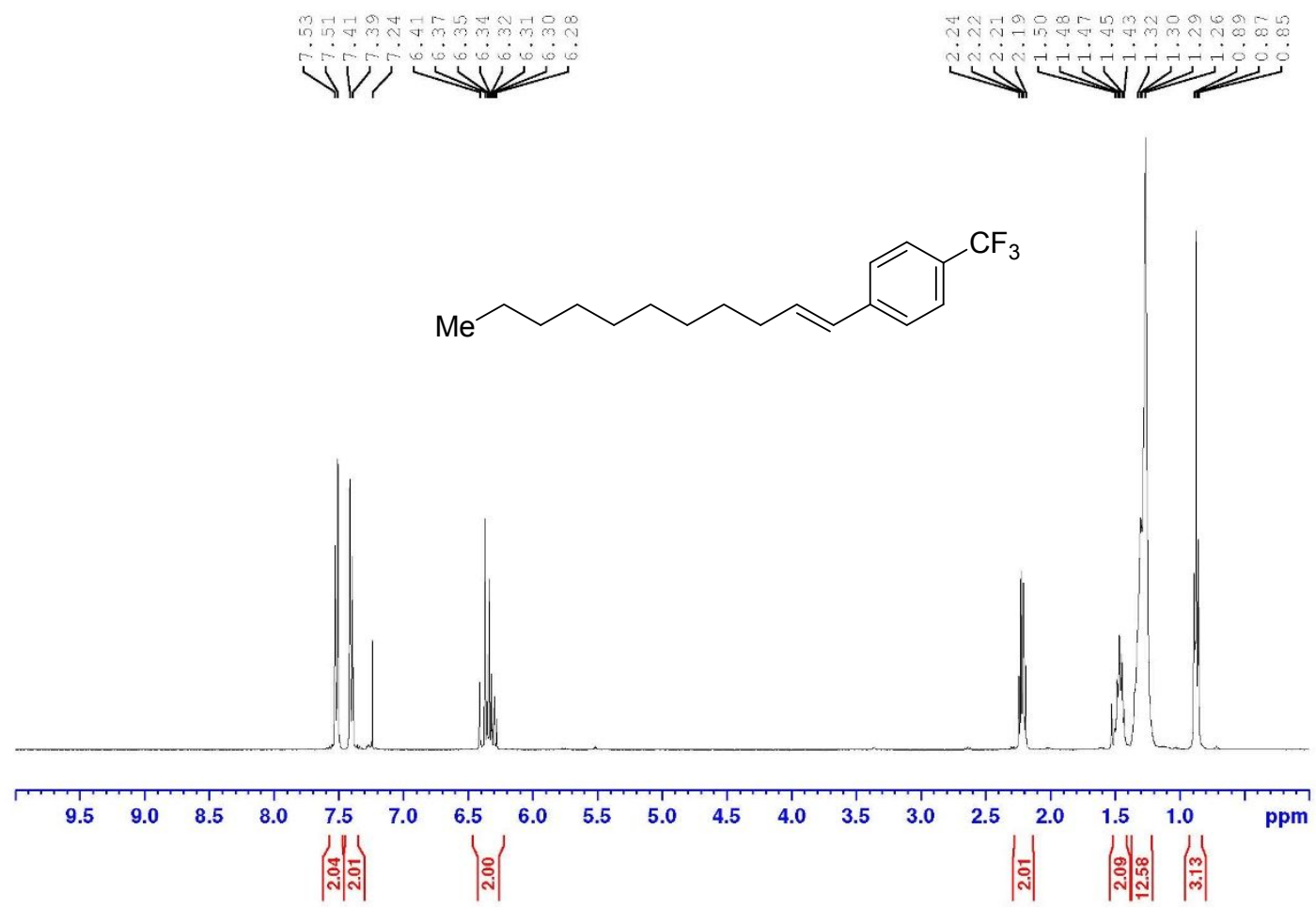

${ }^{13} \mathrm{C}$ NMR Spectrum of (E)-3d, $100 \mathrm{MHz}, \mathrm{CDCl}_{3}$ 


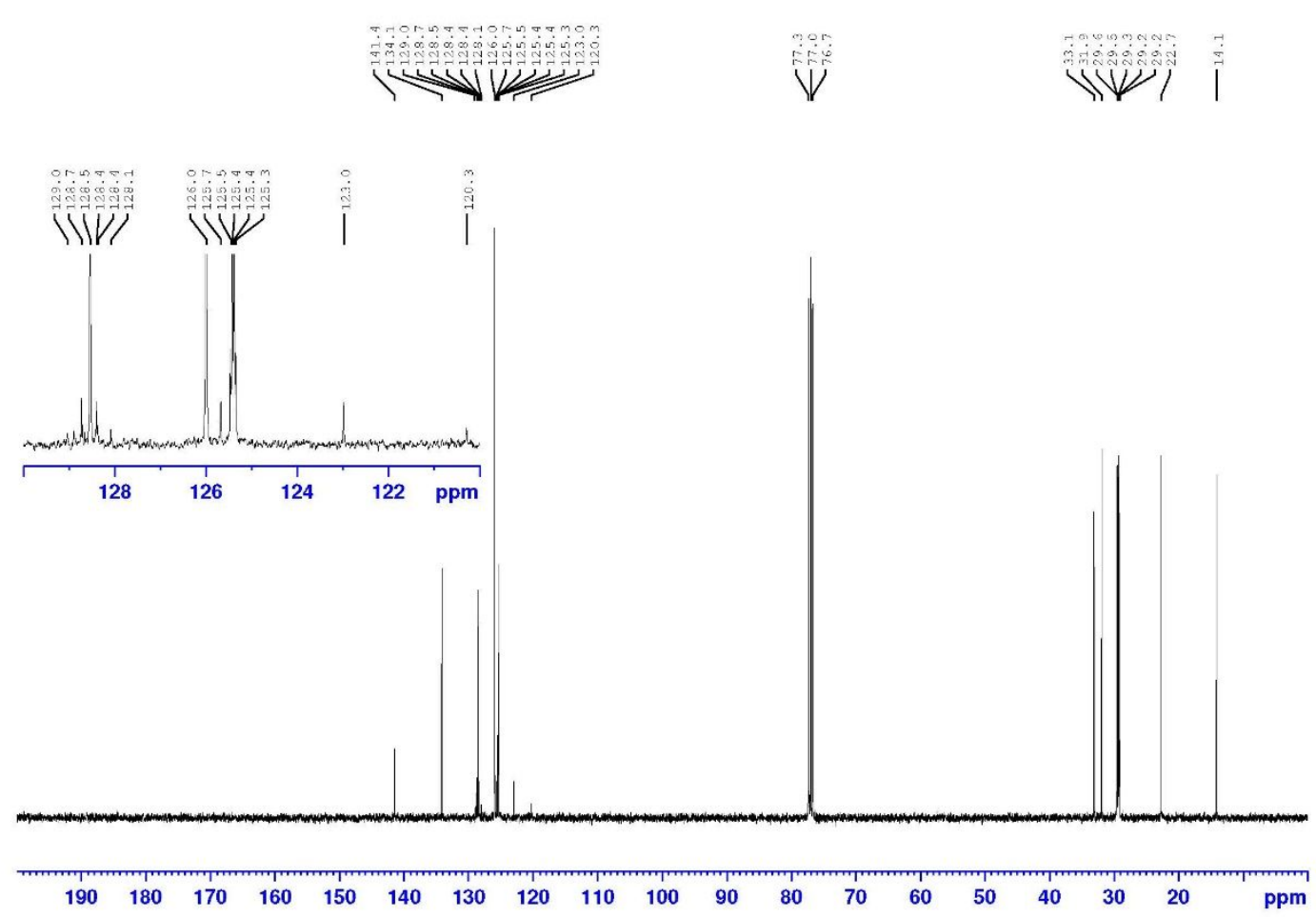

${ }^{1} \mathrm{H}$ NMR Spectrum of $(E)$ - and (Z)-3d, $400 \mathrm{MHz}, \mathrm{CDCl}_{3}$

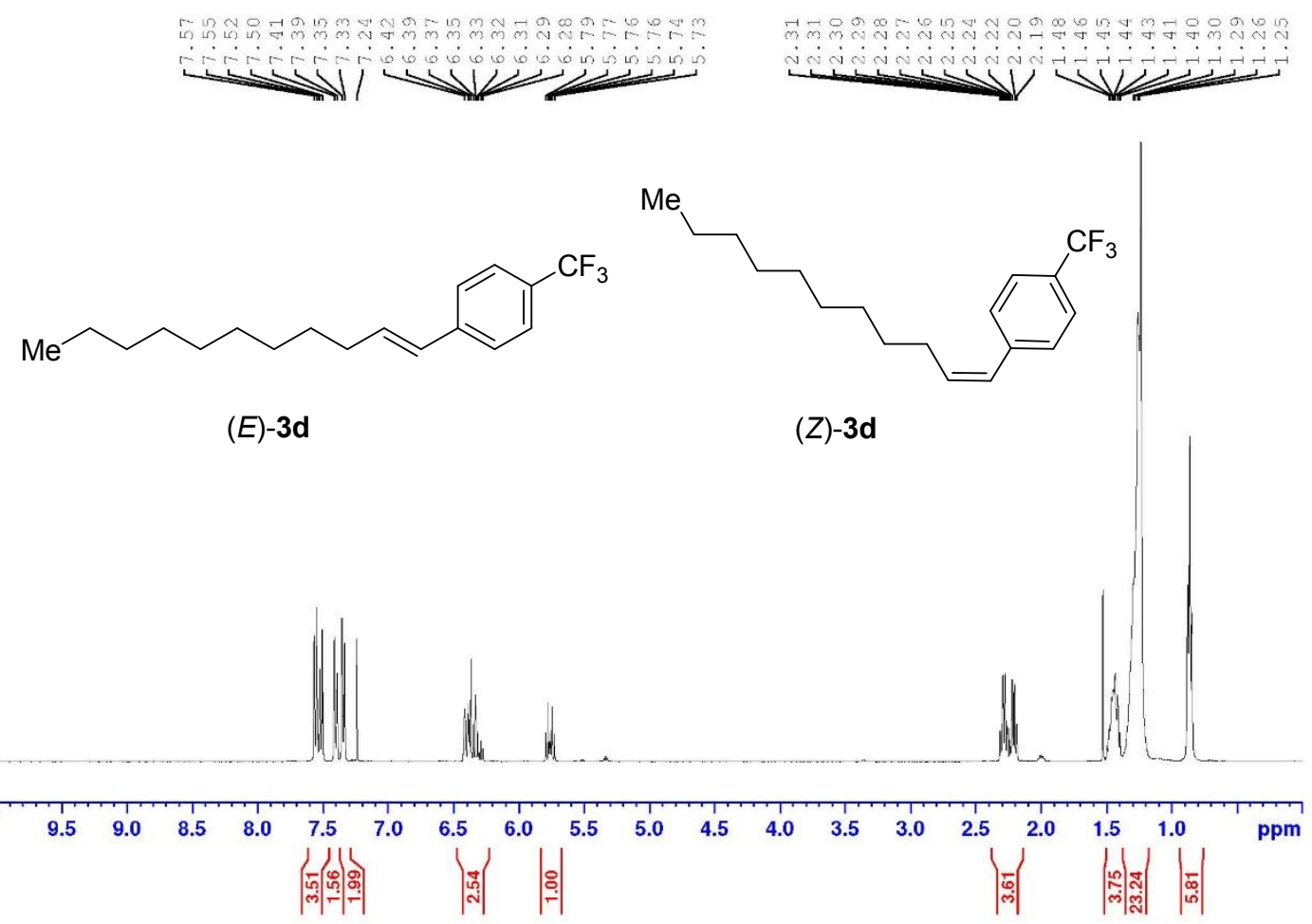

${ }^{13} \mathrm{C}$ NMR Spectrum of $(E)$ - and (Z)-3d, $100 \mathrm{MHz}, \mathrm{CDCl}_{3}$ 


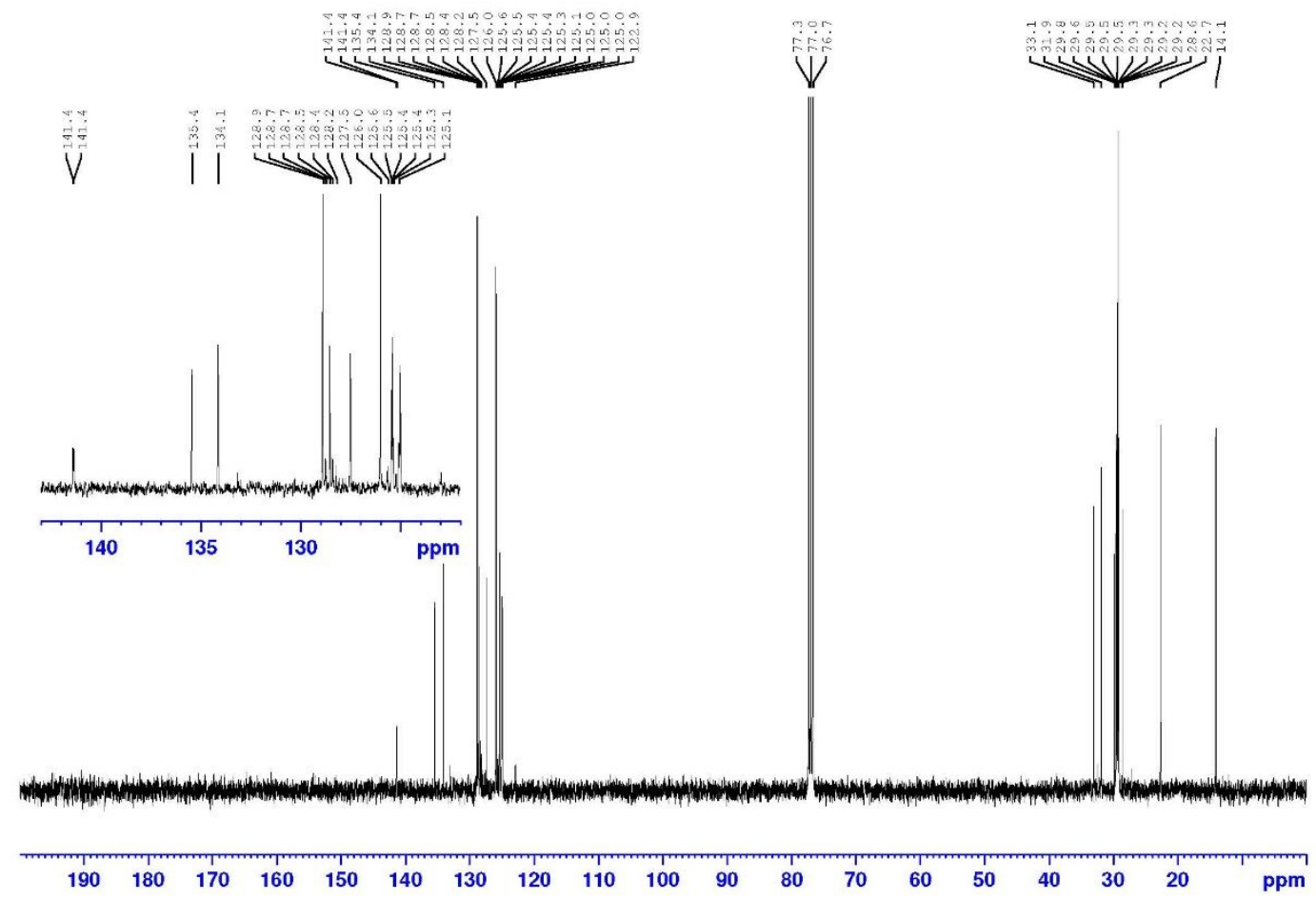

${ }^{1} \mathrm{H}$ NMR Spectrum of $(E)-3 e, 400 \mathrm{MHz}, \mathrm{CDCl}_{3}$

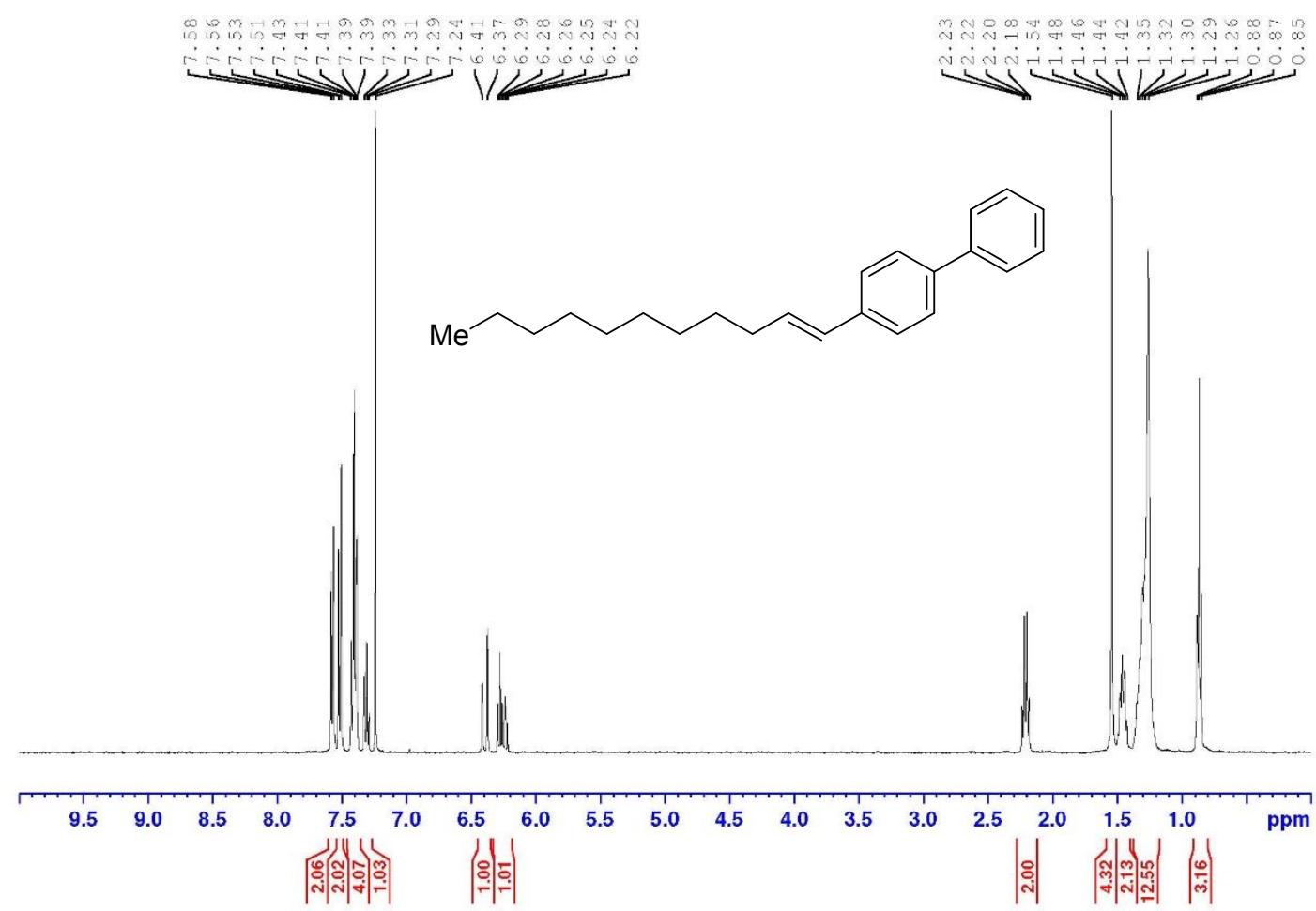

${ }^{13} \mathrm{C}$ NMR Spectrum of $(E)-3 e, 100 \mathrm{MHz}, \mathrm{CDCl}_{3}$ 

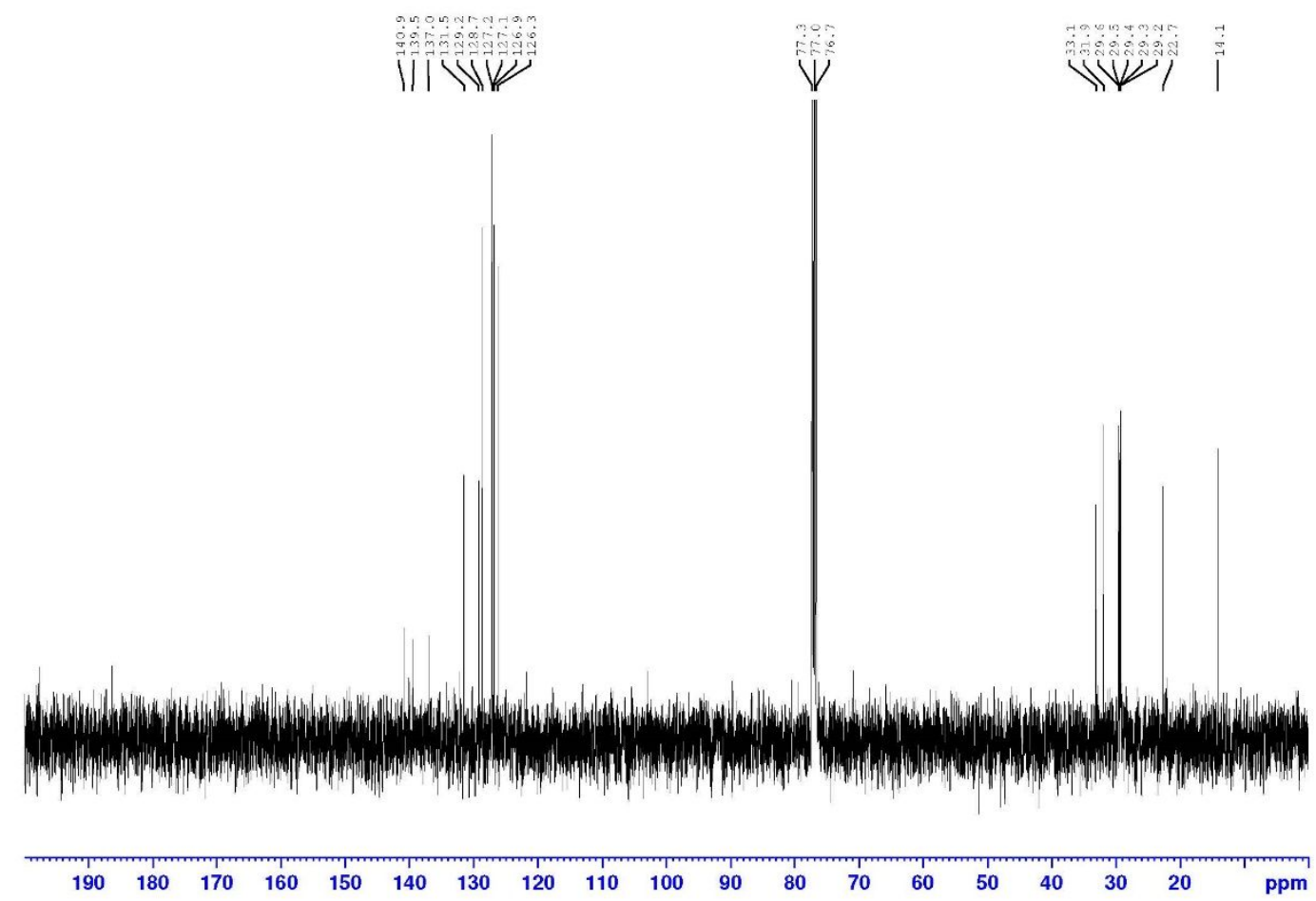

${ }^{1} \mathrm{H}$ NMR Spectrum of $(E)-3 f, 400 \mathrm{MHz}, \mathrm{CDCl}_{3}$

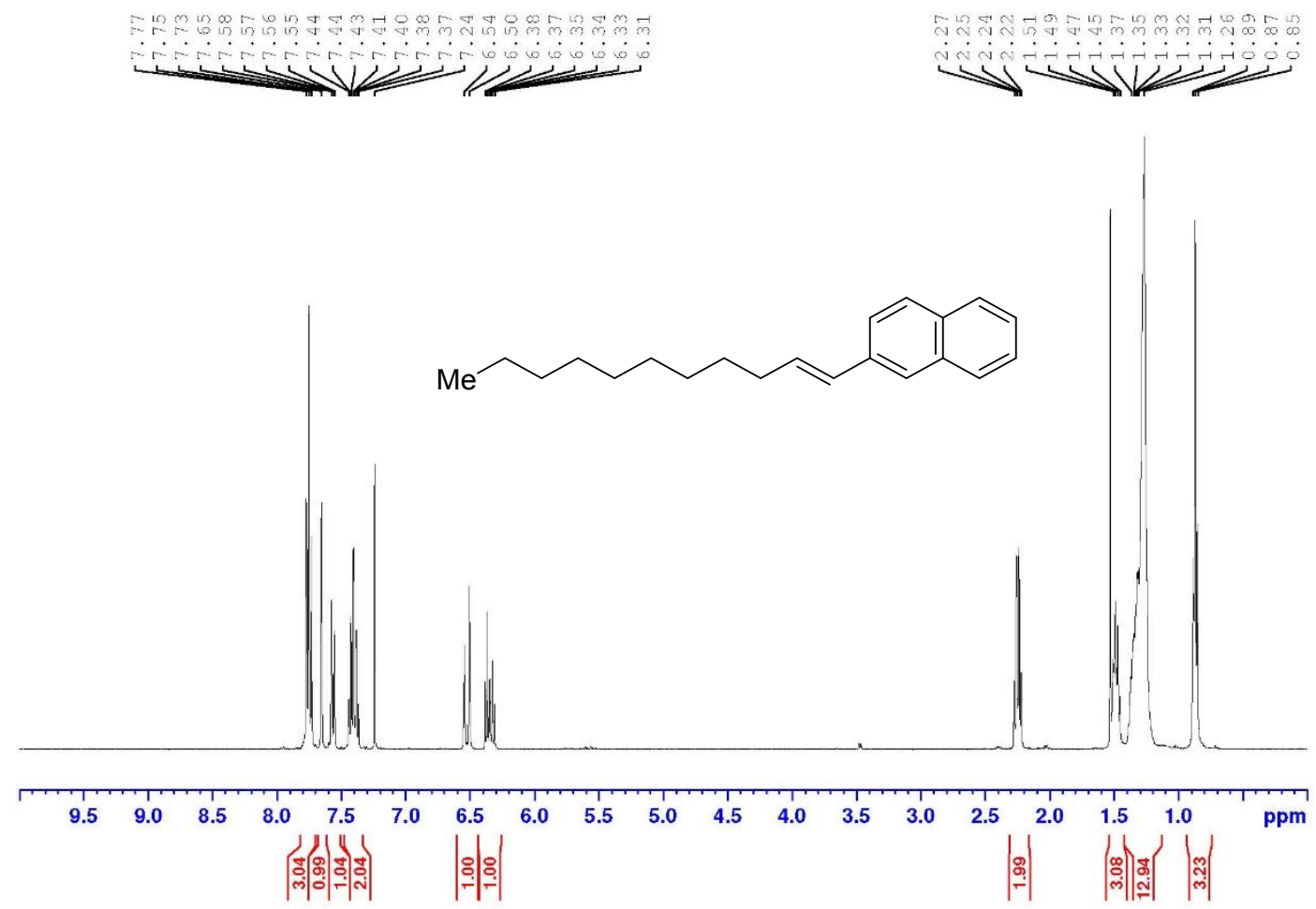

${ }^{13} \mathrm{C}$ NMR Spectrum of (E)-3f, $100 \mathrm{MHz}, \mathrm{CDCl}_{3}$ 

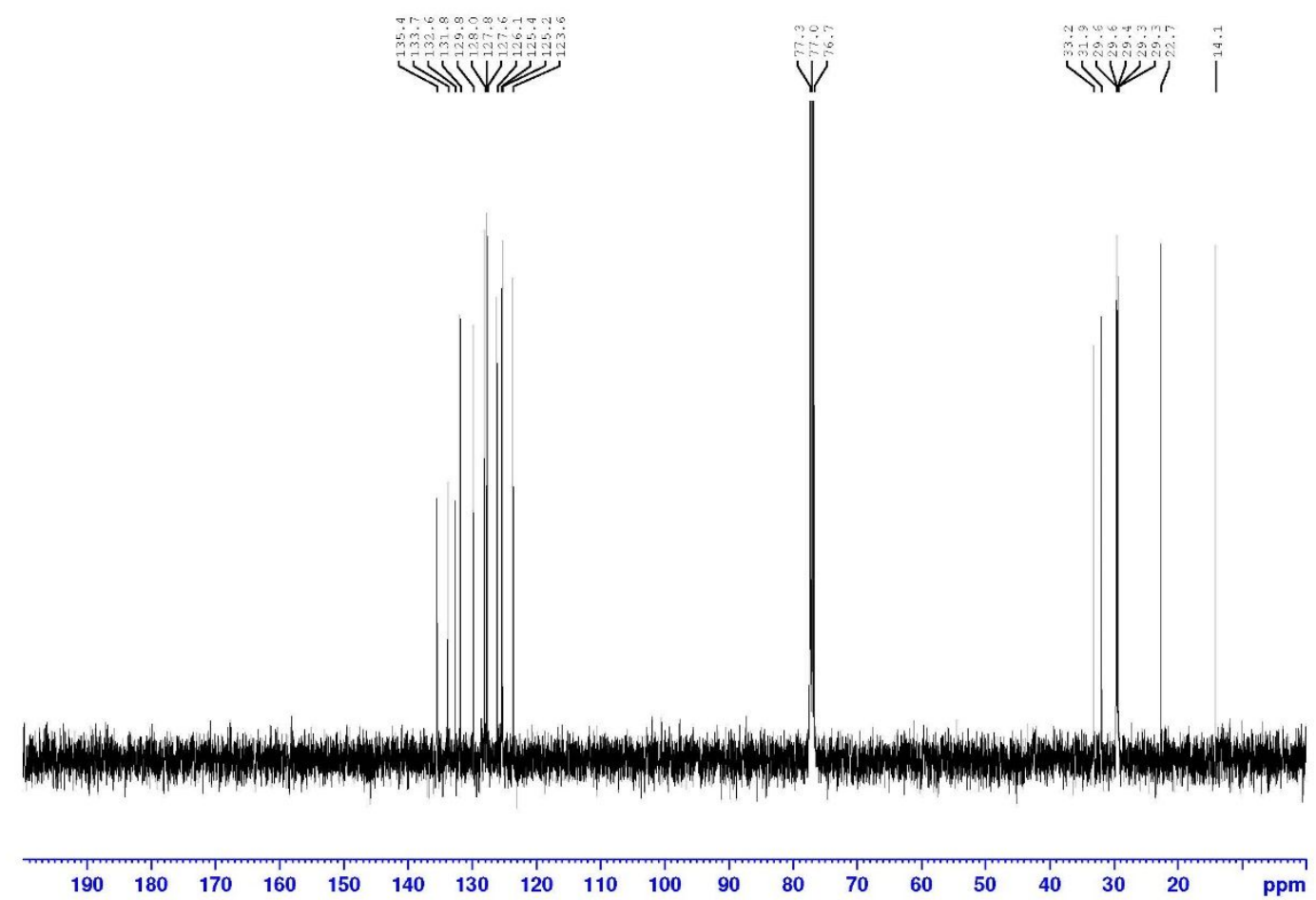

${ }^{1} \mathrm{H}$ NMR Spectrum of $(E)-3 g, 400 \mathrm{MHz}, \mathrm{CDCl}_{3}$

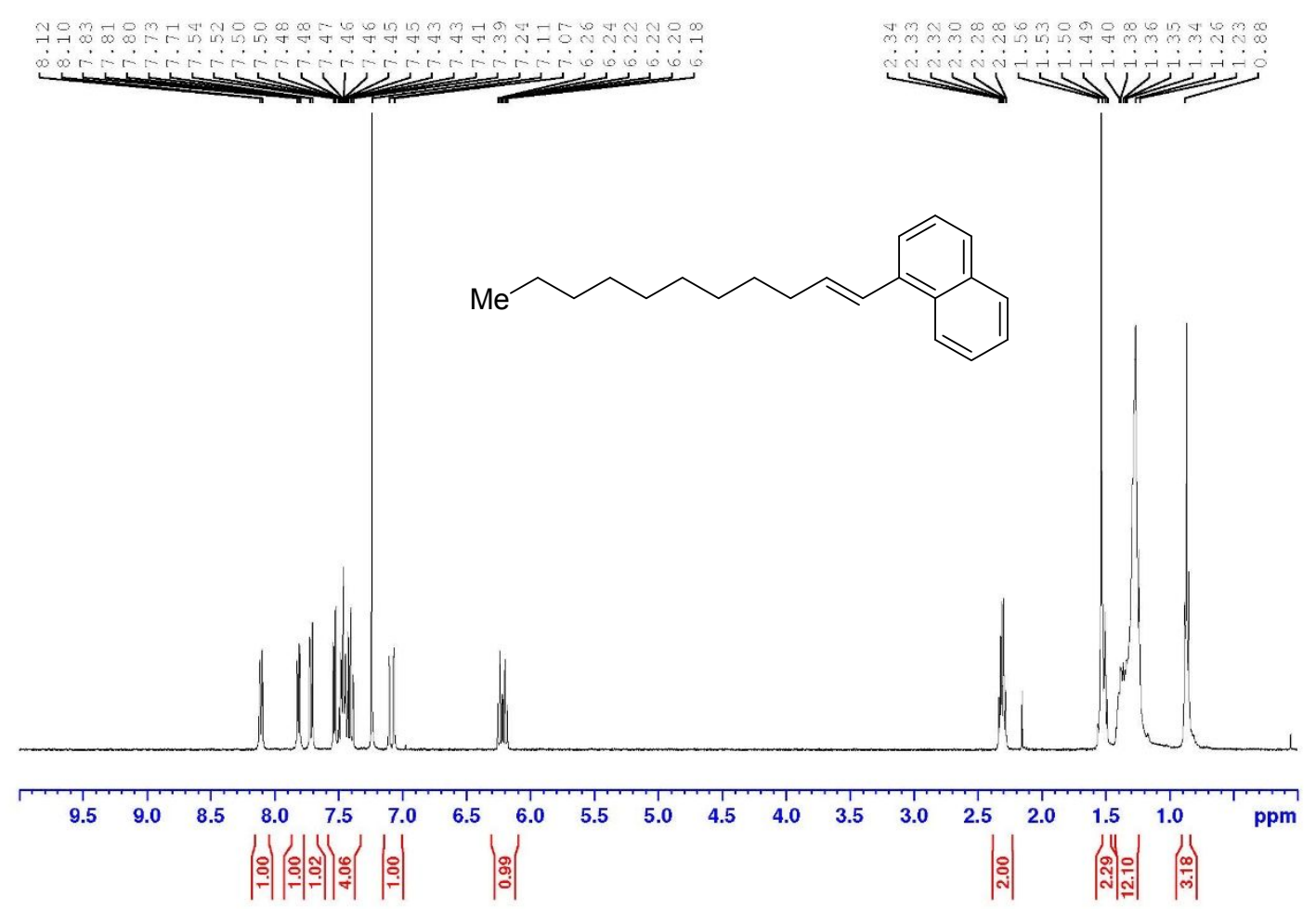

${ }^{13} \mathrm{C}$ NMR Spectrum of (E)-3g, $100 \mathrm{MHz}, \mathrm{CDCl}_{3}$ 


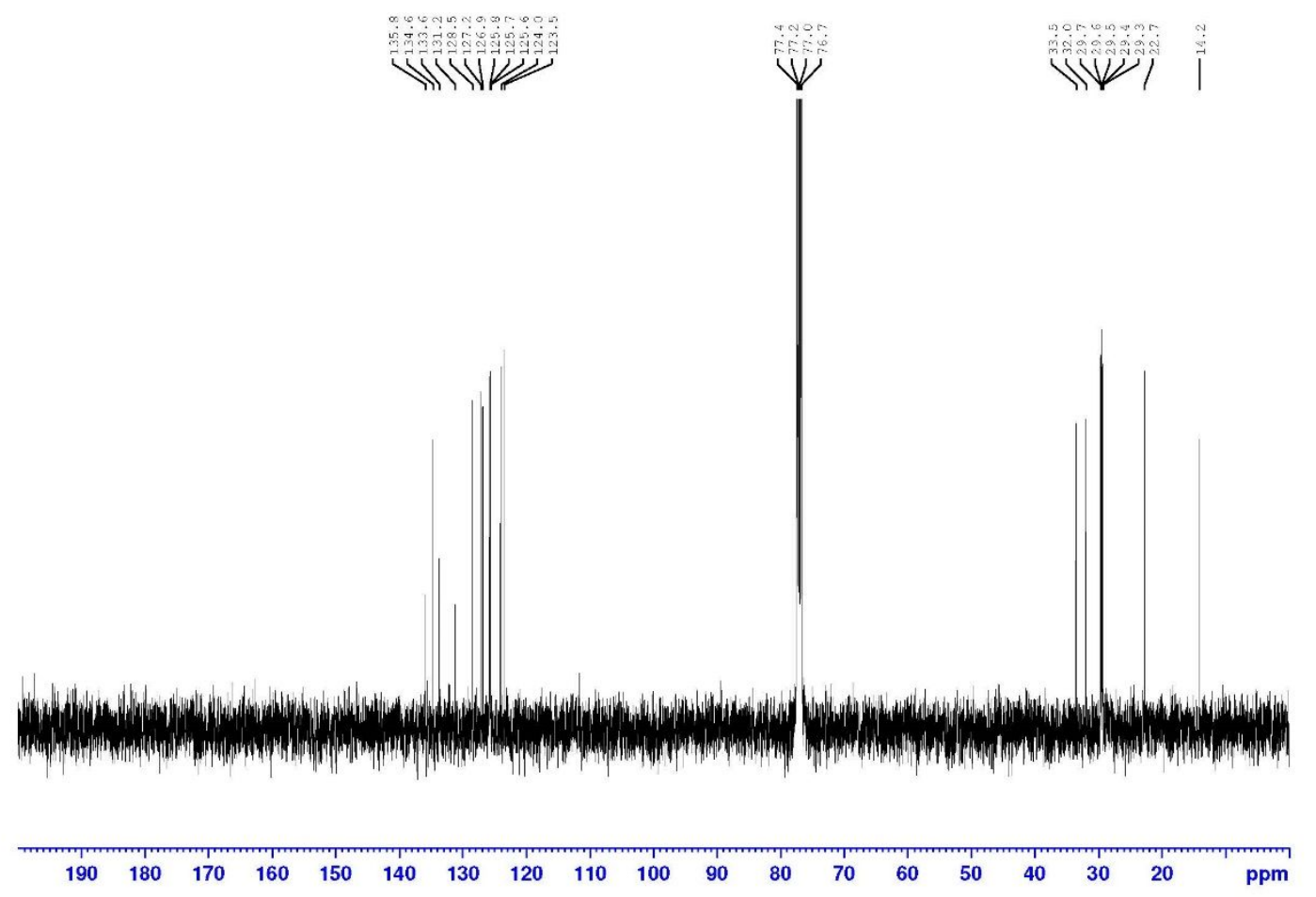

${ }^{1} \mathrm{H}$ NMR Spectrum of (Z)-3g, $400 \mathrm{MHz}, \mathrm{CDCl}_{3}$

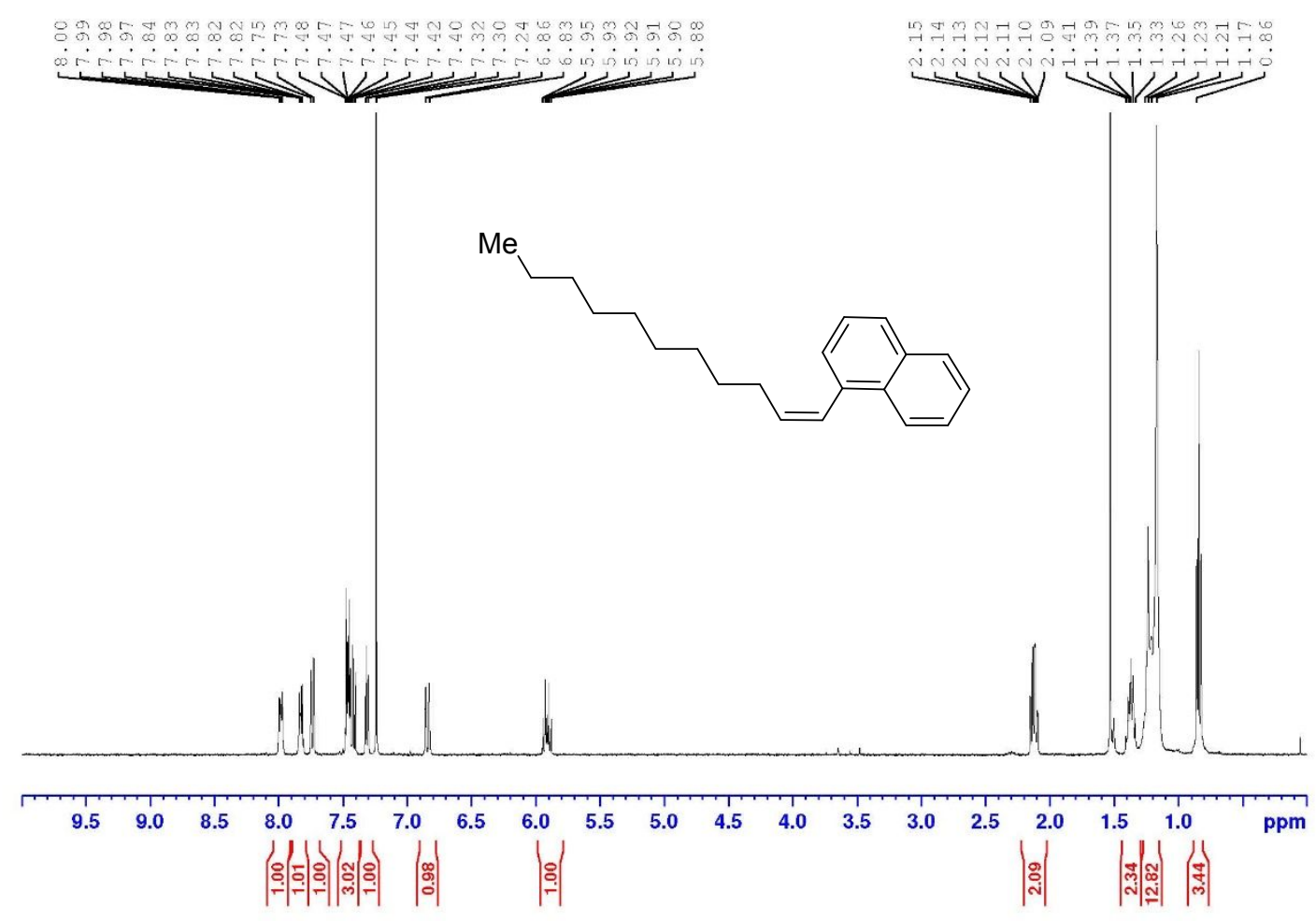

${ }^{13} \mathrm{C}$ NMR Spectrum of (Z)-3g, $100 \mathrm{MHz}, \mathrm{CDCl}_{3}$ 

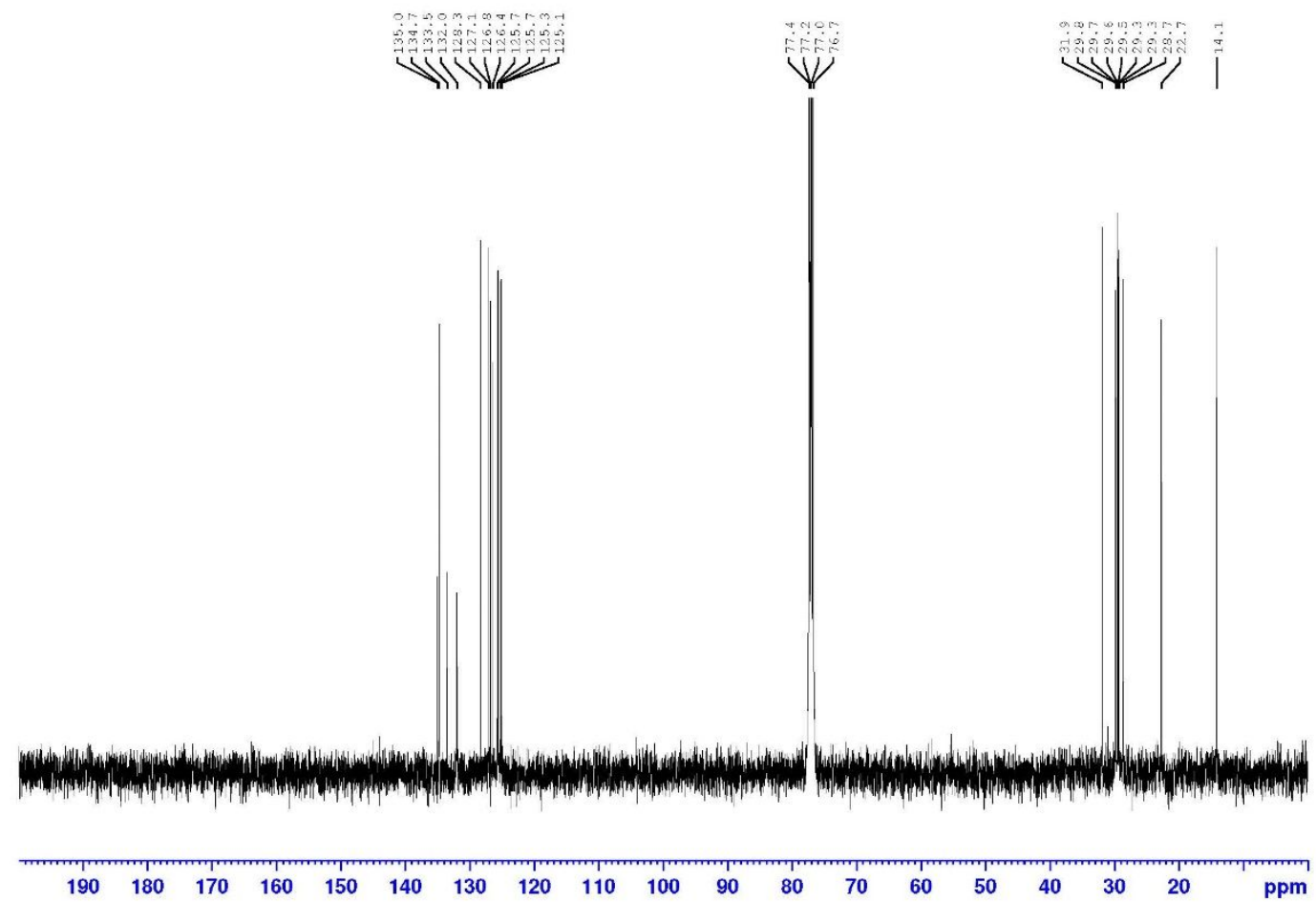

${ }^{1} \mathrm{H}$ NMR Spectrum of $(E)-3 \mathrm{~h}, 400 \mathrm{MHz}, \mathrm{CDCl}_{3}$

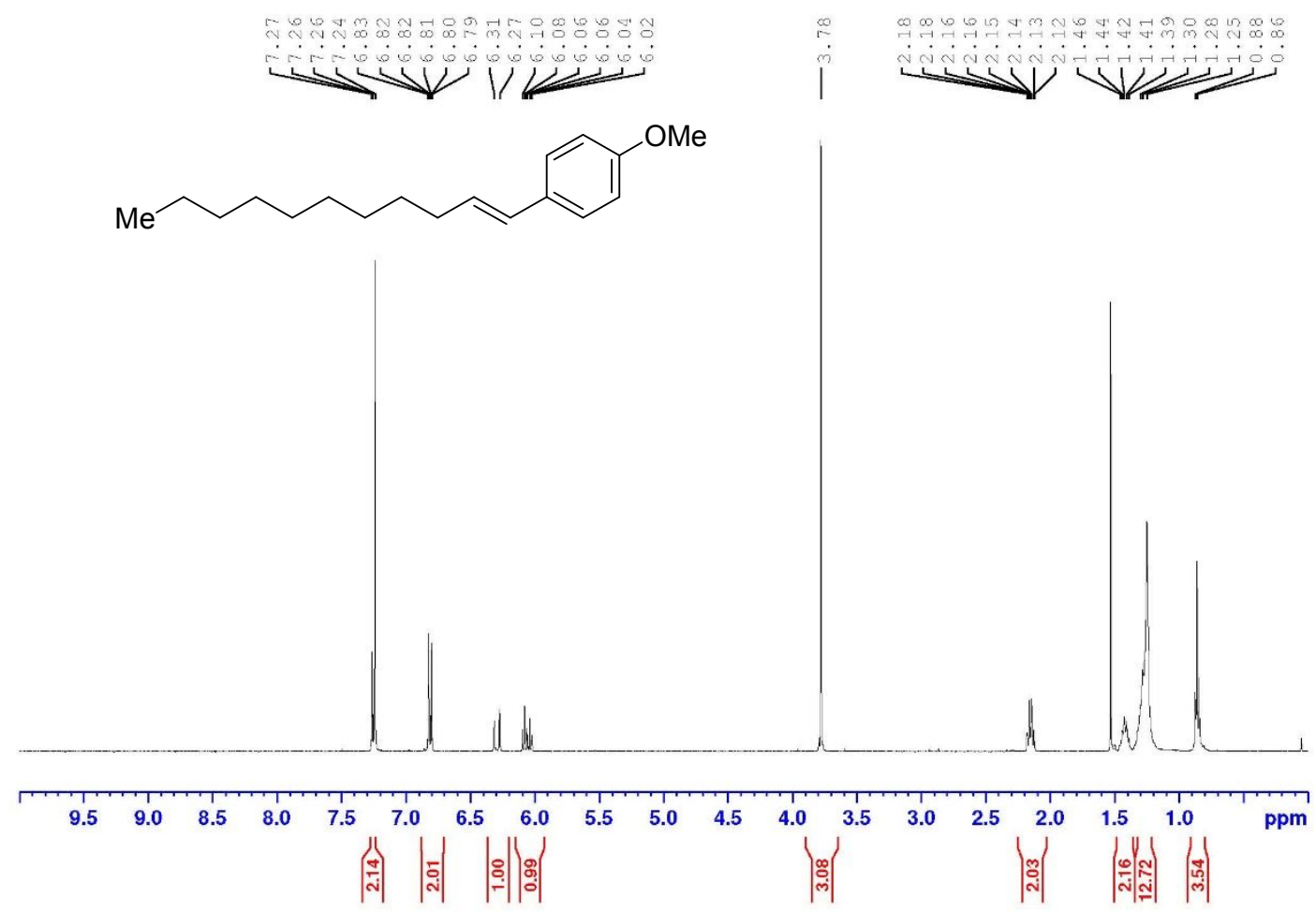

${ }^{13} \mathrm{C}$ NMR Spectrum of (E)-3h, $100 \mathrm{MHz}, \mathrm{CDCl}_{3}$ 


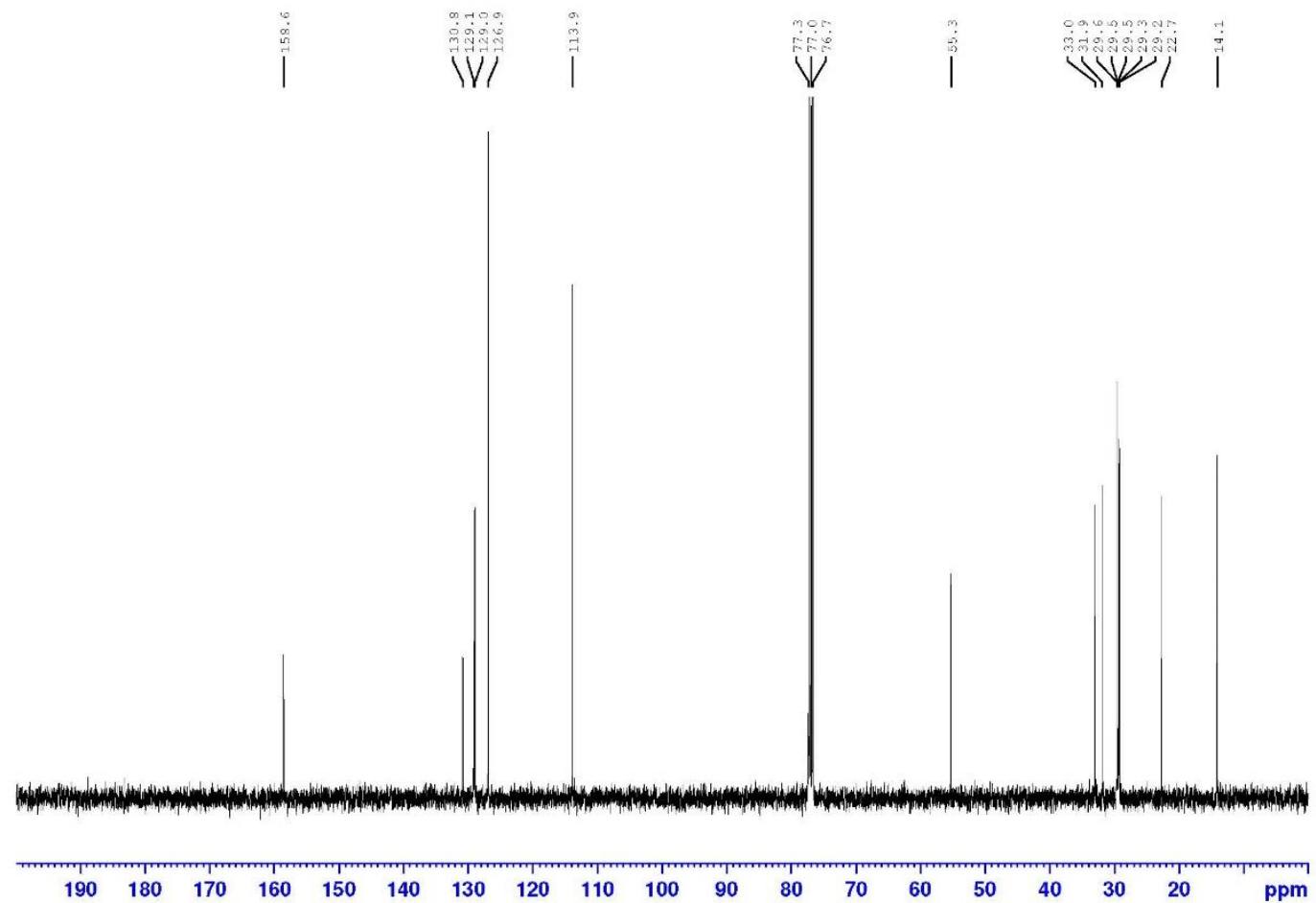

${ }^{1} \mathrm{H}$ NMR Spectrum of (Z)-3h, $400 \mathrm{MHz}, \mathrm{CDCl}_{3}$

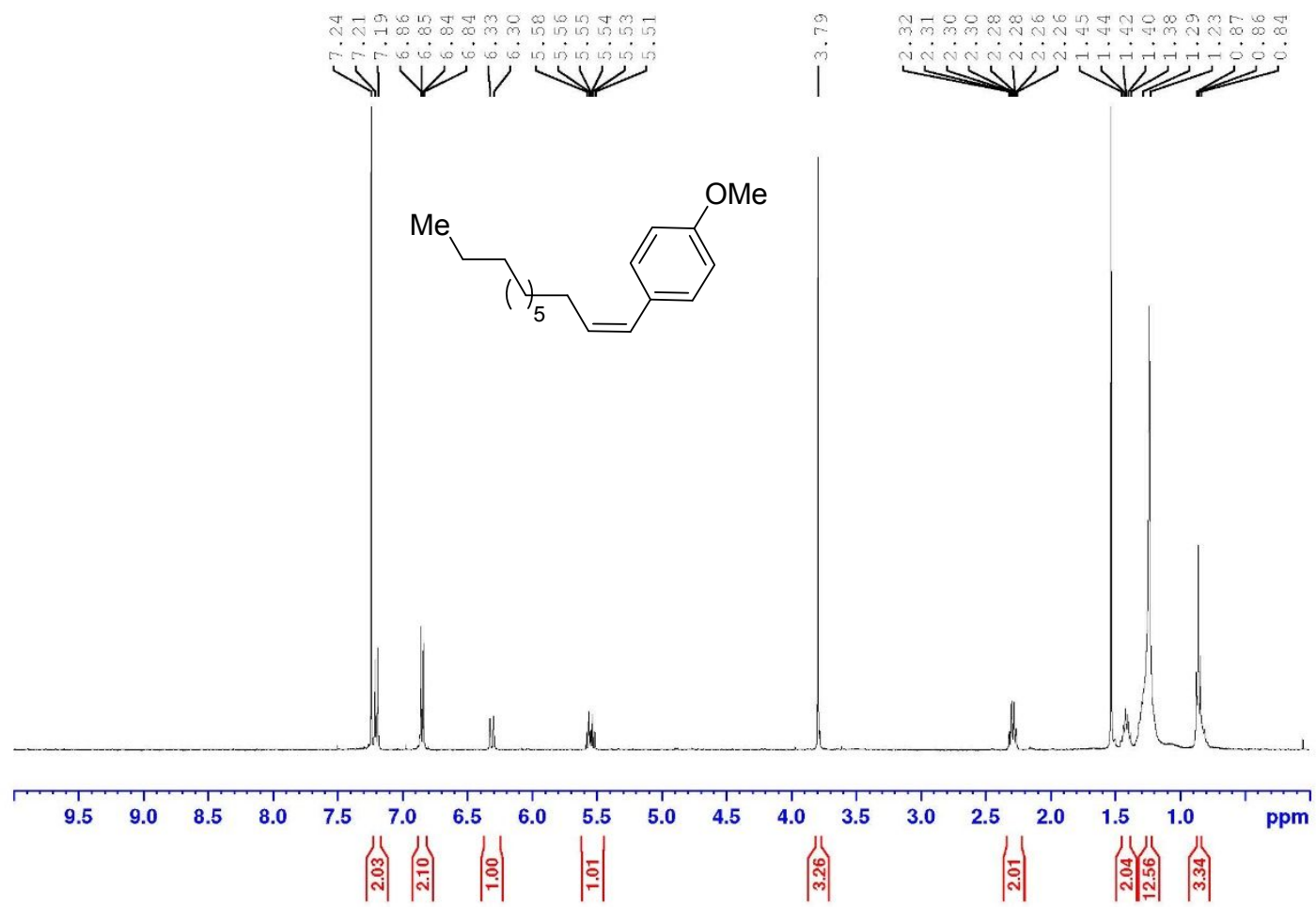

${ }^{13} \mathrm{C}$ NMR Spectrum of (Z)-3h, $100 \mathrm{MHz}, \mathrm{CDCl}_{3}$ 

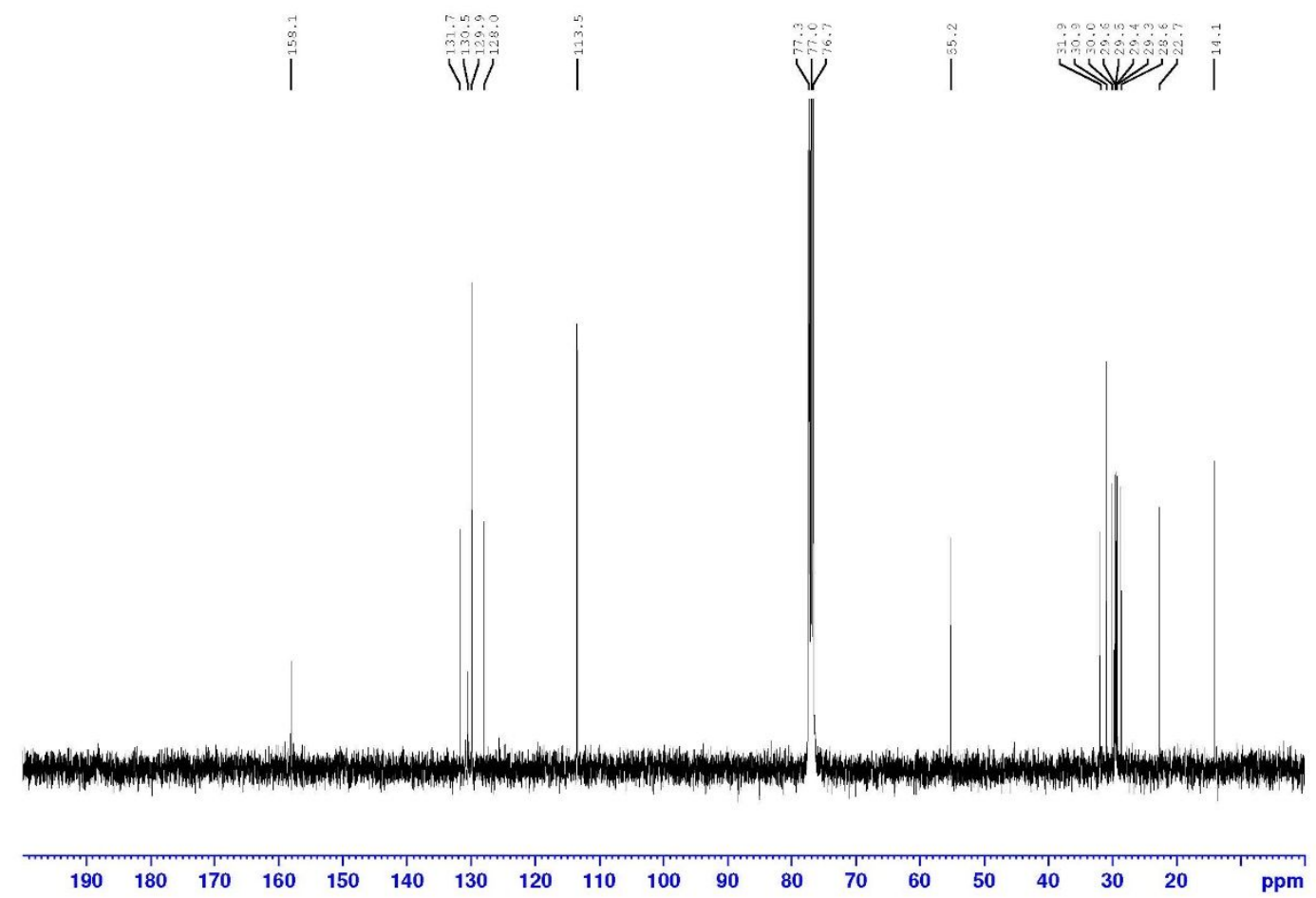

${ }^{1} \mathrm{H}$ NMR Spectrum of $(E)-3 \mathbf{i}, 400 \mathrm{MHz}, \mathrm{CDCl}_{3}$

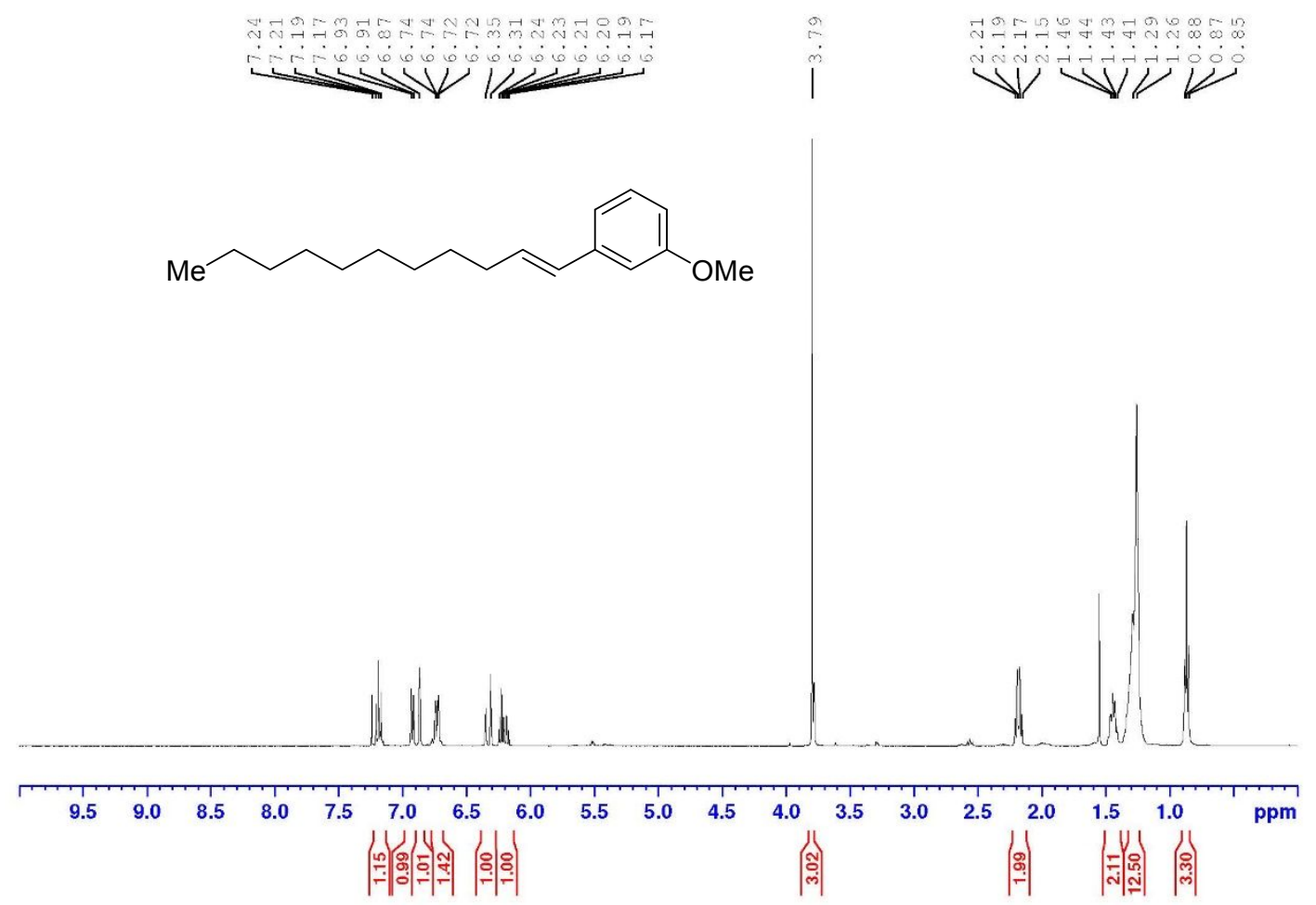

${ }^{13} \mathrm{C}$ NMR Spectrum of (E)-3i, $100 \mathrm{MHz}, \mathrm{CDCl}_{3}$ 


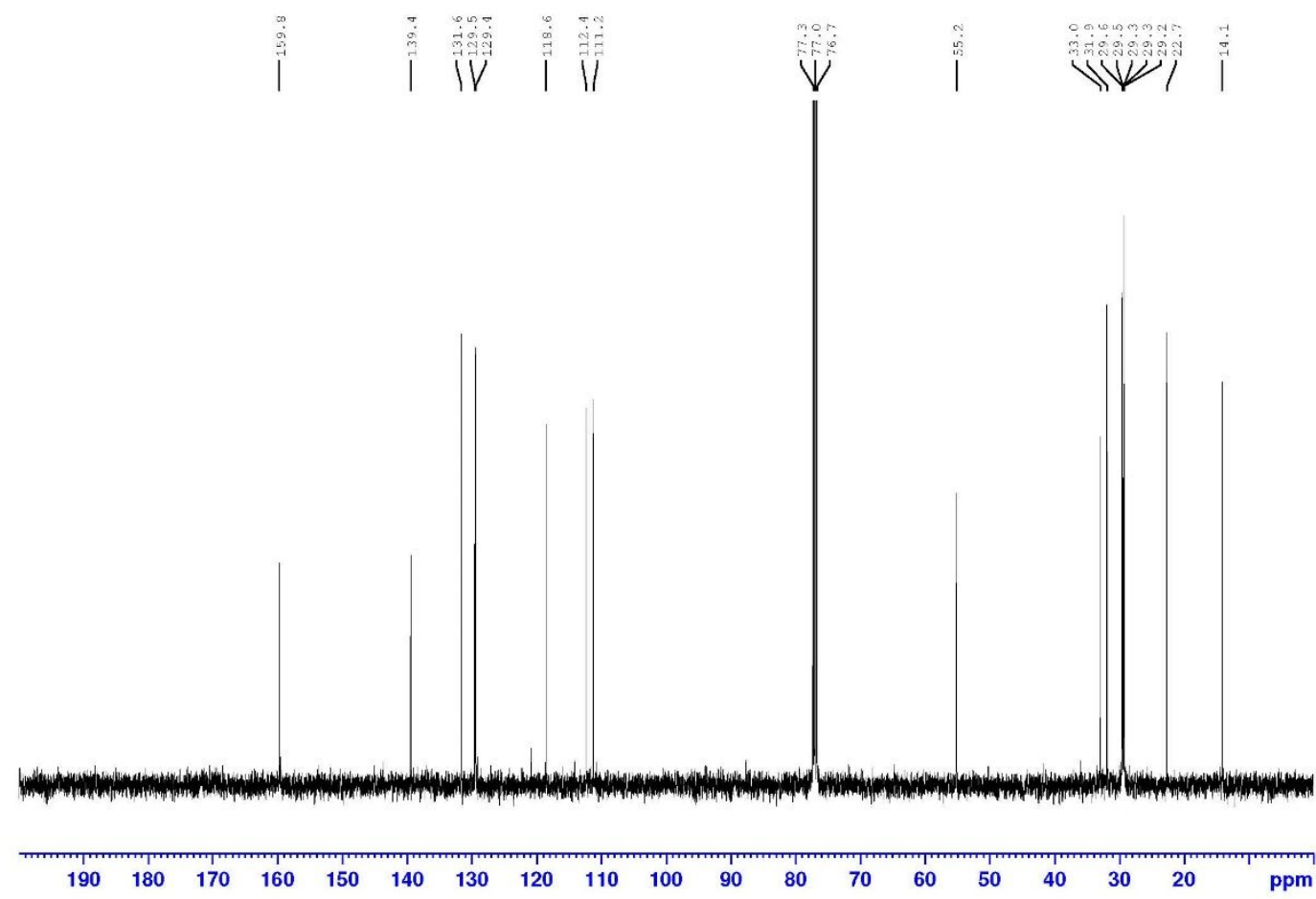

${ }^{1} \mathrm{H}$ NMR Spectrum of $(E)-3 \mathbf{j}, 400 \mathrm{MHz}, \mathrm{CDCl}_{3}$

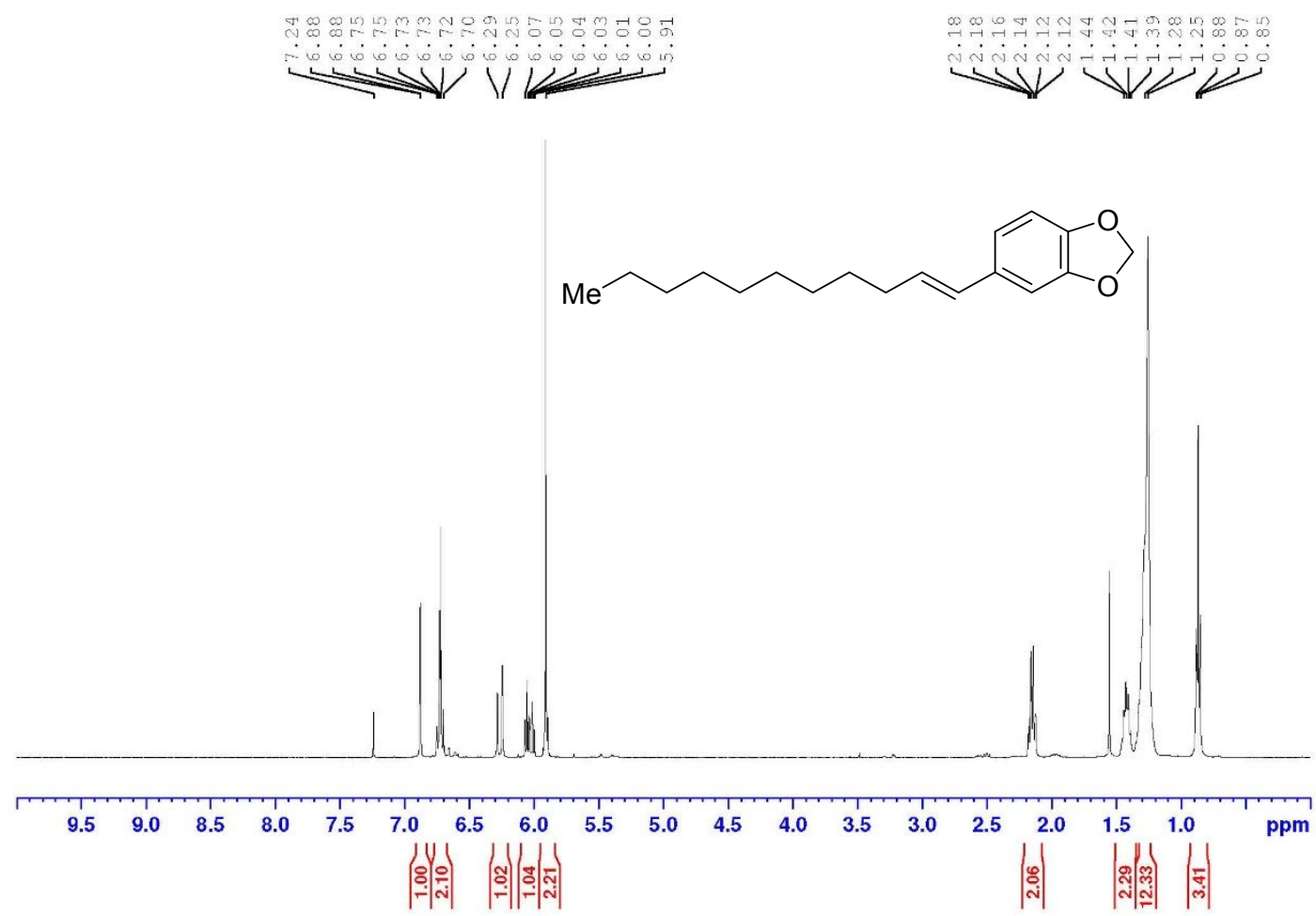

${ }^{13} \mathrm{C}$ NMR Spectrum of $(E)-3 \mathbf{j}, 100 \mathrm{MHz}, \mathrm{CDCl}_{3}$ 


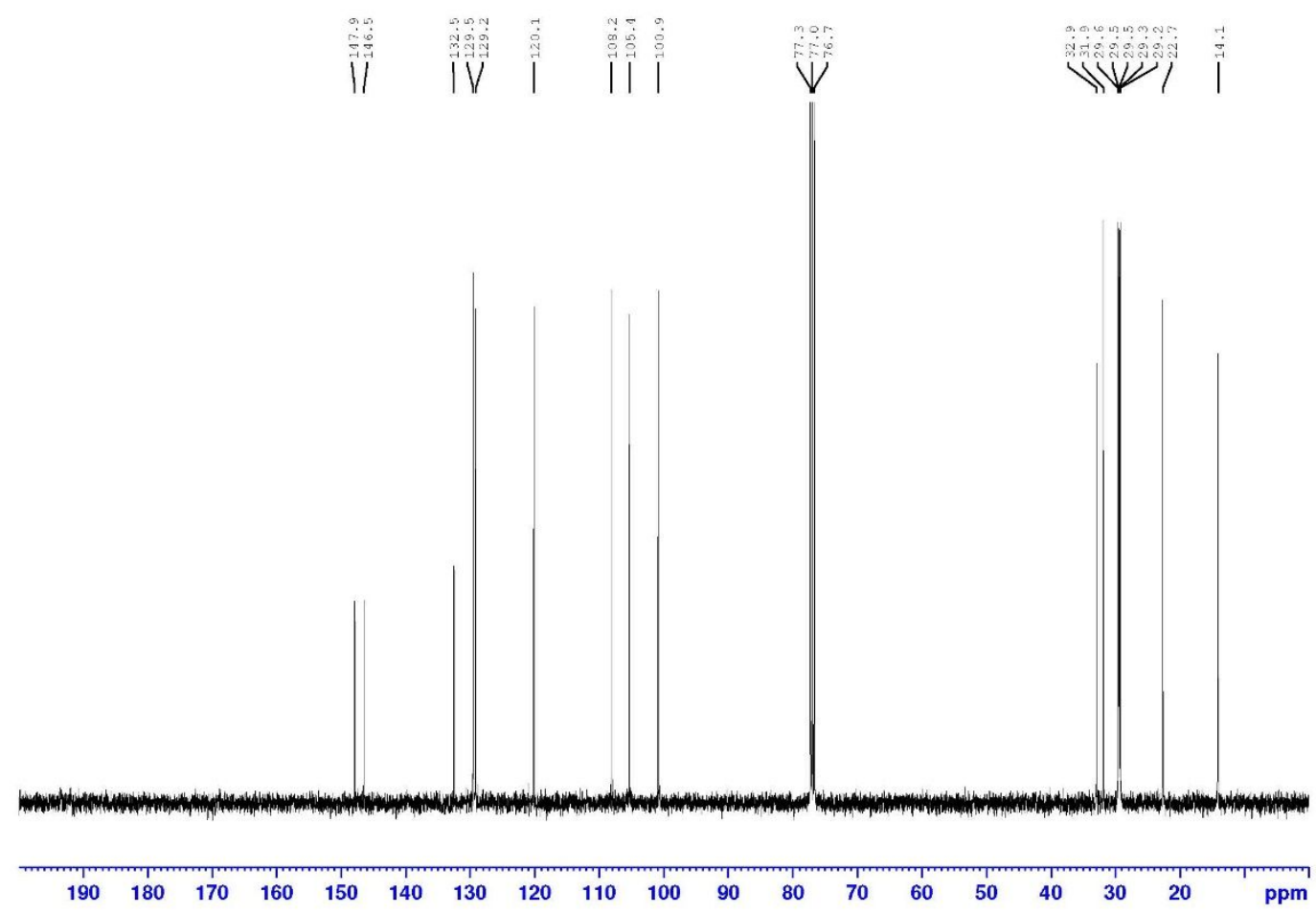

${ }^{1} \mathrm{H}$ NMR Spectrum of $(E)-3 \mathbf{k}, 400 \mathrm{MHz}, \mathrm{CDCl}_{3}$

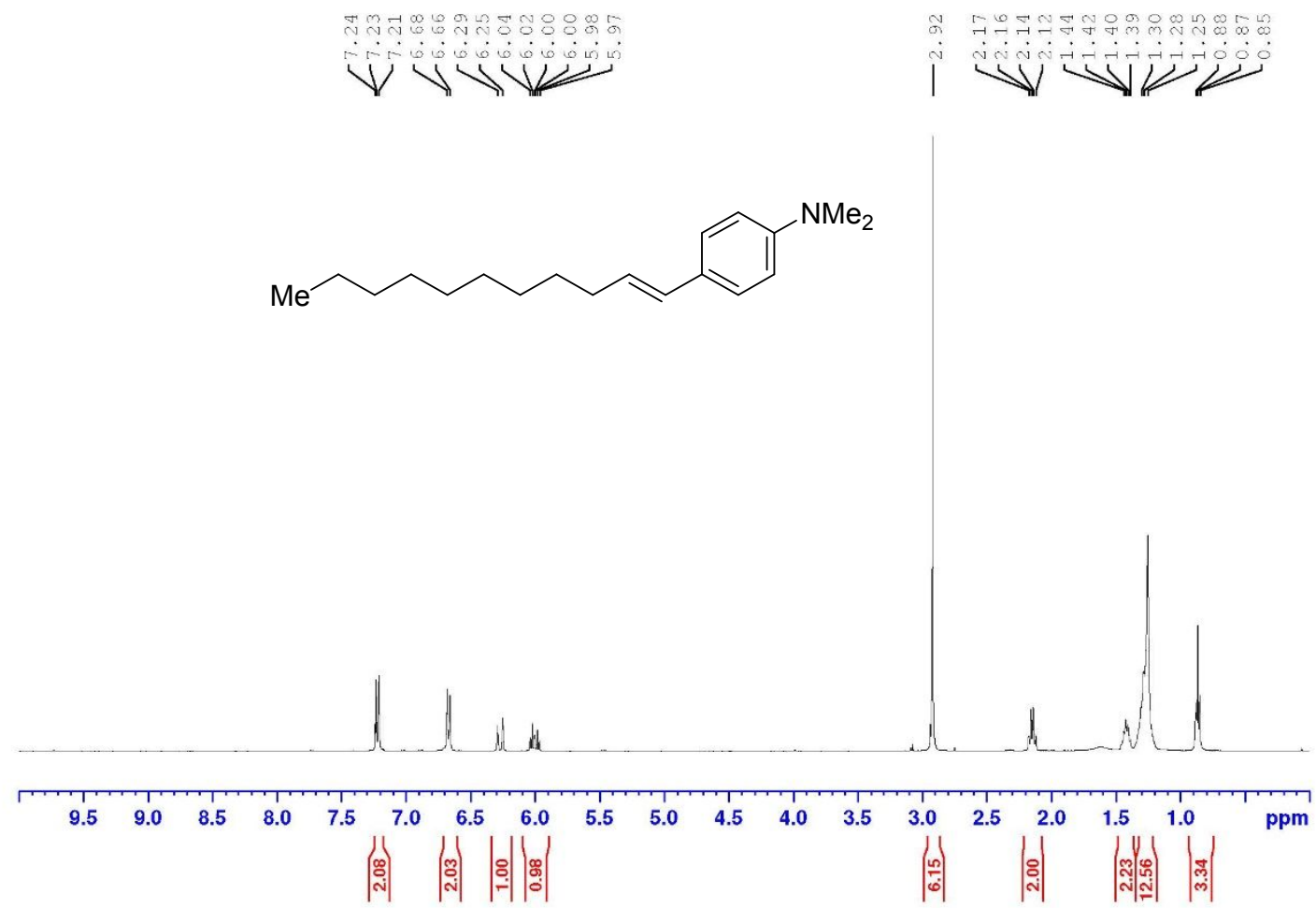

${ }^{13} \mathrm{C}$ NMR Spectrum of (E)-3k, $100 \mathrm{MHz}, \mathrm{CDCl}_{3}$ 


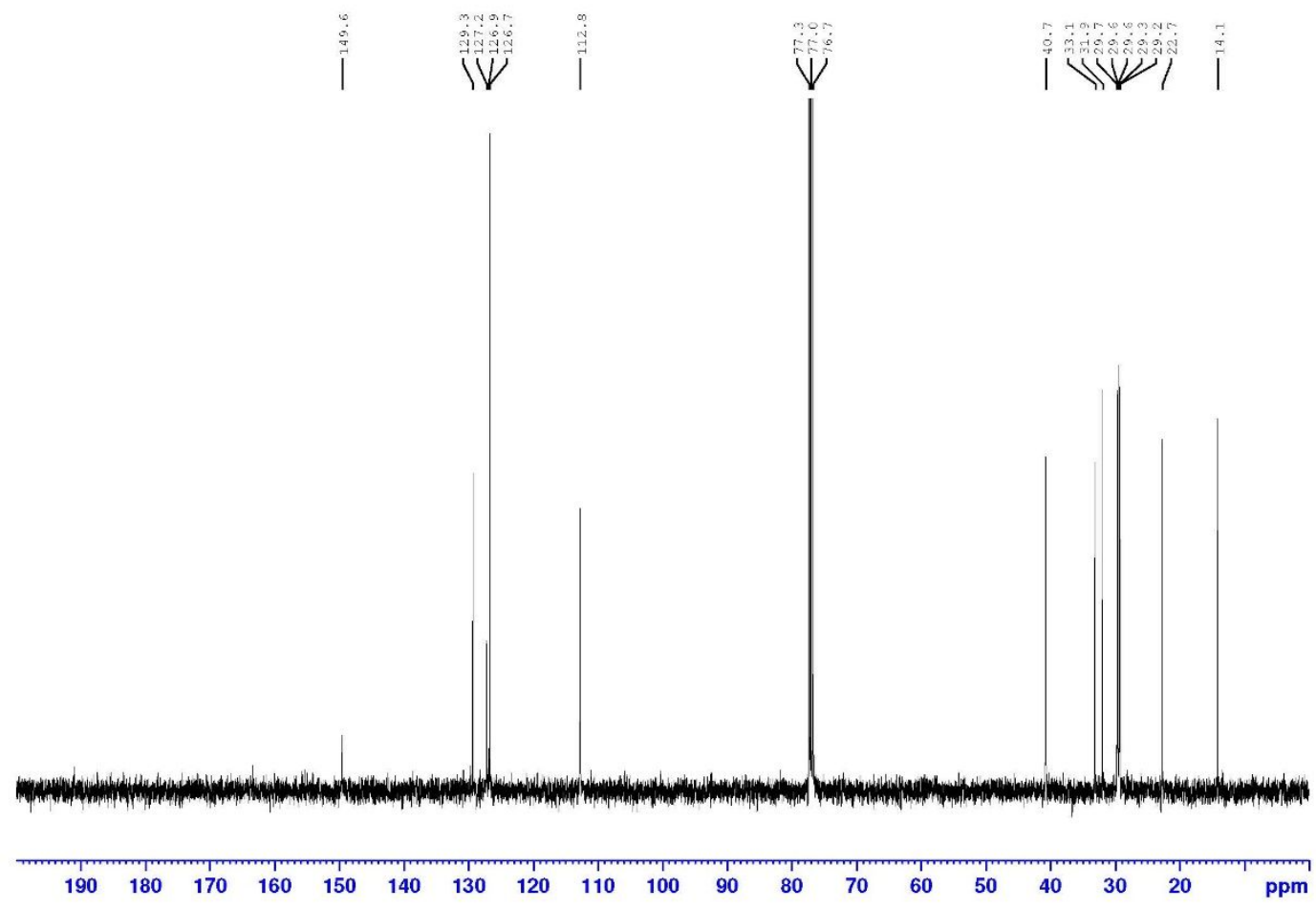

${ }^{1} \mathrm{H}$ NMR Spectrum of $(E)-3 \mathrm{I}, 400 \mathrm{MHz}, \mathrm{CDCl}_{3}$

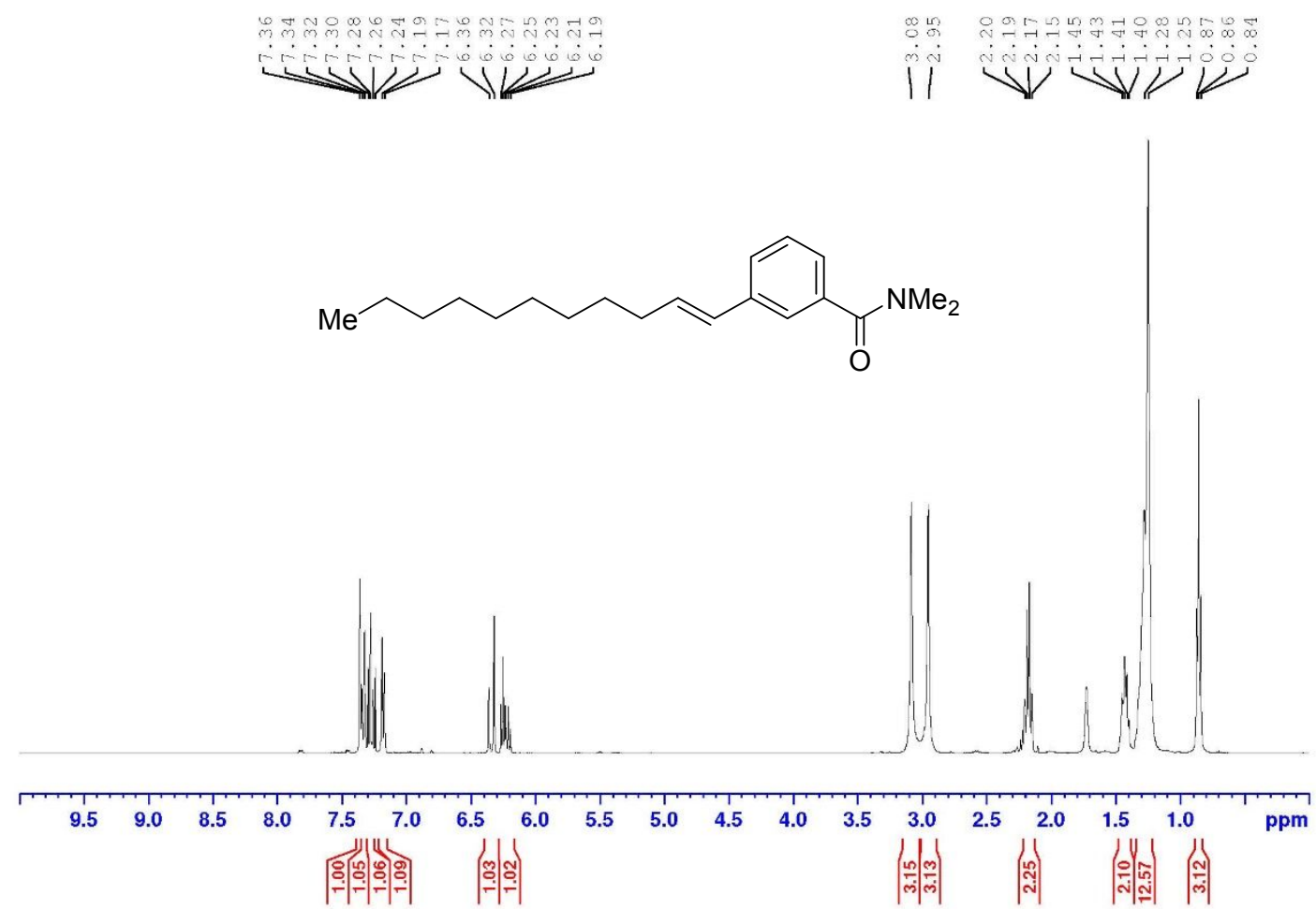

${ }^{13} \mathrm{C}$ NMR Spectrum of $(E)-3 \mathbf{l}, 100 \mathrm{MHz}, \mathrm{CDCl}_{3}$ 


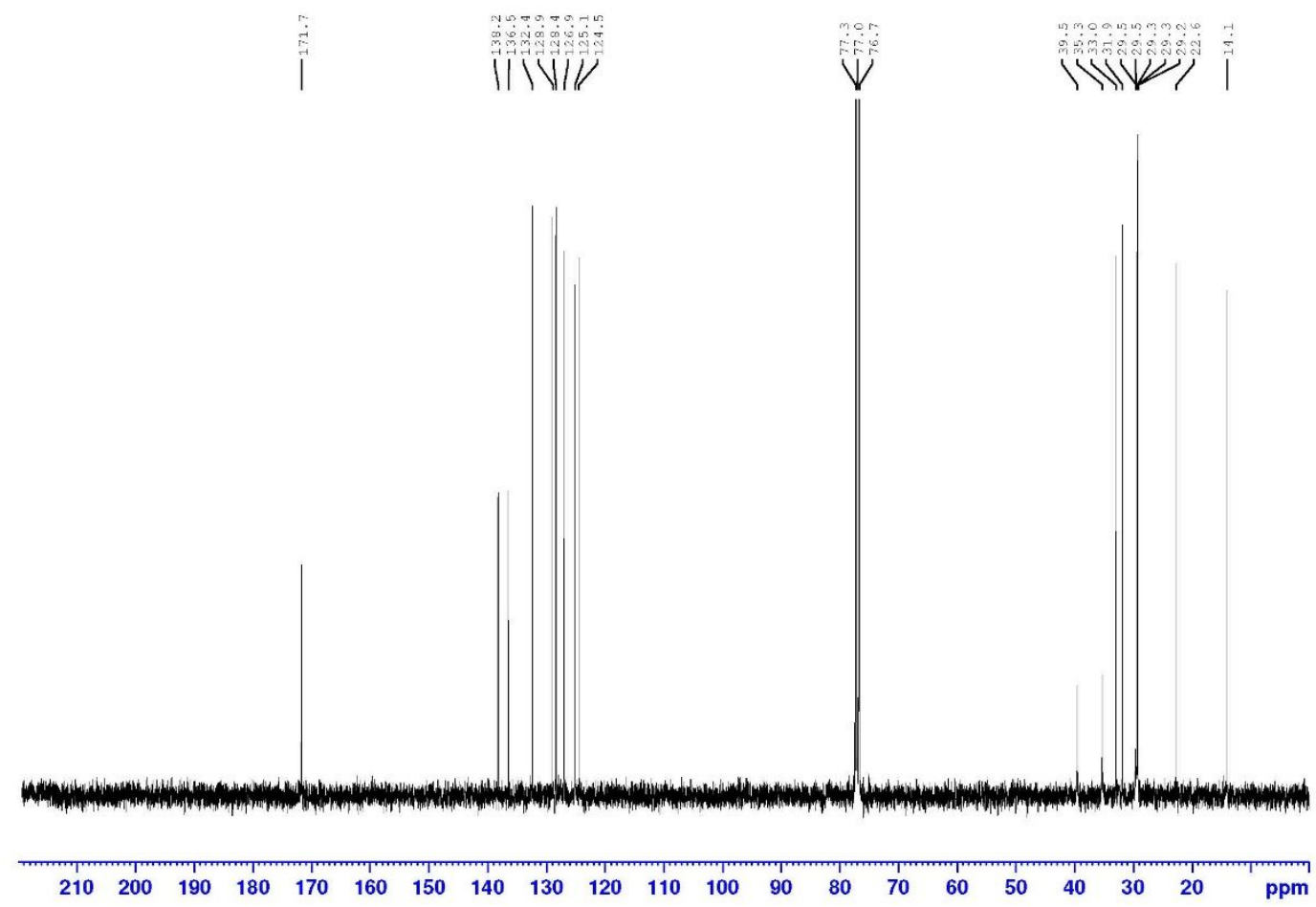

${ }^{1} \mathrm{H}$ NMR Spectrum of $(E)-3 \mathrm{~m}, 400 \mathrm{MHz}, \mathrm{CDCl}_{3}$

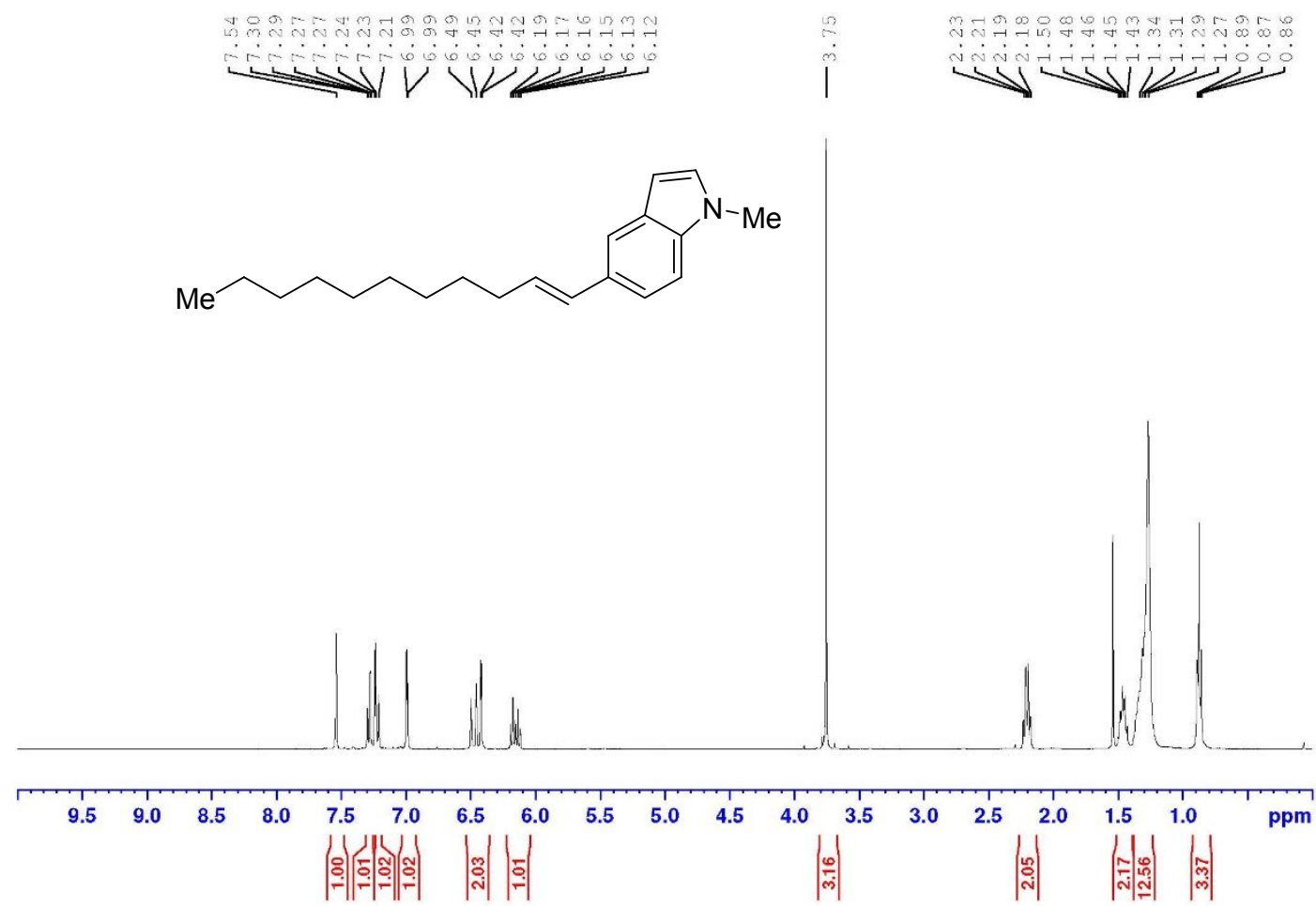

${ }^{13} \mathrm{C}$ NMR Spectrum of $(E)-3 \mathrm{~m}, 100 \mathrm{MHz}, \mathrm{CDCl}_{3}$ 


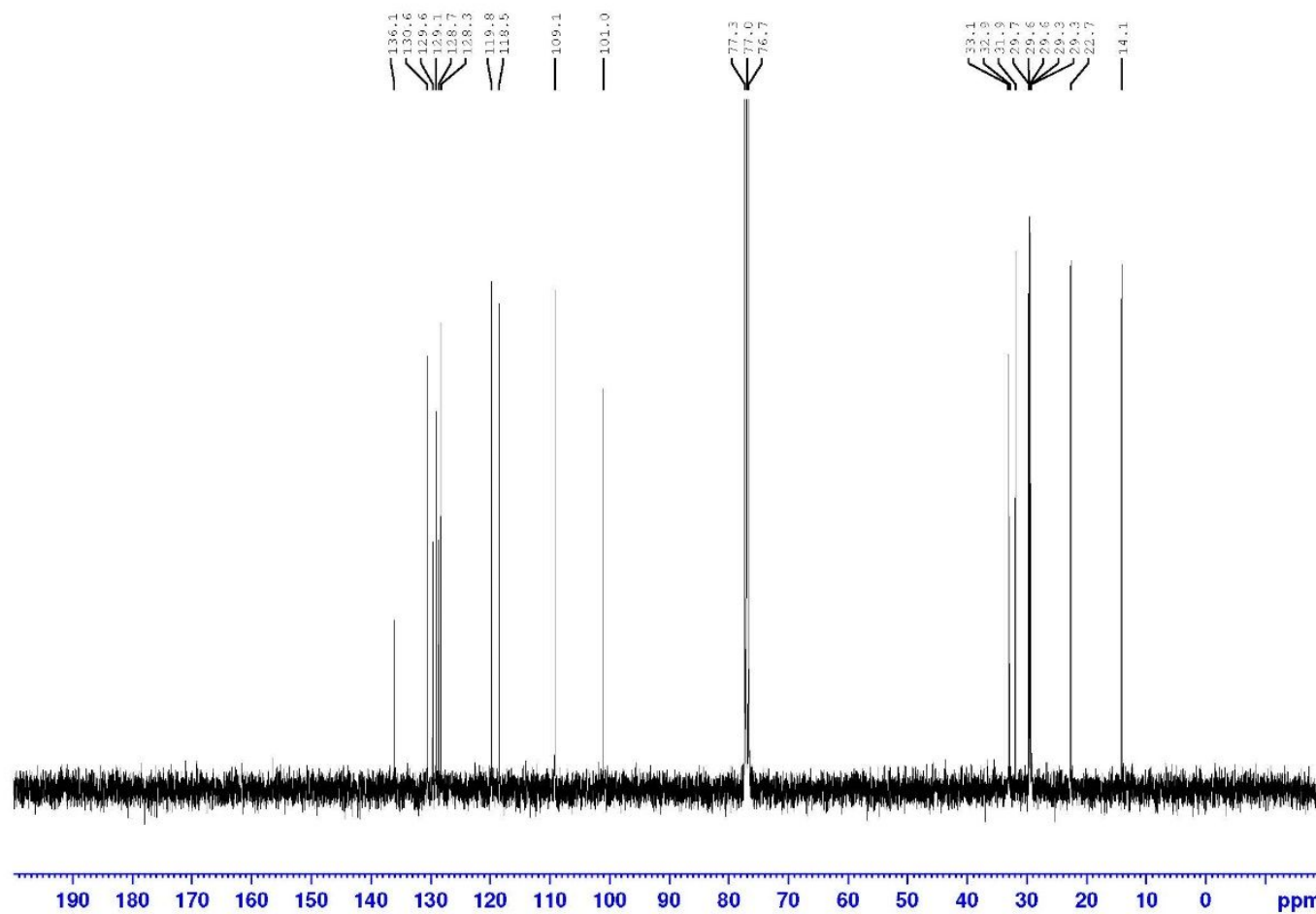

${ }^{1} \mathrm{H}$ NMR Spectrum of $(E)-3 \mathrm{n}, 400 \mathrm{MHz}, \mathrm{CDCl}_{3}$

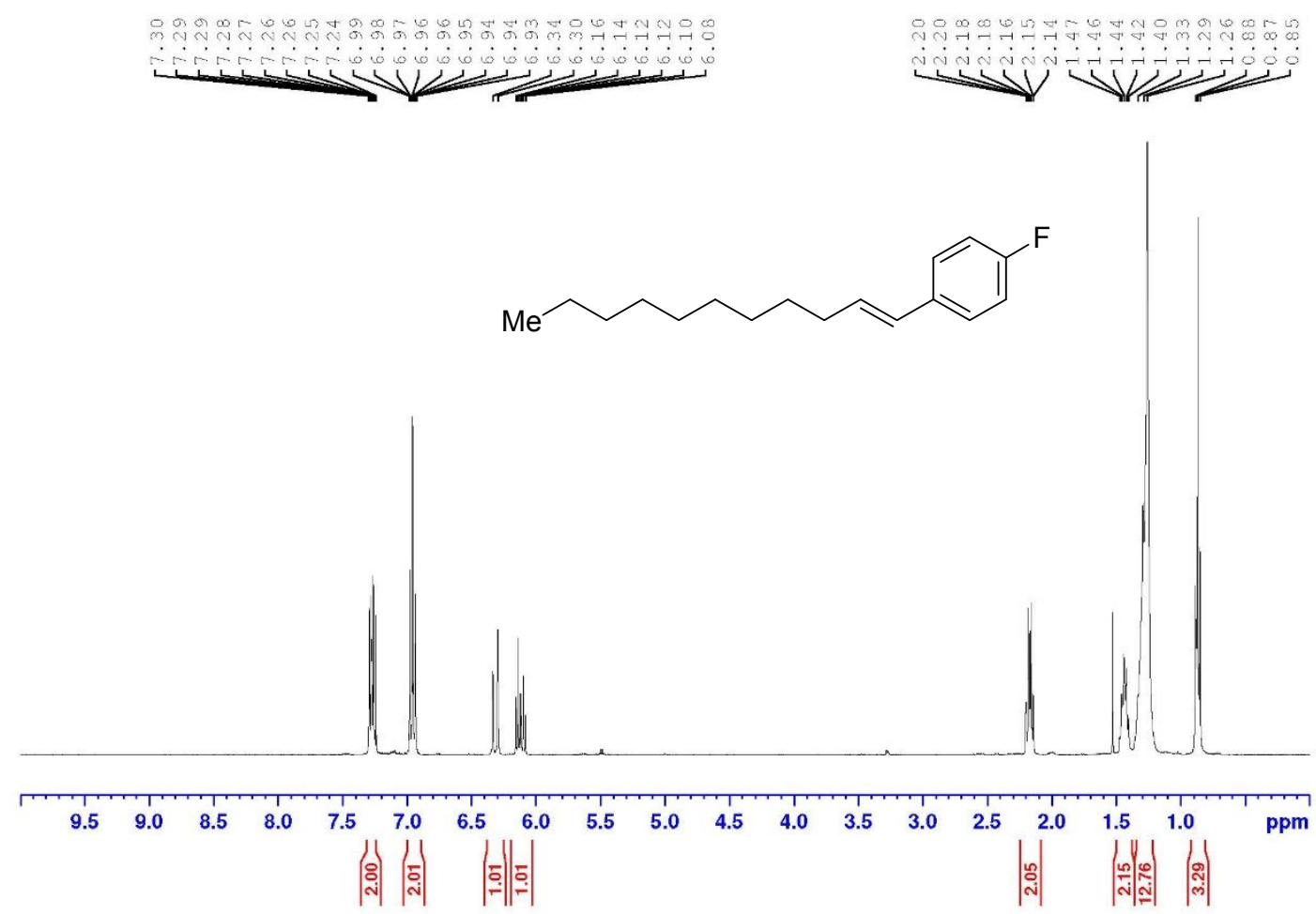

${ }^{13} \mathrm{C}$ NMR Spectrum of (E)-3n, $100 \mathrm{MHz}, \mathrm{CDCl}_{3}$ 


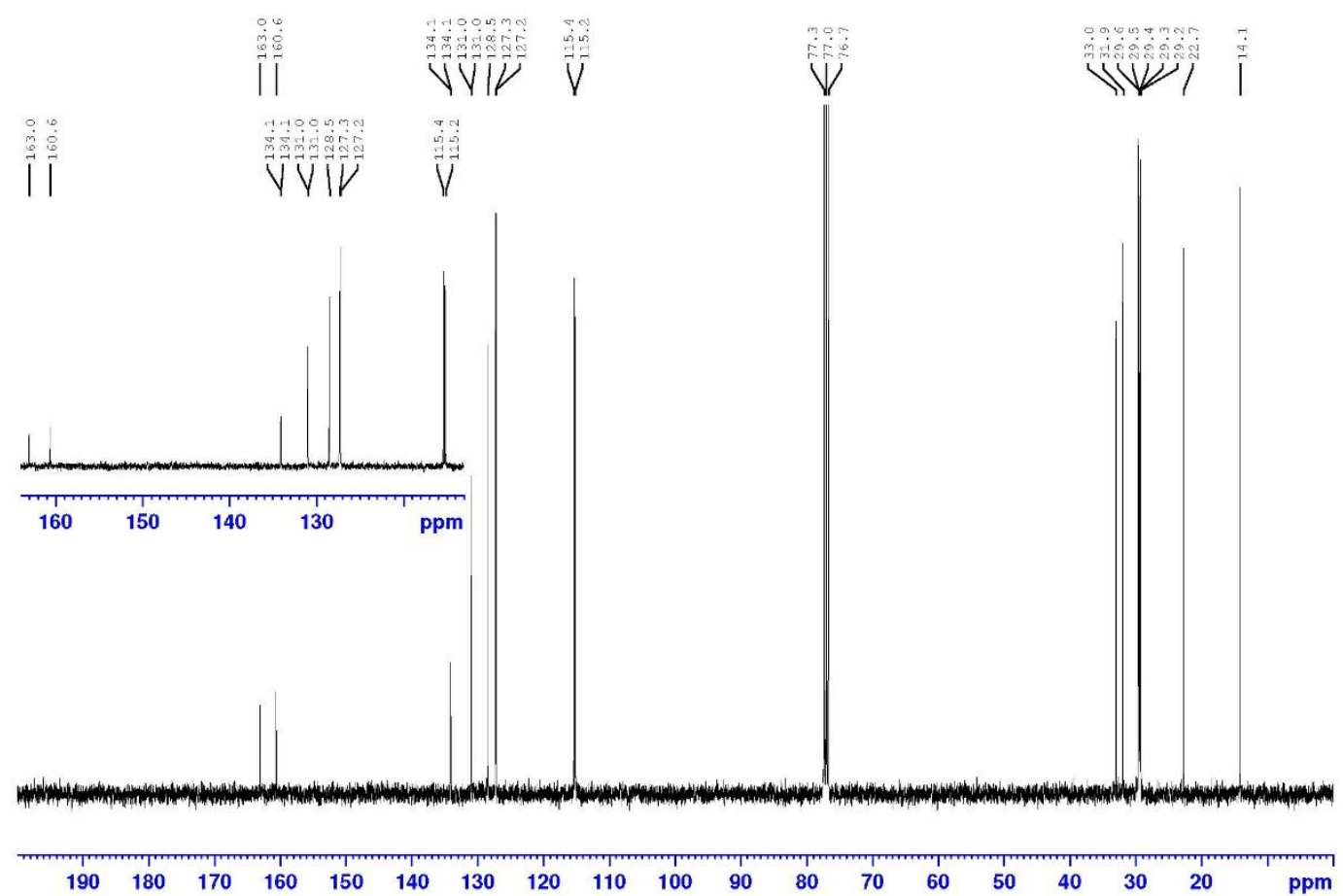

${ }^{1} \mathrm{H}$ NMR Spectrum of $(E)$ - and (Z)-30, $400 \mathrm{MHz}, \mathrm{CDCl}_{3}$

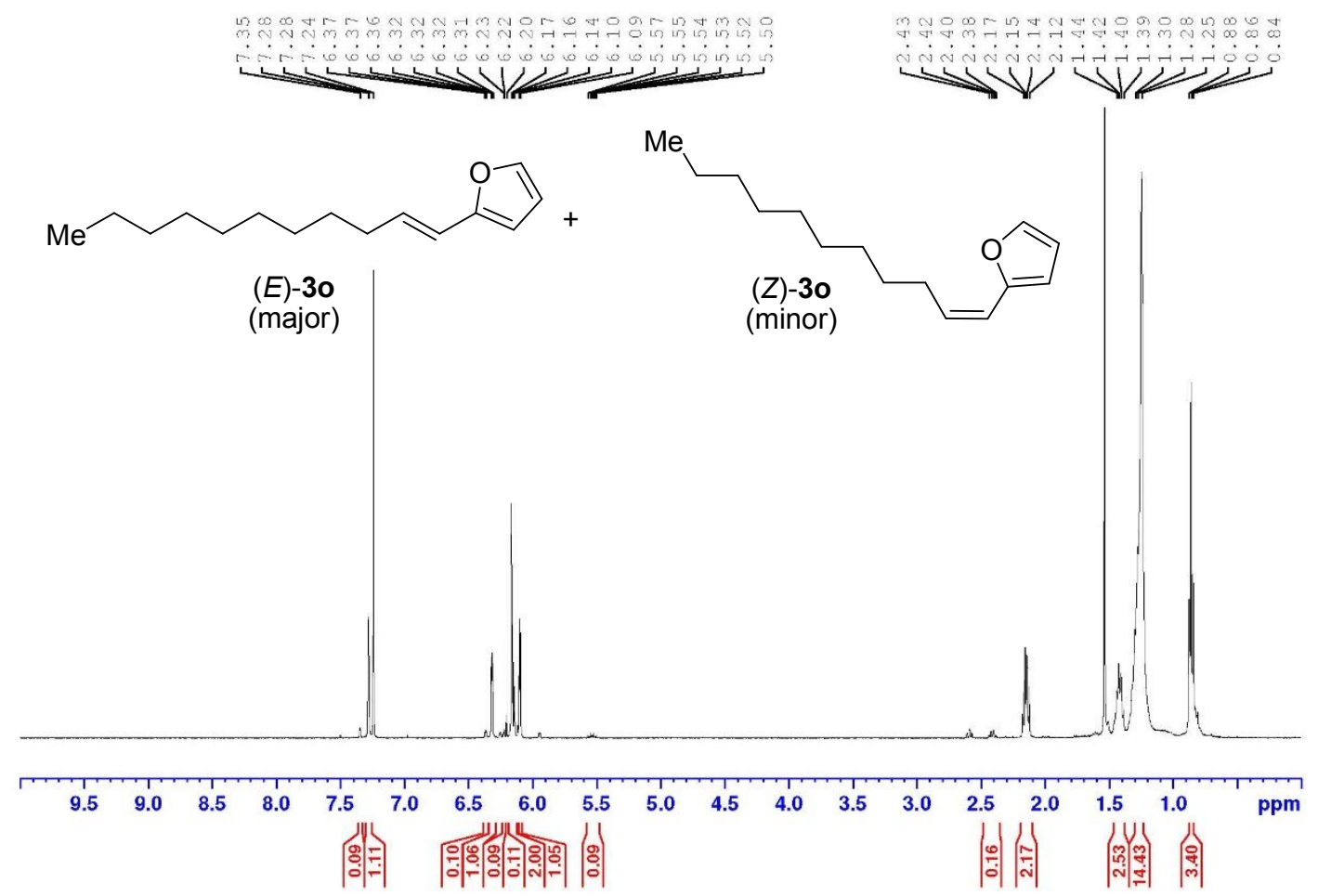

${ }^{13} \mathrm{C}$ NMR Spectrum of $(E)$ - and (Z)-30, $100 \mathrm{MHz}, \mathrm{CDCl}_{3}$ 


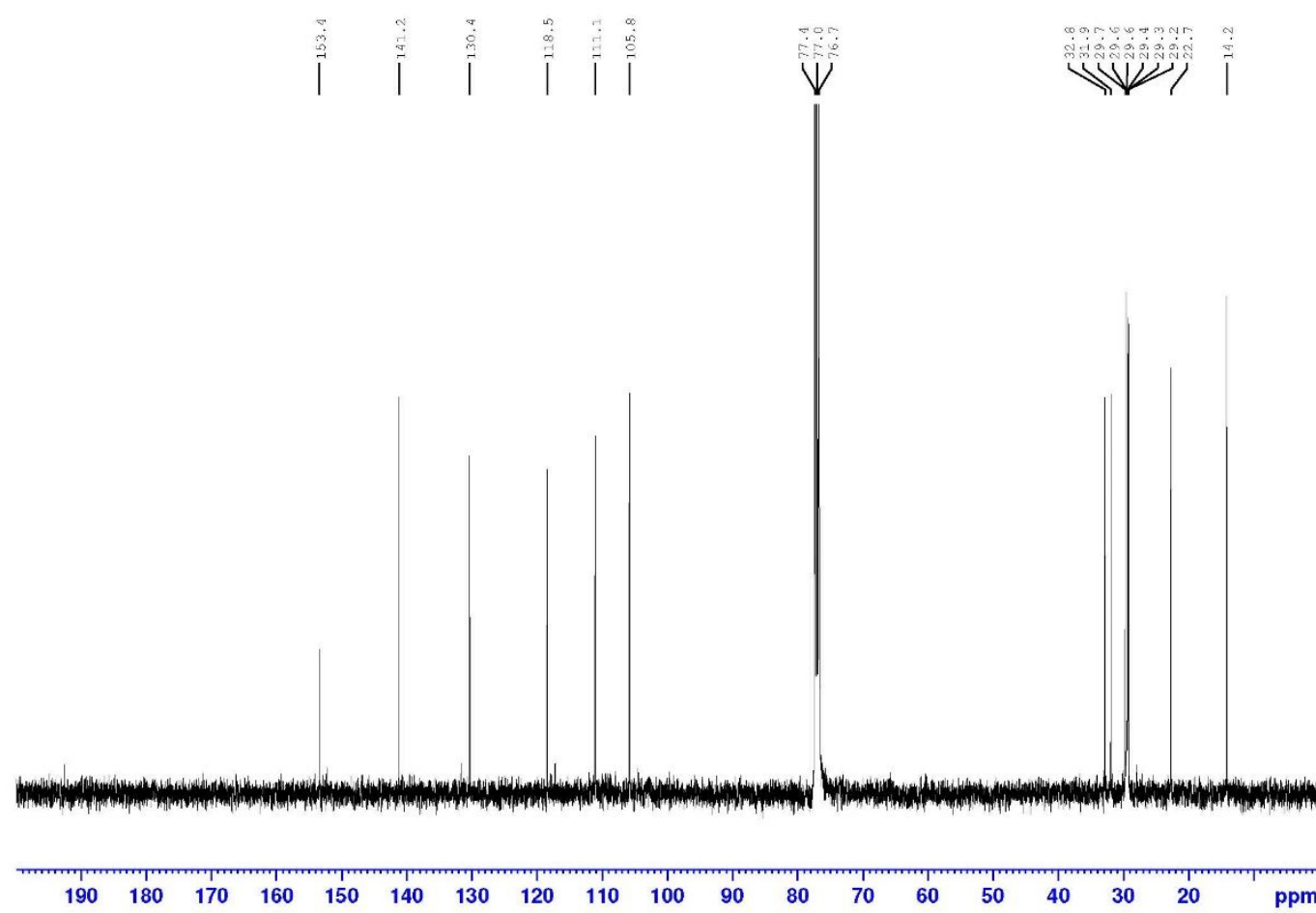

${ }^{1} \mathrm{H}$ NMR Spectrum of $(E)-3 p, 400 \mathrm{MHz}, \mathrm{CDCl}_{3}$

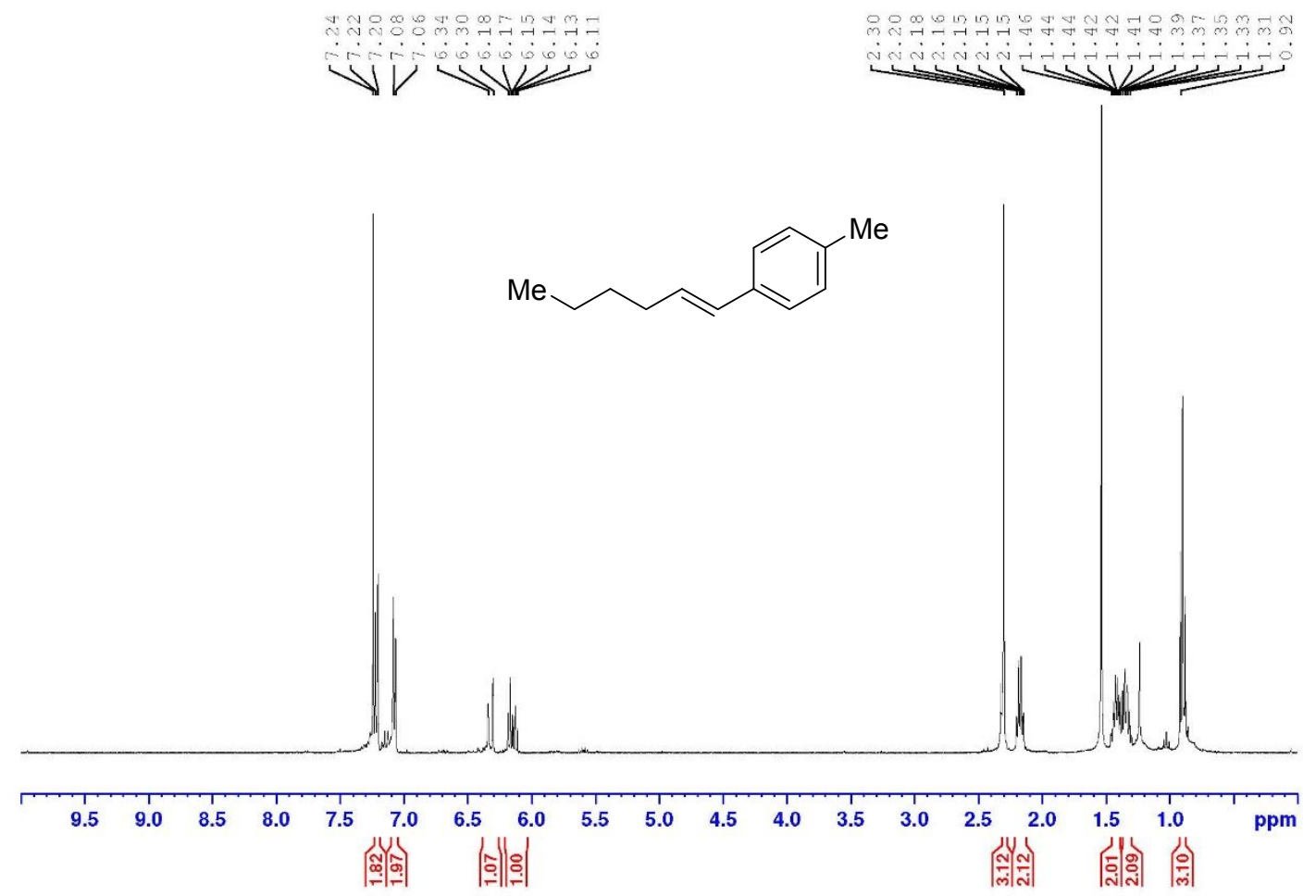

${ }^{13} \mathrm{C}$ NMR Spectrum of (E)-3p, $100 \mathrm{MHz}, \mathrm{CDCl}_{3}$ 

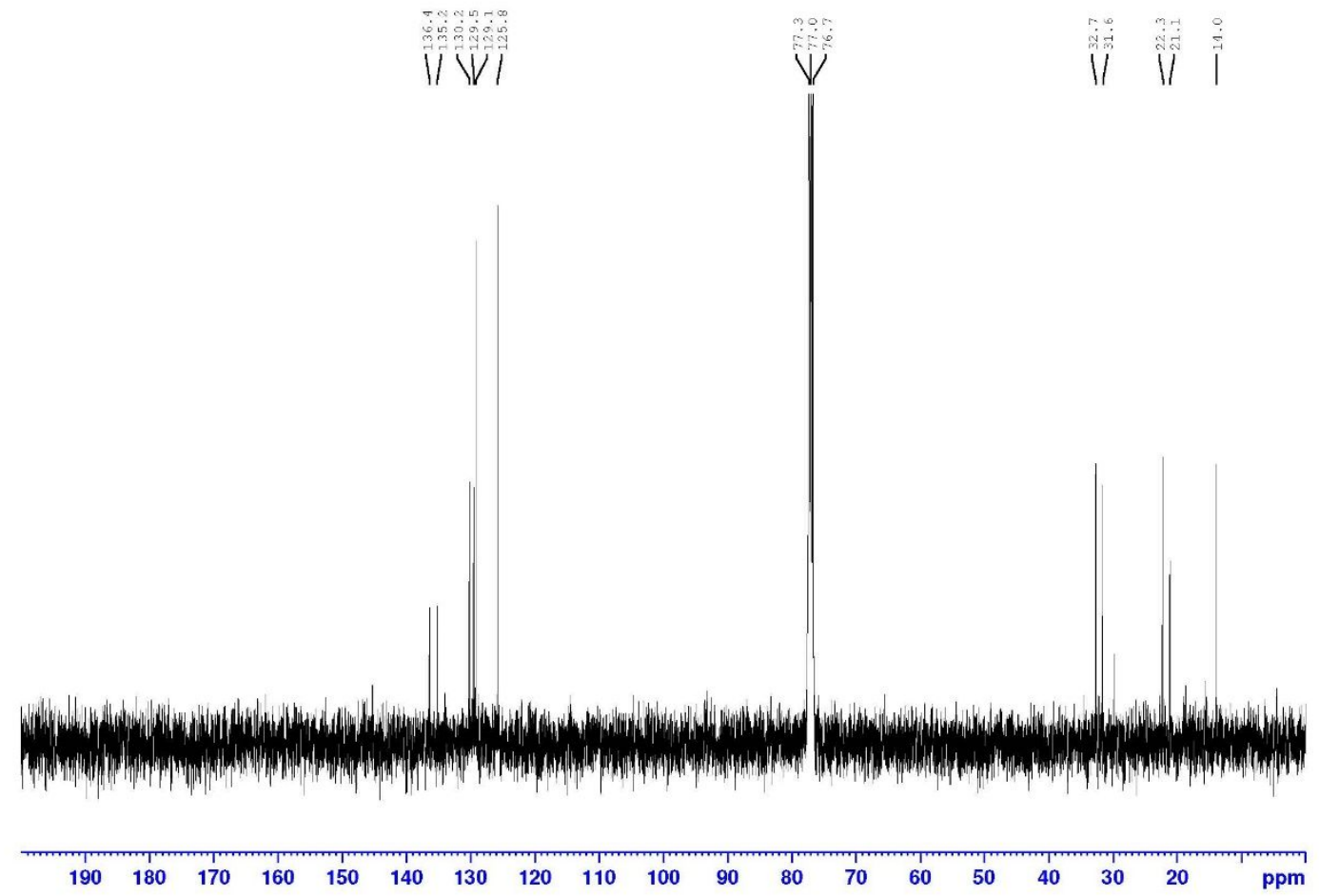

${ }^{1} \mathrm{H}$ NMR Spectrum of (Z)-3p, $400 \mathrm{MHz}, \mathrm{CDCl}_{3}$

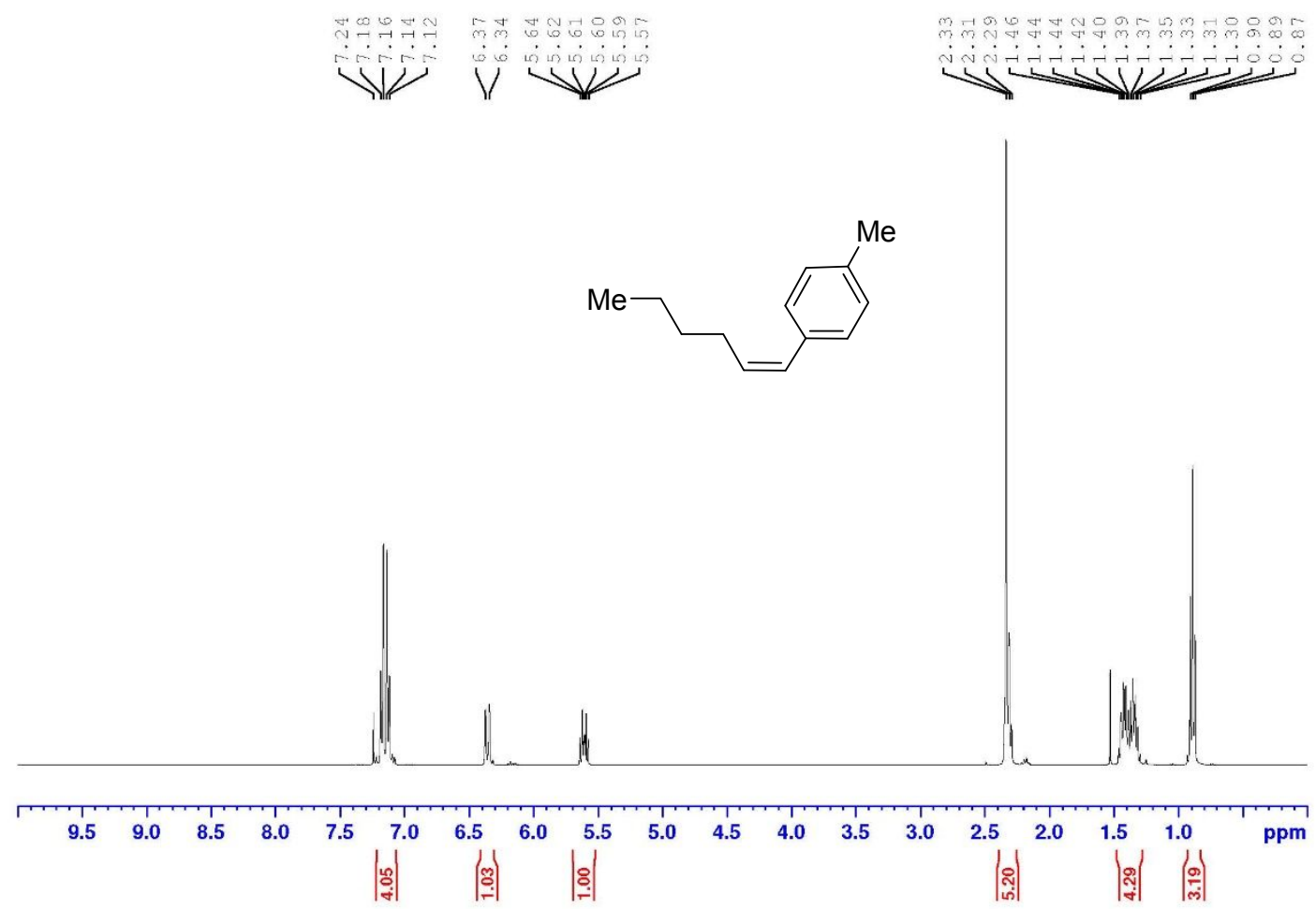

${ }^{13} \mathrm{C}$ NMR Spectrum of (Z)-3p, $100 \mathrm{MHz}, \mathrm{CDCl}_{3}$ 


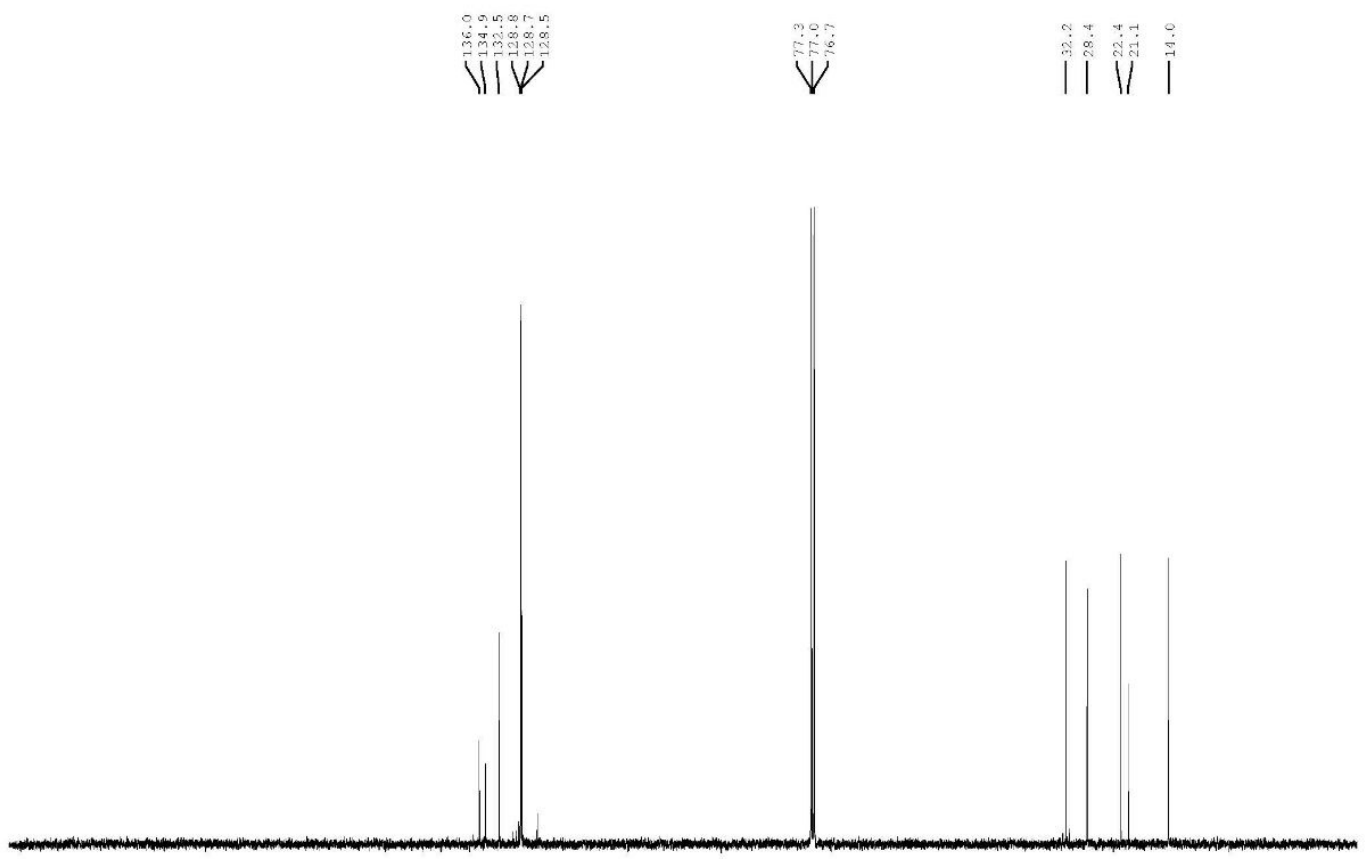

$\begin{array}{lllllllllllllllllllllll}210 & 200 & 190 & 180 & 170 & 160 & 150 & 140 & 130 & 120 & 110 & 100 & 90 & 80 & 70 & 60 & 50 & 40 & 30 & 20 & 10 & 0 & \mathrm{ppm}\end{array}$

${ }^{1} \mathrm{H}$ NMR Spectrum of $(E)$ - and (Z)-3q, $400 \mathrm{MHz}, \mathrm{CDCl}_{3}$

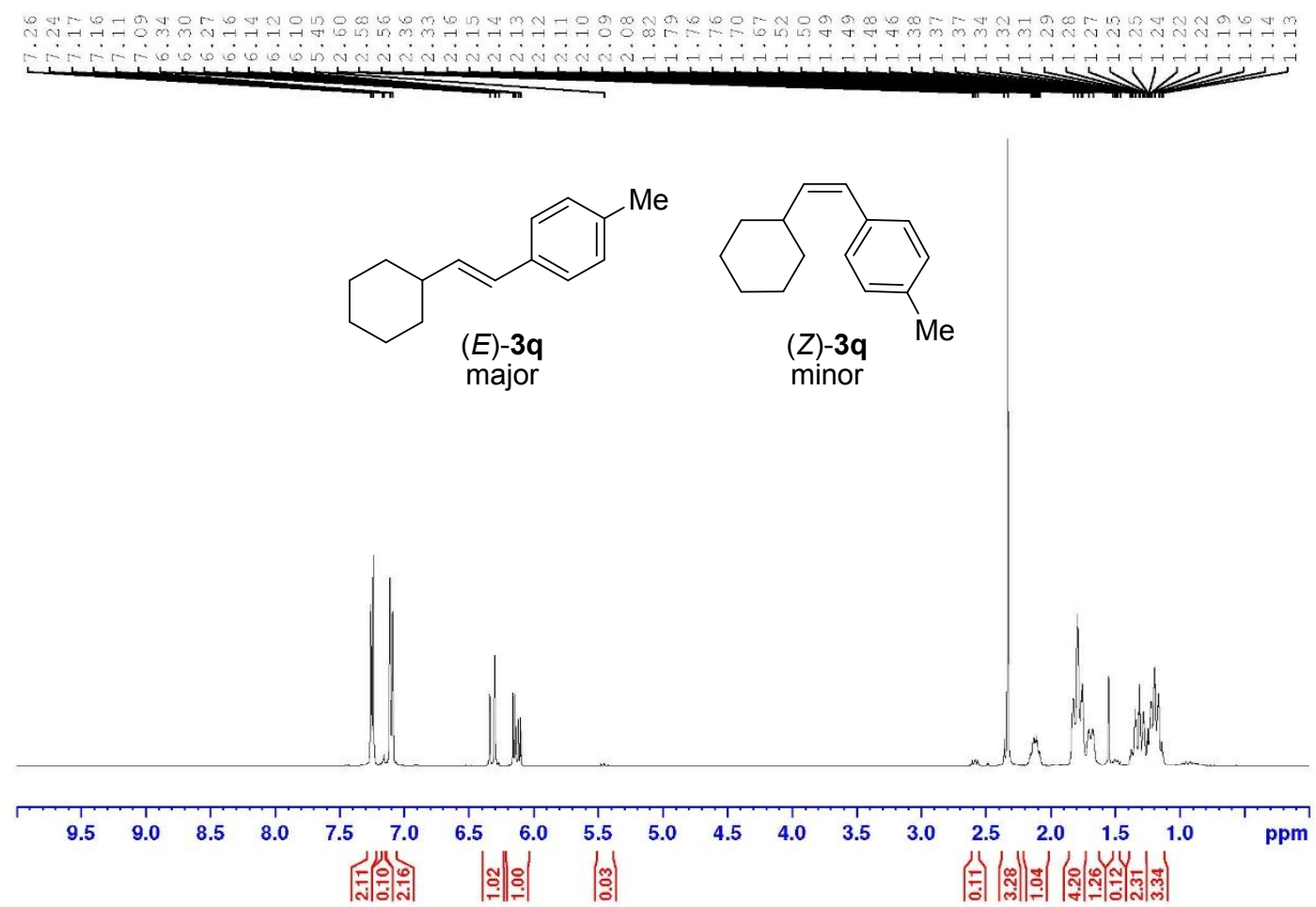

${ }^{13} \mathrm{C}$ NMR Spectrum of $(E)$ - and (Z)-3q, $100 \mathrm{MHz}, \mathrm{CDCl}_{3}$ 


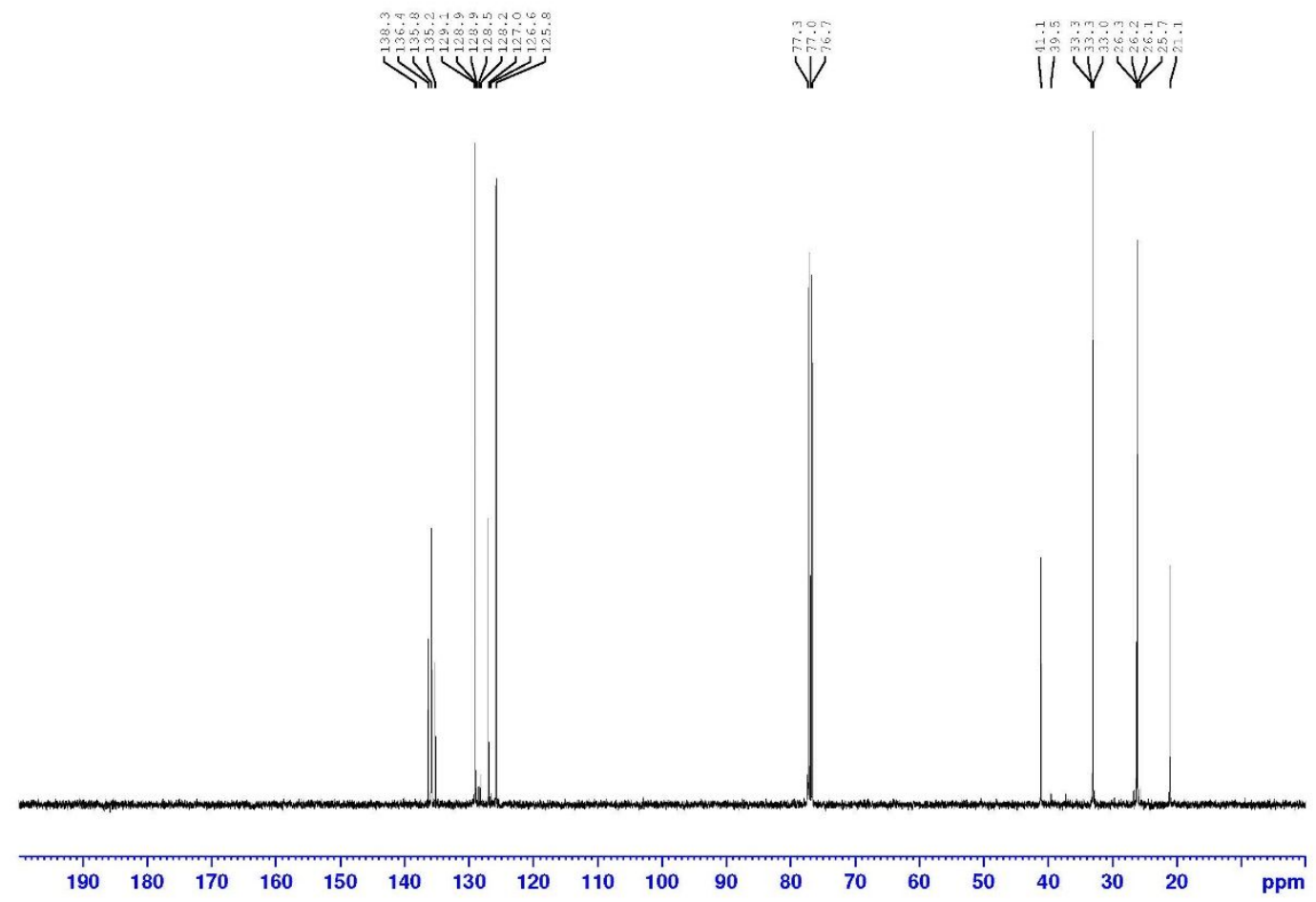

${ }^{1} \mathrm{H}$ NMR Spectrum of $3 r, 400 \mathrm{MHz}, \mathrm{CDCl}_{3}$

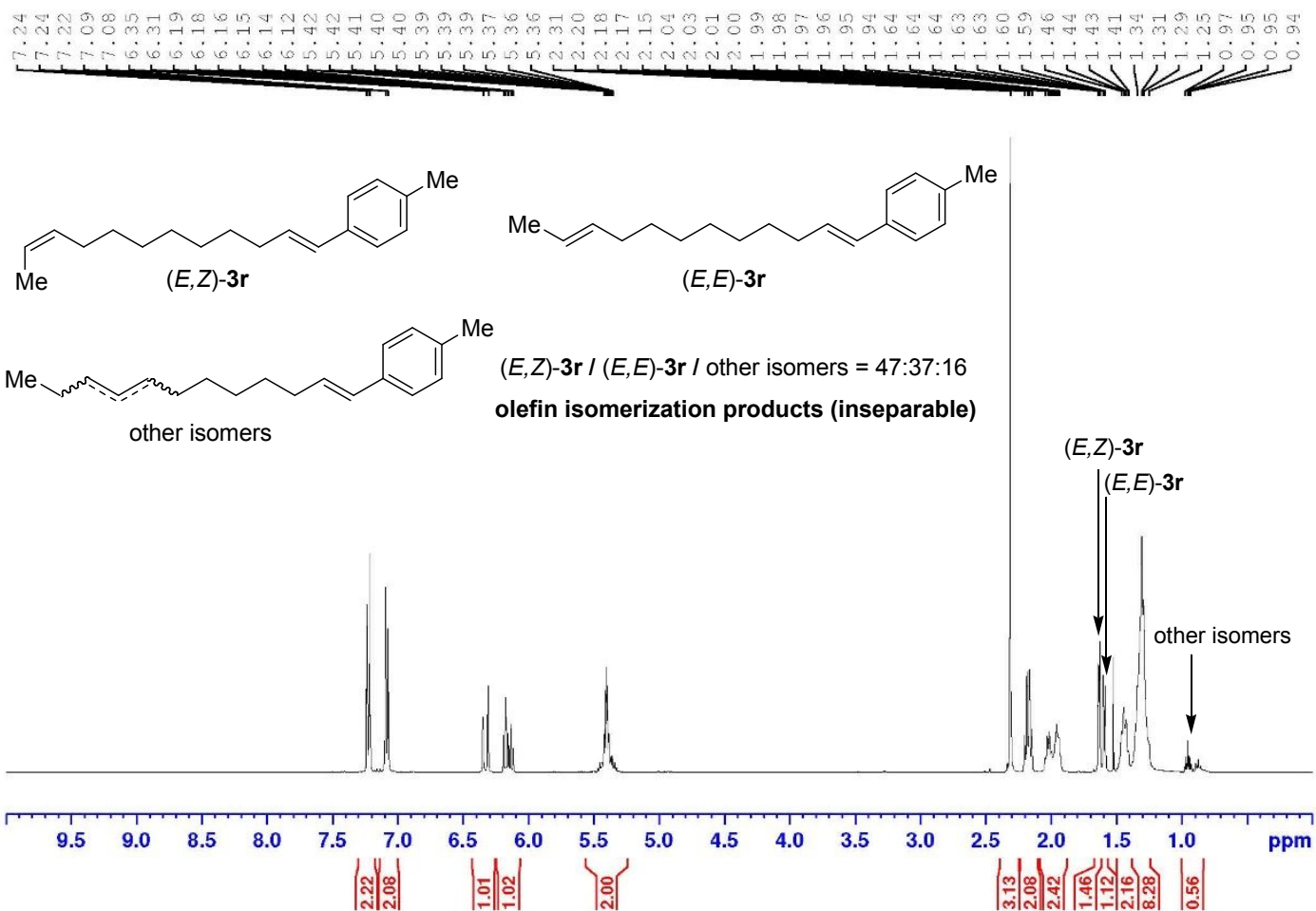

${ }^{13} \mathrm{C}$ NMR Spectrum of $3 \mathrm{r}, 100 \mathrm{MHz}, \mathrm{CDCl}_{3}$ 


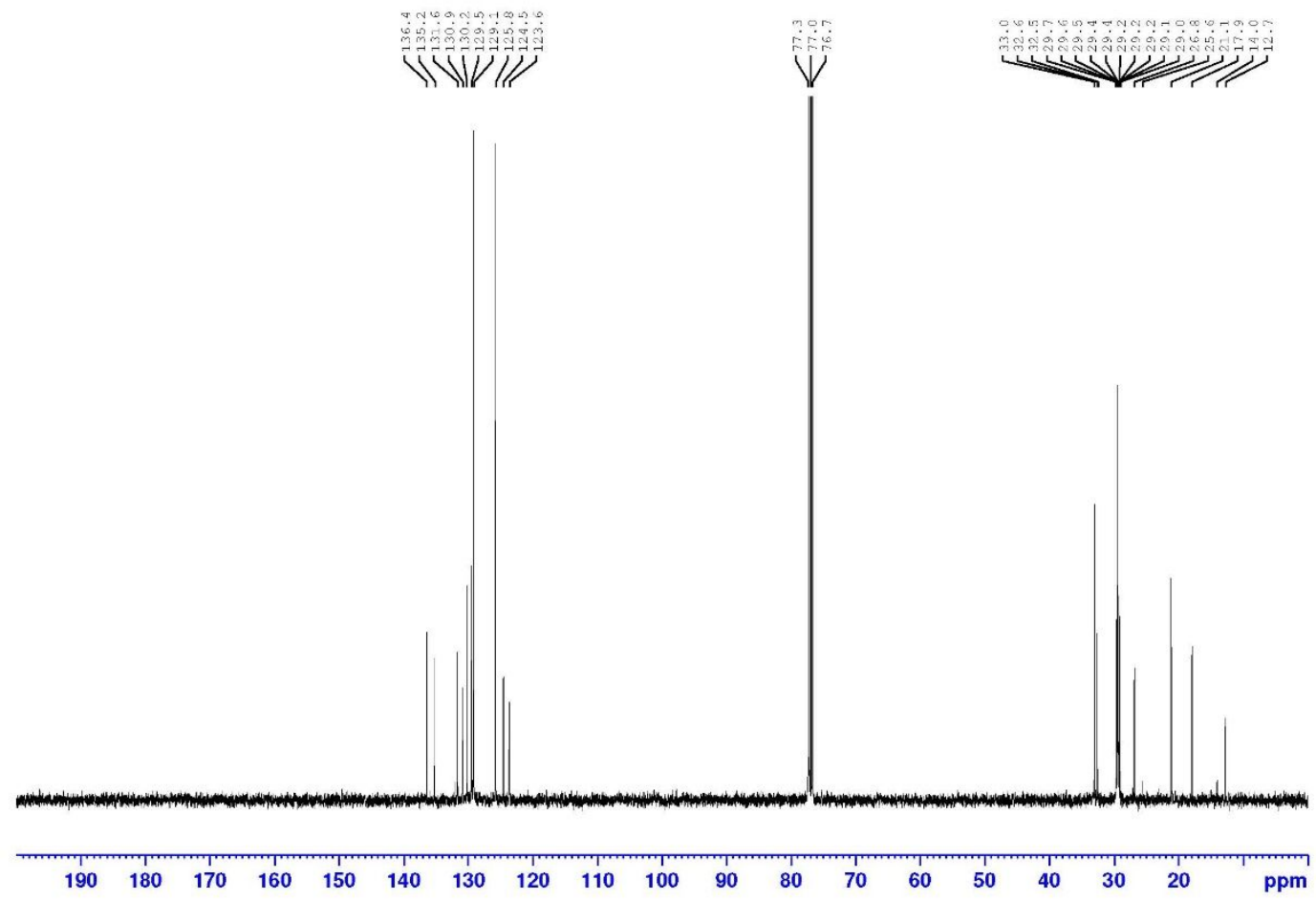

${ }^{1} \mathrm{H}$ NMR Spectrum of $(E)-3 s, 400 \mathrm{MHz}, \mathrm{CDCl}_{3}$

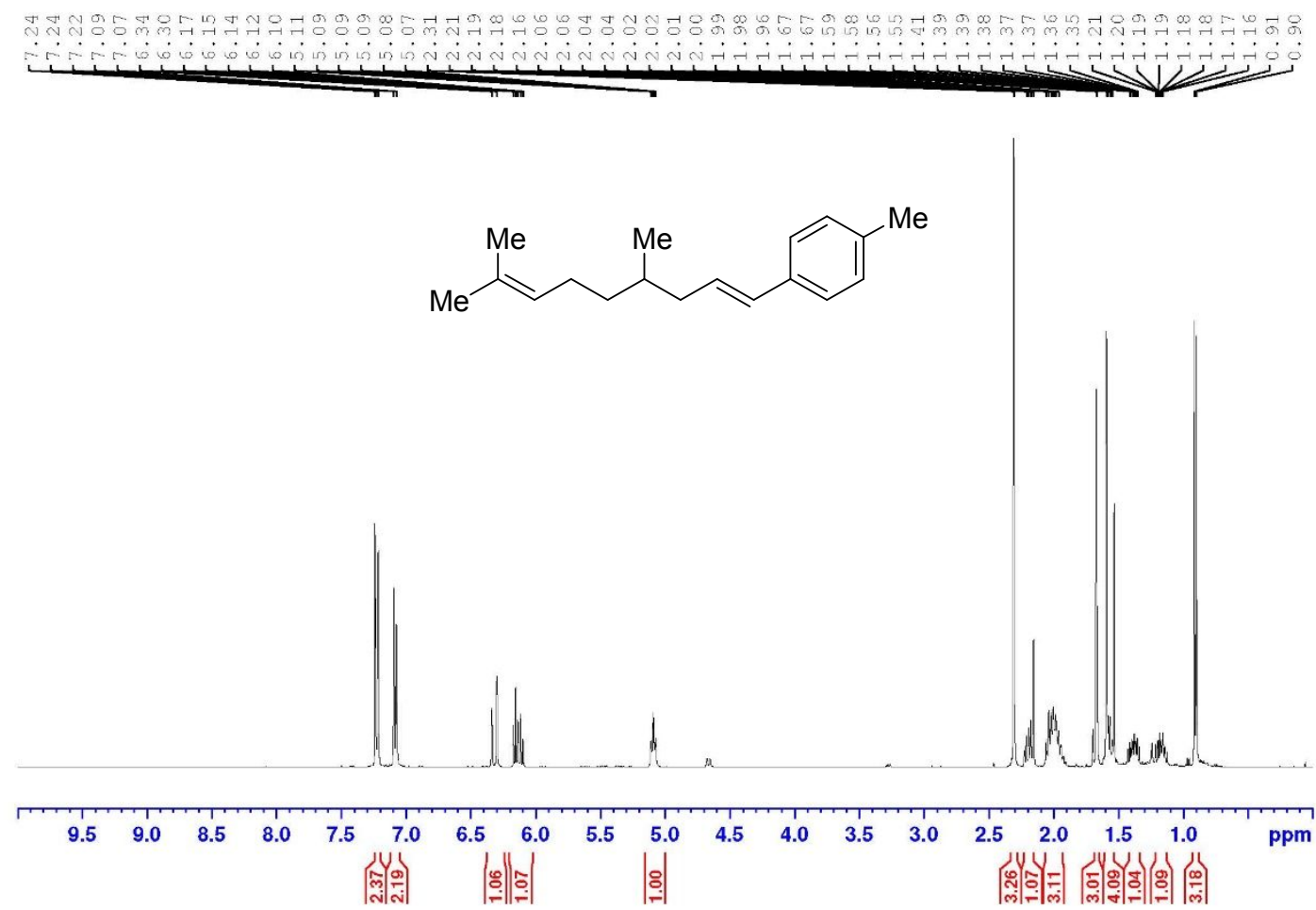

${ }^{13} \mathrm{C}$ NMR Spectrum of (E)-3s, $100 \mathrm{MHz}, \mathrm{CDCl}_{3}$ 


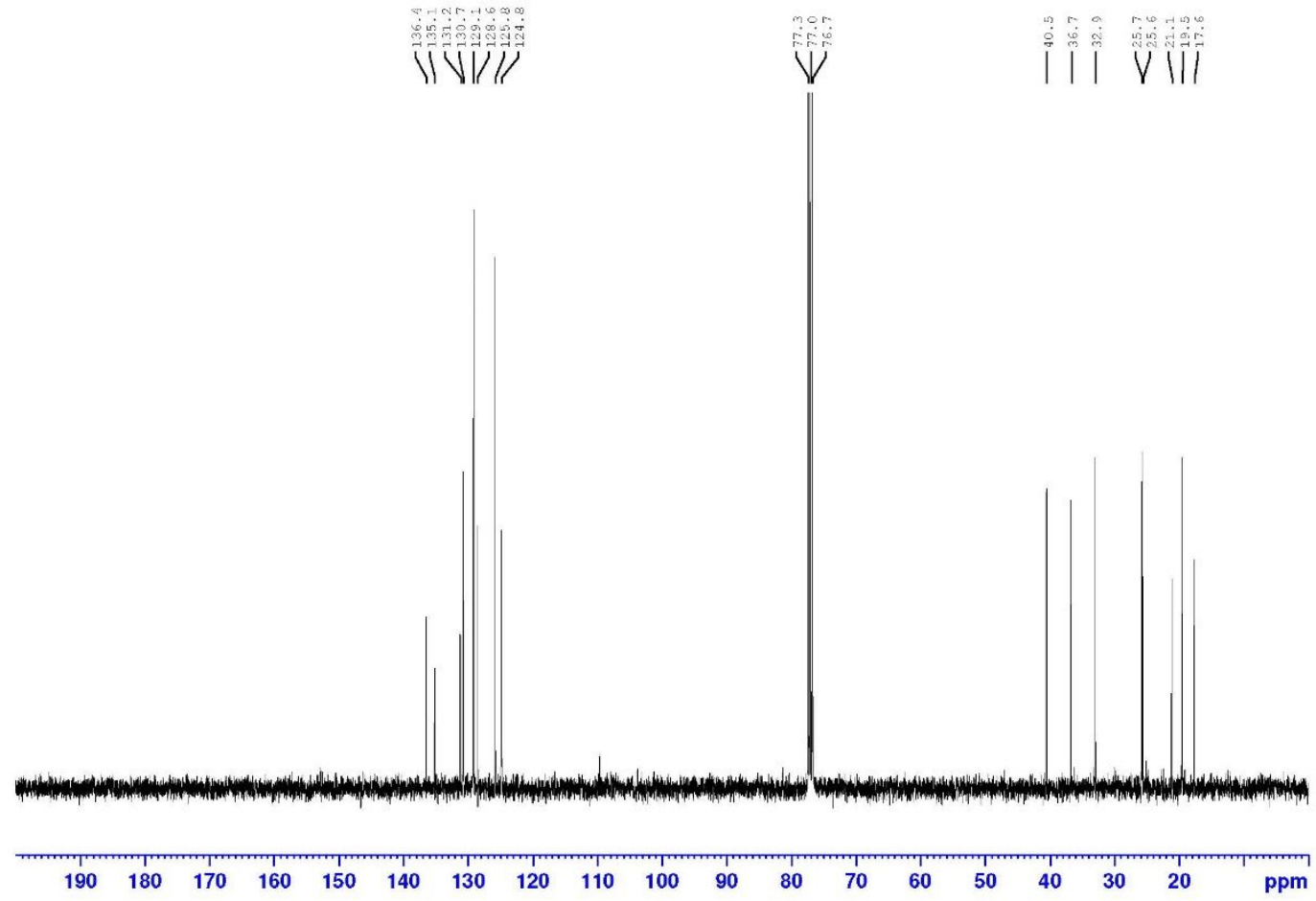

${ }^{1} \mathrm{H}$ NMR Spectrum of $(E)-3 \mathbf{t}, 400 \mathrm{MHz}, \mathrm{CDCl}_{3}$
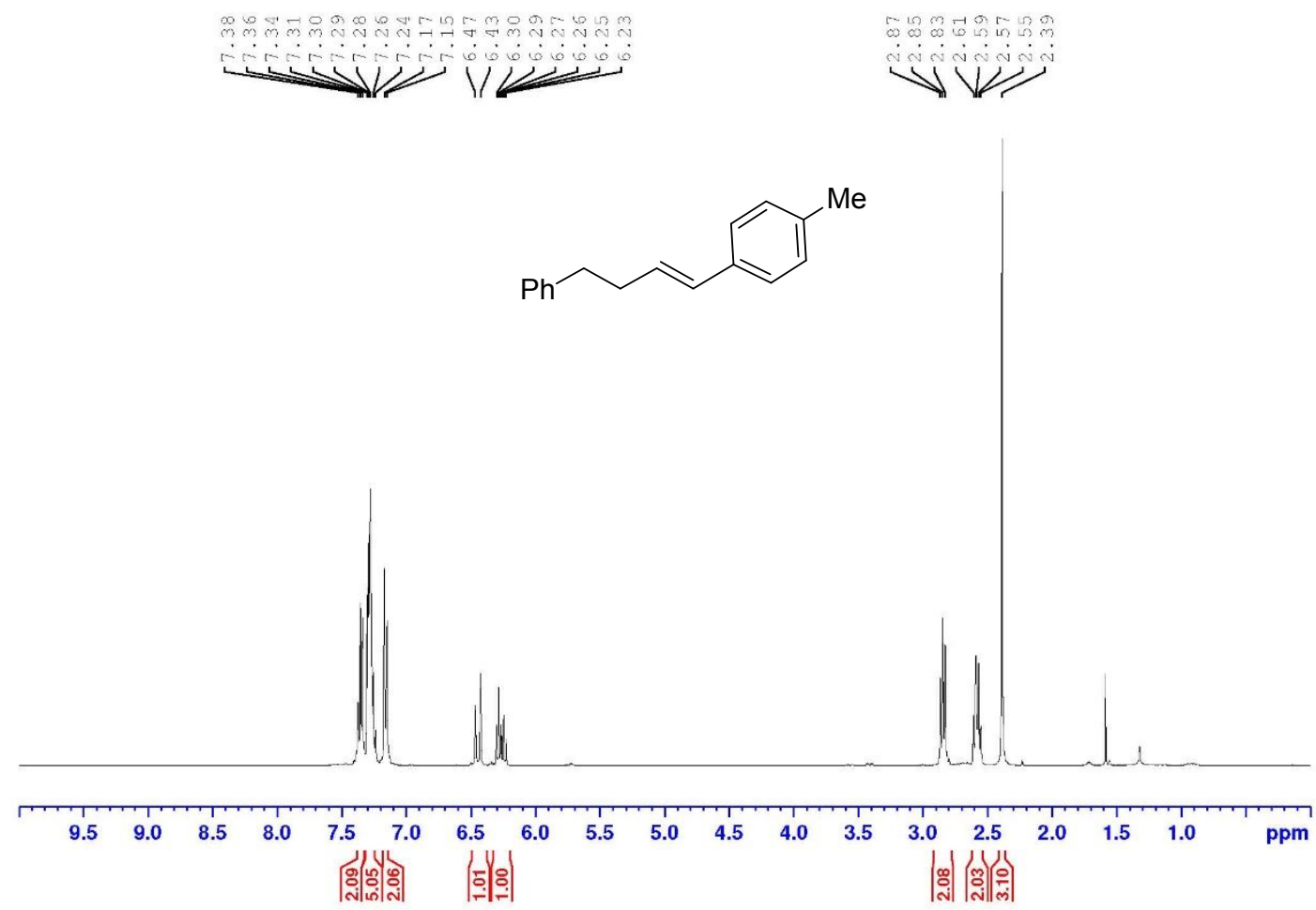

${ }^{13} \mathrm{C}$ NMR Spectrum of (E)-3t, $100 \mathrm{MHz}, \mathrm{CDCl}_{3}$ 


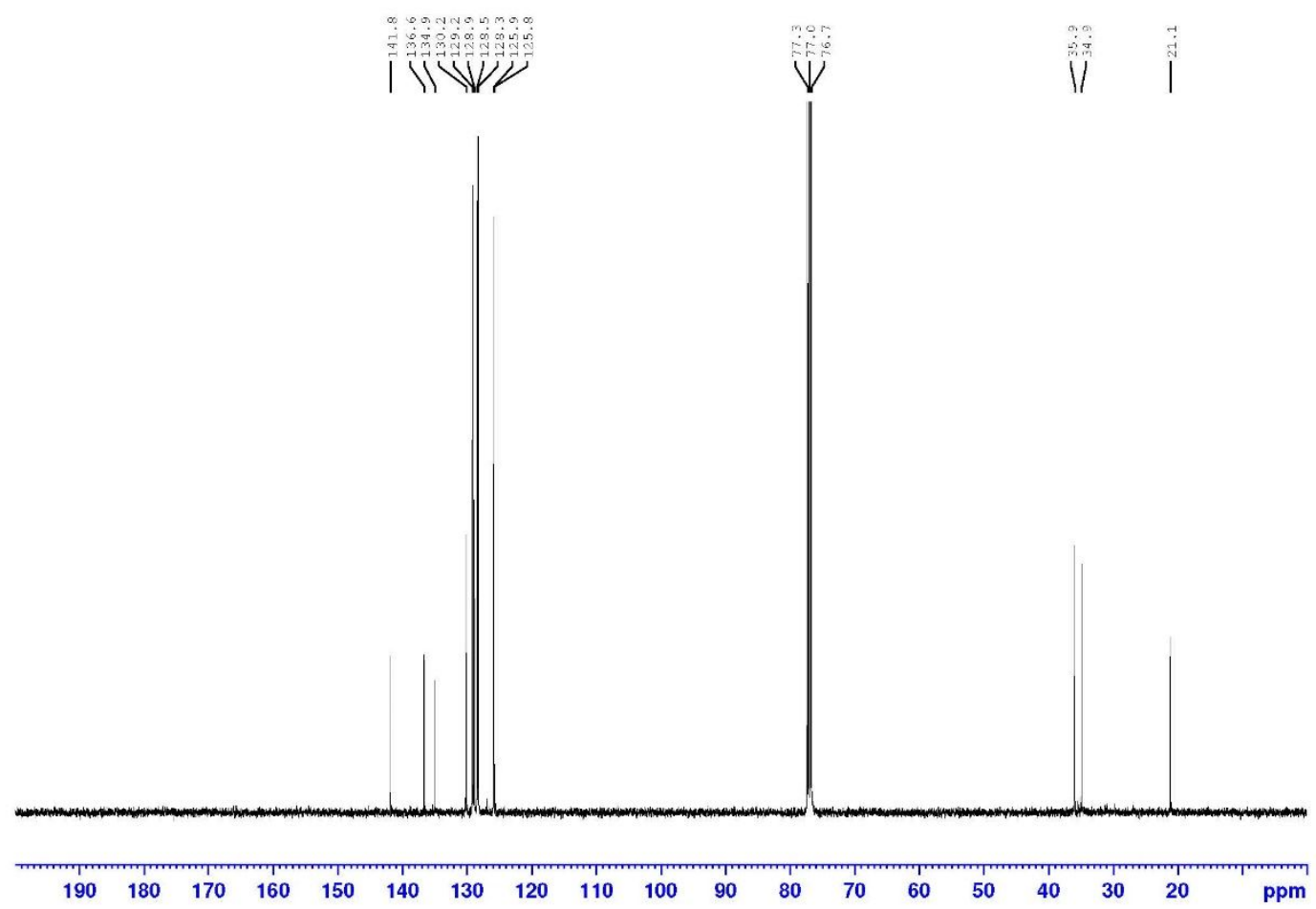

${ }^{1} \mathrm{H}$ NMR Spectrum of $(E)-3 \mathbf{u}$ and $(E)-3 \mathbf{u}^{\prime}, 400 \mathrm{MHz}, \mathrm{CDCl}_{3}$

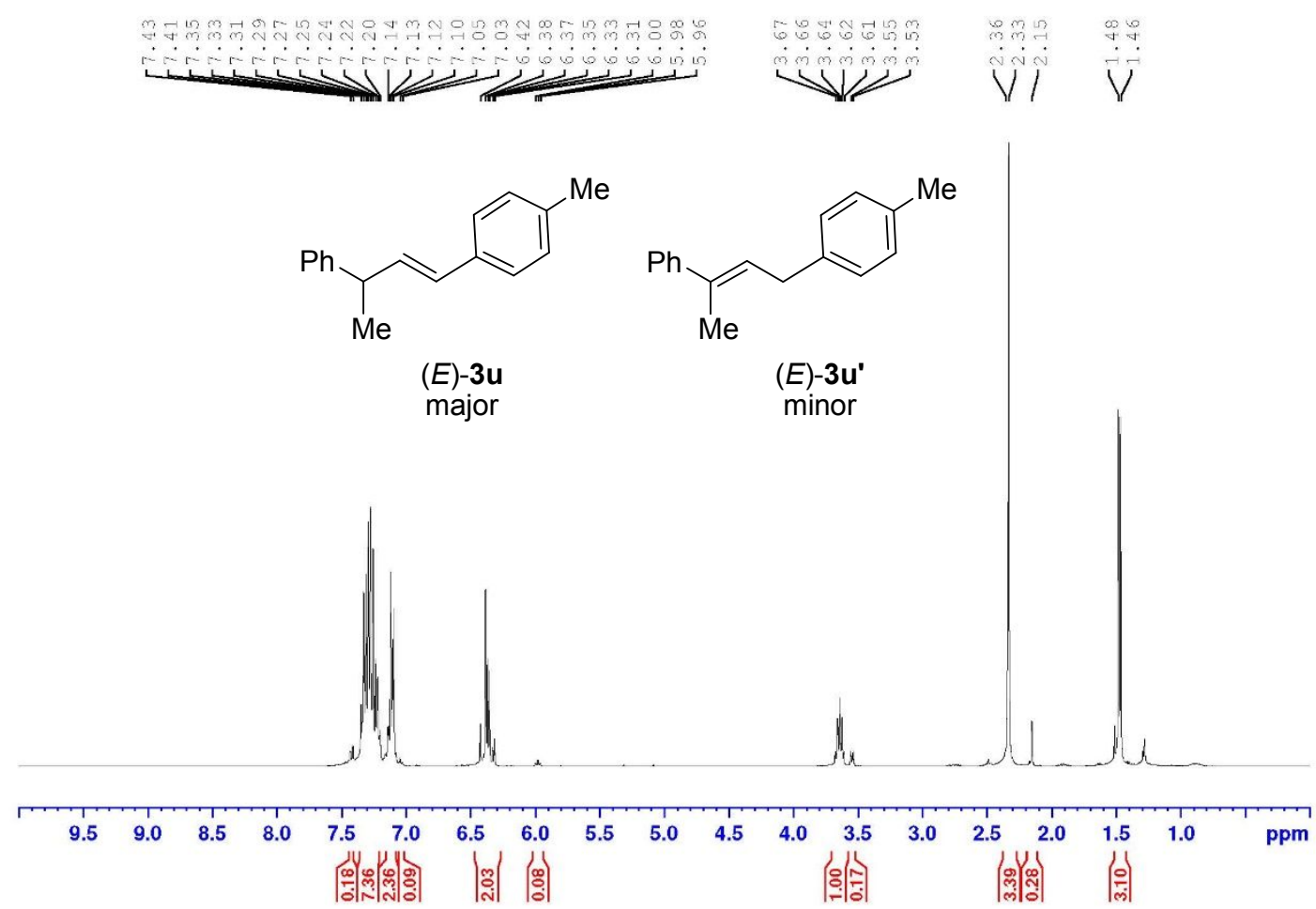

${ }^{13} \mathrm{C}$ NMR Spectrum of $(E)-\mathbf{3 u}$ and $(E)-\mathbf{3} \mathbf{u}^{\prime}, 100 \mathrm{MHz}, \mathrm{CDCl}_{3}$ 


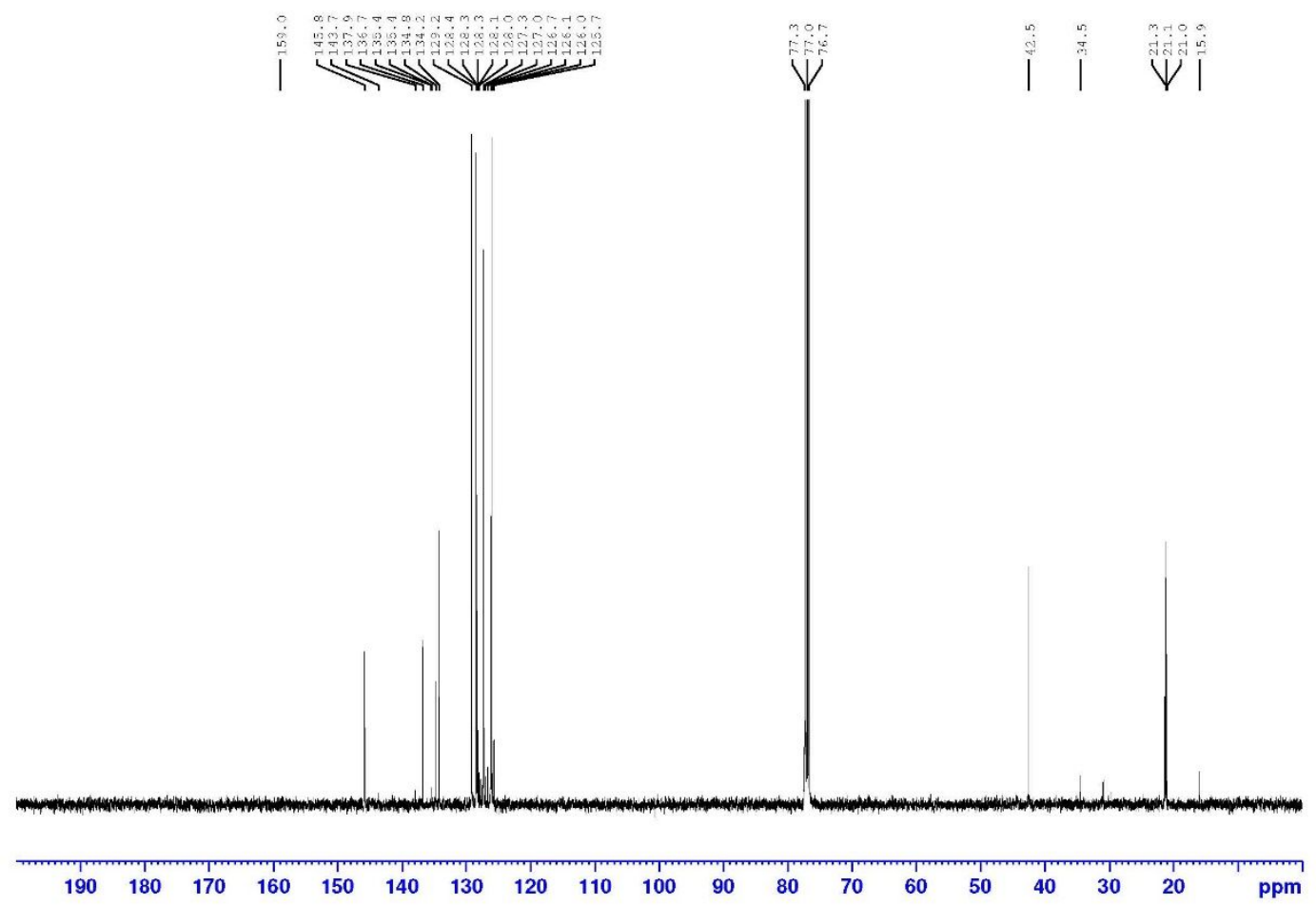

${ }^{1} \mathrm{H}$ NMR Spectrum of $(E)-3 v, 400 \mathrm{MHz}, \mathrm{CDCl}_{3}$
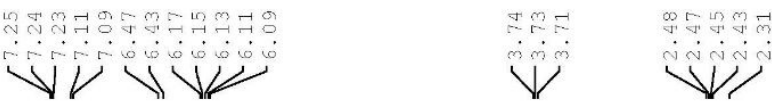<smiles>Cc1ccc(/C=C/CCO)cc1</smiles>

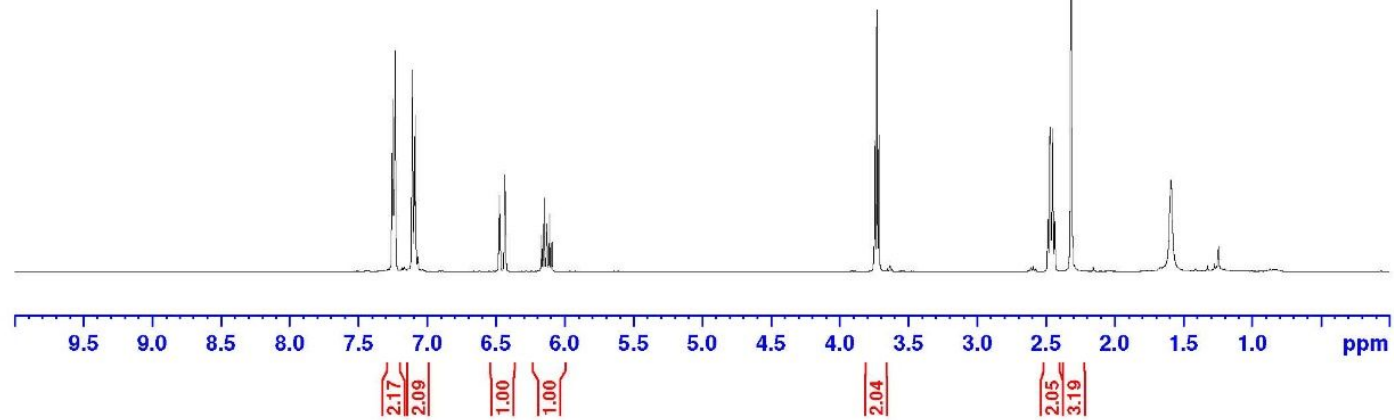

${ }^{13} \mathrm{C}$ NMR Spectrum of $(E)-\mathbf{3 v}, 100 \mathrm{MHz}, \mathrm{CDCl}_{3}$ 


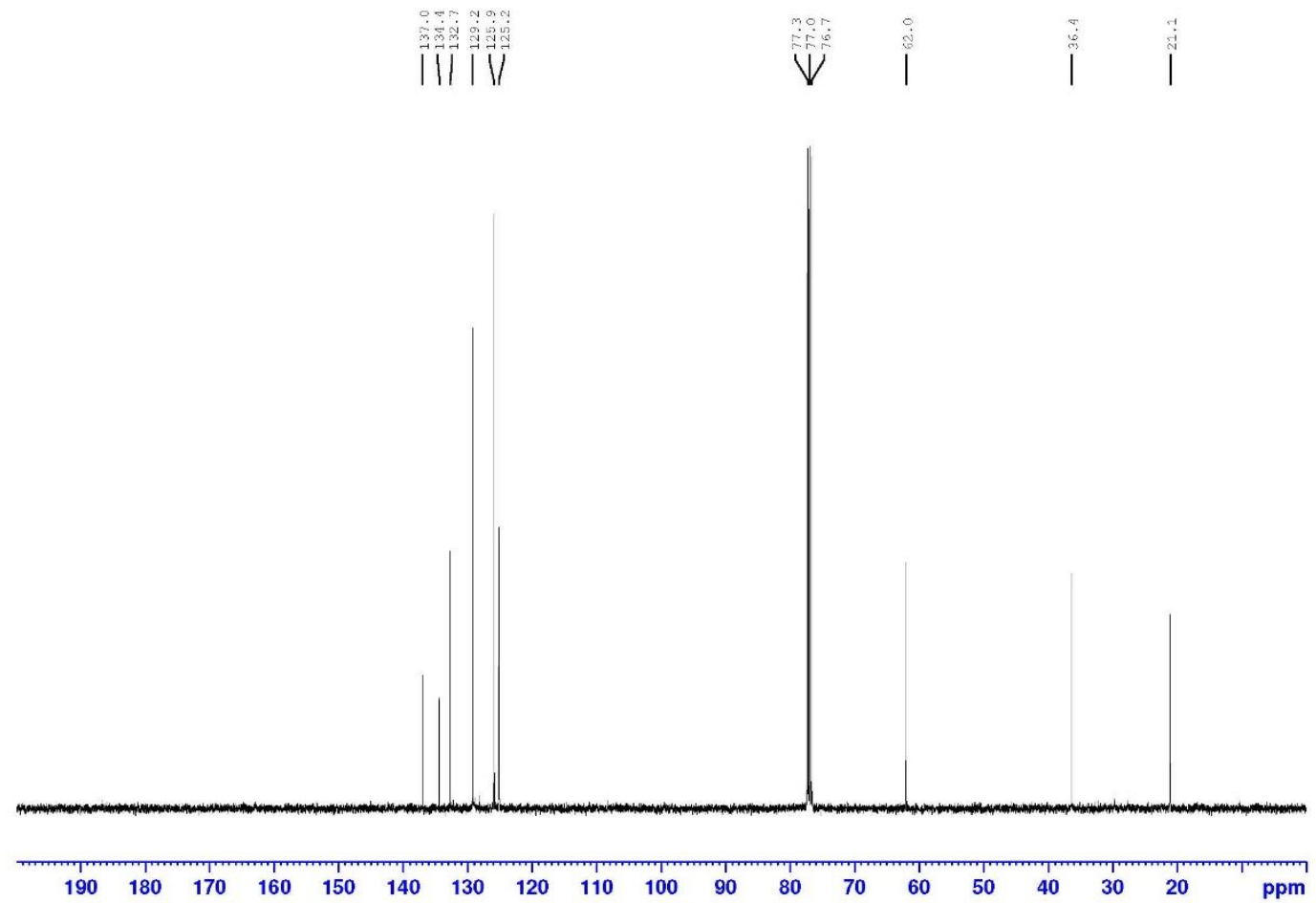

${ }^{1} \mathrm{H}$ NMR Spectrum of (Z)-3v, $400 \mathrm{MHz}, \mathrm{CDCl}_{3}$

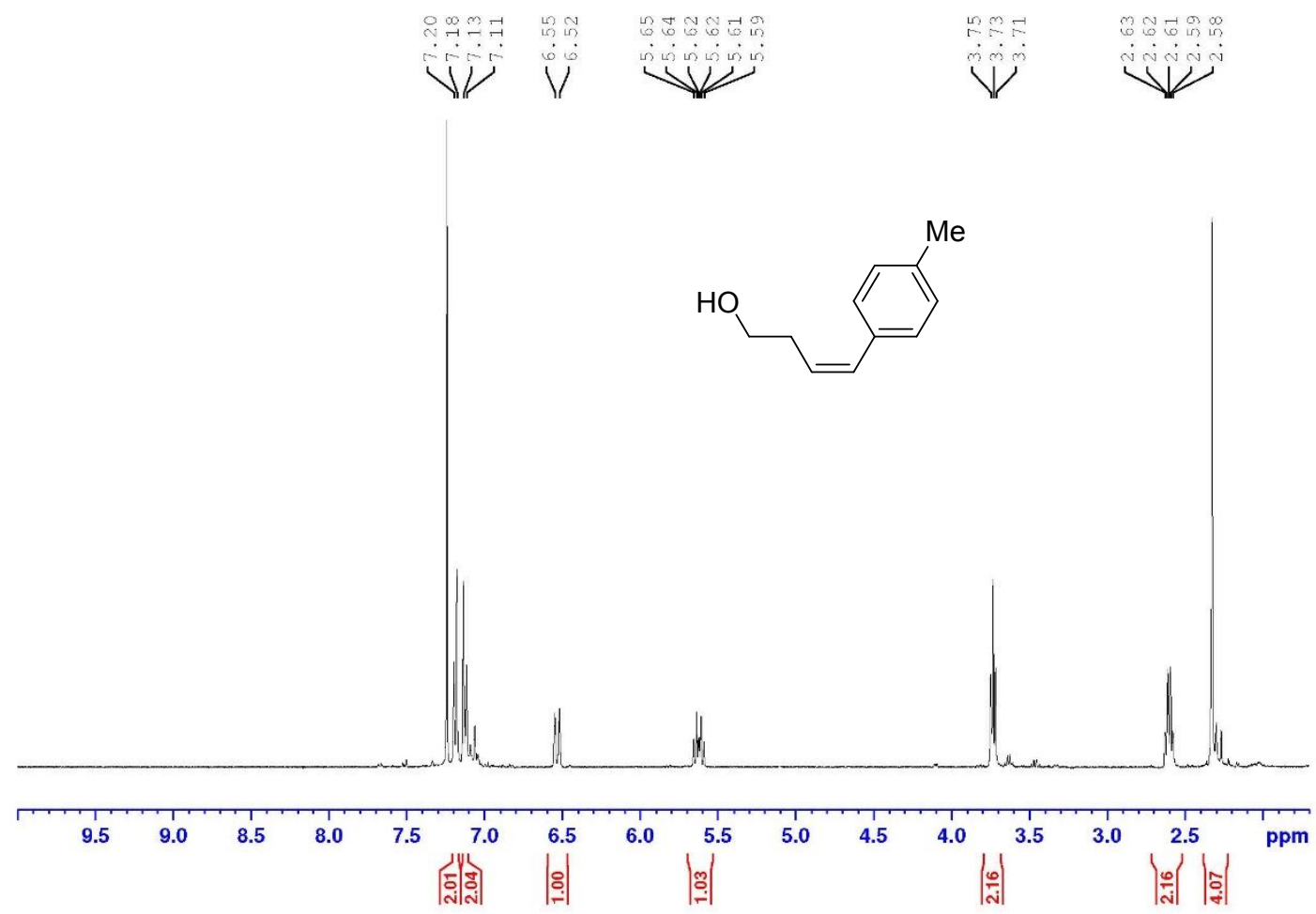

${ }^{13} \mathrm{C}$ NMR Spectrum of (Z)-3v, $100 \mathrm{MHz}, \mathrm{CDCl}_{3}$ 


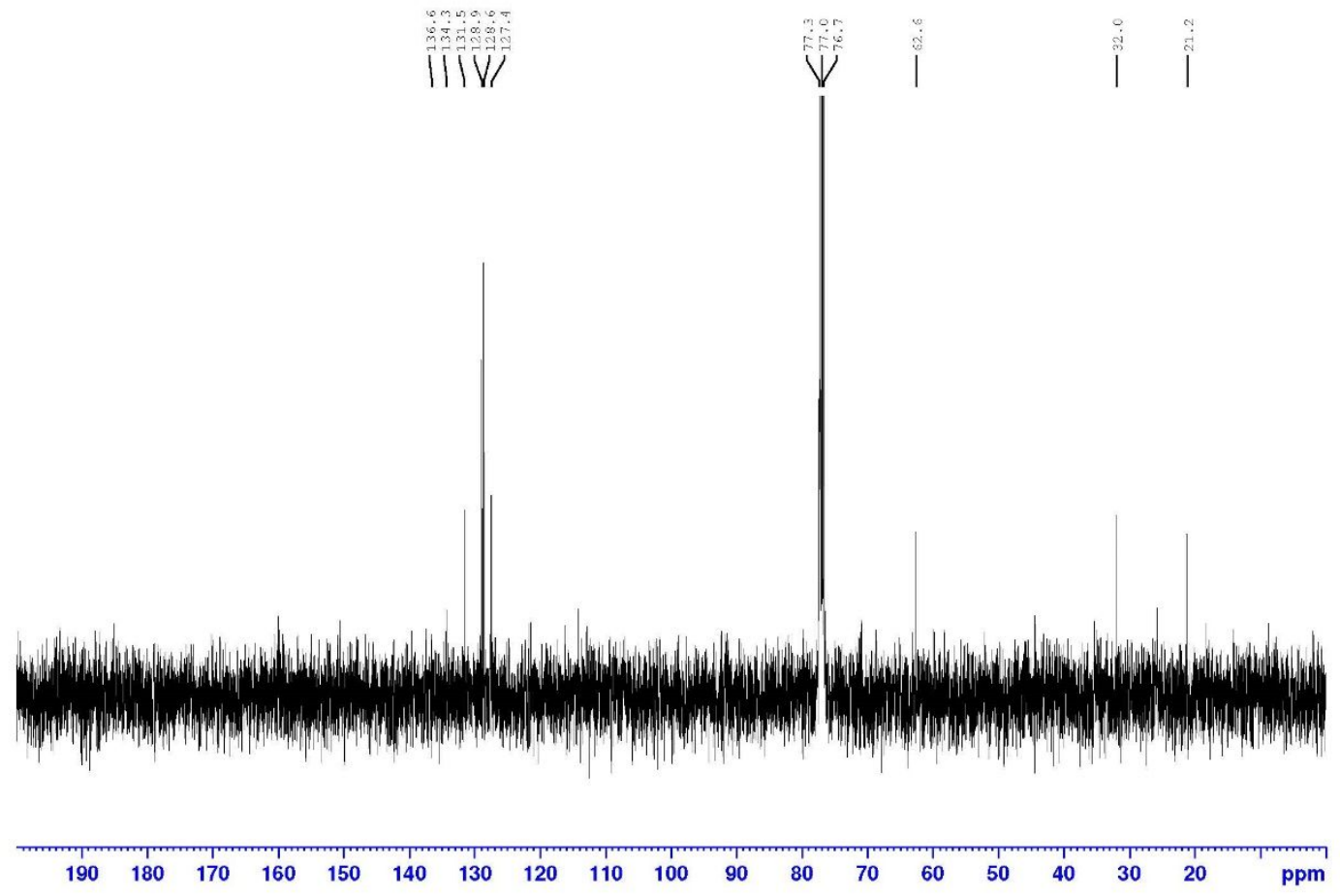

${ }^{1} \mathrm{H}$ NMR Spectrum of $(E)-3 \mathbf{w}, 400 \mathrm{MHz}, \mathrm{CDCl}_{3}$

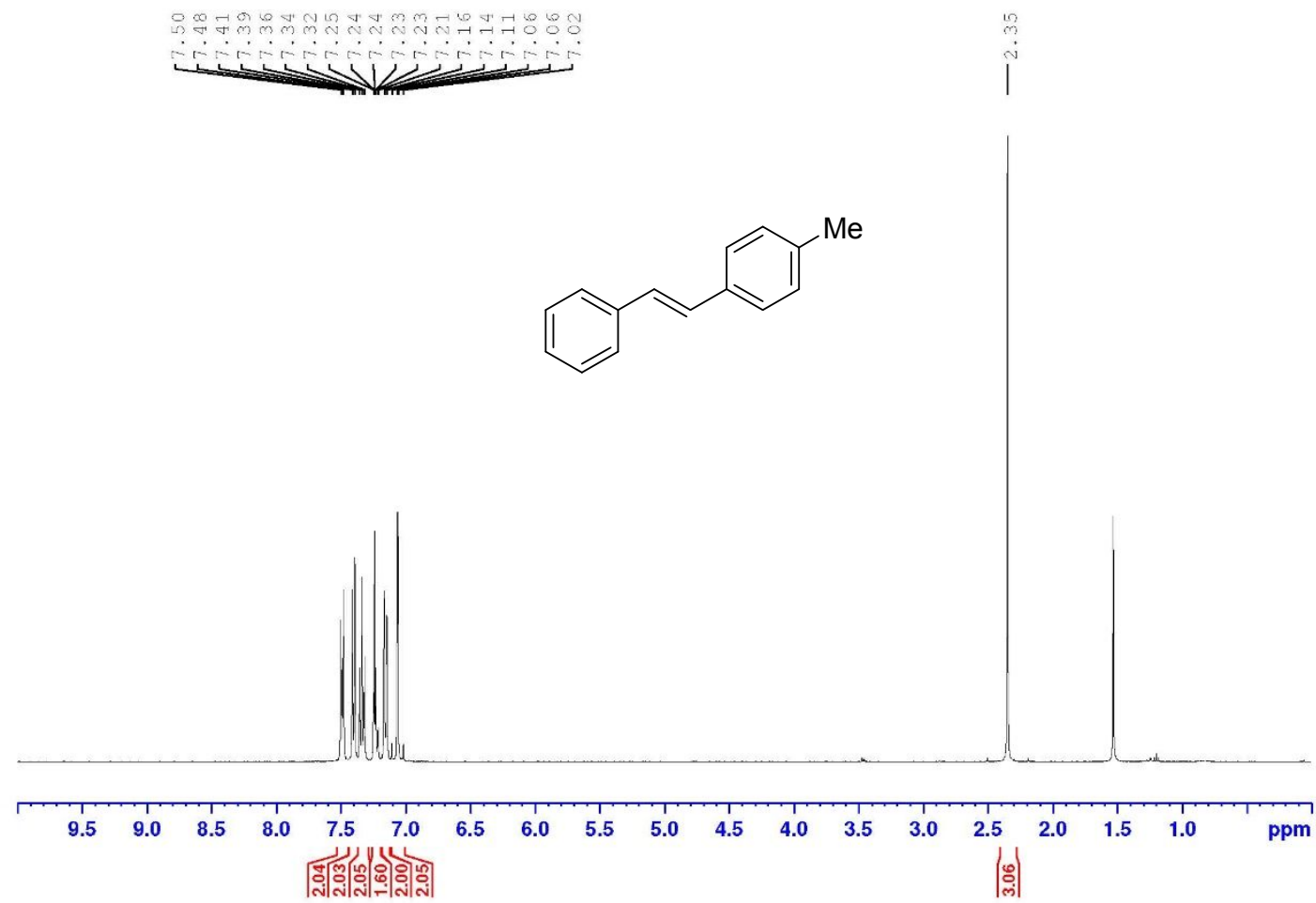

${ }^{13} \mathrm{C}$ NMR Spectrum of $(E)-3 w, 100 \mathrm{MHz}, \mathrm{CDCl}_{3}$ 


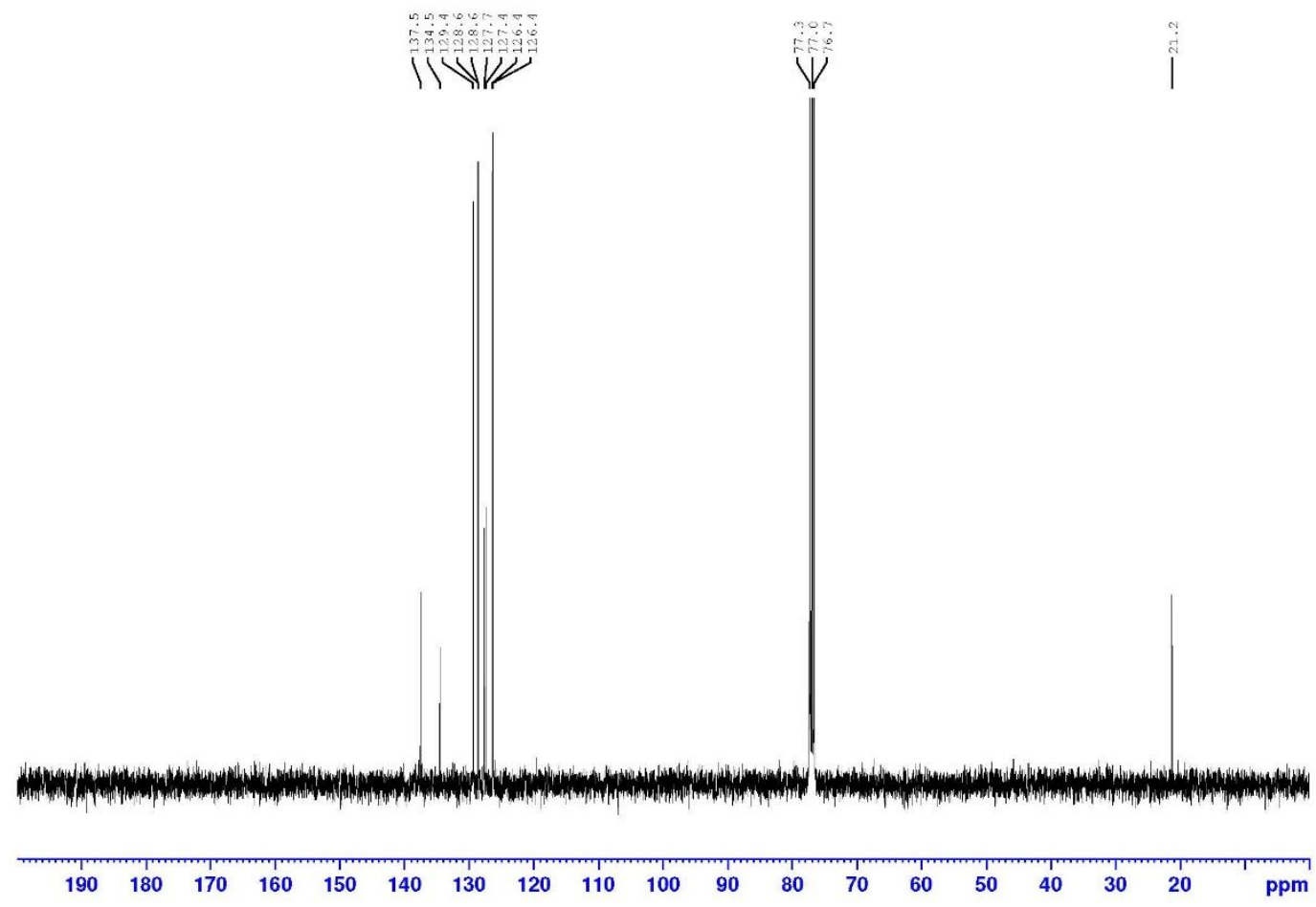

${ }^{1} \mathrm{H}$ NMR Spectrum of $(E)-3 \mathbf{x}, 400 \mathrm{MHz}, \mathrm{C}_{6} \mathrm{D}_{6}$

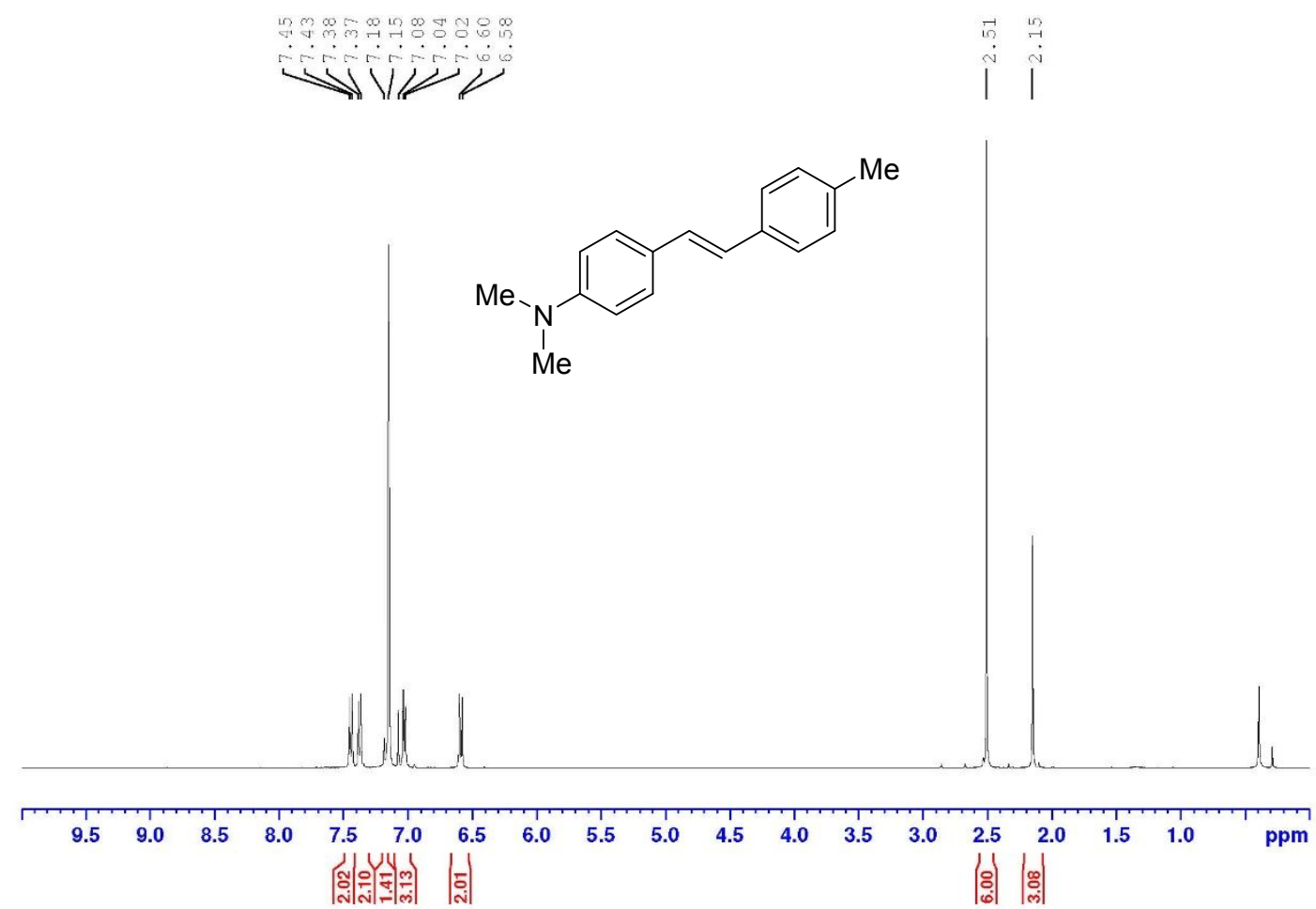

${ }^{13} \mathrm{C}$ NMR Spectrum of $(E)-3 \mathbf{x}, 100 \mathrm{MHz}, \mathrm{C}_{6} \mathrm{D}_{6}$ 


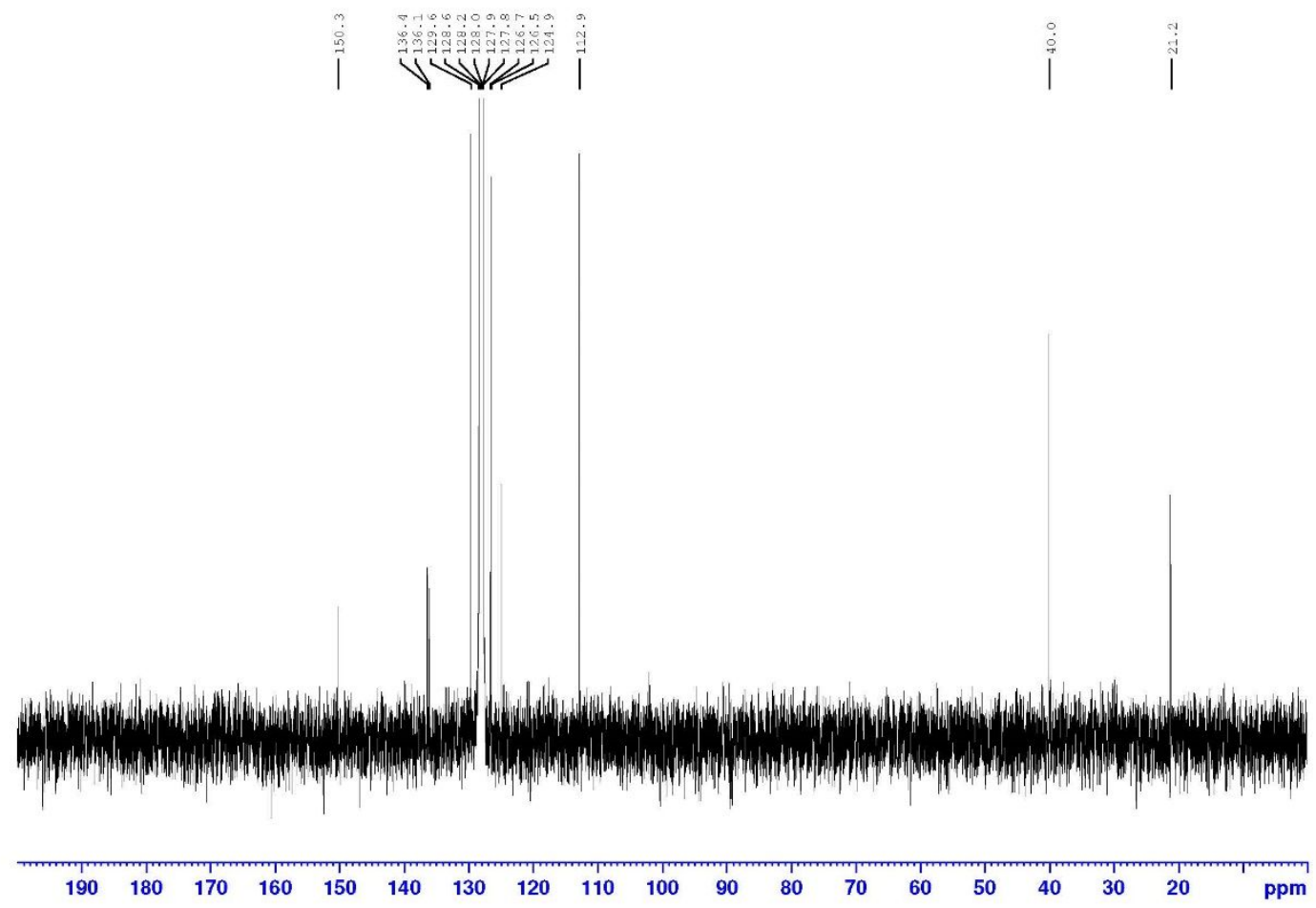

${ }^{1} \mathrm{H}$ NMR Spectrum of $(E)-3 y, 400 \mathrm{MHz}, \mathrm{CDCl}_{3}$
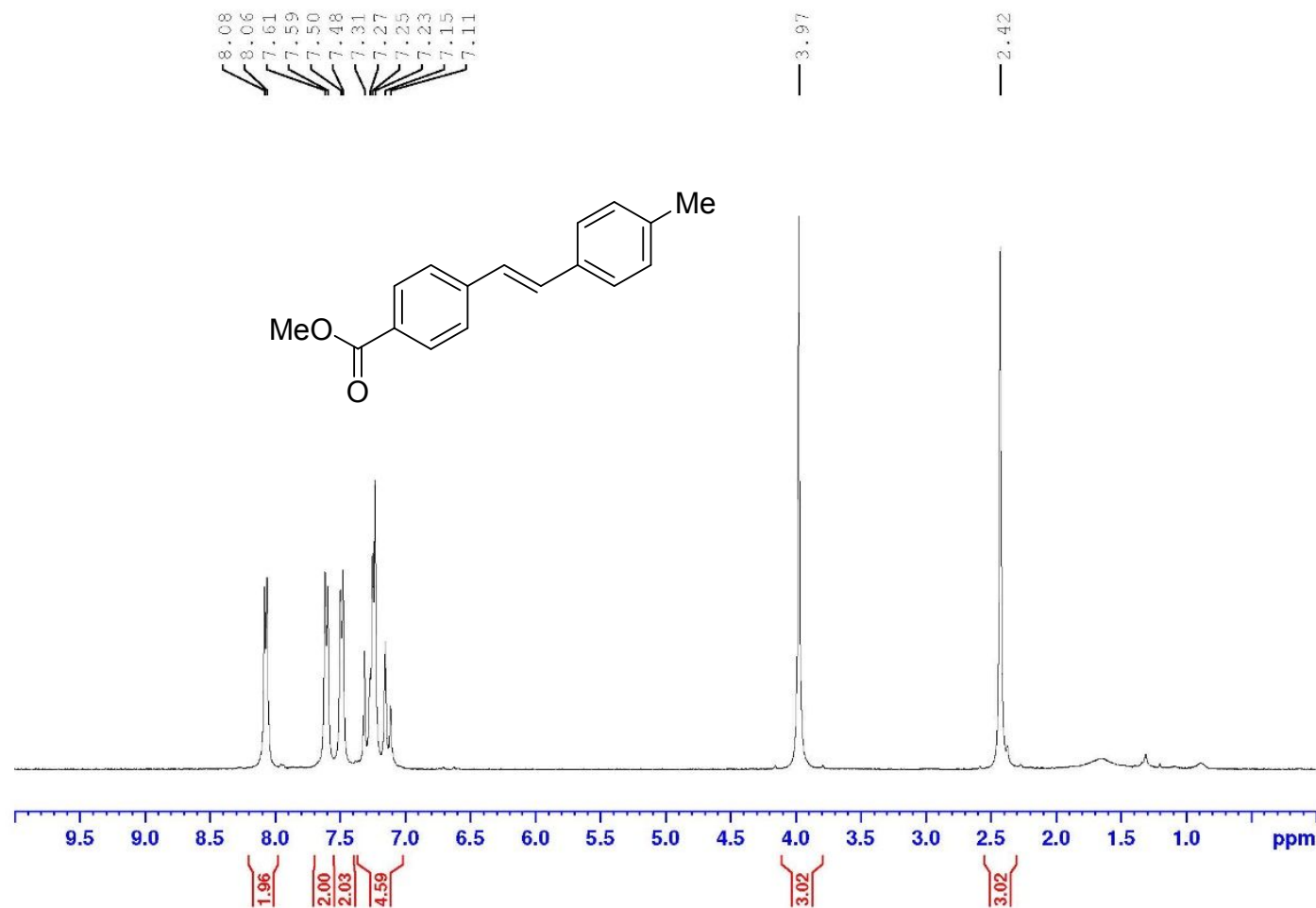

${ }^{13} \mathrm{C}$ NMR Spectrum of (E)-3y, $100 \mathrm{MHz}, \mathrm{CDCl}_{3}$ 


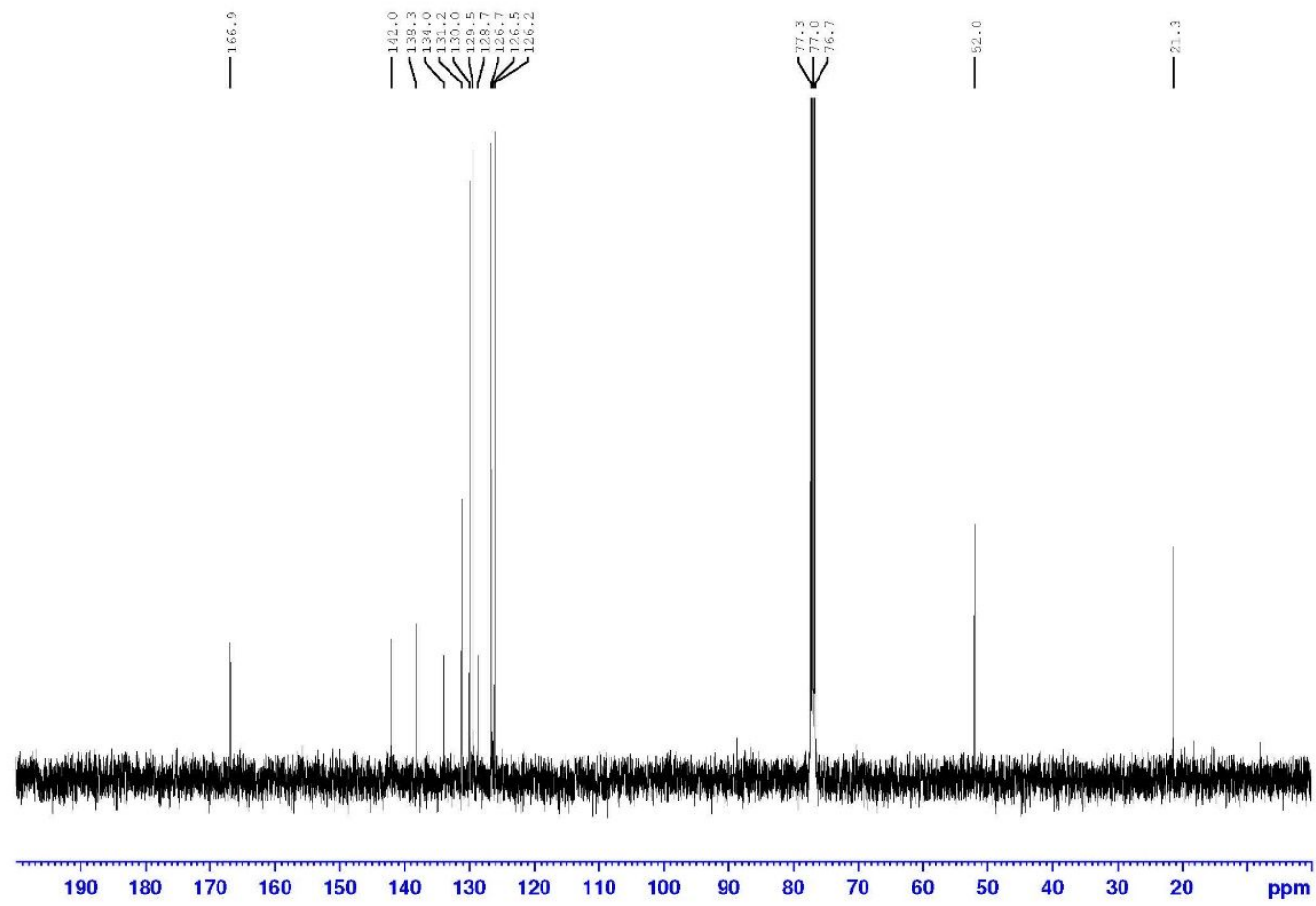

${ }^{1} \mathrm{H}$ NMR Spectrum of $(E)-3 z, 400 \mathrm{MHz}, \mathrm{CDCl}_{3}$

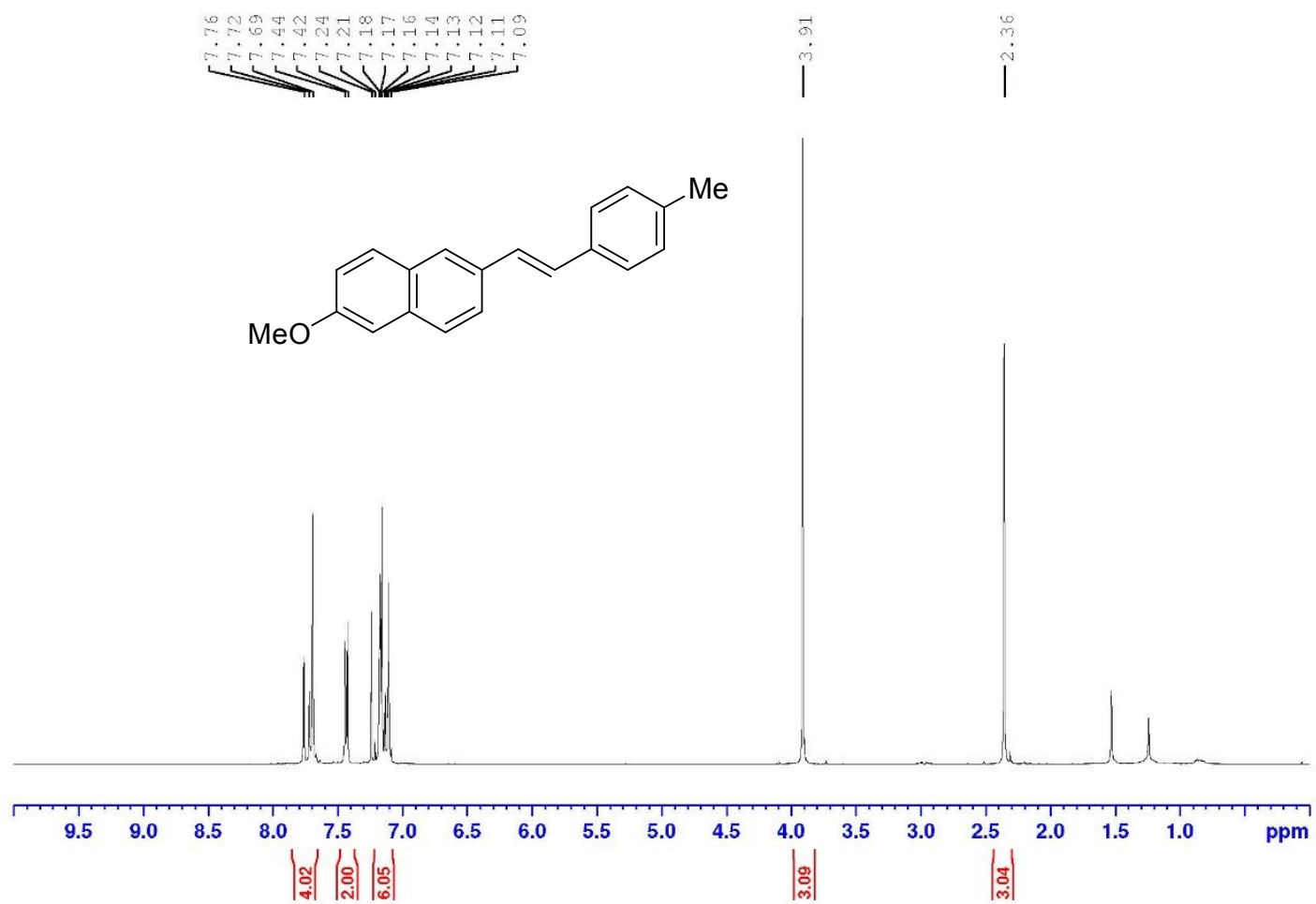

${ }^{13} \mathrm{C}$ NMR Spectrum of $(E)-3 z, 100 \mathrm{MHz}, \mathrm{CDCl}_{3}$ 

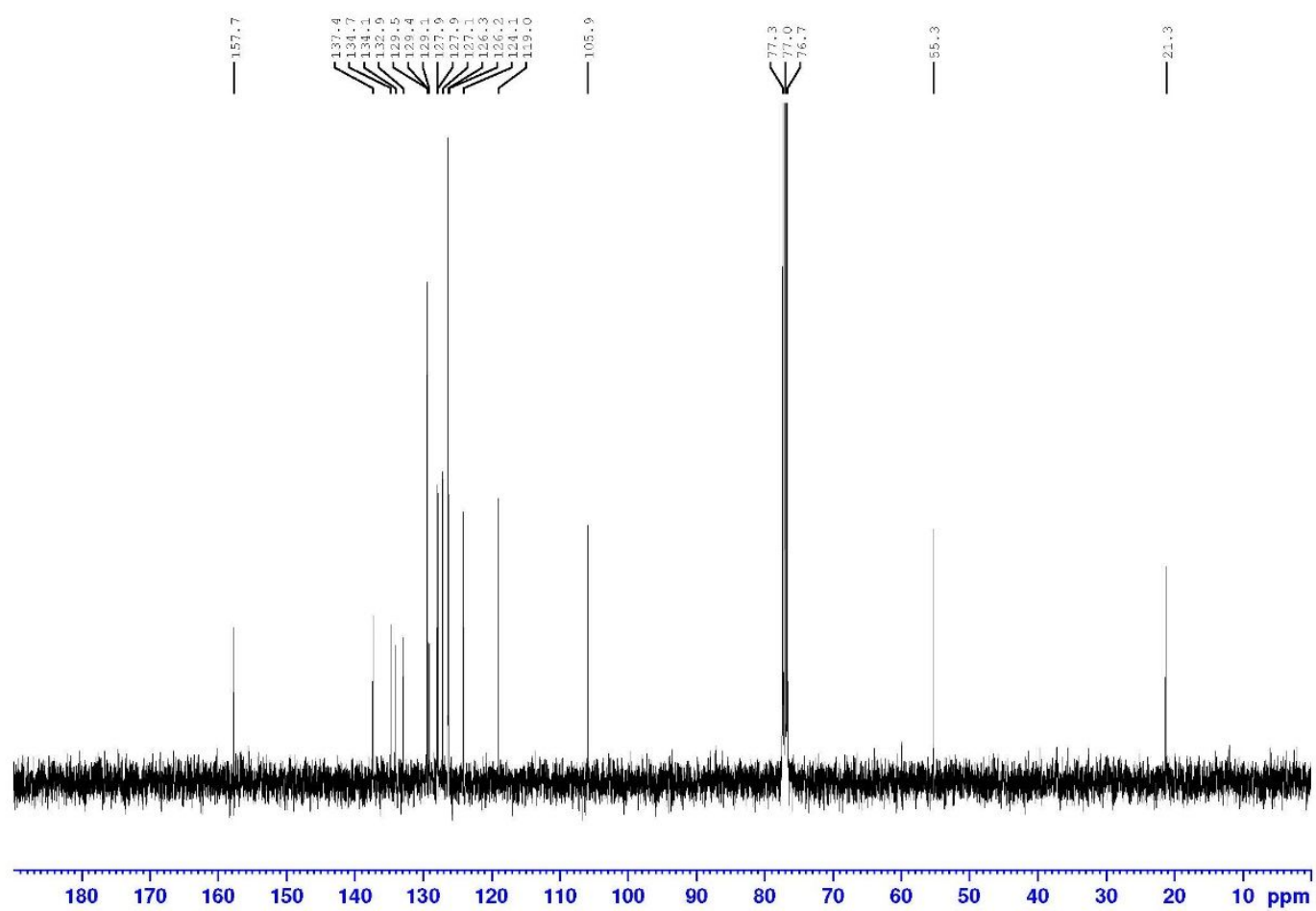

${ }^{1} \mathrm{H}$ NMR Spectrum of (E)-and (Z)-3aa, $400 \mathrm{MHz}, \mathrm{CDCl}_{3}$

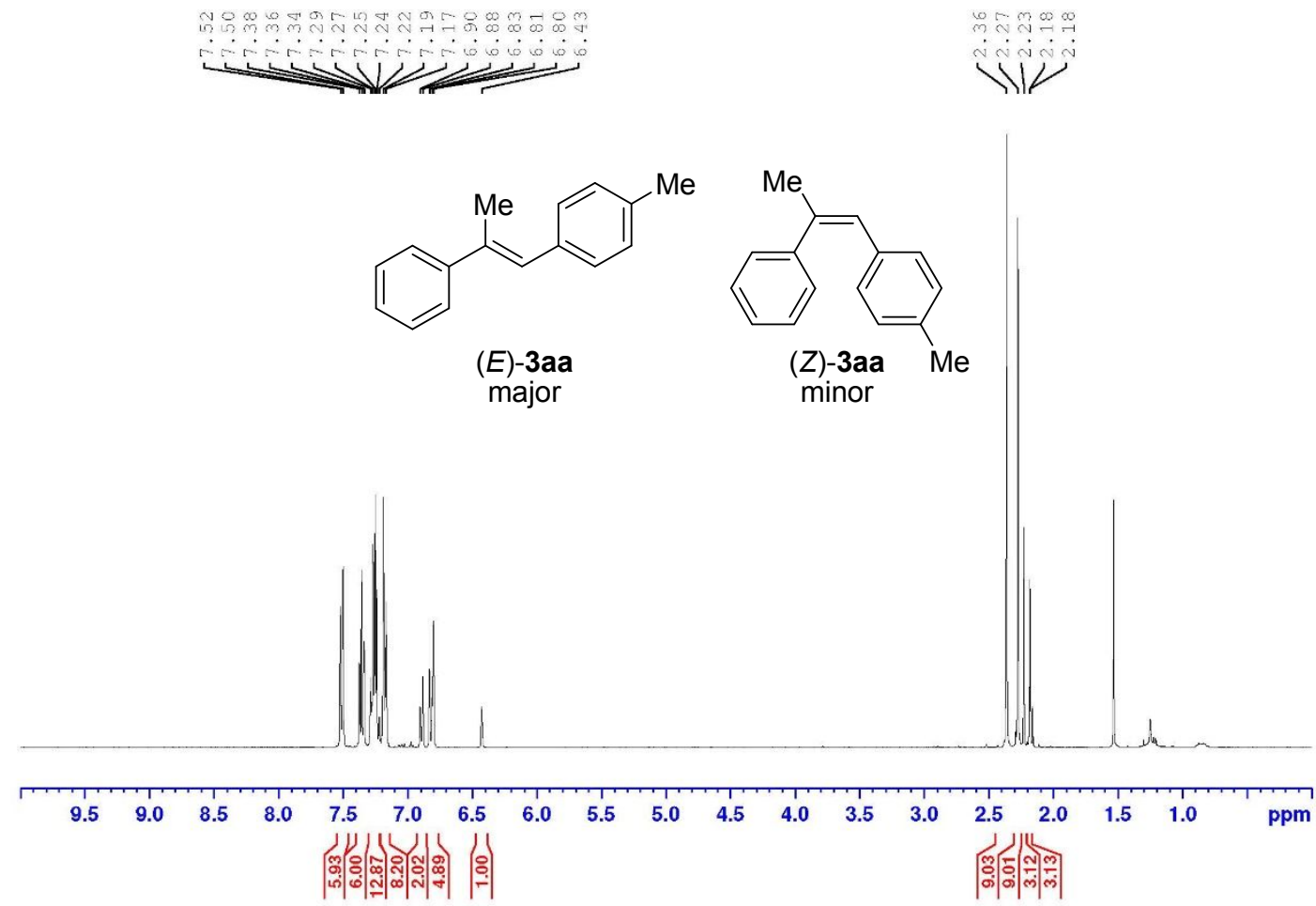

${ }^{13} \mathrm{C}$ NMR Spectrum of (E)-and (Z)-3aa, $100 \mathrm{MHz}^{\mathrm{CDCl}} \mathrm{CD}_{3}$ 


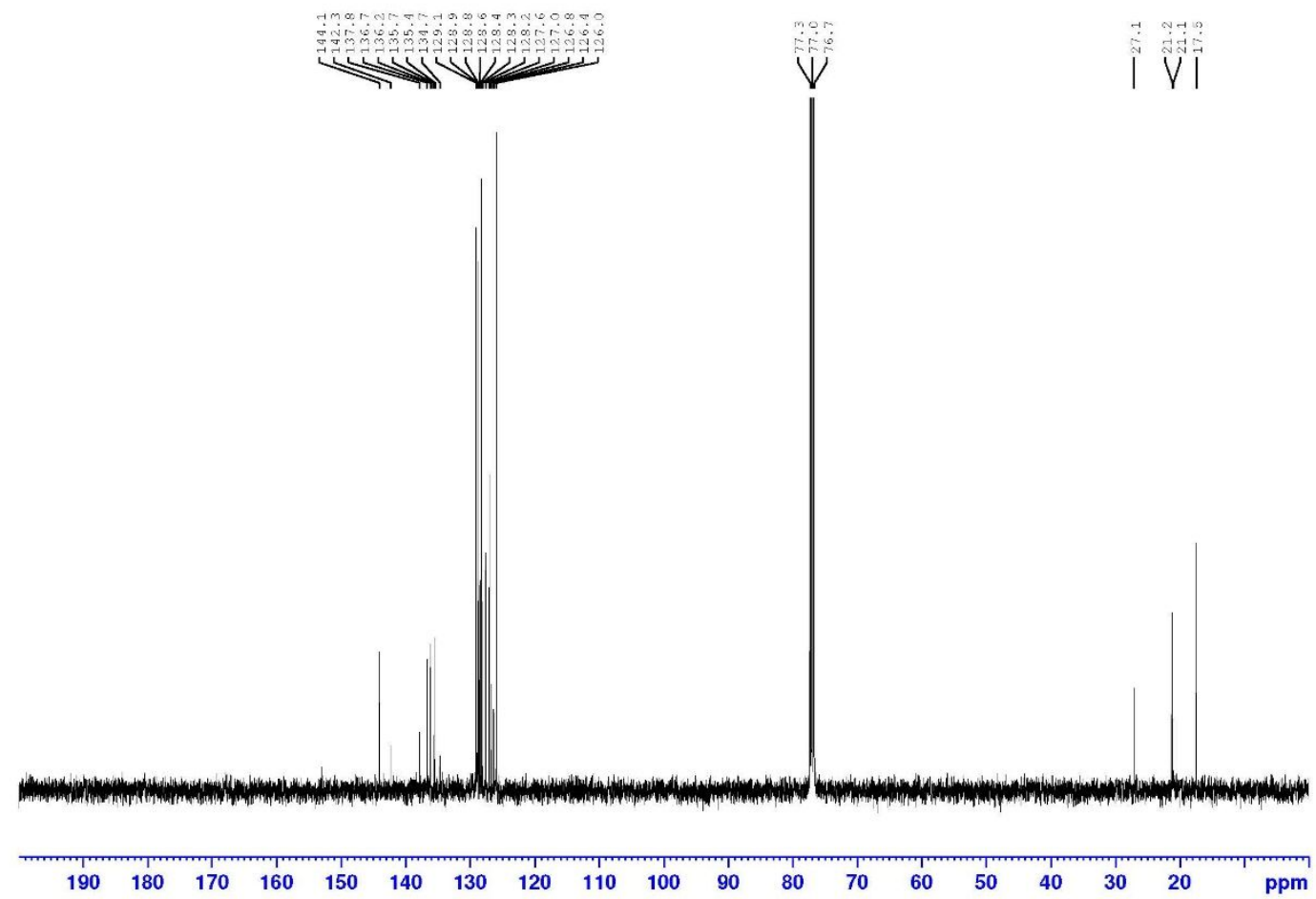

${ }^{1} \mathrm{H}$ NMR Spectrum of DMU-212, $400 \mathrm{MHz}, \mathrm{CDCl}_{3}$
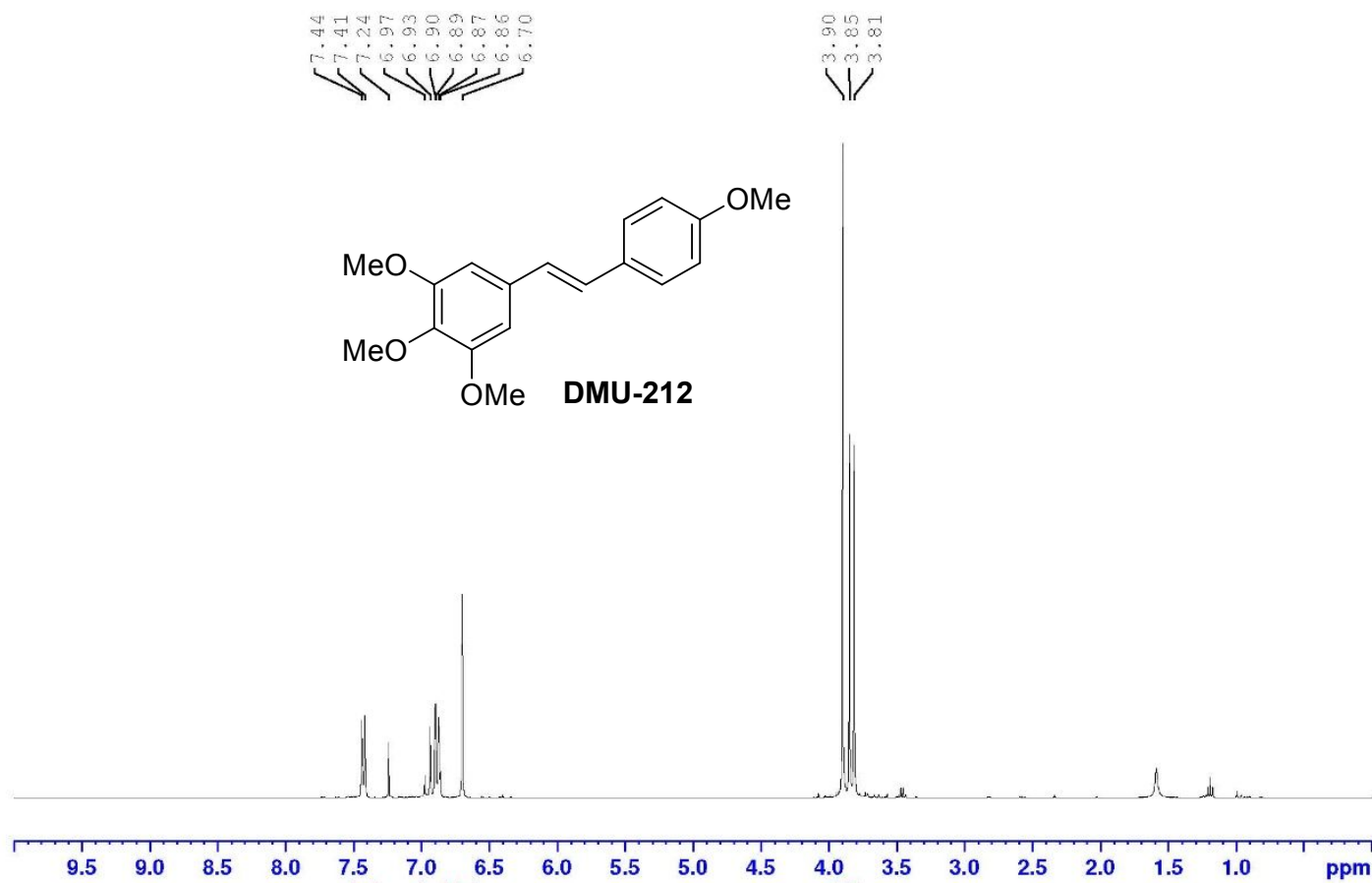

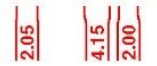

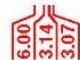

${ }^{13} \mathrm{C}$ NMR Spectrum of DMU-212, $100 \mathrm{MHz}, \mathrm{CDCl}_{3}$ 


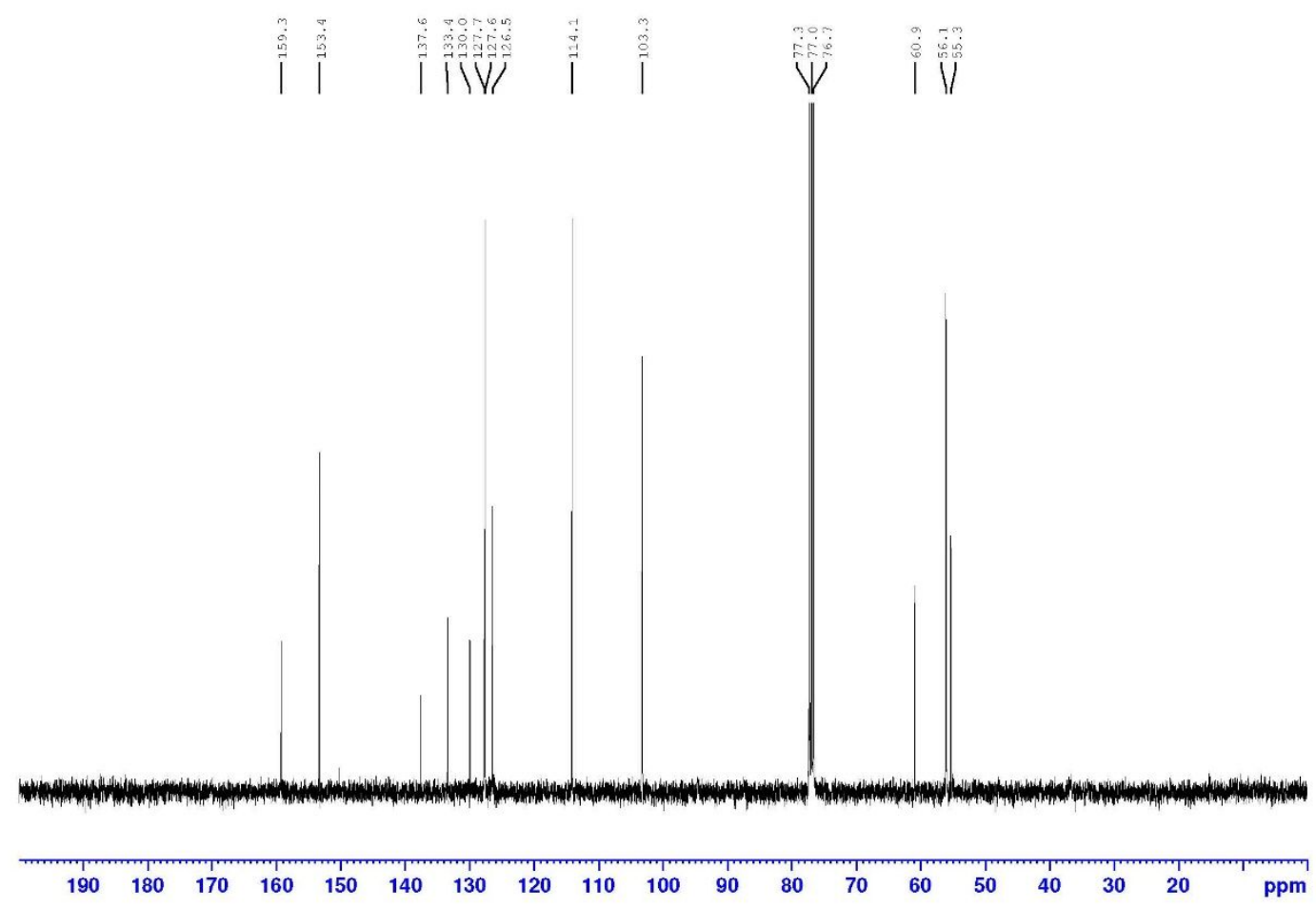

